\title{
Safeguards Technology for Thorium Fuel Cycles
}

\section{Research and Development Needs Assessment and Recommendations}

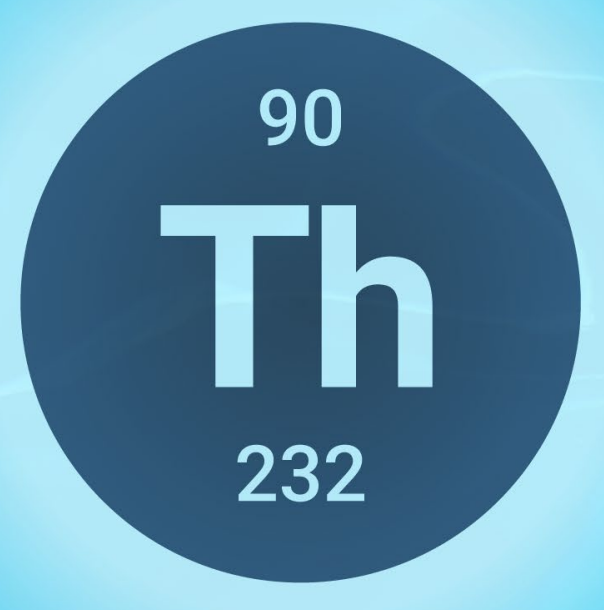

May 2021 


\title{
Safeguards Technology for Thorium Fuel Cycles: Research and Development Needs Assessment and Recommendations
}

Louise G. Evans, Vlad Henzl, Alicia Swift, Nicholas Luciano, Richard Reed, Eric Cervi, Jill Cooley, John Creasy, Bryn Davies, Joanna S. Denton, Andrea Favalli, Brandon Grogan, Alan Krichinsky, Karen Koop Hogue, Madeline L. Lockhart, David J. Mercer, Allyn Milojevich, Jacob Stinnett, Andrew Worrall, and Jinan Yang

Date Published: May 2021

\author{
Prepared by \\ OAK RIDGE NATIONAL LABORATORY \\ Oak Ridge, TN 37831-6283 \\ managed by \\ UT-BATTELLE, LLC \\ for the \\ US DEPARTMENT OF ENERGY \\ under contract DE-AC05-00OR22725
}




\section{Contents}

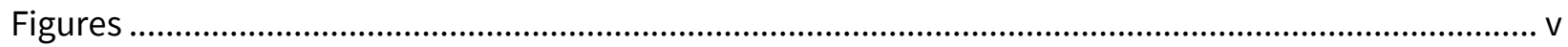

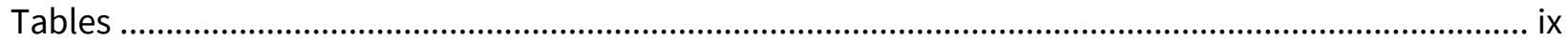

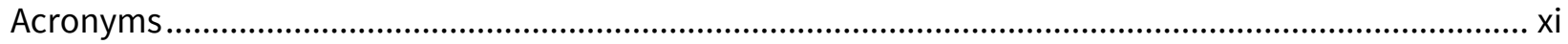

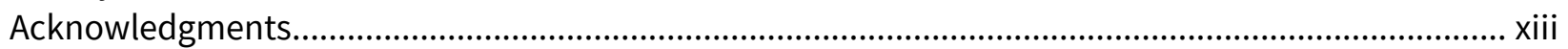

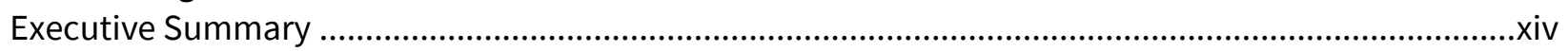

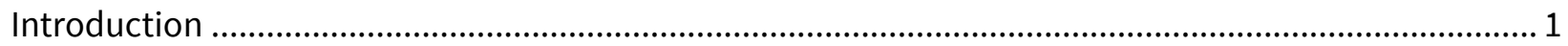

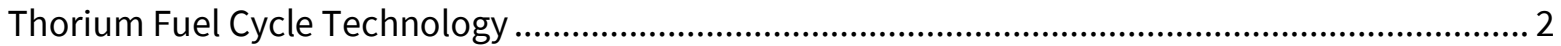

Mission Relevance: Proliferation Concerns and Challenges ….................................................... 3

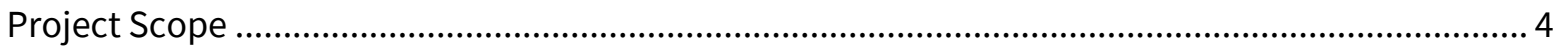

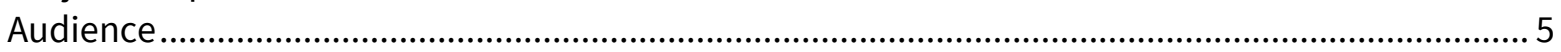

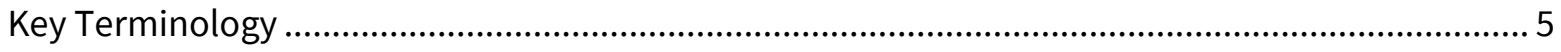

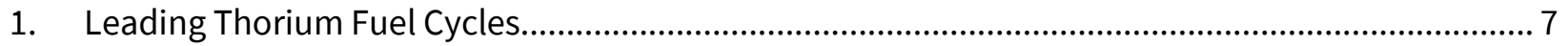

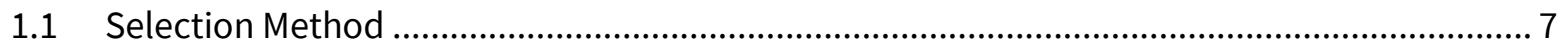

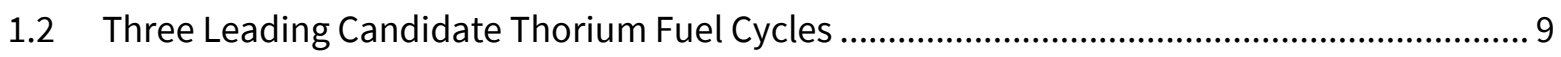

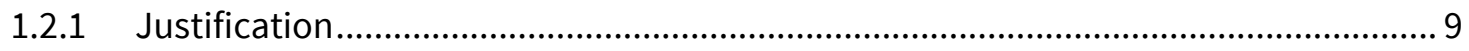

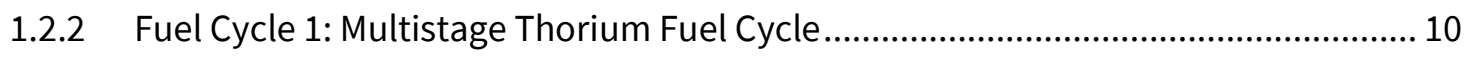

1.2.3 Fuel Cycle 2: Once-through Thorium Fuel Cycle in a PWR ....................................... 11

1.2.4 Fuel Cycle 3: Continuous-recycle Thorium Fuel Cycle in an MSR ............................ 12

1.3 Key Observations Affecting Implementation of Safeguards Technology ............................. 13

1.3.1 Varying Isotopic Mixtures, Fuel Forms, and Enrichment Levels............................... 13

1.3.2 Other Technical Factors Affecting Implementation of Safeguards Technology....... 14

1.3.3 Safeguards Technology R\&D Timeline: Safeguards Technology Development Timeline Compared with the Timeline to Commercial Implementation of

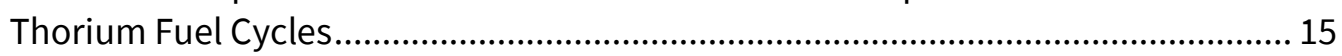

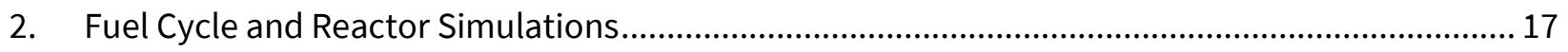

2.1 Objective of Fuel Cycle and Reactor Simulations ............................................................. 17

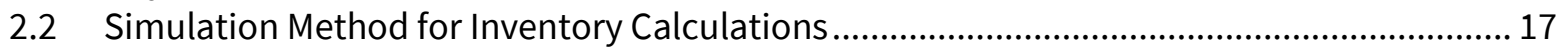

2.3 Simulated Thorium Fuel Cycle and Reactor Cases ........................................................... 18

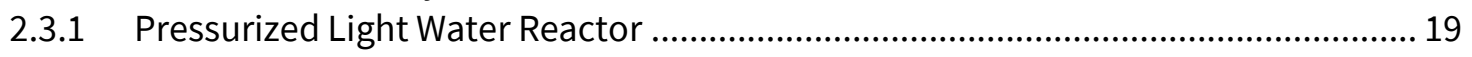

2.3.2 Pressurized Heavy Water Reactors ........................................................................ 33

2.3.3 Sodium-cooled Fast Breeder Reactor with a Thorium Breeding Blanket................. 41

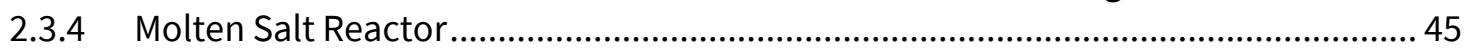

2.3.5 Comparison of ${ }^{233} \mathrm{U}$ and ${ }^{232} \mathrm{U}$ Inventories Across Reactor Types .............................. 47

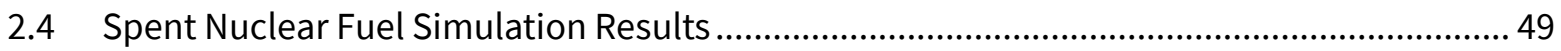

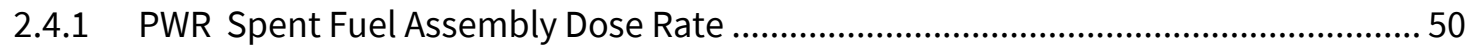

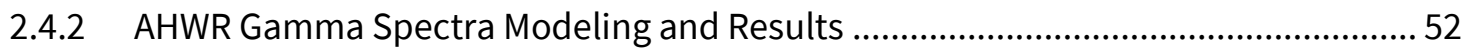

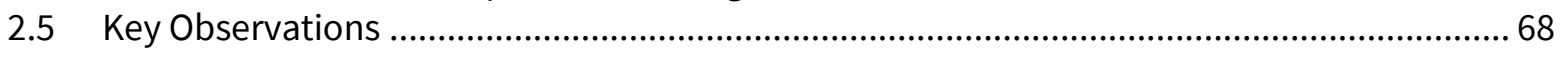

3. Assessment of Thorium Fuel Cycle Safeguards Relevant Radiation Signatures ............................69

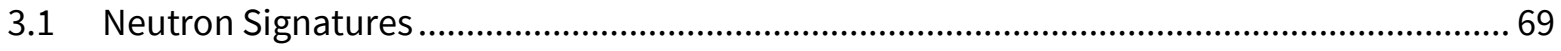

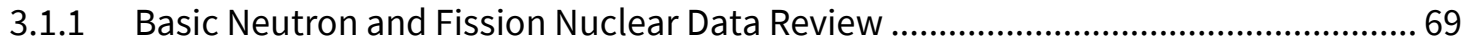

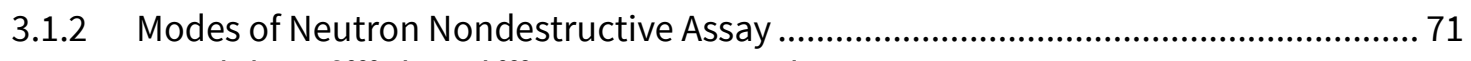

3.1.3 Feasibility of ${ }^{232} \mathrm{Th}$ and ${ }^{233} \mathrm{U}$ Neutron Nondestructive Assay................................... 77 
3.1.4 Overview of Simulated and Experimental Validation of Selected Neutron

Signature Properties

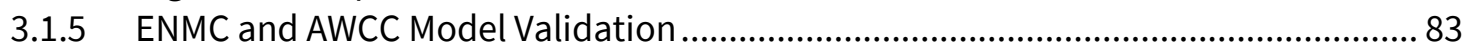

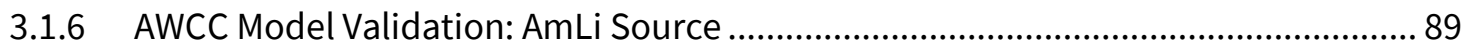

3.1.7 MCNP Simulations: Passive and Active Assay of Thorium-bearing Material............. 90

3.1.8 MCNP Simulations: Active Assay of Nixed ${ }^{233} \mathrm{U} / 232$ Th Samples in AWCC ................... 92

3.1.9 MCNP simulations: Passive and Active Assay of Mixed Pu/232Th Samples Representative of the Identified Thorium Fuel Compositions ................................. 93

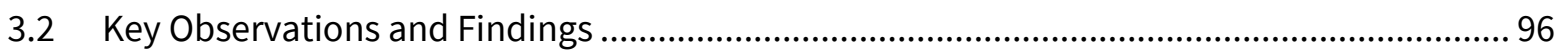

3.2.1 Conclusions from Neutron Measurement Simulations and Experiments ................96

3.2.2 Feasibility of Neutron NDA Techniques for ${ }^{233} \mathrm{U}$ - and Thorium-bearing Material ..... 97

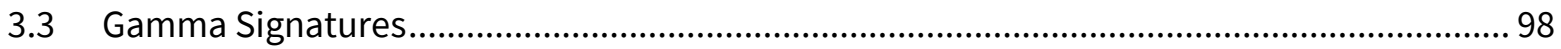

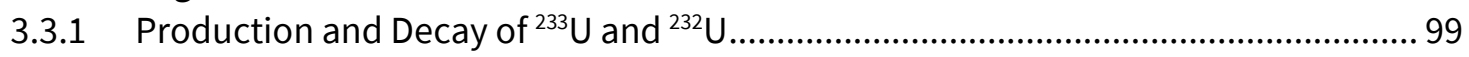

3.3.2 Dose Rates and Shielding Considerations Related to ${ }^{232} \mathrm{U}$ Contamination.............. 101

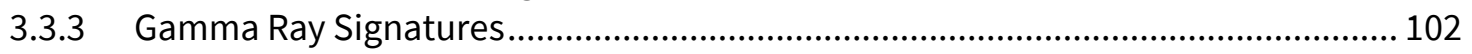

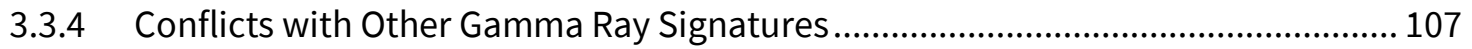

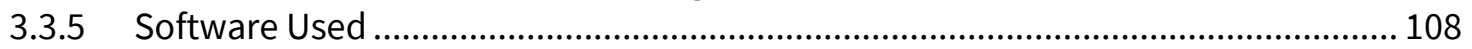

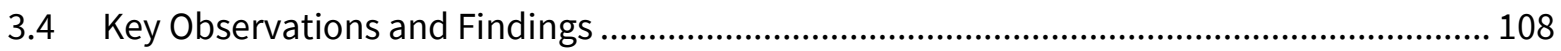

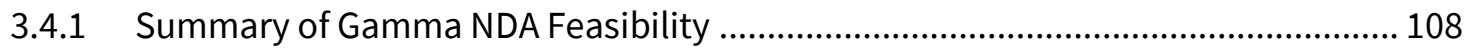

3.5 Destructive Assay in the Context of Thorium Fuel Cycles.................................................. 109

3.5.1 Overview of destructive assay techniques at the IAEA .......................................... 109

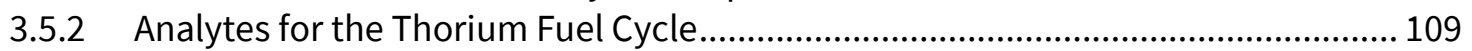

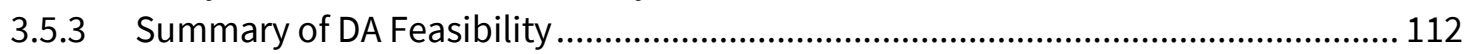

4. Overview of the Legal Framework for Implementation of International Nuclear Safeguards

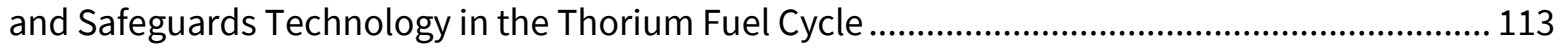

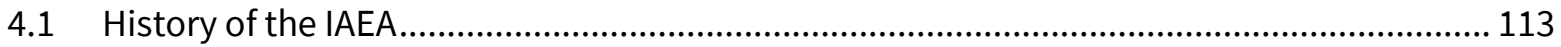

4.2 Legal Framework for International Nuclear Safeguards ................................................ 113

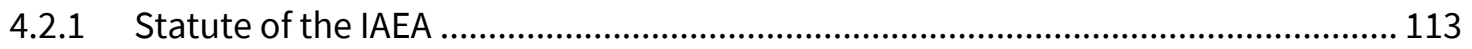

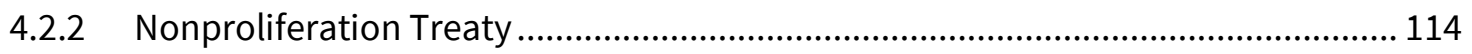

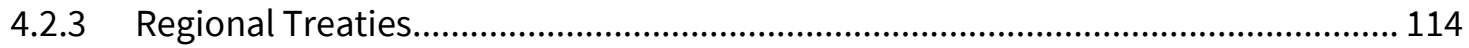

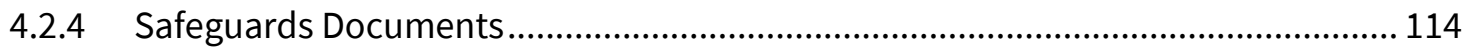

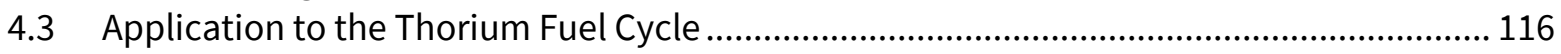

4.4 Complementary Access in the Context of Thorium Fuel Cycles ........................................ 117

4.4.1 Overview of Complementary Access under the Additional Protocol ....................... 117

4.4.2 Complementary Access Toolkit ............................................................................. 118

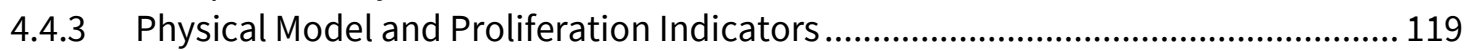

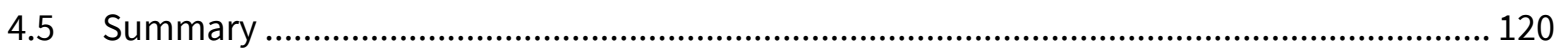

5. R\&D Needs for a Thorium Fuel Cycle Safeguards Technology Development Effort...................... 121

5.1 R\&D Needs-Lower TRL (1-4) Concept Development and Laboratory Demonstration ...... 122

5.2 R\&D Needs-Higher TRL (5+) Technologies and Application-Specific Demonstration,

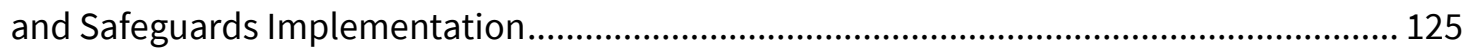

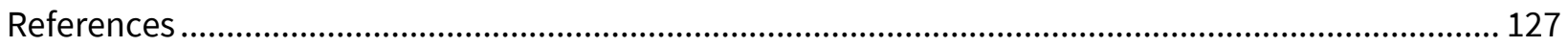

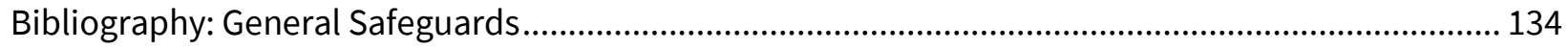

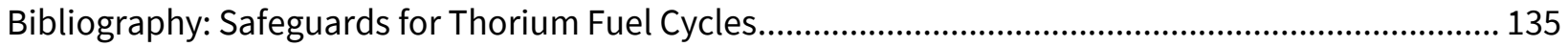




\section{Figures}

Figure 1. Multistage thorium fuel cycle.

Figure 2. Once-through thorium fuel cycle in a PWR.

Figure 3. Continuous-recycle thorium fuel cycle in an MSR. ......................................................... 13

Figure 4. Sample timeline to commercial implementation of a leading thorium fuel cycle................... 15

Figure 5. Example thorium fuel cycle implementation timeline (top) vs. example safeguards

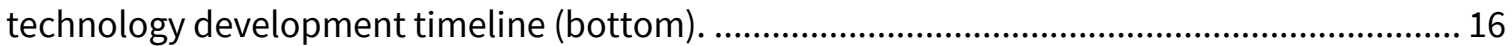

Figure 6. Homogeneous PLWR quarter fuel lattice.

Figure 7. Th, U, Pu, mass concentrations vs. EFPDs for the homogeneous PLWR fuel lattice composed of $100 \% \mathrm{UO}_{2}$ enriched from 1 to $20 \%$.

Figure 8. Homogeneous PLWR quarter fuel lattice.

Figure 9. Th, $\mathrm{U}$, and Pu, mass concentrations vs. EFPDs for the homogeneous PLWR fuel lattice composed of $5 \% \mathrm{Th} / 95 \% \mathrm{U}$ enriched from 1 to $20 \%$ enrichment.

Figure 10. Th, $\mathrm{U}$, and $\mathrm{Pu}$, mass concentrations vs. EFPDs for the homogeneous PLWR fuel lattice composed of $95 \% \mathrm{Th} / 5 \% \mathrm{U}$ enriched from 1 to $20 \%$ enrichment.

Figure 11. Heterogeneous PLWR quarter fuel lattice.

Figure 12. Th, $\mathrm{U}$, and $\mathrm{Pu}$, mass concentrations vs. EFPDs for the heterogeneous PLWR fuel lattice blanket composed of $85 \% \mathrm{Th} / 15 \% \mathrm{U}$ at a range of enriched from $1 \%$ to $5 \%$ enrichment, with a $5 \%$ enriched $\mathrm{UO}_{2}$ seed region.

Figure 13. Th, $\mathrm{U}$, and $\mathrm{Pu}$, mass concentrations vs. EFPDs for the heterogeneous PLWR fuel lattice blanket composed of $10 \% \mathrm{Th} / 90 \% \mathrm{U}$ at a range of enriched from $1 \%$ to $5 \%$ enrichment, with a $5 \%$ enriched $\mathrm{UO}_{2}$ seed region.

Figure 14. Th, $\mathrm{U}$, and $\mathrm{Pu}$, mass concentrations vs. EFPDs for the heterogeneous PLWR fuel lattice blanket composed of $85 \% \mathrm{Th} / 15 \% \mathrm{U}$ at a range of enriched from $1 \%$ to $5 \%$ enrichment, with a $1 \%$ enriched $\mathrm{UO}_{2}$ seed region.

Figure 15. Th, $\mathrm{U}$, and Pu, mass concentrations vs. EFPDs for the heterogeneous PLWR fuel lattice blanket composed of $10 \% \mathrm{Th} / 90 \% \mathrm{U}$ enriched from $1 \%$ to $5 \%$, with a $1 \%$ enriched $\mathrm{UO}_{2}$ seed region.

Figure 16. Th, $\mathrm{U}$, and $\mathrm{Pu}$, mass concentrations vs. EFPDs for the homogeneous PLWR fuel lattice with ${ }^{233} \mathrm{U}$ makeup for recycle Generations $1-5$.

Figure 17. Th, $\mathrm{U}$, and Pu, mass concentrations vs. EFPDs for the heterogeneous PWR fuel lattice with ${ }^{233} \mathrm{U}$ makeup and constant seed composition (4 $\mathrm{wt} \%$ enriched $\mathrm{UO}_{2}$ ) for recycle Generations 1-5.

Figure 18. Th, $\mathrm{U}$, and $\mathrm{Pu}$, mass concentrations vs. EFPDs for the heterogeneous PLWR fuel lattice with ${ }^{233} \mathrm{U}$ makeup and constant blanket composition ( $\left.70 \% \mathrm{Th} / 30 \% \mathrm{U}\right)$ for recycle Generations 1-5....

Figure 19. Th, $\mathrm{U}$, and $\mathrm{Pu}$, mass concentrations vs. EFPDs for the homogeneous PHWR fuel lattice with ${ }^{235} \mathrm{U}$ makeup for recycle Generations $1-5$.

Figure 20. Th, U, and Pu, mass concentrations vs. EFPDs for the heterogeneous PHWR fuel lattice with ${ }^{235} \mathrm{U}$ makeup and constant seed composition ( $4 \mathrm{wt} \%$ enriched $\mathrm{UO}_{2}$ ) for recycle Generations 1-5.

Figure 21. Th, $\mathrm{U}$, and $\mathrm{Pu}$, mass concentrations vs. EFPDs for the heterogeneous PHWR fuel lattice with ${ }^{235} \mathrm{U}$ makeup and constant blanket composition ( $\left.70 \% \mathrm{Th} / 30 \% \mathrm{U}\right)$ for recycle Generations $1-5$. 
Figure 22. Natural uranium $\mathrm{UO}_{2}$ 28-element CANDU fuel lattice

Figure 23. Th, $\mathrm{U}$, and $\mathrm{Pu}$, mass concentrations vs. EFPDs for the natural uranium $\mathrm{UO}_{2} 28$ element CANDU fuel lattice.

Figure 24. AHWR fuel lattice.

Figure 25. Th, $\mathrm{U}$, and $\mathrm{Pu}$, mass concentrations vs. EFPDs for the AHWR Initial Type I fuel cluster showing composition of the inner, middle, and outer rings.

Figure 26. Th, U, and Pu, mass concentrations vs. EFPDs for the AHWR Initial Type II fuel cluster showing composition of the inner, middle, and outer rings.

Figure 27. Th, $\mathrm{U}$, and Pu, mass concentrations vs. EFPDs for the AHWR Transition fuel cluster showing composition of the inner, middle, and outer rings.

Figure 28. Th, $\mathrm{U}$, and $\mathrm{Pu}$, mass concentrations vs. EFPDs for the AHWR Equilibrium fuel cluster showing composition of the inner, middle, and outer rings.

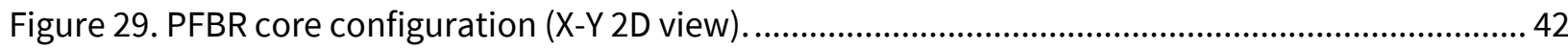

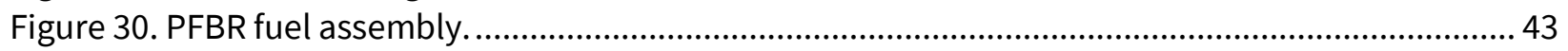

Figure 31. Th, $\mathrm{U}$, and $\mathrm{Pu}$, mass concentrations vs. EFPDs for the PFBR driver fuel. ............................. 44

Figure 32. Th, $\mathrm{U}$, and $\mathrm{Pu}$, mass concentrations vs. EFPDs for the PFBR axial blanket............................ 44

Figure 33. Th, $\mathrm{U}$, and $\mathrm{Pu}$, mass concentrations vs. EFPDs for the PFBR radial blanket......................... 45

Figure 34. Core design of the MSBR showing the multiple zones optimized for power

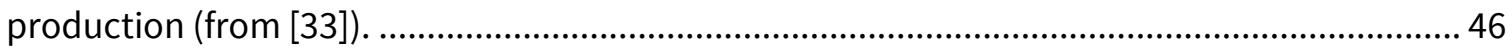

Figure 35. Th, U, Pu, mass concentrations vs. EFPDs for the MSBR fuel salt. ........................................ 46

Figure 36. Comparison across reactor types of the mass concentration of ${ }^{233} \mathrm{U}$ per initial thorium.

Figure 37. Comparison across reactor types of the mass concentration of ${ }^{233} \mathrm{U}$ per initial thorium.

Figure 38. 3D PWR assembly with dose-rate detector at $1 \mathrm{~m}$ from the axial middle of the assembly....

Figure 39. (Top) The absolute gamma dose rates from a bare $\mathrm{ThO}_{2}-\mathrm{UO}_{2} \mathrm{PWR}$ assembly and an equivalent $\mathrm{UO}_{2}$ assembly.

Figure 40. Simulated spectra of the five assembly types at 30 days after discharge from the reactor.

Figure 41. Close-up of the simulated spectra at 30 days of cooling time showing the $0-500 \mathrm{keV}$ energy range... 55

Figure 42. The simulated spectra of the two nonthorium assemblies at 30 days of cooling time.......... 55

Figure 43 . The $500-1400 \mathrm{keV}$ region of the simulated spectra for the two nonthorium assemblies at 30 days of cooling time.

Figure 44. Simulated spectra of the three thorium-MOX assemblies at 30 days of cooling time. .......... 57

Figure 45 . The $500-1600 \mathrm{keV}$ region of the simulated spectra of the three thorium-MOX assemblies at 30 days of cooling time.

Figure 46. Simulated spectra of the five assembly types at 1 year after discharge from the reactor.

Figure 47. The simulated spectra of the two nonthorium assemblies at 1 year of cooling time.

Figure 48. The $800-1600 \mathrm{keV}$ region of the simulated spectra for the two nonthorium assemblies at 1 year of cooling time.

Figure 49. Simulated spectra of the three thorium-MOX assemblies at 1 year of cooling time.

Figure 50 . The $800-1250 \mathrm{keV}$ region of the simulated spectra of the three thorium-MOX assemblies at 1 year of cooling time. 
Figure 51. Simulated spectra of the five assembly types at 5 years after discharge from the

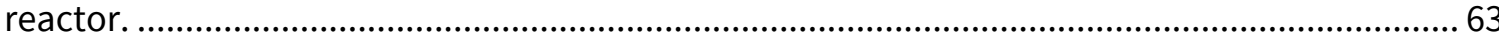

Figure 52. The simulated spectra of the two nonthorium assemblies at 5 years of cooling time. ......... 63

Figure 53. The 1300-1700 keV region of the simulated spectra for the two nonthorium

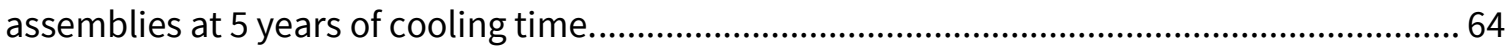

Figure 54. Simulated spectra of the three thorium-MOX assemblies at 5 years of cooling time........... 64

Figure 55. The 1000-1250 keV region of the simulated spectra of the three thorium-MOX assemblies at 5 years of cooling time.

Figure 56. Simulated spectra of the five assembly types at 20 years after discharge from the reactor.

Figure 57 . The simulated spectra of the two nonthorium assemblies at 20 years of cooling time.

Figure 58. Simulated spectra of the three thorium-MOX assemblies at 20 years of cooling time. .........67

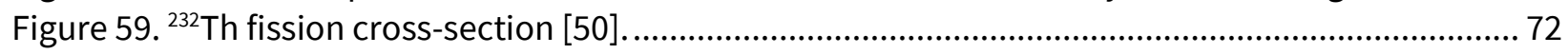

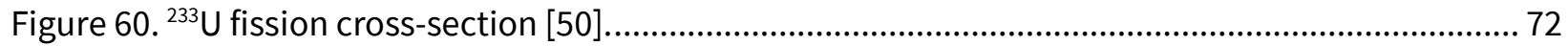

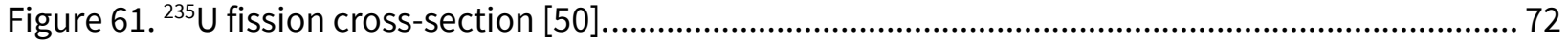

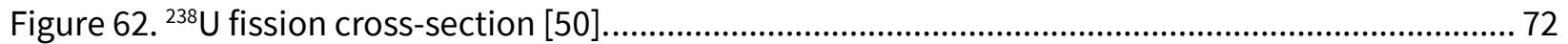

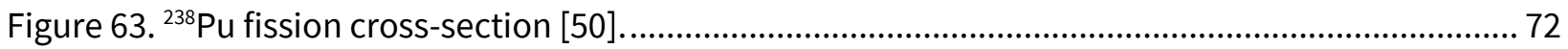

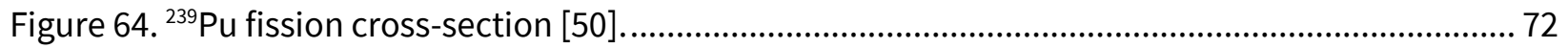

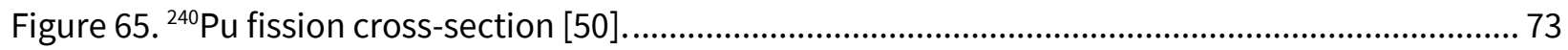

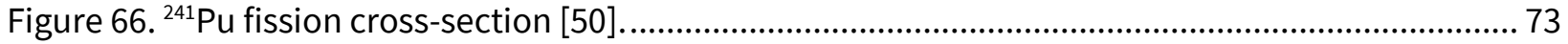

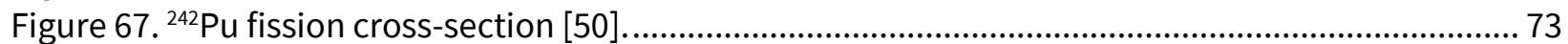

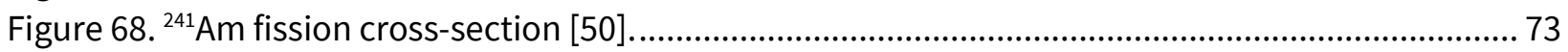

Figure 69. D-T neutron generator available from Thermo Scientific (model P385), including the electronics enclosure, emergency shutdown button module, accelerator tube, and indicator lamp [58].....

Figure 70. Energy spectrum of neutrons created in various isotopic sources by $(\alpha, n)$ reactions or spontaneous fission (simulations using GADRAS code [47]) to show the dynamic neutron energy range of available interrogation sources........................................................ 76

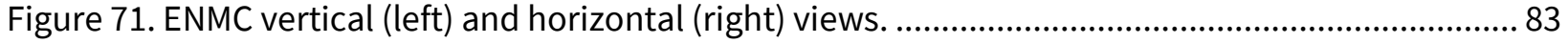

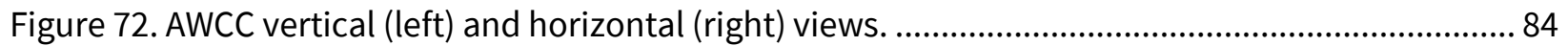

Figure 73. AWCC model with the AmLi source (green)................................................................... 91

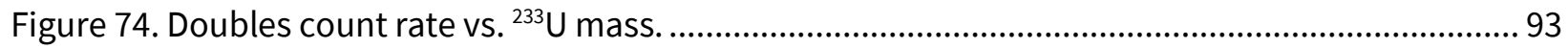

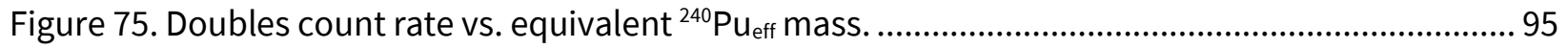

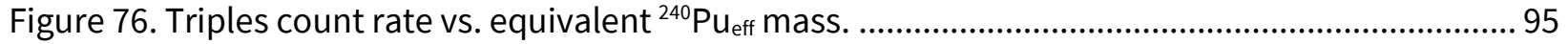

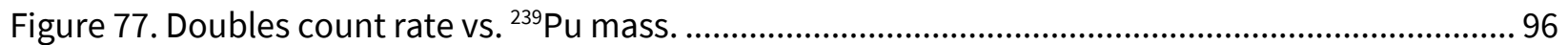

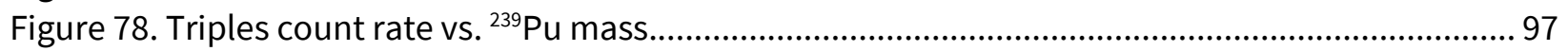

Figure 79. Production of ${ }^{233} \mathrm{U}$ and ${ }^{232} \mathrm{U}$ in a thorium fuel-based nuclear reactor. ................................ 100

Figure 80. Dose rate from a 1 gram ${ }^{233} \mathrm{U}$ item with $10 \mathrm{ppm}{ }^{232} \mathrm{U}$, ignoring self-attenuation.................. 100

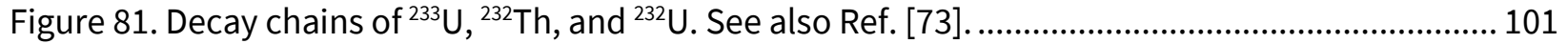

Figure 82. Annotated decay chains of ${ }^{232} \mathrm{Th}$ and ${ }^{232} \mathrm{U}$. Image modified from Ref. [75]......................... 102

Figure 83. Comparisons of a $1 \mathrm{~kg} \mathrm{ThO}_{2}$ spectrum (blue) with a $10 \mathrm{~g}^{233} \mathrm{U}$ metal with $10 \mathrm{ppm}{ }^{232} \mathrm{U}$ aged for 6 months spectrum (black), normalized by the $2614 \mathrm{keV}$ peak area.......................... 103

Figure 84. Simulated spectra of a $1 \mathrm{~g}^{233} \mathrm{U}$ source (10 ppm ${ }^{233} \mathrm{U}, 6$ months old) (black), measured with an identiFINDER-NG at $100 \mathrm{~cm}$ and natural background (blue). 
Figure 85. Simulated high-resolution spectra of a $1 \mathrm{~kg}{ }^{233} \mathrm{U}$ item with $10 \mathrm{ppm}{ }^{232} \mathrm{U}$ contamination (aged for 1 year) with $1 \mathrm{~cm}$ of lead shielding (blue) and without the shielding (black).

Figure 86. Simulated high-resolution spectra of a $1 \mathrm{~kg}^{233} \mathrm{U}$ item ( $10 \mathrm{ppm}{ }^{233} \mathrm{U}, 1$ year old) with 1 $\mathrm{cm}$ of lead shielding (blue) and without the shielding (black)

Figure 87. Simulated high-resolution spectra of a $1 \mathrm{~kg}{ }^{233} \mathrm{U}$ item ( $10 \mathrm{ppm}{ }^{232} \mathrm{U}, 1$ year old) with 1

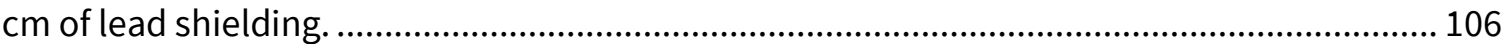

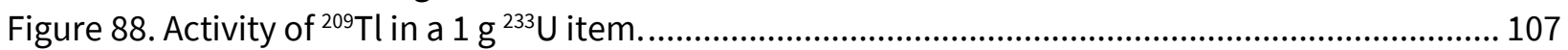

Figure 89. A measured spectrum of an $\sim 150$ gram ${ }^{233} \mathrm{U}$ source with only a few $p \mathrm{~mm}^{232} \mathrm{U}$

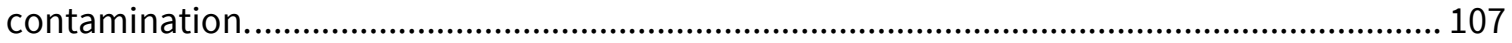

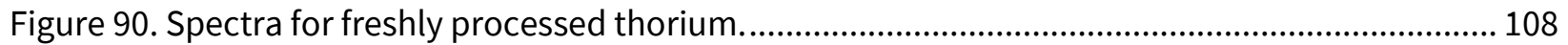

Figure 91. Nuclear transmutation, decay processes, and half-lives of isotopes involved in the thorium fuel cycle.

Figure 92. Analytical plan for (a) isotope dilution where blue represents the tracer and green represents sample isotope ratios. 


\section{Tables}

Table 1. Leading thorium fuel cycles.

Table 2. Basis for the selection of the three leading thorium fuel cycles referencing specific

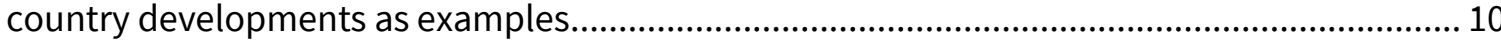

Table 3. Reactor implementation and representative fuel forms for chosen thorium fuel cycles. ........ 19

Table 4. Bounding $\mathrm{UO}_{2}$ PWR fuel compositions used as the baseline against which thorium-

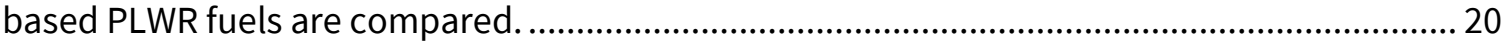

Table 5. Bounding $\mathrm{UO}_{2}-\mathrm{ThO}_{2}$ homogeneous lattice PLWR fresh fuel compositions............................... 22

Table 6. Bounding $\mathrm{UO}_{2}$ heterogeneous lattice PLWR seed fuel compositions. ..................................... 25

Table 7. Bounding $\mathrm{UO}_{2}-\mathrm{ThO}_{2}$ heterogeneous lattice PLWR blanket fuel compositions......................... 26

Table 8. $\mathrm{UO}_{2}-\mathrm{ThO}_{2}$ homogeneous PLWR fuel lattice with ${ }^{233} \mathrm{U}$ makeup................................................... 29

Table 9. $\mathrm{UO}_{2}-\mathrm{ThO}_{2}$ heterogeneous (seed and blanket) PLWR fuel lattice with ${ }^{233} \mathrm{U}$ makeup and

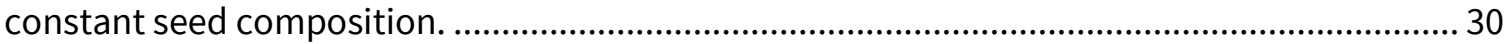

Table 10. $\mathrm{UO}_{2}-\mathrm{ThO}_{2}$ heterogeneous (seed and blanket) PLWR fuel lattice with ${ }^{233} \mathrm{U}$ makeup and

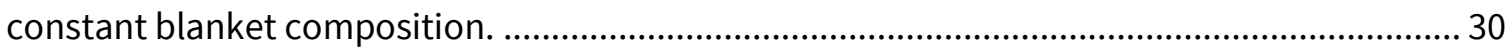

Table 11. $\mathrm{UO}_{2}-\mathrm{ThO}_{2}$ homogeneous PLWR fuel lattice with ${ }^{235} \mathrm{U}$ makeup.............................................. 32

Table 12. $\mathrm{UO}_{2}-\mathrm{ThO}_{2}$ heterogeneous (seed and blanket) PLWR fuel lattice with ${ }^{235} \mathrm{U}$ makeup and

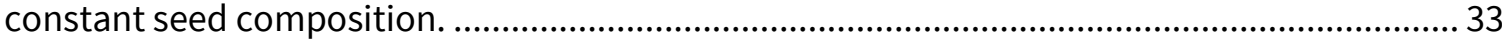

Table 13. $\mathrm{UO}_{2}$ - $\mathrm{ThO}_{2}$ heterogeneous (seed and blanket) PLWR fuel lattice with ${ }^{235} \mathrm{U}$ makeup and

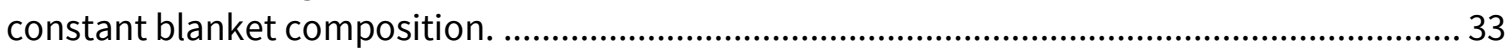

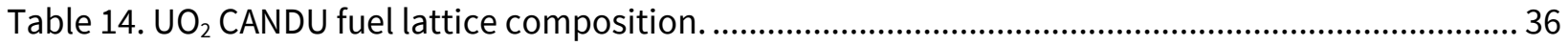

Table 15. Fuel compositions of the AHWR Initial Type I fuel cluster. .................................................... 37

Table 16. Fuel compositions of the AHWR Initial Type II fuel cluster.................................................... 38

Table 17. Fuel compositions of the AHWR Transition Type fuel cluster. ................................................. 38

Table 18. Fuel compositions of the AHWR Equilibrium Type fuel cluster.............................................. 38

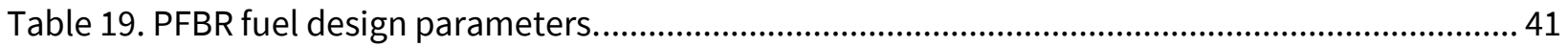

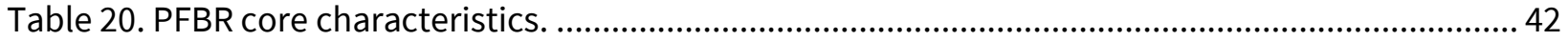

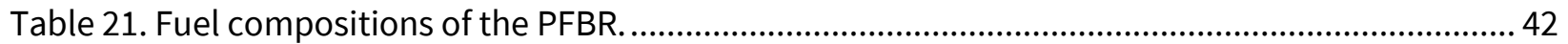

Table 22. Initial fuel compositions of the MSBR fuel salt................................................................... 46

Table 23. Estimated masses of ${ }^{233} \mathrm{U}$ per initial thorium mass in assembly types at discharge................ 48

Table 24. Starting compositions of the four types of AHWR assemblies and a regular $17 \times 17$

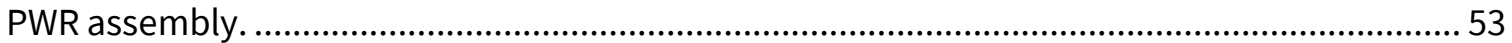

Table 25. Isotopic concentrations (ppm) of the isotopes that could be identified from the simulated 30-day cooling time gamma spectra. ........................................................................ 58

Table 26. Isotopic concentrations (ppm) of the isotopes that could be identified from the

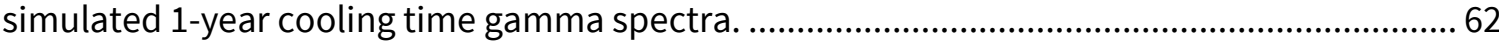

Table 27. Isotopic concentrations ( $\mathrm{ppm}$ ) of the isotopes that could be identified from the simulated 5-year cooling time gamma spectra.

Table 28. Isotopic concentrations (ppm) of the isotopes that could be identified from the simulated 20-year cooling time gamma spectra.

Table 29. Spontaneous fission multiplicities and fission yields of selected thorium, uranium,

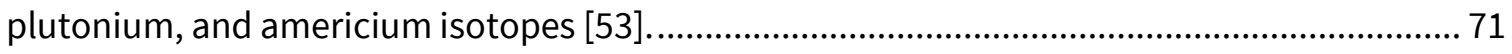

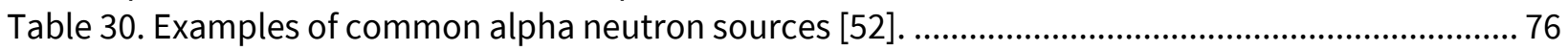


Table 31. Relative comparison of induced fission probability of ${ }^{232}$ Th with respect to ${ }^{233} \mathrm{U},{ }^{235} \mathrm{U}$, and ${ }^{238} \mathrm{U}$ as a function of fast neutron energy (based on evaluated data from ENDF VII [50]).

Table 32. Relative comparison of induced fission probability of ${ }^{233} \mathrm{U}$ and ${ }^{235} \mathrm{U}$ as a function of incident neutron energy (based on evaluated data from ENDF VII [50]).

Table 33. Main characteristics of the plutonium sources used for ENMC and AWCC model validation. 84

Table 34. Simulated singles, doubles, and triples for $\mathrm{PuO}_{2}$ STD 1 (ENMC). 85

Table 35. Simulated singles, doubles, and triples for $\mathrm{PuO}_{2}$ STD 2 (ENMC). 86

Table 36. Calculated singles and doubles for $\mathrm{PuO}_{2}$ STD 1 (ENMC) 87

Table 37. Calculated singles and doubles for $\mathrm{PuO}_{2}$ STD 2 (ENMC) 87

Table 38. Calculated vs. simulated vs. measured count rates for $\mathrm{PuO}_{2}$ STD 1 (ENMC)..........................8 88

Table 39. Calculated vs. simulated vs. measured count rates for $\mathrm{PuO}_{2}$ STD 2 (ENMC)......................... 88

Table 40. ${ }^{252} \mathrm{Cf}$ and AmLi source intensities from the decay-corrected source certificate values........... 88

Table 41. Calculated vs. measured count rates for Cf Source 1 (ENMC)............................................ 89

Table 42. Calculated vs. measured count rates for Cf Source 2 (ENMC).............................................. 89

Table 43. Calculated vs. measured count rates for AmLi Source 1 (ENMC). .......................................... 89

Table 44. Calculated vs. measured count rates for AmLi Source 1 (AWCC) .........................................90

Table 45. Characteristics of the considered thorium sources............................................................... 90

Table 46. Results for passive measurements performed using ENMC ................................................. 91

Table 47. Single count rates for thorium samples interrogated by AmLi Source 1............................. 91

Table 48. Single count rates for thorium samples (AmLi Source 1) ................................................... 92

Table 49. Singles and doubles count rates for a ${ }^{233} \mathrm{U}$-enriched/thorium-bearing sample....................... 93

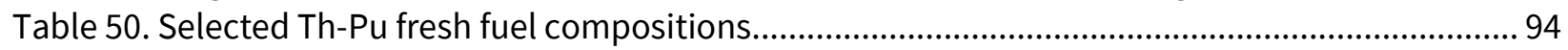

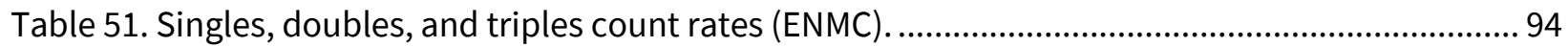

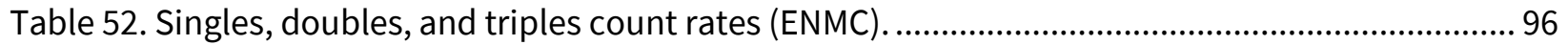

Table 53. Summary of the evaluation of the feasibility of neutron NDA techniques. ........................... 98

Table 54. Unshielded working hours required to accumulate a $5 \mathrm{rem}$ dose $(5 \mathrm{~kg}$ sphere of metal at $50 \mathrm{~cm}$ ) one year after separation [74]. 102 


\section{Acronyms}

\begin{tabular}{|c|c|}
\hline AHWR & advanced heavy water reactor \\
\hline AP & Additional Protocol to Comprehensive Safeguards Agreements \\
\hline AWCC & active well coincidence counter \\
\hline BWR & boiling water reactor \\
\hline $\mathrm{CA}$ & Complementary Access \\
\hline CANDU & CANada Deuterium Uranium \\
\hline CSA & comprehensive safeguards agreement \\
\hline COTS & commercial-off-the-shelf \\
\hline DA & destructive assay \\
\hline D-D & deuterium-deuterium \\
\hline DDSI & differential die-away self-interrogation \\
\hline DIV & design information verification \\
\hline DNN R\&D & Office of Defense Nuclear Nonproliferation Research and Development \\
\hline DOE & US Department of Energy \\
\hline DOE-NE & DOE Office of Nuclear Energy \\
\hline D-T & deuterium-tritium \\
\hline DU & depleted uranium \\
\hline EFPD & effective full-power day \\
\hline ENDF & Evaluated Nuclear Data File \\
\hline ENMC & epithermal neutron multiplicity counter \\
\hline FBR & fast breeder reactor \\
\hline GADRAS & Gamma Detector Response and Analysis Software \\
\hline GPS & global positioning system \\
\hline HEU & high-enriched uranium \\
\hline HPGe & high-purity germanium \\
\hline HWR & heavy water reactor \\
\hline IAEA & International Atomic Energy Agency \\
\hline IF & induced fission \\
\hline INCC & IAEA Neutron Coincidence Counting code \\
\hline ITV & international target value \\
\hline JENDL & Japanese Evaluated Nuclear Data Library \\
\hline LANL & Los Alamos National Laboratory \\
\hline LEU & low-enriched uranium \\
\hline LFTR & liquid fluoride thorium reactor \\
\hline LWR & light water reactor \\
\hline MAVRIC & Monaco with Automated Variance Reduction using Importance Calculations \\
\hline MCIK & Multi Components Inspectors Kit \\
\hline MCNP & Monte Carlo N-Particle Transport \\
\hline MOX & mixed-oxide fuel \\
\hline MSBR & molten salt breeder reactor \\
\hline MSR & molten salt reactor \\
\hline NDA & nondestructive assay \\
\hline NNSA & National Nuclear Security Administration \\
\hline
\end{tabular}




$\begin{array}{ll}\text { NNWS } & \text { non-nuclear-weapon State } \\ \text { NPT } & \text { Treaty on the Nonproliferation of Nuclear Weapons (Nonproliferation Treaty) } \\ \text { NRC } & \text { US Nuclear Regulatory Commission } \\ \text { NWS } & \text { nuclear weapon State } \\ \text { NWAL } & \text { Network of Analytical Laboratories } \\ \text { ORIGEN } & \text { Oak Ridge Isotope Generation and Depletion code } \\ \text { ORNL } & \text { Oak Ridge National Laboratory } \\ \text { PFBR } & \text { prototype fast breeder reactor } \\ \text { PHWR } & \text { pressurized heavy water reactor } \\ \text { PI } & \text { proliferation indicator } \\ \text { PWR } & \text { pressurized light water reactor } \\ \text { Ppm } & \text { parts per million } \\ \text { PWR } & \text { pressurized water reactor } \\ \text { R\&D } & \text { research and development } \\ \text { RIID } & \text { radioisotope identifier } \\ \text { SCALE } & \text { Standardized Computer Analyses for Licensing Evaluation } \\ \text { SF } & \text { spontaneous fission } \\ \text { SNF } & \text { spent nuclear fuel } \\ \text { TCIF } & \text { Time Correlated Induced Fission } \\ \text { Th/U } & \text { thorium/uranium } \\ \text { TMSR } & \text { thorium molten salt reactor } \\ \text { TMSR-LF } & \text { liquid fuel thorium molten salt reactor } \\ \text { TMSR-SF } & \text { solid fuel thorium molten salt reactor } \\ \text { TRITON } & \text { Transport Rigor Implemented with Time-dependent Operation for Neutronic } \\ \text { TRL } & \text { Depletion code } \\ \text { U/Pu } & \text { technology readiness level } \\ \text { VOA } & \text { uranium/plutonium } \\ & \text { voluntary offer agreement } \\ \end{array}$




\section{Acknowledgments}

The authors would like to acknowledge and thank the Safeguards Program of the US Department of Energy National Nuclear Security Administration Office of Defense Nuclear Nonproliferation Research and Development for supporting this 2-year scoping study under project OR18-V-SG Tec Needs Th Fuel Cycles-PD1Lb, "Safeguards Technology Needs Assessment for Leading Thorium Fuel Cycles." The authors would especially like to thank Program Manager Dr. Christopher Ramos for technical guidance and vision, as well as input to this report.

The authors would also like to acknowledge and thank the following extended multilaboratory team for their contributions and support: Dr. Emory Collins and Dr. Jeffrey Powers (Oak Ridge National Laboratory [ORNL]), Dr. Rollin Lakis (Los Alamos National Laboratory) and Cody Lloyd (Y-12 National Security Complex).

Finally, the authors would like to acknowledge and thank Nathan Armistead for graphic design, Laurie Varma for technical editing, and Kathy Jones for report layout (all of ORNL). 


\section{Executive Summary}

This report presents Safeguards Technology for Thorium Fuel Cycles: Research and Development Needs Assessment and Recommendations prepared for the National Nuclear Security Administration (NNSA) Office of Defense Nuclear Nonproliferation Research and Development (DNN R\&D) Safeguards Program by a multilaboratory team from Oak Ridge National Laboratory, Los Alamos National Laboratory, and Y-12 National Security Complex. It documents key findings of a 2-year scoping study on "Safeguards Technology Needs Assessment for Leading Thorium Fuel Cycles" (project OR18-V-SG Tec Needs Th Fuel Cycles-PD1Lb).

\section{Motivation}

Active research into thorium fuels, fuel cycle concepts, and thorium utilization in different reactor types is currently being conducted around the world, including research into the use of thorium in advanced reactor designs. Over six decades, the US Department of Energy (DOE) has spearheaded substantial investment in several advanced reactor concepts including those using thorium. Safeguards technology innovation and research and development (R\&D) are needed to keep pace with emerging thorium nuclear technologies.

Safeguards measures are routinely implemented at existing uranium- and plutonium-based civilian fuel cycle facilities and nuclear reactors to verify nuclear material and facilities remain in peaceful use, as mandated by the Treaty on the Nonproliferation of Nuclear Weapons, commonly referred to as the Nonproliferation Treaty or NPT [1]. Safeguards in-field verification efforts include activities such as review of shipper/receiver records and use of measurement technologies to verify State declarations. The International Atomic Energy Agency (IAEA) Department of Safeguards' strategic objectives include "To deter the proliferation of nuclear weapons, by detecting early the misuse of nuclear material or technology, and by providing credible assurances that States are honoring their safeguards objectives" [2].

To continue to meet the IAEA's international safeguards objectives, the IAEA Department of Safeguards cites the need for "Anticipating and responding to new demands" and "Keeping up with technology and innovating" in their strategic planning documents [2]. Top-priority R\&D needs include "Strengthening instrumentation capabilities for safeguards: Prepare for new types of facilities. Based on the prospects and timing for emerging nuclear facilities, develop and deploy as appropriate: safeguards concepts, tools, techniques, training." Furthermore, on page 27 of the summary recommendations from the 2018 IAEA Symposium on International Safeguards: Building Future Safeguards Capabilities [3], the IAEA cites "Developing verification techniques for the thorium fuel cycle" as being a challenge. A paper on this scoping study [4] was presented at that symposium. In addition, Uribe et al. made a presentation discussing the challenges associated with protactinium-233 $\left({ }^{233} \mathrm{~Pa}\right)$, which is relevant to thorium fuel cycles [5].

Although most of the thorium fuel cycle technologies identified in this study are being developed by NPT-recognized nuclear-weapon States (NWSs) (e.g., China, the United States, UK), active R\&D into thorium fuel cycle technologies is also occurring in non-nuclearweapon States (NNWSs) (e.g., Canada, India). Moreover, these thorium fuel cycles, reactor concepts, and associated nuclear material may 
be exported and operated anywhere in the world. If a nuclear facility is constructed or operated in an NNWS that has a comprehensive safeguards agreement [6] in force with the IAEA, the IAEA would be legally obligated to verify the nuclear material and facility(ies) remain in peaceful use.

Additionally, in the case of NPT NWSs (e.g., the United States), should the civilian facilities or nuclear material be subject to the States' Voluntary Offer Agreement, the IAEA would also have the legal right and obligation to inspect such facilities. Finally, in the case of States that are not party to the NPT (e.g., India), with an item-specific safeguards agreement in place, the IAEA has the legal obligation to verify that nuclear material, facilities, and other items specified under the safeguards agreement remain in peaceful use.

Undertaking technology foresight activities (such as this scoping study) and engaging early with vendors as they design reactor concepts are also consistent with the IAEA and US Government-published policies to promote safeguards by design [7] and could significantly increase the effectiveness of safeguards, as well as reduce future costs to the IAEA, designers, and States.

With active $R \& D$ on thorium fuel cycles occurring throughout the world-from thorium fuels development to full fuel cycle implementation-and policy decisions in some countries geared toward deployment and commercialization of thorium fuel cycles, the goal of this scoping study was to understand the scope, nature, and timescale of R\&D that will be needed to transition or adapt the current safeguards technology toolkit to meet the verification needs of emerging thorium fuel cycles.

\section{Purpose}

The purpose of this report is to provide a technical basis and guide for DNN R\&D to make decisions regarding potential future investments in international nuclear safeguards technology R\&D applied to thorium fuel cycles and associated nuclear technologies (i.e., reactors and supporting fuel cycle facilities). The intention is to help DNN R\&D not only in planning which technology investments should be considered, but also to provide a timeline of when these investments should begin. In an ideal scenario, the timeline required to develop and implement new international nuclear safeguards verification technologies in the field should be less than or equal to the timeline for the anticipated development and deployment of thorium fuel cycles. This R\&D needs assessment reflects the goals of the DNN R\&D Safeguards Program, but also considers the broader goals of the entire DNN R\&D portfolio.

This study has identified leading candidate thorium fuel cycles that are considered the most likely to be fully developed and deployed on an industrial scale in the near term, defined as less than 10 years. This report provides an assessment of the suitability of existing safeguards technologies, which are currently used for uranium- and plutonium-based nuclear fuel cycles, for their application to these leading thorium-based nuclear fuel cycles (i.e., if any of them can be applied directly without further research, need to be modified, or whether new methods or technologies need to be developed). This assessment provides the scientific basis for the recommendations provided regarding strengthening existing instrumentation capabilities or developing new instrumentation that may be needed to fill any potential capability gaps within the international nuclear safeguards community, 
in order to properly verify declarations of any thorium-232 $\left({ }^{232} \mathrm{Th}\right)$ and uranium-233 $\left({ }^{233} \mathrm{U}\right)$ bearing or producing materials (e.g. ${ }^{233} \mathrm{~Pa}$ ) $[6,8]$. This study further highlights how nuclear material typically used in the conventional uranium/plutonium (U/Pu) fuel cycle may also be present in thorium fuel cycles in different chemical or physical forms, which will likely impact the performance of existing safeguards technologies.

\section{Method of Investigation}

The project began with a literature review of the current state of the art of thorium-based nuclear fuel cycle R\&D and, together with the most up-to-date technical State policy decision documents (when available), identified types of leading thorium-based nuclear fuel cycles with their key corporate and/or State proponents. Representative nuclear material inventories and material flows were then identified for each leading thorium-based fuel cycles and simulated using modeling and computational tools within the SCALE (Standardized Computer Analyses for Licensing Evaluation) code package [9, 10]. These activities enabled this assessment to draw analogies between the leading thoriumbased fuel cycles and the most prominent process stages of conventional U/Pu nuclear fuel cycles, where safeguards verification measurements are performed. A theoretical review was then performed of fundamental physical and chemical properties of nuclear material within the leading thorium-based fuel cycles, which was supported by Monte Carlobased neutron and gamma particle transport codes simulating the response of select safeguards detectors in current use by the IAEA, but applied to new scenarios involving the leading thorium fuel cycle-specific nuclear material. Parallel to this theoretical and computational effort, gamma and neutron signatures of various items relevant to and representative of leading thorium-based nuclear fuel cycles were studied experimentally using standard safeguards instrumentation such as the FLIR identiFINDER HM-5 handheld gamma spectrometer [11], high-purity germanium (HPGe) gamma spectrometer, active well coincidence counter (AWCC) and epithermal neutron multiplicity counter (ENMC). Both simulation and experimental results were then qualitatively and quantitatively evaluated for their effectiveness to provide required safeguards verification in a reliable, reproducible, and timely manner. In this regard, methodological and technological gaps were identified, where current safeguards analysis methods and technologies are unable to perform verification measurements, either qualitatively or quantitatively.

\section{Key Findings and Recommendations}

The legal framework of the IAEA is longestablished and does include the right and obligation to implement thorium fuel cycle safeguards. However, historically, much greater attention has been devoted to safeguards concepts, approaches, and measures (i.e., technologies and activities to enable the IAEA to meet technical verification objectives) for uranium- and plutonium-based fuel cycles than for thorium-based fuel cycles. Therefore, emerging technologies specific to thorium fuel cycles will likely require modified safeguards approaches to meet new and unique technical objectives, or to meet the same technical objectives in new ways.

Additionally, the IAEA may have to adjust some of its policies and practices to verify all nuclear material in thorium-based fuel cycles remain in peaceful use, maintaining effective and efficient safeguards implementation. For example, ${ }^{233} \mathrm{~Pa}$, which is neither fissile nor fertile, is currently not defined as a nuclear material under IAEA safeguards. However, it is a direct precursor to ${ }^{233} \mathrm{U}$, which is a nuclear 
material, and some thorium-based fuel cycle designs include separation of ${ }^{233} \mathrm{~Pa}$ from the process streams to decay into ${ }^{233} \mathrm{U}$. The IAEA may need to know quantities of separated ${ }^{233} \mathrm{~Pa}$ to effectively verify quantities of ${ }^{233} \mathrm{U}$. Three leading thorium fuel cycles were identified due to their highest likelihood of deployment and greatest level of international interest, which are described in Section 1. These three leading thorium fuel cycles represent different evolutions of thorium implementation in four different reactor types, where the multistage fuel cycle has two reactor types. Section 2 provides details regarding the fuel cycle and reactor simulations undertaken in this study. Technology R\&D needs are discussed in detail in Section 3. Section 4 summarizes safeguards policy areas for consideration. Section 5 summarizes the technology $R \& D$ needs detailed in Section 3. The three leading fuel cycles considered in this study are:

1) Thorium utilization in a multistage fuel cycle with continuous recycle of ${ }^{233} \mathrm{U}$, such as that implemented in heavy water reactors (e.g., CANDU-type [CANada Deuterium Uranium]), and fast reactors in the Indian nuclear energy development program.

2) Thorium utilization in a once-through fuel cycle, or fuel cycle with continuous recycle, in a pressurized water reactor (PWR), such as the thorium-plutonium mixed-oxide fuel bundles for use in PWRs developed by Thor Energy in Norway.

3) Thorium utilization in a fuel cycle with continuous recycle of ${ }^{233} \mathrm{U}$ in a molten salt reactor (MSR), such as the liquid fuels in the Chinese Thorium Molten Salt Reactor program, the US-based Flibe Energy Inc.'s Liquid Fueled Thorium Reactor, and the U.S.-Indonesia ThorCon MSR concept.

\section{Why are thorium fuel cycles unique?}

Thorium fuel cycles have some pertinent and distinct characteristics that make them unique relative to other fuel cycles:

- ${ }^{232} \mathrm{Th}$ is a fertile isotope, leading to the production of ${ }^{233} \mathrm{U}$ from neutron capture reactions during the irradiation of ${ }^{232} \mathrm{Th}$ in a nuclear reactor. Therefore, a "fissile driver" is first required for a nuclear reactor to start operating on a thorium fuel cycle. This means that fissile ${ }^{233} \mathrm{U},{ }^{235} \mathrm{U}$, or plutonium-239 or plutonium-241 $\left({ }^{239,241} \mathrm{Pu}\right)$ must always be present within fresh nuclear fuel, such as a fresh fuel assembly or unirradiated salt fuel.

- ${ }^{233} \mathrm{U}$ production from the reactor irradiation of ${ }^{232} \mathrm{Th}$ in thorium fuel cycles is analogous to the reactor production of ${ }^{239} \mathrm{Pu}$ from ${ }^{238} \mathrm{U}$ in conventional fuel cycles.

- From a safeguards perspective, in many (but not all) aspects, ${ }^{232} \mathrm{Th}$ is analogous to ${ }^{238} \mathrm{U}$, while ${ }^{233} \mathrm{U}$ is analogous to ${ }^{239} \mathrm{Pu}$. There is no straightforward analog of ${ }^{235} \mathrm{U}$. On one hand, ${ }^{233} \mathrm{U}$ among ${ }^{232} \mathrm{Th}$ in fresh thorium fuel may be analogous to ${ }^{235} \mathrm{U}$ among ${ }^{238} \mathrm{U}$ in fresh uranium fuel. However, we have found that it might be difficult to distinguish between ${ }^{235} \mathrm{U}$ and ${ }^{233} \mathrm{U}$ under many distinct measurement conditions due to their similar neutron-induced fission cross-sections across the entire energy range.

- ${ }^{233} \mathrm{U}$ has a smaller critical mass than ${ }^{235} \mathrm{U}$, and its IAEA recognized significant quantity is $8 \mathrm{~kg}$, which is identical to that of plutonium ( $8 \mathrm{~kg}$ for Pu containing less than $80 \%{ }^{238} \mathrm{Pu}$ ). The significant quantity for thorium is 20 metric tons, which is identical to that for depleted uranium with an enrichment of $0.5 \%$ or lower [8]. 
- Existing reactor design concepts produce an almost-pure stream of ${ }^{233} \mathrm{U}$ (i.e., a separated, unirradiated stream of ${ }^{233} \mathrm{U}$ of such quality that it is considered direct-use material), either within the reactor facility or in a separate reprocessing facility.

- $\quad{ }^{233} \mathrm{U}$ recovered from spent nuclear fuel has often been generally considered "selfprotecting" due to the presence of ${ }^{232} \mathrm{U}$ and its daughter radionuclide thallium-208 $\left.{ }^{208} \mathrm{Tl}\right)$, which is a strong gamma emitter that could result in significant health risks to a potential human diverter. However, the concept of self-protection is not relevant to international safeguards. Furthermore, such an approach is questionable, even for domestic safeguards when considering a truly determined rogue actor. Moreover, within certain thorium fuel cycles, a pure stream of ${ }^{233} \mathrm{U}$ can be produced, which reduces the quantity of ${ }^{232} \mathrm{U}$ present and thus the radiation-associated health hazard.

- Contamination of ${ }^{233} \mathrm{U}$ by ${ }^{232} \mathrm{U}$ may have significant impact on the ability of human inspectors to perform safeguards verification measurements and may require remote inspections with robotic inspection units. Hence, certain techniques, allowed by the fundamental physics principles, may be affected by engineering measures designed for radiation protection of the operators and their workforce.

- The reactor physics of ${ }^{\text {nat } T h / 233} \mathrm{U}$ means that achieving a breeding ratio greater than one is possible in both thermal and fast reactors utilizing thorium, as opposed to only fast reactors that operate in a U/Pu fuel cycle. In other words, there is potential for certain reactor types to produce more ${ }^{233} \mathrm{U}$ than is needed for their sustained operations.
- Thorium is more abundant than uranium but is less likely to be concentrated by mineralization. Natural thorium consists of ${ }^{232} \mathrm{Th}$ with small amounts of ${ }^{230} \mathrm{Th}$. Usually, the ${ }^{230} \mathrm{Th}$ is present in trace amounts (typically $\sim 30 \mathrm{ppb}$ ) due to the decay of ${ }^{238} \mathrm{U}$ and, subsequently, ${ }^{234} \mathrm{U}$.

Within this scoping study, we have found that:

- The thorium fuel cycle is significantly more diverse with regard to the nuclear material types included in existing designs and design concepts, including multiple fertile and fissile isotopes, and with regard to the physical and chemical composition of these materials, than in conventional U/Pu fuel cycles. Thus, the technical requirements for verification measurements, and the measured nuclear material signatures, are anticipated to be equally more diverse.

- Nondestructive assay (NDA), qualitative and quantitative, of both ${ }^{232} \mathrm{Th}$ and ${ }^{233} \mathrm{U}$ is generally possible and straightforward for some applications, but is technically challenging under a significant number of relevant scenarios, and is not always practically feasible with current state-ofthe-art technologies used in international safeguards as they are applied today.

- Nuclear material in several thorium-based reactor design concepts would be highly heterogeneous and consist of multiple fissile and/or fertile isotopes. Identifying and quantifying individual isotopes within these materials poses a significant challenge. Recommendations provided within this report aim to address these shortcomings.

- One of the greatest general NDA challenges with respect to ${ }^{232} \mathrm{Th}$ is quantitative assay of its total mass. 
- One of the greatest general NDA challenges with respect to ${ }^{233} \mathrm{U}$ is associated with its prompt identification, particularly if significantly shielded.

- In principle, destructive assay (DA) appears to be straightforwardly extendable to quantifying relative isotopic fractions of ${ }^{232} \mathrm{Th}$ and ${ }^{233} \mathrm{U}$. However, in scenarios where conventional U/Pu fuel cycle isotopes may also be present (e.g., swiping fresh Th/U/Pu fuel rods), DA may require additional preparation steps due to the potential need to process samples with two different tracers, thus adding to the complexity of the conduct of operations and associated laboratory infrastructure. Two different tracers may be needed when the isotope of interest (i.e., analyte) for thorium fuel cycles is an isotope that is typically used as a tracer for U/Pu fuel cycles (e.g., $\left.{ }^{233} \mathrm{U}\right)$.

- The current Complementary Access (CA) Toolkit used by the IAEA utilizes FLIR's identiFINDER HM-5 detector [11] as its primary, and at times sole, radiation detection instrument. If used under standard conduct of operations, this detector is not capable of discriminating ${ }^{232} \mathrm{Th}$ from ${ }^{232} \mathrm{U}$, nor identifying shielded ${ }^{233} \mathrm{U}$. This is in part due to the lower resolution offered by the sodium iodide detector versus other gamma detector types, as well as the gamma peak analysis software installed by FLIR to evaluate gamma spectra and thus identify individual isotopes of interest. (The software is not currently tailored to thorium fuel cycle items and the measurement scenarios/challenges discussed.) To enable these capabilities, the CA Toolkit may be required to adopt portable/handheld highresolution HPGe detectors, the HM-5 may require modifications to its design and/or capabilities, and/or IAEA safeguards inspectors may need to consider modifying their operations.

Given the findings of this study, we propose to start addressing the gap in technological capabilities of current safeguards methods and instrumentation, preferably (but not necessarily) concurrently, on three different levels:

1) Verify feasibility of basic methods and generic technologies

(to establish a complete set of tools that can be deployed to address all principle thorium fuel cycle safeguards needs)

- Self-interrogation capability of ${ }^{233} \mathrm{U}$ in certain common chemical forms

- Active interrogation of ${ }^{232} \mathrm{Th}$ with a neutron generator or high-energy $(a, n)$ radioisotopic neutron source

- Neutron signature-based discrimination of ${ }^{233} \mathrm{U}$ and ${ }^{235} \mathrm{U}$

- Active interrogation with progressively modulated neutron spectrum to interrogate different energy regions of the neutron-induced fission crosssection

2) Quantify sensitivity limits (to provide the foundation for realistic expectations with respect to the implementation of safeguards techniques in a real environment)

- DA of environmental samples with traces of all major uranium isotopes $\left({ }^{232-238} \mathrm{U}\right)$

- NDA (both gamma and neutron) of pure as well as composite items and in the presence of accidental or deliberate interferences

3) Technological upgrade and/or modification

(to prepare individual instruments and methods for actual deployment) 
- Automated identification and age dating of ${ }^{233} \mathrm{U}$ with gamma methods

- Neutron detector resiliency in highgamma background environment (due to the presence of ${ }^{232} \mathrm{U}$ )

- Active interrogation-based systems using neutron generators, high-energy isotopic sources, or dual-energy interrogation

The list of proposed areas for potential DNN $R \& D$ future research efforts should not be considered exclusive, but rather as a starting point of activities where results may prescribe or inspire direction and scope of further investment and R\&D.

While the authors of this study are convinced that enough subject matter expertise and technological infrastructure exists across the DOE NNSA complex, it is recognized that one primary challenge that needs to be addressed is the lack of relevant nuclear material standards for detection system and method development and calibration. Any systematic progress, especially in terms of experimental verification of proposed or developed safeguards methods (i.e., techniques and equipment) will require a sufficiently diverse, representative, and well-characterized set of relevant thorium fuel cycle nuclear material standards. These materials will need to be sustained in some shape or form for a period of multiple years while developmental work is completed. We recommend that relevant nuclear materials and standards be leveraged from the forensics community [12] and the prior DNN R\&D ${ }^{233} \mathrm{U}$ Preservation project [13] to begin building experimental capability.

A full explanation of these recommendations is provided in Section 5 of this report.

The infographic below summarizes this scoping study on "Safeguards Technology for Thorium Fuel Cycles: Research and Development Needs and Recommendations. 


\section{THE THREE LEADING CANDIDATE THORIUM FUEL CYCLES}

These thorium fuel cycles define the likely scenarios for safeguards verification activities.

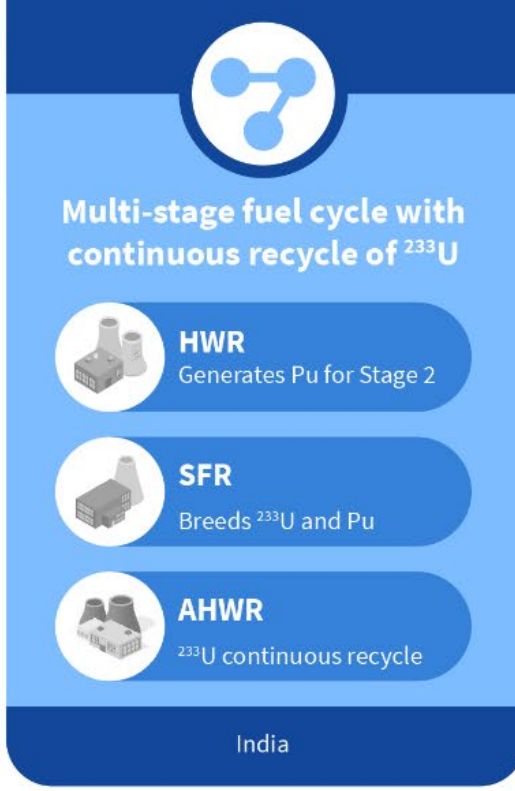

\section{A Safeguards challenges}

Thorium fuel cycles are unique and different from conventional U/Pu fuel cycles. Key differences include (1) greater diversity of nuclear material types and forms; (2) complex isotopic mixtures and interferences; (3) higher fissile enrichment for ${ }^{232}$ Th fuels in thermal spectrum reactors; (4) thorium thermal breeders; and (5) ${ }^{233}$ Pa production of ${ }^{233} \mathrm{U}$, whereby a stream of separated, unirradiated ${ }^{233} \mathrm{U}$ may be produced in some fuel cycles.

Additionally, safeguards verification technologies are tailored for U/Pu fuel cycles. Therefore, isotope identification and quantification challenges will arise from the deployment of Th fuel cycles. Measurement challenges specific to nondestructive assay (NDA) include quantitative NDA of ${ }^{232}$ Th total mass, quantitative NDA of ${ }^{233} \mathrm{U}$ total mass in the presence of

${ }^{232} \mathrm{U}$ and ${ }^{235} \mathrm{U}$, and prompt identification of ${ }^{233} \mathrm{U}$ using NDA (particularly if shielded).
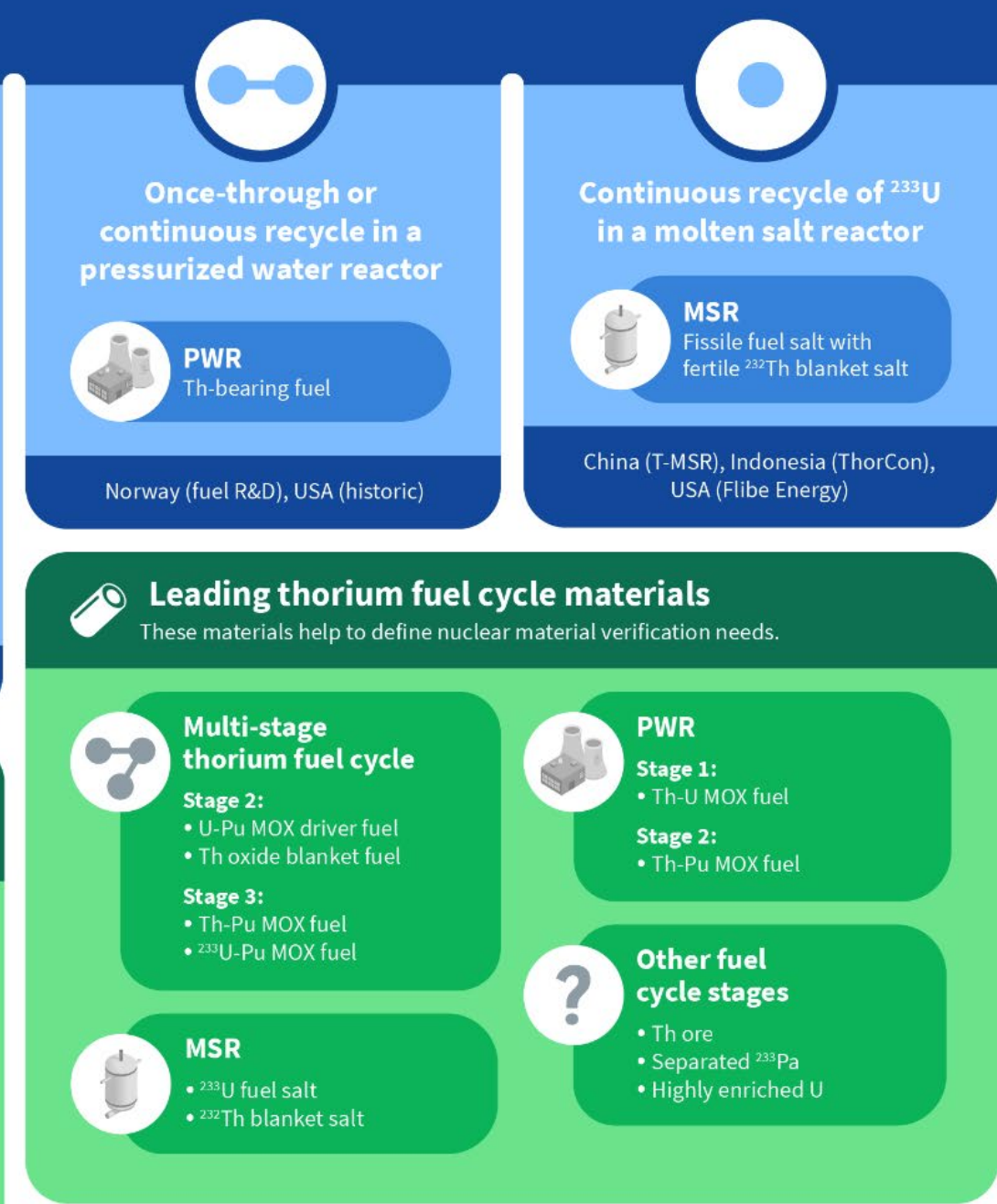

\section{Key findings}

2 ${ }^{232} \mathrm{Th}$ and ${ }^{233} \mathrm{U}$ usually only occur with other nuclear materials.

? From a detection standpoint, ${ }^{232} \mathrm{Th}$ is similar to ${ }^{238} \mathrm{U}$, and ${ }^{233} \mathrm{U}$ is similar to ${ }^{239} \mathrm{Pu}$.

? ${ }^{233} \mathrm{U}$ is difficult to distinguish from ${ }^{235} \mathrm{U}$ using neutron interrogation and detection.

$?$ Due to its high $\alpha$-decay rate, ${ }^{233} \mathrm{U}$ has the potential for neutron self-interrogation using $(\alpha, n)$ neutrons as the driver.

Q ${ }^{23} \mathrm{U}$ has a complex gamma-ray spectrum, low gamma intensities, and high associated background signature from ${ }^{23} 2 \mathrm{U}$ daughter radionuclides.

The HM-5 detector is not capable of discriminating ${ }^{232}$ Th from ${ }^{232} \mathrm{U}$, nor identifying shielded ${ }^{233} \mathrm{U}$.

AHWR = advanced heavy water reactor $\mid$ HWR $=$ heavy water reactor

$\mathbf{M S R}=$ molten salt reactor $\mid \mathbf{P W R}=$ pressurized water reactor $\mid \mathbf{S F R}=$ sodium fast reactor 
This page intentionally left blank. 


\section{Introduction}

With ever-increasing demands placed on available energy resources by a growing human population, and the prospect of accelerating impacts due to restrictions on increased usage of fossil fuels, many nations are pursuing investment into reliable and clean energy-generating technologies including nuclear power. As a result, various advanced nuclear fuel cycles and reactor technologies are also being developed, including thorium fuel cycles.

Many existing or aspiring nuclear nations continue to invest in the development of conventional uranium- or plutonium-based nuclear fuel cycle technologies, while others have chosen to pursue thorium-based nuclear technologies for varying reasons [14]. The primary potential advantages of using thorium include resource utilization, economics, fuel performance characteristics, waste management, and safety. For example, thorium is on average three times more abundant in nature than uranium. However, thorium is less likely to be concentrated by mineralization. Nevertheless, thorium is attractive to pursue as a fuel cycle option for countries with large thorium reserves and limited access to natural uranium deposits or enriched uranium markets [15]. Ade et al. [16] point out that the use of thorium fuels complements uranium and helps ensure the long-term sustainable use of nuclear power. Ultimately, the use of thorium fuel, in addition to uranium fuel, will increase the strategic reserve of nuclear fuel.

This pursuit of thorium-based energy matters from a safeguards and proliferation detection perspective because thorium is a nuclear material, and uranium-233 $\left.{ }^{233} \mathrm{U}\right)$ is also a nuclear material produced during the reactor irradiation of thorium-232 (232Th).
Furthermore, thorium fuel cycles are complex and different from conventional uranium- and plutonium-based fuel cycles. These differences include the nuclear material produced (i.e., ${ }^{233} \mathrm{U}$ ), together with its level of purity; diverse fuel cycle technologies and associated reactor technologies (e.g., advanced reactors and, therefore, diverse fuel forms); and the need for a fissile driver for fertile fuel, leading to the need for supplemental ${ }^{235} \mathrm{U}$ and plutonium-239 $\left({ }^{239} \mathrm{Pu}\right)$ production or acquisition to support production of ${ }^{233} \mathrm{U}$.

Like conventional U/Pu fuel cycles, thorium fuel cycles lead to production and utilization of large quantities of nuclear material with potential use for military purposes. Therefore, in accordance with the spirit and legal framework of the Treaty on the Nonproliferation of Nuclear Weapons (commonly referred to as the Nonproliferation Treaty, or NPT), and the subsequently developed safeguards regime, nuclear material used in thorium-based fuel cycles is required to be under safeguards in NPTdefined non-nuclear weapons States (NNWSs). While this expectation is not new, research and development (R\&D) needs arise because safeguards technologies currently deployed for verification of nuclear material are optimized for conventional uranium- and plutonium-based fuel cycles and the associated isotopes in fuel cycle materials. As such, these technologies typically utilize specific neutron and gamma radiation signatures-as well as non-nuclear signatures such as mass-of isotopes of interest (e.g., ${ }^{235} \mathrm{U}$, ${ }^{239} \mathrm{Pu},{ }^{240} \mathrm{Pu}$,), which when analyzed together can provide verification of the specific isotopic composition of fuel cycle material, as well as the quantity and form of nuclear material present. Distinguishing isotopes (e.g., ${ }^{232} \mathrm{Th}$, ${ }^{233} \mathrm{U},{ }^{232} \mathrm{U}$ ) that are very dilute in 
uranium/plutonium (U/Pu) fuels, are orders of magnitude more concentrated in thorium fuels. These isotopes have fundamentally different radiation signatures, and it is neither obvious nor guaranteed that instrumentation used for safeguards of conventional nuclear fuel cycles backed by decades of development will perform with the same efficiency and effectiveness for safeguards of thorium nuclear fuel cycles.

\section{Thorium Fuel Cycle Technology}

It is worth noting that there is no such thing as a single thorium fuel cycle because many thorium fuel cycle options exist. Thorium fuel cycles differ from conventional uranium and plutonium fuel cycles in the nuclear materials present, fuel forms, and processes. Thorium fuels can be utilized in a variety of nuclear reactor types, either in existing commercial reactors (e.g., light water reactors [LWRs]) or in advanced reactors (e.g., molten salt reactors [MSRs], fast reactors). The reactor physics of Th- ${ }^{233} \mathrm{U}$ means that breeding is possible in thermal reactors. The $\mathrm{Th}^{233} \mathrm{U}$ fuel cycle requires separation and recycle of fissile material to give maximum resource utilization and thus maximum benefit, similar to the U/Pu fuel cycle. However, thorium reprocessing is less mature than current reprocessing and has not been demonstrated on a commercial scale. In addition, the presence of ${ }^{232} \mathrm{U}$ makes fuel refabrication more difficult.

Thorium fuel cycles have several potential (claimed) benefits relative to U/Pu fuel cycles [17]:

- Thorium is an alternative strategic energy resource to uranium and thus provides a diversification option for nuclear fuel supply [18].

- Thorium fuel cycles have thermal breeding capability.
- Spent fuel from thorium fuel cycles has lower radiotoxicity over time.

- Thorium fuel has potentially improved proliferation resistance due to the presence of ${ }^{232} \mathrm{U}$. However, self-protection should not be considered under international safeguards, and ${ }^{232} \mathrm{U}$ is also present at varying levels depending on reactor design and operating parameters and reprocessing scheme.

- Due to its higher thermal conductivity, during normal operations, $\mathrm{ThO}_{2}$-based fuel will operate with lower fuel temperatures and release less fission gas than $\mathrm{UO}_{2}$ fuel at corresponding powers and burnups. This may allow for reduced fuel-cladding interactions and higher burnup use [19].

- Mixed $\mathrm{ThO}_{2}-\mathrm{UO}_{2}$ is more resistant to longterm corrosion in air or oxygenated water than $\mathrm{UO}_{2}$ alone. Thus, $\mathrm{ThO}_{2}-\mathrm{UO} 2$ is a superior waste form if the spent fuel is slated for direct disposal rather than reprocessing [19].

Some of these potential benefits are subject to various stakeholders' interpretation and prioritization. Some do not consider variations in different thorium fuel cycles. While the purpose of this study is not to review individual benefits claimed, we feel compelled to comment on perceived proliferation resistance. It has been long debated whether spent fuel of conventional U/Pu fuel cycles is self-protecting against misuse by a determined bad actor. Similar discussion also applies to spent fuel from thorium fuel cycles. Perhaps more importantly, certain thorium fuel cycles allow for production of ${ }^{23} \mathrm{U}$ essentially free of ${ }^{232} \mathrm{U}$ (such as the fast breeder reactors and MSRs discussed in Sections 2.3.3 and 2.3.4, respectively), in which case the self-protecting argument cannot be made. 


\section{Mission Relevance:}

\section{Proliferation Concerns and}

\section{Challenges}

Despite their attractiveness from a commercial and energy security standpoint, thorium fuel cycles present new and unique safeguards and nonproliferation concerns and challenges. These arise primarily from, but are not limited to, separated ${ }^{233} \mathrm{U}$, which is a direct-use fissile material, as well as the fact that thorium fuel cycle technology may be exported. Potential proliferation detection risks are driven by the fact that currently all nuclear safeguards and security systems are optimized for the detection of other isotopes of uranium and plutonium, which aspiring proliferators could potentially exploit. Additionally, fundamentally different technological process modalities (e.g., MSRs) result in vastly different facility footprints, which may yield significant implications both for safeguards implementation and remote detection.

From a legal perspective, separated, fissile ${ }^{233} \mathrm{U}$ is recognized by the International Atomic Energy Agency (IAEA) as a direct-use nuclear material and is required to be under safeguards. A significant quantity of ${ }^{233} \mathrm{U}$ is the same as a significant quantity of $\mathrm{Pu}(8 \mathrm{~kg})$. This is smaller than a significant quantity of highenriched uranium (HEU), or $>20 \%$ of ${ }^{235} \mathrm{U}$, which is $25 \mathrm{~kg}$ [3]. Thorium is also required to be under safeguards. Natural thorium (mostly ${ }^{232} \mathrm{Th}$ ) is recognized by the IAEA as indirect-use nuclear material, with a significant quantity of 20 metric tons [8]. From a safeguards perspective, the IAEA assumes natural thorium to be only ${ }^{232} \mathrm{Th}$; however, as previously described, trace levels of ${ }^{230} \mathrm{Th}$ are usually present.

In addition to challenges arising from the two isotopes that are unique to thorium fuel cycles, relatively large quantities of traditional nuclear material may also be used (e.g., low-enriched uranium [LEU], HEU, Pu) within these fuel cycles, but in materials and processes that the traditional safeguards community is less familiar with, which may pose additional safeguards challenges.

For the reasons outlined, investment in proliferation detection R\&D and innovation in safeguards instrumentation is required to address challenges associated with new and advanced fuel cycle technologies being developed around the world, especially with respect to thorium fuel cycles, one of the most prominent new technologies. Considering the timeline of development of proposed thorium fuel cycles, together with timelines typical for development and implementation of novel safeguards technologies, now is the time to initiate safeguards technology and proliferation detection assessments at the design phase.

Significant investment into $R \& D$ of various types of thorium fuel cycles has been under way for some time, including, but not limited to, MSRs and thorium fuel development. Active programs in thorium fuel development and thorium fuel cycle and reactor deployment are already well established around the world, particularly in Canada, China, India, Norway, and the United States. Test reactors and the associated fuel cycle infrastructure already exist and will likely be ready for industrial scale deployment in the next 10-15 years. With such global growth in thorium utilization, the potential for growth of commercial thoriumbased nuclear technologies seems ever more likely, thus increasing the probability of thorium fuel cycle deployment in additional nation states, even if a State does not currently participate in its own thorium fuel cycle R\&D activities. Without technically adequate safeguards protocols and verification measures (e.g., appropriate safeguards technology) in place, any future potential clandestine misuse of these fuel cycles could 
go undetected, eventually compromising the deterrent value of the safeguards regime as a whole. The development of safeguards approaches and, specifically, safeguards technology for thorium fuel cycles is therefore a matter of ever-increasing urgency, as the timeline for technology development from initial conception through research, development, testing, demonstration, and deployment is significantly long. Yet, the focus of the international safeguards community nearly exclusively remains on safeguarding conventional ${ }^{235} \mathrm{U}$ - and ${ }^{239} \mathrm{Pu}$-based fuel cycles while the safeguards technology challenges facing thorium fuel cycles to date remain largely uninvestigated and, to a great degree, also underappreciated.

\section{Project Scope}

As described, several thorium fuel cycle variants are being actively pursued by the world nuclear energy community. These variants have short-, medium-, and long-term deployment pathways in a variety of reactor types, which in turn give rise to a variety of fuel designs, fuel cycle facilities, and nuclear material processing requirements. These emerging fuel cycles will impact the technical implementation of safeguards and already are raising questions about the applicability of current verification technologies. To help bridge the anticipated safeguards technology gap, this report introduces the safeguards technology needs for leading thorium fuel cycles. It also presents the science and technology needed to transition, where possible, the current safeguards technology toolkit from a focus on ${ }^{235} \mathrm{U}$ and ${ }^{239} \mathrm{Pu}$ detection to detection of ${ }^{233} \mathrm{U}$ and other signatures relevant to meet the verification needs of thorium fuel cycles. Assessment of current safeguards technologies includes understanding whether safeguards technology, as with U/Pu fuel cycles, can be applied "out of the box," whether current safeguards technology needs to be modified, and whether new safeguards technology needs to be developed.

Together with the body of knowledge compiled in the References and Bibliography sections of this report, results of this study can be used to guide and establish priorities for future directed $R \& D$ that is needed to bring the technology readiness levels (TRLs) of safeguards detection methods and instrumentation in line with the higher TRLs of the leading thorium fuel cycles. Therefore, ultimately, this report can serve as a roadmap to ensure that the technical capability of safeguards technology and level of awareness is in step with thorium fuel cycle deployment timescales.

The major research questions explicitly or implicitly answered within this report are listed on the following page. A cross-reference is provided to the sections where those questions are answered. These research questions provide the basis of the assessment of safeguards technology R\&D needs for thorium fuel cycles and, therefore, establish potential solutions or recommendations to help prepare for the verification challenges associated with these nuclear fuel cycles. 
- What are the anticipated timelines for the commercial implementation of thorium fuel cycles? (Section 1)

- What are the leading thorium fuel cycles? Which thorium fuel cycles are the most promising and relevant? (Section 1 )

- What are the material forms and compositions that will need to be safeguarded throughout these fuel cycles? (Section 1)

- What are the key features (e.g., material throughput, radiation signatures) of those fuel cycles relevant to safeguards and, therefore, necessary to provide input to safeguards technology requirements? (Sections 1 and 2)

- Where is fissile material located within the most promising thorium fuel cycles, and how does this mass transform and flow? (Sections 1 and 2)

- What fuel cycle items will need to be measured for safeguards purposes? (Section 1)

- What is the dose to instrumentation in the proximity of a given fuel cycle facility or item subject to assay? (Section 2)

- What radiation signatures are associated with thorium fuel cycles and available for measurement? (Sections 2 and 3 )

- What measurements will need to be performed for safeguarding thorium fuel cycles? (Section 3)

- How well known are these signatures? What are the potential interferences, if any? (Section 3)

- Does detection technology exist to measure these signatures, or does a new technology need to be developed? (Sections 3 and 5)

- Does sufficiently accurate nuclear data exist? (Sections 3 and 5)

- What are the anticipated variations in safeguards implementation for thorium fuel cycles compared with conventional uranium and plutonium fuel cycles? (Section 4)

\section{Audience}

The primary audience for the Assessment of Safeguards Technology R\&D Needs for Thorium Fuel Cycles is the US Department of Energy (DOE) National Nuclear Security Administration (NNSA) Office of Defense Nuclear Nonproliferation Research and Development (DNN R\&D) and, in particular, its Safeguards Program to help set priorities for future investments in safeguards technology and proliferation detection tool development for thorium fuel cycles.

Other key stakeholders for the results of this research include members of broader nuclear safeguards and nonproliferation communities such as organizations and agencies tasked with nuclear safeguards implementation on both the national and international levels (e.g., the IAEA, European Atomic Energy Community, DOE NNSA, US Nuclear Regulatory Commission) as well as commercial entities and trade organizations with an interest in the development and subsequent deployment of any thorium fuel cycle. Moreover, technical results may become an integral part of future safeguards by design assessments thus benefitting, among others, US reactor vendors marketing their technologies to the international community.

\section{Key Terminology}

Throughout this report, two key termsthorium fuel cycle and thorium safeguards technology-are frequently used and are defined here for clarity.

Thorium fuel cycle refers to an entire nuclear fuel cycle in which at least one of its stages utilizes or depends on the use of natural thorium or ${ }^{233} \mathrm{U}$ created by thorium irradiation. There is no single thorium fuel cycle based on one thorium fuel form or reactor type (e.g., MSRs). Instead, there are multiple 
thorium fuel cycles utilizing thorium and ${ }^{233} \mathrm{U}$ in many different physical and chemical forms. Note that certain stages of thorium fuel cycles may not involve thorium or ${ }^{233} \mathrm{U}$ at all (e.g., the production of fissile driver fuel in a multistage fuel cycle with continuous recycle of ${ }^{233} \mathrm{U}$ ).

Thorium safeguards technology, in a general sense, refers to the measurement instrumentation and technological measures used for nuclear material accountancy. This includes the entire end-to-end technology package itself together with radiation detectors or measurement instrumentation (e.g., mass spectrometers), data acquisition instrumentation, data processing and storage, and data analysis and interpretation software. Thorium safeguards technology is any physical or computational verification technology or method applied to the assay or assessment of nuclear material for safeguards purposes-by its physical or chemical form, or by the nature of the process in which it is used-unique to one or more thorium fuel cycles. Containment and surveillance technologies are also considered to be a necessary part of thorium safeguards technology; however, they are not studied here. 


\section{Leading Thorium Fuel Cycles}

To formulate the scientific basis for building new instrumentation to fill any potential detection capability gaps for thorium fuel cycles, the nuclear material types and forms, and their fundamental signatures (including radiation), must first be understood. Understanding the origin and behavior of the fundamental signatures depends on an in-depth understanding of the relevant reactor and fuel cycle technology as well as nuclear material processing capabilities. Therefore, this work began by engaging fuel cycle, reactor, fuel design, and processing experts to define several proposed thorium fuel cycle options and identify the leading candidate thorium fuel cycles with the highest likelihood of commercial implementation. This down-selection was performed to understand the associated nuclear material types and forms and to establish the radiation signatures.

Safeguards technology development for leading thorium fuel cycles requires a technical understanding of the fuel cycles to which safeguards measurements will be applied. For example, safeguards technology requirements are dictated by the chosen thorium fuel cycle and the stages of that fuel cycle, including the isotopic composition and physical/chemical form of the nuclear material item being measured, as well as any specific nuclear material processing capabilities for which the surrounding signatures and environment need to be considered when scoping the safeguards technology requirements to perform a given measurement. Therefore, prior to performing a sciencebased needs assessment of safeguards technology capabilities for application to thorium fuel cycles, it was critical to understand the details of the relevant thorium fuel cycles being developed around the world. Hence, the objective of this initial scoping, described in Section 1, was to determine the leading thorium fuel cycles. Furthermore, it was essential to include a diverse range of thorium fuel cycles in this evaluation. Although the thorium MSR design is sometimes perceived as synonymous with thorium fuel cycles in the context of nonproliferation and international nuclear safeguards, there are other thorium fuel cycle options, including the use of thorium fuels in LWRs, heavy water reactors (e.g., pressurized heavy water reactors [PHWRs]), and more advanced designs, with the MSR being just one example.

This section details the findings of the literature review on active R\&D related to thorium fuel cycles and describes the method by which three leading thorium fuel cycles were selected for further evaluation. The three leading fuel cycles, which provide input to the simulation models explained in Section 2, are also described. The material compositions are defined to provide the basis for evaluating the feasibility of providing safeguards-relevant assays using instruments and methods developed for conventional U/Pu fuel cycles. This evaluation is described in Section 3 and was performed using fundamental physics principles, high-fidelity simulations, and experimental confirmation when possible.

\subsection{Selection Method}

For this work, we selected the most promising thorium fuel cycle options warranting further study, herein referred to as the leading thorium fuel cycles, from those being pursued internationally today. Detailed safeguards analyses and technology evaluations were completed on these leading fuel cycles, as described in Sections 3 and 4. The research and evaluation metrics were based on a stateof-the-art DOE Office of Nuclear Energy (DOE-NE) evaluation of all potential fuel cycles including 
thorium [20]. That study provides the foundation for this research and was combined with a literature review of the international state of play. This step ensured that this project and subsequent findings are relevant to the current international direction of thorium fuel cycles and that the range of options being evaluated is not constrained to narrow safeguards challenges (e.g., MSR with protactinium separation leading to separation of ${ }^{233} \mathrm{U}$ ), hence not limiting or biasing any conclusions drawn. The timescales for deployment of any given thorium fuel cycle were also a key consideration in selecting a subset of leading thorium fuel cycles warranting further assessment.

The DOE-NE Evaluation and Screening Study [20] is the most comprehensive domestic or international study completed on all fuel cycle options, and it identifies 15 thorium fuel cycles options. Combining the findings of that assessment, along with the latest international developments identified in a literature review using key resources from the IAEA, Organization for Economic Cooperation and Development Nuclear Energy Agency, World Nuclear News, American Nuclear Society conferences, and others, the most significant thorium fuel cycle options were proposed to DNN R\&D for review and approval in an FY18 briefing prior to the detailed analysis and evaluations being completed. This step ensured that the fuel cycles identified align with DNN R\&D's insight in domestic and international developments.

The final criteria used to select the leading thorium fuel cycles from the candidate fuel cycles are shown below. Those further along in development and those most challenging to safeguard were considered.

- Commercial viability: The thorium fuel cycles were assessed based on whether they were commercially viable and their likelihood of implementation or commercial deployment.

- Active research: The active research conducted into each thorium fuel cycle was assessed based on the level of research: fundamental R\&D (theoretical); applied R\&D (fuel development and irradiation, test reactors); and deployment (demonstration reactors).

- National priorities or drivers (i.e., strategic value to country): The thorium fuel cycles were assessed based on national priorities including resource utilization, waste minimization, sustainability, and economics.

- Safeguards concerns or "safeguardability": The thorium fuel cycles were assessed based on potential safeguards challenges including new fuel forms, new signatures, and complex fuel cycles.

The output of this work is a shortlist of leading candidate thorium fuels, reactors, and fuel cycles, determined to be the most relevant for this scoping study. The following section summarizes the fuel cycle options; the key stages of each of the thorium fuel cycles used for the full evaluation, including flow diagrams; and the nuclear material present at each identified stage of the fuel cycles. The different evolutions of thorium implementation [e.g., LWRs (Norway), CANDU (CANada Deuterium Uranium) reactors (India), and MSRs (China, USA, USA-Indonesia)] are proposed as leading candidates. 


\subsection{Three Leading Candidate Thorium Fuel Cycles}

This section describes the three leading candidate thorium fuel cycles and the justification for their selection based on the selection criteria previously defined. Table 1 summarizes the leading thorium fuel cycles. The different evolutions of thorium implementation were chosen as leading fuel cycles representing four reactor types including: LWRs (e.g., Norway, USA), PHWRs (e.g., India), fast reactors (e.g., India), and MSRs (e.g., China, USA, USA-Indonesia).

Table 1. Leading thorium fuel cycles.

\begin{tabular}{|l|c|c|}
\multicolumn{1}{|c|}{ Fuel cycle } & \multicolumn{2}{c|}{ Reuntry/developer } \\
\hline $\begin{array}{l}\text { Multistage fuel cycle- } \\
\text { continuous recycle of }{ }^{233} \mathrm{U}\end{array}$ & India & $\begin{array}{l}\text { Heavy water reactor (Stage 1), sodium- } \\
\text { cooled fast breeder reactor (Stage 2), } \\
\text { advanced heavy water reactor (Stage 3) }\end{array}$ \\
\hline $\begin{array}{l}\text { Once-through or } \\
\text { continuous recycle in } \\
\text { pressurized water reactor }\end{array}$ & Norway (ThorEnergy), USA (WASB) & Pressurized water reactor \\
\hline $\begin{array}{l}\text { Continuous recycle of }{ }^{233} \mathrm{U} \\
\text { in MSR }\end{array}$ & $\begin{array}{c}\text { USA (Flibe Energy), USA-Indonesia } \\
\text { (ThorCon), China (thorium molten salt } \\
\text { reactor [TMSR]) }\end{array}$ & MSR (thermal fuel salt) \\
\hline
\end{tabular}

Although a number of these technologies are being developed by nuclear-weapon States (NWSs), any of these technologies could be commercially sold and exported to NNWSs. If operated in a State with a comprehensive safeguards agreement (CSA), the IAEA would be obligated to verify the peaceful use of that facility and all associated nuclear material. Additionally, some States (e.g., India) have legally binding safeguards agreements with the IAEA, referred to as item-specific safeguards agreements, rather than a CSA. The United States has previously included stipulations that all U.S.-origin civilian nuclear facilities and nuclear material are under safeguards in 123 Agreements for Peaceful Cooperation (named for Section 123 of the US Atomic Energy Act) in States without a CSA (e.g., the U.S.-India Nuclear Deal). Additionally, China, the United States, and the other NWSs party to the NPT have legally binding Voluntary Offer Agreements with the IAEA. This allows the IAEA to implement safeguards on selected facilities in NWSs. The IAEA sometimes selects facilities in NWSs that are unique so it can develop safeguards approaches or tools and technologies that will enable implementation of effective and efficient safeguards at such facilities. For example, the IAEA could select a Th/233U-fueled MSR in the United States or China to develop safeguards implementation procedures even if there were not yet any facilities of this type operating in an NNWS.

\subsubsection{Justification}

As discussed in Section 1.1, the three leading fuel cycles were evaluated on four criteria: commercial viability, active research, national priorities, and safeguards concerns. Table 2 presents the basis for the selection of the three leading thorium fuel cycles using these criteria. 
Table 2. Basis for the selection of the three leading thorium fuel cycles referencing specific country developments as examples.

\begin{tabular}{|c|c|c|c|}
\hline $\begin{array}{l}\text { Chosen } \\
\text { thorium fuel } \\
\text { cycle }\end{array}$ & $\begin{array}{l}\text { Multistage fuel cycle, resulting } \\
\text { in continuous recycle of }{ }^{233} \mathrm{U} \\
\text { Stage 1: Generates Pu for Stage } \\
2 \\
\text { Stage 2: Breeds }{ }^{233} \mathrm{U} \text { and } \mathrm{Pu} \\
\text { Stage 3: }{ }^{233} \mathrm{U} \text { continuous recycle } \\
\text { (e.g, India) }\end{array}$ & $\begin{array}{l}\text { Once-through or } \\
\text { continuous recycle in } \\
\text { pressurized water reactor } \\
\text { (PWR) } \\
\text { [e.g., Norway (ThorEnergy), } \\
\text { U.S. (WASB)] }\end{array}$ & $\begin{array}{l}\text { Continuous recycle of }{ }^{233} \mathrm{U} \\
\text { in MSR } \\
\text { [e.g., U.S. (FLiBe), China } \\
\text { (TMSR), U.S.-Indonesia } \\
\text { (ThorCon)] }\end{array}$ \\
\hline $\begin{array}{l}\text { 1. Commercial } \\
\text { viability }\end{array}$ & $\begin{array}{l}\text { Fuel cycle concept: Yes, } \\
\text { commercially viable. However, } \\
\text { this appears to be a complex fuel } \\
\text { cycle to achieve continuous } \\
\text { recycle. }\end{array}$ & $\begin{array}{l}\text { Fuel cycle concept: Yes, } \\
\text { commercially viable. } \\
\text { Extensive industry interest } \\
\text { from fuel vendors. Majority } \\
\text { of required technology } \\
\text { already commercially } \\
\text { demonstrated. }\end{array}$ & $\begin{array}{l}\text { Fuel cycle concept: } \\
\text { Commercial viability is } \\
\text { uncertain because of relative } \\
\text { immaturity of concepts. } \\
\text { However, shows extensive } \\
\text { potential, driving interest } \\
\text { and investment. }\end{array}$ \\
\hline $\begin{array}{l}\text { 2. Active } \\
\text { research }\end{array}$ & $\begin{array}{l}\text { India: Heavy water reactors } \\
\text { (HWRs) and fast breeder reactors } \\
\text { beyond demonstration phases } \\
\text { and approaching commercial } \\
\text { deployment. All associated fuel } \\
\text { cycle infrastructure also at } \\
\text { demonstration level. Advanced } \\
\text { HWR at applied research level. }\end{array}$ & \begin{tabular}{|l|} 
Norway: Irradiation \\
programs were undertaken \\
and have concluded in \\
Halden. Fuel development in \\
conjunction with the United \\
States, France, and UK. \\
-U.S.: Extensive analysis \\
completed in the past; no \\
current research under way.
\end{tabular} & $\begin{array}{l}\text { China: Demonstration reactor } \\
\text { under construction. } \\
\text { U.S.: Flibe Energy, Inc. startup } \\
\text { company received } \sim 2.5 \mathrm{M} \\
\text { from DOE-NE. ThorCon (USA- } \\
\text { Indonesia): Startup company } \\
\text { received few hundred \$K } \\
\text { from DOE-NE with focus on } \\
\text { Indonesia deployment. }\end{array}$ \\
\hline $\begin{array}{r}\text { 3. National } \\
\text { priorities }\end{array}$ & $\begin{array}{l}\text { India: Large thorium reserves. } \\
\text { Thorium fuel cycle being } \\
\text { developed to meet energy } \\
\text { demands. Potential military } \\
\text { application. }\end{array}$ & $\begin{array}{l}\text { Norway: Large thorium } \\
\text { reserves being actively } \\
\text { pursued by Norwegian } \\
\text { government (ThorEnergy); } \\
\text { additive for } \mathrm{UO}_{2} \text { PWR fuel to } \\
\text { improve economics; and } \\
\text { potential Pu management } \\
\text { option. } \\
\text { U.S.: not a national priority, } \\
\text { but historic interest in } \\
\text { exploring thorium use in } \\
\text { LWRs (e.g., Shippingport } \\
\text { PWR, Indian Point I PWR, Elk } \\
\text { River boiling water reactor). }\end{array}$ & $\begin{array}{l}\text { China: extensive nuclear } \\
\text { growth program and export } \\
\text { market (resource utilization } \\
\text { and waste minimization). } \\
\text { U.S.: Flibe: none. } \\
\text { ThorCon: focused on export } \\
\text { to Indonesia. }\end{array}$ \\
\hline $\begin{array}{l}\text { 4. Safeguards } \\
\text { concerns }\end{array}$ & $\begin{array}{l}\text { Full separations of }{ }^{233} \mathrm{U} \text { and } \mathrm{Pu} \text {. } \\
\text { Complex fuel cycle within the } \\
\text { country. }\end{array}$ & $\begin{array}{l}\text { Fuel designs that include } \\
\text { driver and blanket regions. }\end{array}$ & $\begin{array}{l}\text { Liquid fuel, various forms of } \\
\text { separation with }{ }^{233} \mathrm{U} \text { stream. }\end{array}$ \\
\hline
\end{tabular}

\subsubsection{Fuel Cycle 1: Multistage Thorium Fuel Cycle}

India's three-stage nuclear power program serves as an example of a multistage approach to a thorium fuel cycle, which is shown in Figure 1 [21-24]. At present (Stage 1), PHWRs that burn natural uranium produce depleted uranium (DU) as well as plutonium. In 10 years (Stage 2), the PHWR outputs are planned to operate fast breeder reactors (FBRs), which will convert natural uranium into 
plutonium more efficiently than PHWRs. In several decades, Stage 3 will involve operating advanced heavy water reactors (AHWRs) that will operate initially with thorium, plutonium, and DU mixed-oxide (MOX) fuel. Irradiated fuel from an AHWR can be reprocessed to extract ${ }^{233} \mathrm{U}$. After reprocessing becomes sustainable, AHWRs will transition to using recycled ${ }^{233} \mathrm{U}$ in place of DU. Therefore, in this multistage approach, ${ }^{233} \mathrm{U}$ is present at the back end of Stage 2 and in Stage 3. Plutonium is present in all stages. This multistage thorium fuel cycle was modeled for this scoping study. The reactor physics models are described in Sections 2.3.2 and 2.3.3, and the calculated nuclear material inventories are provided.

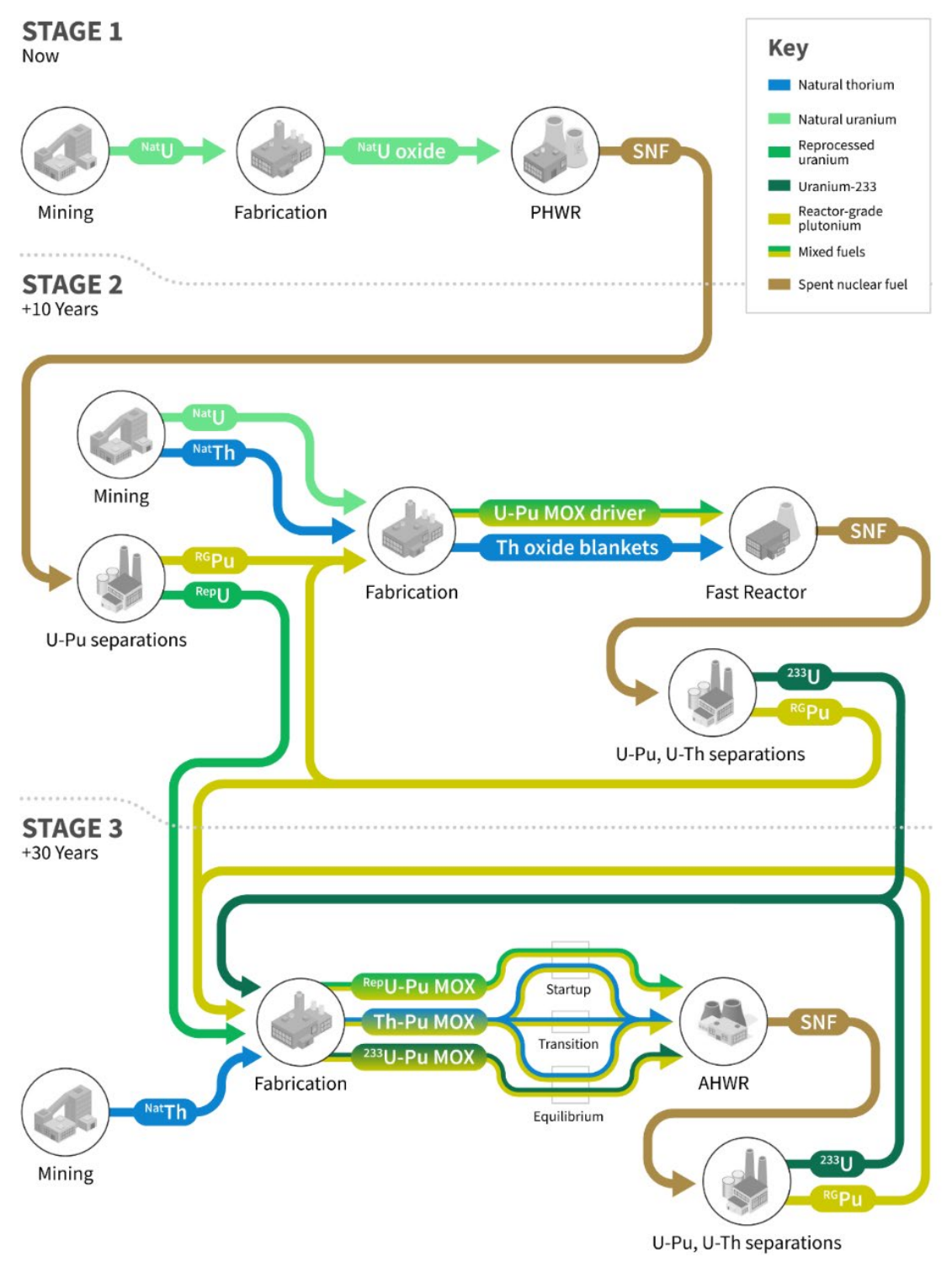

Figure 1. Multistage thorium fuel cycle.

\subsubsection{Fuel Cycle 2: Once-through Thorium Fuel Cycle in a PWR}

Figure 2 illustrates a once-through approach to a thorium fuel cycle. Operation of pressurized water reactors (PWRs) fueled by LEU fuel leads to the production of DU fuel, as well as plutonium. However, the proposed once-though thorium cycle would exchange a portion of the fertile matrix of ${ }^{238} \mathrm{U}$ in LEU 
fuel with thorium [25]. Thus, Stage 1 would use thorium and uranium oxide fuel to power the PWRs. Any bred plutonium from the Stage 1 irradiated fuel would be extracted and combined with thorium to create fuel for Stage 2 PWRs. Stage 2 reactors feature improved plutonium utilization and an enhanced breeding ratio. For the once-through design, the irradiated Stage 2 fuel moves to disposal. Alternatively, the Stage 2 products could be reprocessed to extract unused isotopes and fabricated into new fuel. This LWR-based thorium fuel cycle was modeled for this scoping study. The reactor physics models are described in Section 2.3.1, and the calculated nuclear material inventories are also provided. This fuel cycle is analogous to current LWRs operating on a U/Pu fuel cycle and, therefore, serves as a baseline for comparison of current safeguards technology implementation against future needs.

STAGE 1

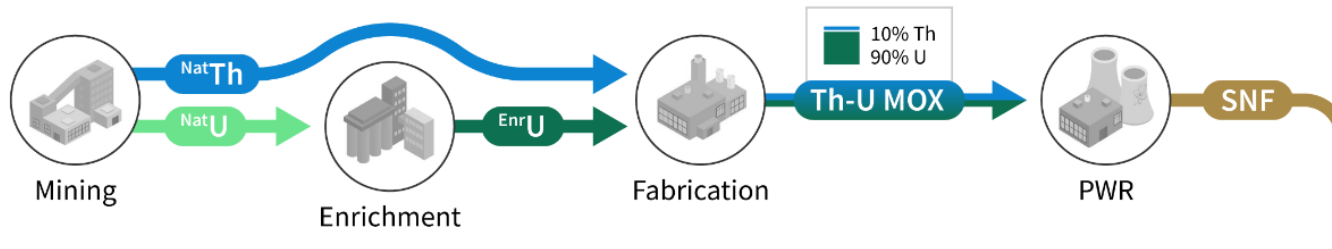

STAGE 2

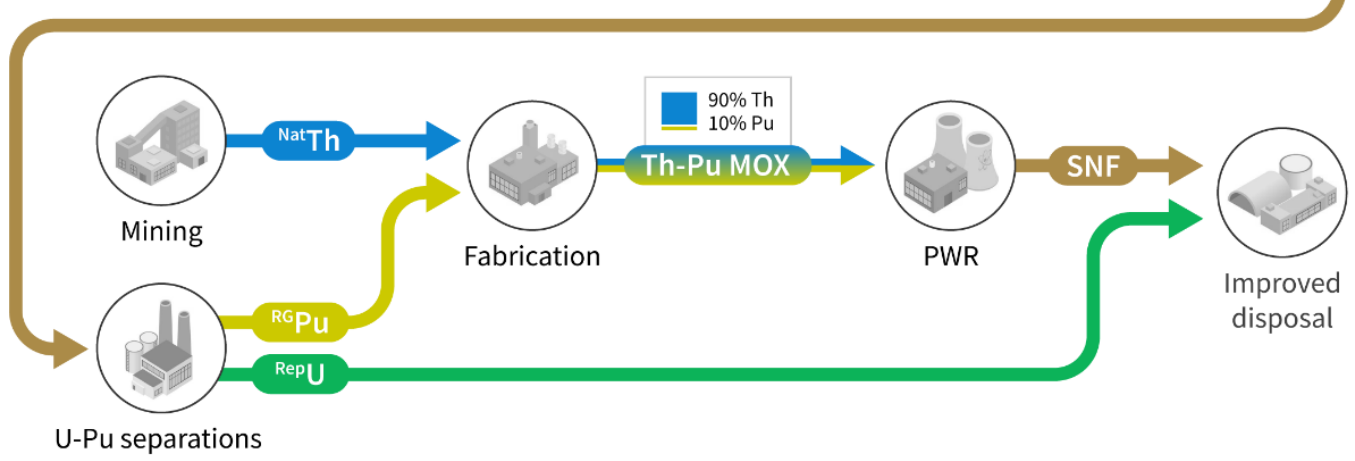

$$
\begin{array}{|l|lll}
\hline \text { Key } & \text { Natural thorium } & \text { Natural uranium } & \text { Reprocessed uranium } \\
\text { Reactor-grade plutonium } & \text { Mixed fuels } & \text { Spent nuclear fuel }
\end{array}
$$

Figure 2. Once-through thorium fuel cycle in a PWR.

\subsubsection{Fuel Cycle 3: Continuous-recycle Thorium Fuel Cycle in an MSR}

Figure 3 shows a continuous-recycle thorium fuel cycle in an MSR. Unlike conventional reactors that use solid fuel, typically in the form of a metal oxide, the MSR utilizes uranium and thorium salts (typically fluorides or chlorides) mixed with other salts (e.g., LiF, $\mathrm{NaF}, \mathrm{NaCl}, \mathrm{BeF}_{2}$ ) as the fuel. These reactors can operate in continuous-cycle mode, where the irradiated fuel is reprocessed continuously to refresh the fuel salt for the next cycle. FLiBe, a mixture of lithium fluoride and beryllium fluoride, is a common salt mixture used in continuous-recycle thorium fuel cycles. This salt mixture is used in Flibe Energy's liquid fluoride thorium reactor (LFTR) design [26] as well as China's TMSR design [27]. Several other salts are potential candidates for MSRs such as the sodium fluoride and beryllium fluoride salt mixture proposed by ThorCon [28]; however, we decided to pursue FLiBe because of DOE's investment and other project interests in Flibe Energy's LFTR design. An MSR utilizing thorium 
can start the reactor either using a two-fluid design (a fuel salt, including LEU fluoride, and a blanket salt that includes thorium fluoride) or a single-fluid design (no blanket salt, thus the fuel salt contains both uranium fluoride and thorium fluoride). The output from the reactor includes protactinium, which is generated by thorium conversion. The protactinium and thorium are extracted from the outlet stream(s) to generate additional ${ }^{233} \mathrm{U}$ and thorium fluorides that are fed back into the reactor. The MSR thorium fuel cycle, using a single-fluid MSR design, was modeled for this scoping study. The reactor physics model is described in Section 2.3.4, and the calculated nuclear material inventories are provided.

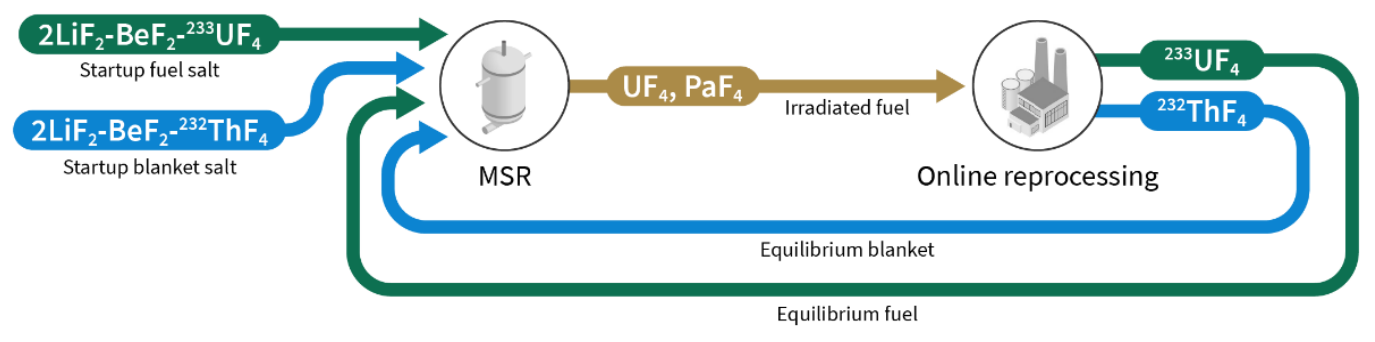

Figure 3. Continuous-recycle thorium fuel cycle in an MSR.

\subsection{Key Observations Affecting Implementation of Safeguards Technology}

\subsubsection{Varying Isotopic Mixtures, Fuel Forms, and Enrichment Levels}

From these three leading fuel cycles, it is possible to ascertain the types of items and material compositions bearing Th and/or ${ }^{233} \mathrm{U}$ (as well as plutonium) that would be subject to international nuclear safeguards and require verification. Some examples are listed below. Current verification measurement capabilities to safeguard nuclear material are described in Section 3 and are evaluated against examples of these isotopic mixtures, fuel forms, and enrichment levels.

- Multistage thorium fuel cycle ${ }^{1}$

O Stage 1 (HWR): ${ }^{\text {nat } U ~ f u e l ~}$

- Stage 2 (FBR): U-Pu MOX driver fuel, Th oxide blanket

- Stage 3 (AHWR): Th-Pu MOX, ${ }^{233} \mathrm{U}-\mathrm{Pu}$ MOX fuel option

- Once-through thorium fuel cycle in a PWR

Stage 1: Th-U MOX fuel

○ Stage 2: Th-Pu MOX fuel

\footnotetext{
${ }^{1}$ Note that we are discussing this multistage thorium fuel cycle option generically in terms of safeguards needs, not necessarily how it is currently implemented in India.
} 
- Continuous-recycle thorium fuel cycle in an MSR

- Potential for two-fluid (separate fuel and blanket salts) and single-fluid designs for saltfueled MSR concepts

$\circ$ FLiBe (or other) salt with ${ }^{233} \mathrm{U}$ (breeding ratio $<1$ ) and/or ${ }^{232} \mathrm{Th}$ (breeding ratio $>1$ )

- Could also be supplemented with other fissile isotopes such as ${ }^{235} \mathrm{U}$ and ${ }^{239} \mathrm{Pu}$

$0 \quad{ }^{233} \mathrm{~Pa}$ and ${ }^{233} \mathrm{U}$ in-growth from ${ }^{232} \mathrm{Th}$ in thorium-bearing salt

- Online salt reprocessing that involves several chemical forms of heavy metals

These findings show that $\mathrm{Th}$ and ${ }^{233} \mathrm{U}$ are usually present with other nuclear materials (e.g., $\mathrm{Pu},{ }^{235} \mathrm{U}$ ). Therefore, from a safeguards measurement perspective, there is the potential to verify not only ${ }^{233} \mathrm{U}$ mass, but also the mass of multiple fissile isotopes present in a single fuel assembly. Furthermore, a range of ${ }^{235} \mathrm{U}$ enrichment levels may be present in Th-bearing fuel. Th-bearing fuel for use in a thermal spectrum LWR can be highly enriched in ${ }^{235} \mathrm{U}$ because the thermal capture cross-section of ${ }^{232} \mathrm{Th}$ is about three times larger than ${ }^{238} \mathrm{U}$, and a much higher fissile enrichment is required for ${ }^{232} \mathrm{Th}$-fueled cores in thermal spectrum reactors. Therefore, another significant observation is that these leading thorium fuel cycles may also require HEU verification.

\subsubsection{Other Technical Factors Affecting Implementation of Safeguards Technology}

Technical factors for thorium fuel cycles that lead to differences in the implementation of safeguards technology, in addition to those summarized in Section 1.3.1, include the following:

- Different fuel designs (e.g., fuel physical and chemical form, isotopic content, geometry, mechanical design)

- Different reprocessing and separation schemes (e.g., continuous recycle, online continuous recycle)

- Different fuel cycle infrastructure likely required within a State to have a thorium fuel cycle

- Different reactor designs

- Variations in reactor operations (e.g., startup, shutdown, and reactivity transients)

The dose in the proximity of thorium fuel cycle facilities is expected to be substantially greater than that of conventional fuel cycles, impacting sensor detection limits and lifetimes, accessibility for safeguards inspectors, and operations.

Today, safeguards technology is heavily reliant on nuclear material accountancy, and nondestructive assay (NDA) is the workhorse of that approach. Item counting or sample assay may not be possible for bulk nuclear material in some thorium fuel cycles, such as the MSR thorium cycle, where fuel is in a molten form. As a result, alternative or additional measures may be sought (e.g., greater reliance on 
destructive assay [DA] including both online and environmental sampling, or design information verification); the frequency or intensity of inspections may also be increased. Where items are present (e.g., discrete fuel bundles), differences in safeguards measures and activities may exist.

\subsubsection{Safeguards Technology R\&D Timeline: Safeguards Technology Development Timeline Compared with the Timeline to Commercial Implementation of Thorium Fuel Cycles}

Figure 4 illustrates an MSR program as an example timeline to commercial implementation of a leading thorium fuel cycle. Based on China's planned thorium fuel cycle using a TMSR, this example illustrates the potential timelines for commercial implementation of thorium fuel cycles and the development stages involved. Figure 4 shows near-term development activities including experimental verification of a Th/U fuel cycle, which can be expected to take place until around 2025. Mid-term development activities will feature commercial demonstration of a fluoride-salt-cooled high-temperature reactor concept. Long-term activities are planned for around 2030, which include full Th/U fuel cycle demonstration. In the case of the Chinese program, demonstration will be performed using a liquid-fueled thorium molten salt reactor (TMSR-LF) demonstration reactor.

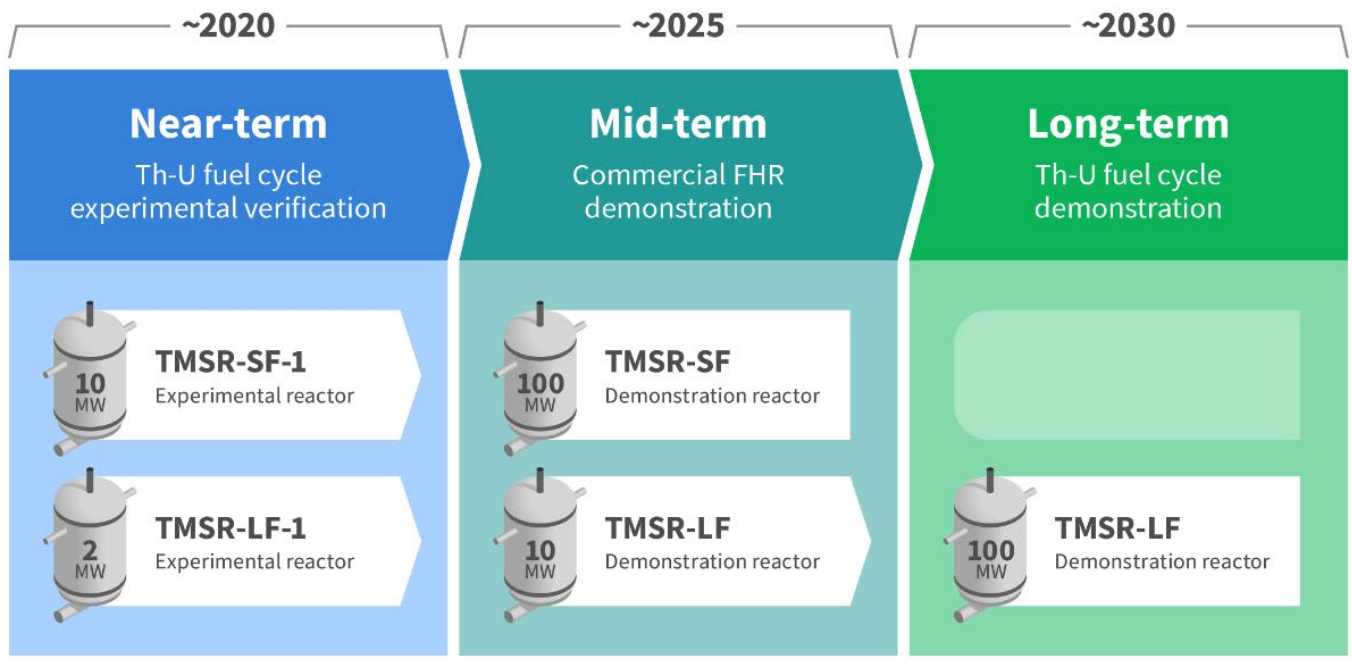

Figure 4. Sample timeline to commercial implementation of a leading thorium fuel cycle.

Figure 5 provides a sample safeguards technology R\&D timeline (top panel) and development timeline (bottom panel). The top panel references the sample timeline to commercial implementation of the leading thorium fuel cycle shown in Figure 4. As illustrated in Figure 5, the timeline for any new safeguards technology development is $\sim 10$ years from lowest technology readiness level (TRL) (i.e., concept) to highest TRL (i.e., commercial adoption and field implementation) based on the need for the development stages outlined. Therefore, planning for new fuel cycle and reactor technology developments in advance and determining modifications to existing instrumentation in advancerather than having to develop new instrumentation-moves safeguards verification technology further along the technology development lifecycle and implementation timeline, somewhat favoring the adoption and modification of existing technology and commercial-off-the-shelf (COTS) offerings. Using the example safeguards technology development timeline in Figure 5, a new technology would require initial requirements scoping and basic $R \& D$ ( $\sim 2$ years), a design phase ( 1 year), prototyping ( 1 year), and laboratory testing of that prototype ( 1 year) prior to the technology being considered 
for a representative field trial. A field trial might take place in several experimental campaigns over $\sim 1$ year prior to acceptance for use or commercialization ( $\sim 1$ additional year). Finally, IAEA approval for use and training might be expected to follow an iterative process with the technology developers, resulting in a final phase of $\sim 3$ years, prior to adopting the technology for in-field verification activities. Any early identification of COTS technologies would eliminate the need for the first three phases and move a technology to laboratory testing, thus shortening the technology development timeline.

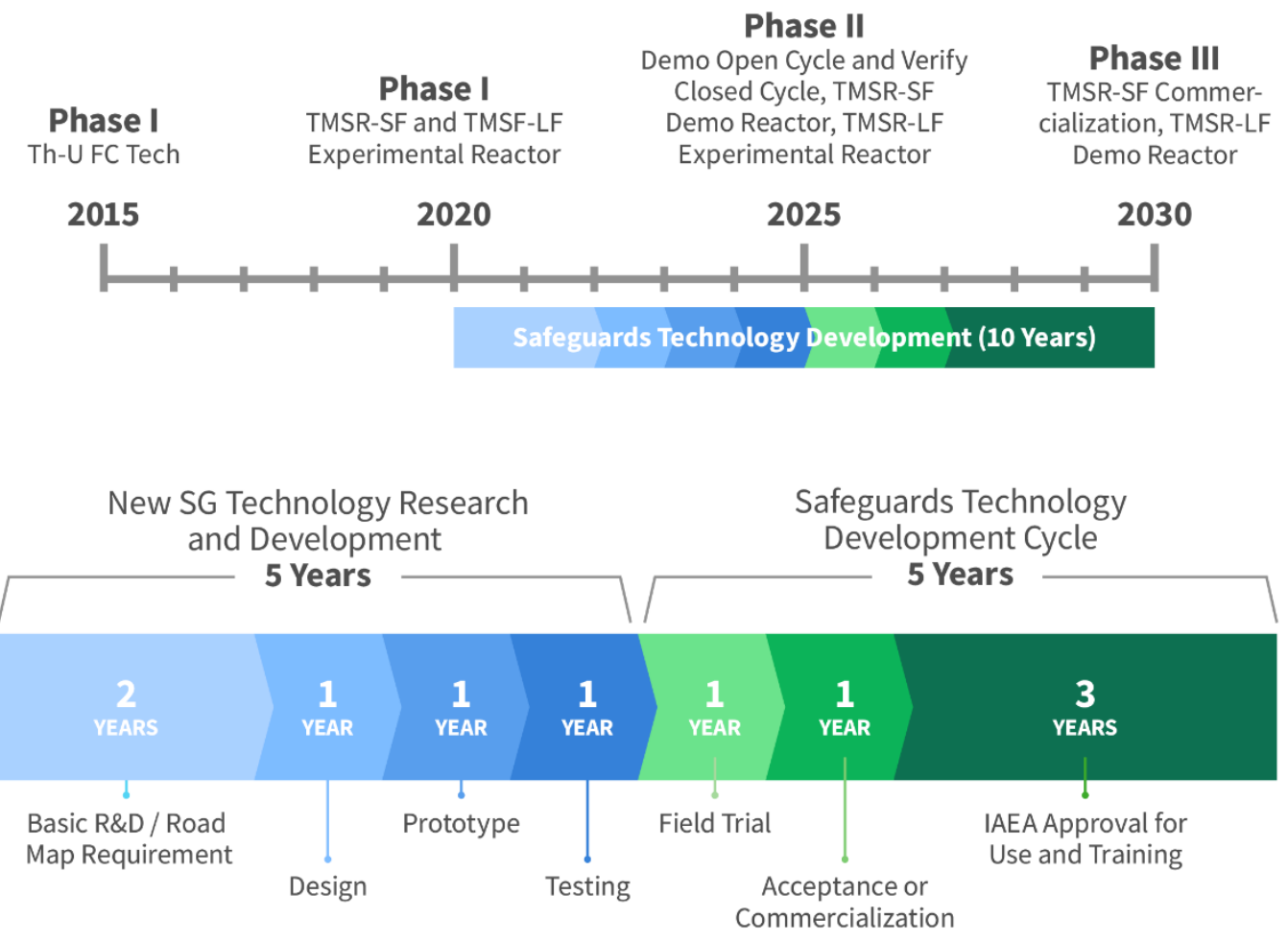

Figure 5. Example thorium fuel cycle implementation timeline (top) vs. example safeguards technology development timeline ${ }^{2}$ (bottom).

\footnotetext{
${ }^{2}$ Time durations for each phase of safeguards technology development are approximations only and are provided for illustration. These estimates are based on conversations with various safeguards technology developers with DOE national laboratory and commercial sector experience and do not reference a specific technology or IAEA protocol.
} 


\section{Fuel Cycle and Reactor Simulations}

This section describes the fuel cycle and reactor simulations that were performed to generate the nuclear material inventories and select radiation signatures for the leading thorium fuel cycles described in Section 1. The objective for this section is presented in Section 2.1, and the method for simulating nuclear material inventories is discussed in Section 2.2. Section 2.3 presents the results of modeling nuclear material inventories for each of the selected thorium fuel cycles. Section 2.4 provides results for simulating spent fuel dose rates, and key safeguards observations are discussed in Section 2.5.

\subsection{Objective of Fuel Cycle and Reactor Simulations}

Inventory calculations were performed to determine both the fresh and spent fuel nuclear material compositions that would need to be safeguarded and verified at each fuel cycle stage as well as the resulting radiation signatures that would need to be measured to verify that nuclear material inventory. In turn, this enables a determination of the measurement modalities that are needed and any gaps in current technologies with respect to meeting the identified measurement needs. The radiation dose was also calculated at both contact and standoff distances for select spent nuclear fuel (SNF) cases to consider the effect of dose on future instrumentation requirements. Measurement capabilities and gaps are discussed in Section 3.

\subsection{Simulation Method for Inventory Calculations}

Fuel cycle and reactor models were produced to calculate the nuclear material inventories for select thorium fuel cycles. Using Oak Ridge National Laboratory's (ORNL's) SCALE (Standardized Computer Analyses for Licensing Evaluation) code package [9], depletion calculations were run for each thorium fuel cycle to produce inventory and associated source terms. The reactor depletion and inventory tools under the SCALE code suite are well validated for fuel inventory and source term calculations and are used by more than 7,000 users worldwide, including the US Nuclear Regulatory Commission (NRC). These tools have been developed specifically for reactor analysis and inventory calculations, including $\mathrm{U}, \mathrm{Pu}$, and Th fuels, and for all leading reactor types including but not limited to LWRs, CANDU reactors, and MSRs.

Reactor simulations were completed based on fuel and reactor design information available to ORNL through previous programs. Spent fuel inventories and associated source terms were produced to reflect the stages of the leading thorium fuel cycles corresponding to reactor operations. The ORIGEN (Oak Ridge Isotope Generation and Depletion) code was used to produce gamma spectra for the key stages of the fuel cycle (e.g., spent fuel, feed fissile material). A similar analysis was produced for LWR $\mathrm{UO}_{2}$ and LWR MOX fuel, and the spectra formed the basis for comparison against the thorium fuel cycle signatures for the cases described in Section 2.3. Similar, unique signatures were identified at this stage to begin to inform the potential applicability of existing safeguards instruments and systems.

The ORIGEN code was used to predict the nuclide compositions due to transmutation under irradiation and decay; continuous or batch chemical/physical processing would be a consideration for 
future work. After the ORIGEN code calculated the isotopic concentration at each step, the ORIGEN gamma yield libraries were used to calculate the intensity of all gamma emission lines produced by these isotopes. Next, these gamma lines were provided to the Sandia National Laboratoriesdeveloped GADRAS (Gamma Detector Response and Analysis Software) code to simulate the expected detector response functions.

\subsection{Simulated Thorium Fuel Cycle and Reactor Cases}

Reactor libraries parameterized by initial enrichment and thorium content have been created for LWR fuels using both homogeneous and heterogeneous (i.e., seed-and-blanket) designs. These libraries can be used together to compute material flows and compositions for once-though, multi-stage, and continuous-recycle thorium fuel cycles during transition and at equilibrium. For the HWR and FBR models, proposed thorium-bearing fuel designs were simulated and can be used as general archetypes for fuel cycles containing these reactor types. The results of these simulations are isotopic inventories during burnup and decay. Using these isotopic inventories, follow-on simulations have been performed that emulate gamma spectrometer response due to spent fuel measurements (see Section 3.5.1.2). These spectra are representative of postirradiation safeguards measurements.

The following sections present isotopic inventories from models representing each of the three leading thorium fuel cycles discussed in Section 1. The once-through thorium fuel cycle from Section 1.2.3 is represented using traditional PWR designs as discussed in Section 2.3.1, where a parametric study is used to evaluate the impact of thorium content and uranium enrichment. The multistage thorium fuel cycle from Section 1.2.2 encompasses multiple reactor types and is represented by the HWR and FBR models discussed in Sections 2.3.2 and 2.3.3, respectively. A parametric study is also used to evaluate the HWR designs, while a case study is used to evaluate the FBR. Finally, the continuous-recycle thorium fuel cycle from Section 1.2.4 is represented by the MSR model discussed in Section 2.3.4 using a case study. Parametric studies in the case of the FBR and MSR models were undesirable due to the large variability between potential designs.

Table 3 shows the reactor implementation of these leading thorium fuel cycles and representative fuel forms chosen for modeling. Detailed isotopic compositions of these fuel forms are listed in Tables 4-28 in Section 2 of this report. The rightmost column of Table 3 provides a cross-reference to the Section 2 tables where those specific isotopic compositions may be found. Note that other fuel forms are possible (e.g., carbide or metal fuel forms, FLiNaK salt) but were not modeled. 
Table 3. Reactor implementation and representative fuel forms for chosen thorium fuel cycles. Choice depends on reactor design.

\begin{tabular}{|c|c|c|c|}
\hline Chosen thorium fuel cycle & Reactor & Representative fuel forms & $\begin{array}{c}\text { Table(s) for } \\
\text { fuel } \\
\text { composition }\end{array}$ \\
\hline $\begin{array}{l}\text { Once-through or continuous } \\
\text { recycle in PWR } \\
\text { [e.g., ThorEnergy (Norway), } \\
\text { WASB (US)] }\end{array}$ & PWR & $\mathrm{UO}_{2}$ and $\mathrm{UO}_{2}+\mathrm{ThO}_{2}$ pellets & Tables 4-13 \\
\hline \multirow{3}{*}{$\begin{array}{l}\text { Multistage fuel cycle, resulting in } \\
\text { continuous recycle of }{ }^{233} \mathrm{U} \\
\text { Stage 1: Generates Pu for Stage } 2 \\
\text { Stage 2: Breeds }{ }^{233} \mathrm{U} \text { and } \mathrm{Pu} \\
\text { Stage 3: }{ }^{233} \mathrm{U} \text { continuous recycle } \\
\text { (e.g., India) }\end{array}$} & HWR (Stage 1) & ${ }^{\text {nat }} \mathrm{UO}_{2}$ pellets & Table 14 \\
\hline & FBR (Stage 2) & $\begin{array}{l}\mathrm{ThO}_{2} \text { pellets (blankets) } \\
\mathrm{UO}_{2}+\mathrm{PuO}_{2} \text { pellets (driver) }\end{array}$ & Table 21 \\
\hline & AHWR (Stage 3) & $\begin{array}{l}\mathrm{ThO}_{2}+\mathrm{PuO}_{2} \text { pellets (startup) } \\
\mathrm{ThO}_{2}+{ }^{233} \mathrm{UO}_{2} \text { pellets (equilibrium) }\end{array}$ & Tables $15-18$ \\
\hline $\begin{array}{l}\text { Continuous recycle of }{ }^{23} \mathrm{U} \text { in MSR } \\
\text { [e.g., Flibe (US), TMSR (China), } \\
\text { ThorCon (US-Indonesia)] }\end{array}$ & $\begin{array}{l}\text { MSR (thermal } \\
\text { fuel salt) }\end{array}$ & $\begin{array}{l}\text { Th(FLiBe) fuel salt; blanket not } \\
\text { modeled for single fluid design }\end{array}$ & Table 22 \\
\hline
\end{tabular}

\subsubsection{Pressurized Light Water Reactor}

Three types of pressurized light water reactor (PWR) fuel were simulated. These models were based on a typical Westinghouse $17 \times 17$ fuel design, where $17 \times 17$ implies a square pitched grid of 17 pin locations in both the $x$ - and $y$-directions. Twenty-four of the pin locations are occupied by control rod guide tubes, and the central location is occupied by an instrument guide tube, leaving 264 fuel pin locations per assembly. This arrangement of pins is known as a fuel lattice, which are nearly always symmetric for PWRs. Section 2.3.1.1 presents the simulation results for initial use fuels, while Section 2.3.1.2 presents simulation results using recycled fuel.

\subsubsection{Initial Use PWR Thorium Fuels}

The initial PWR reactor simulations consider thorium fuels without any ${ }^{233} \mathrm{U}$ present initially. These fuels could be used in once-through fuel cycles or as the first generation of a recycle fuel cycle. When a single fuel material is used, the lattice is referred to as homogeneous. When multiple fuel materials are used, the lattice is referred to as heterogeneous. A heterogeneous lattice could consist of many different materials in symmetric pins, but these studies are limited to a single heterogeneous design. Three model templates were used for these studies:

- Baseline $\mathrm{UO}_{2}$ homogeneous PWR fuel lattice

- $\mathrm{UO}_{2}-\mathrm{ThO}_{2}$ homogeneous PWR fuel lattice

- $\mathrm{UO}_{2}-\mathrm{ThO}_{2}$ heterogeneous PWR fuel lattice

These PWR model templates were created for other projects and have been extended for this work $[16,29]$. 


\section{Baseline $\mathrm{UO}_{2}$ Homogeneous PWR Fuel Lattice}

Uranium-based fuel models were created as a basis of comparison to thorium-based fuels. These models use a homogeneous lattice consisting of a single enrichment (see Figure 6). Actual modern fuel design is optimized for use in reactors, and lattices typically contain multiple enrichments and burnable absorbers to flatten power peaking over the lifetime of the fuel. Although a single enrichment assembly with no burnable absorbers is increasingly less common in core design, these homogeneous enrichment lattices are sufficient for fuel cycle analysis that seeks to understand typical gross fuel mass flows and isotopic composition. In other words, these models are representative of average fuel use in a reactor; specific fuel designs would include some variations. Homogeneous PWR fuel lattices were modeled with $100 \% \mathrm{UO}_{2}$ with enrichment ranging from 1 to $20 \mathrm{wt} \%{ }^{235} \mathrm{U}$. Table 4 shows the bounding $\mathrm{UO}_{2}$ PWR fuel compositions (uranium vector) for the modeled LEU and HEU designs.

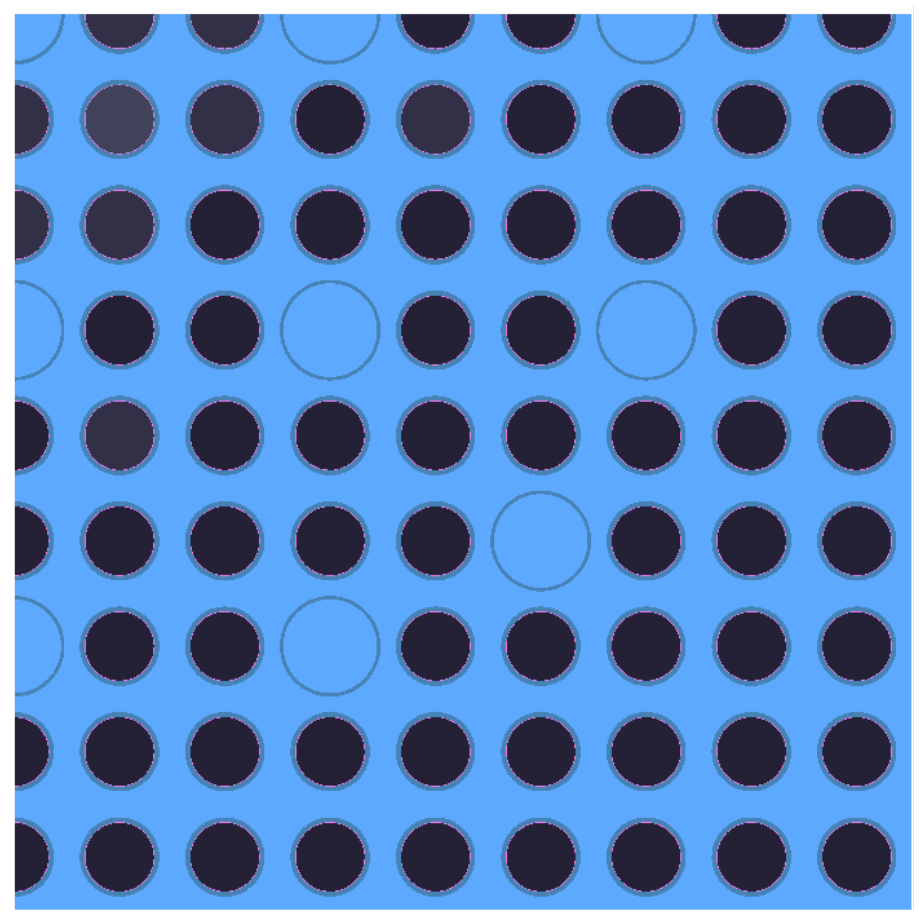

Figure 6. Homogeneous PWR quarter fuel lattice. $\mathrm{UO}_{2}$-fueled pins are shown in black. Light blue represents water (coolant/moderator). The top left corner is the lattice center (instrument tube location), and 8 of 24 control rod guide tubes are shown.

Table 4. Bounding $\mathrm{UO}_{2}$ PWR fuel compositions used as the baseline against which thorium-based PWR fuels are compared.

\begin{tabular}{|c|c|c|c|c|c|c|c|c|c|c|c|c|}
\hline \multirow{2}{*}{$\begin{array}{c}\text { Fuel } \\
\text { composition }\end{array}$} & \multicolumn{2}{|l|}{ Th } & \multicolumn{5}{|c|}{$\mathbf{U}$} & \multicolumn{5}{|c|}{$\mathbf{P u}$} \\
\hline & ${ }^{232} \mathrm{Th}$ & ${ }^{232} U$ & ${ }^{233} \mathrm{U}$ & ${ }^{234} \mathrm{U}$ & ${ }^{235} \mathrm{U}$ & ${ }^{236} \mathrm{U}$ & ${ }^{238} \mathrm{U}$ & ${ }^{238} \mathrm{Pu}$ & ${ }^{239} \mathrm{Pu}$ & ${ }^{240} \mathrm{Pu}$ & ${ }^{241} \mathrm{Pu}$ & ${ }^{242} \mathrm{Pu}$ \\
\hline \multirow[t]{2}{*}{ Low enriched } & $0.0 \%$ & \multicolumn{6}{|c|}{$100.0 \%$} & \multicolumn{5}{|c|}{$0 \%$} \\
\hline & $0.0 \%$ & $0.0 \%$ & $0.0 \%$ & $0.077 \%$ & $1.0 \%$ & $0.0046 \%$ & 98.9877\% & $0 \%$ & $0 \%$ & $0 \%$ & $0 \%$ & $0 \%$ \\
\hline \multirow{2}{*}{$\begin{array}{l}\text { High } \\
\text { enriched }\end{array}$} & $0.0 \%$ & \multicolumn{6}{|c|}{$100.0 \%$} & \multicolumn{5}{|c|}{$0 \%$} \\
\hline & $0.0 \%$ & $0.0 \%$ & $0.0 \%$ & $0.1987 \%$ & $20.0 \%$ & $0.0920 \%$ & $79.7093 \%$ & $0 \%$ & $0 \%$ & $0 \%$ & $0 \%$ & $0 \%$ \\
\hline
\end{tabular}


Figure 7 shows the resulting thorium, uranium, and plutonium mass concentrations vs. effective full power days (EFPDs) for the homogeneous PWR fuel lattice composed of $100 \% \mathrm{UO}_{2}$ at a range of 1$20 \mathrm{wt} \%{ }^{235} \mathrm{U}$ enrichment. These mass concentrations are expressed as kilograms per megagram of initial heavy metal. The shaded areas represent the mass concentrations over the range of initial enrichments between the two bounding enrichment conditions. Section 2.3.5 presents a comparison of ${ }^{233} \mathrm{U}$ and ${ }^{232} \mathrm{U}$ inventories across reactor types.

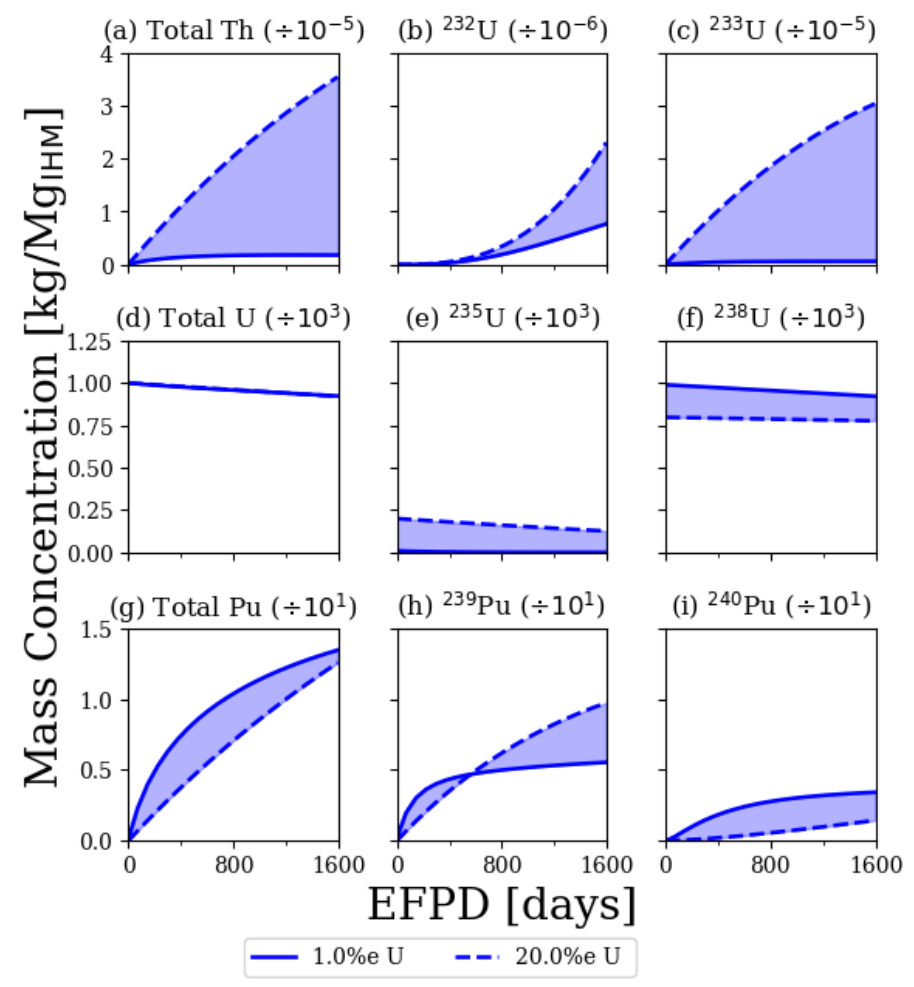

Figure 7. Th, U, Pu, mass concentrations vs. EFPDs for the homogeneous PWR fuel lattice composed of $100 \% \mathrm{UO}_{2}$ enriched from 1 to $20 \%$.

\section{$\mathrm{UO}_{2}-\mathrm{ThO}_{2}$ Homogeneous PWR Fuel Lattice}

From the perspective of fuel design, perhaps the simplest way to use thorium is to employ a homogenous mixture of both uranium oxide and thorium oxides (U-Th MOX) in all pins in the assembly. In contrast to a single oxide homogeneous lattice, a MOX lattice can be parameterized, both by enrichment and MOX fraction. For example, a hypothetical homogeneous U-Th MOX might consist of $50 \%$ thorium and $50 \%$ uranium enriched to $15 \mathrm{wt} \%{ }^{235} \mathrm{U}$, while another hypothetical mixture might consist of $75 \%$ thorium and $25 \%$ uranium at $19.9 \mathrm{wt} \%{ }^{235} \mathrm{U}$ [20]. Note that $26 \% \mathrm{UO}_{2}$ to $74 \% \mathrm{ThO}_{2}$ (where the uranium is enriched to $\left.20 \mathrm{wt} \%{ }^{235} \mathrm{U}\right)$ is roughly equivalent to $100 \% \mathrm{UO}_{2}\left(4.0 \mathrm{wt} \%{ }^{235} \mathrm{U}\right)$ in terms of a single assembly lifetime averaged reactivity. The MOX fraction and enrichment for a particular fuel design would depend on several factors, including steady-state and transient fuel performance limitations, overall core design of reactor, economics of enrichment, and the fuel cycle pathway after the fuel is removed from the reactor (reprocessing vs. disposal). Because ${ }^{232}$ Th itself is not fissile, mixing natural thorium oxide with any uranium oxide will always reduce the total initial fissile content of the fuel [16]. For this reason, higher enrichments are often required with thorium fuels (i.e., greater 
than the conventional $5 \mathrm{wt} \%{ }^{235} \mathrm{U}$ for PWR fuels but less than the nonproliferation limit of $20 \mathrm{wt} \%{ }^{235} \mathrm{U}$ $[30])$.

The objective of the modeling effort was not to optimize any particular thorium fuel concept, but rather to capture the breadth of likely thorium fuels for leading fuel cycles. Because many PWR thorium fuel concepts exist, a range of enrichments and MOX fractions were modeled. Enrichments ranged from $1 \mathrm{wt} \%{ }^{235} \mathrm{U}$ up to $20 \mathrm{wt} \%{ }^{235} \mathrm{U}$, and MOX fractions ranged from 95\%-5\% U-Th to 5\%-95\% U-Th. Enrichments were evaluated at every $1 \%$ increment between $1 \%$ and $6 \%$, and every $2 \%$ increment between $6 \%$ and $20 \%$ (i.e., $1 \mathrm{wt} \%, 2 \mathrm{wt} \%, 3 \mathrm{wt} \%, \ldots, 6 \mathrm{wt} \%, 8 \mathrm{wt} \%, 10 \mathrm{wt} \%, \ldots, 20 \mathrm{wt} \%$ ). MOX fractions were evaluated at every $5 \%$ increment between $5 \%$ and $95 \%$ (i.e., 5 wt $\%, 10$ wt $\%, 15$ $w t \%, \ldots, 95 \mathrm{wt} \%)$. Some of these combinations may produce fuel designs that would be unacceptable subject to the constraints of a particular use case, but the full range of likely homogeneous PWR fuel designs is addressed. Table 5 shows the bounding $\mathrm{UO}_{2}-\mathrm{ThO}_{2}$ homogeneous lattice PWR fuel compositions. Figure 8 shows the homogeneous PWR quarter fuel lattice.

Table 5. Bounding $\mathrm{UO}_{2}-\mathrm{ThO}_{2}$ homogeneous lattice PWR fresh fuel compositions.

\begin{tabular}{|c|c|c|c|c|c|c|c|c|c|c|c|c|}
\hline \multirow{2}{*}{ Fuel composition } & \multicolumn{7}{|l|}{ Th } & \multicolumn{5}{|c|}{$\mathrm{Pu}$} \\
\hline & ${ }^{232} \mathrm{Th}$ & ${ }^{232} U$ & ${ }^{233} \mathrm{U}$ & ${ }^{234} \mathrm{U}$ & ${ }^{235} \mathrm{U}$ & ${ }^{236} \mathrm{U}$ & ${ }^{238} \mathrm{U}$ & ${ }^{238} \mathrm{Pu}$ & ${ }^{239} \mathrm{Pu}$ & ${ }^{240} \mathrm{Pu}$ & ${ }^{241} \mathrm{Pu}$ & ${ }^{242} \mathrm{Pu}$ \\
\hline \multirow{2}{*}{$\begin{array}{l}\text { Low enriched } \\
\text { Low Th\% }\end{array}$} & $5 \%$ & \multicolumn{6}{|c|}{$95 \%$} & \multicolumn{5}{|c|}{$0 \%$} \\
\hline & $100 \%$ & $0 \%$ & $0 \%$ & $0.077 \%$ & $1 \%$ & $0.0046 \%$ & $98.9877 \%$ & $0 \%$ & $0 \%$ & $0 \%$ & $0 \%$ & $0 \%$ \\
\hline \multirow{2}{*}{$\begin{array}{l}\text { High enriched } \\
\text { Low Th\% }\end{array}$} & $5 \%$ & \multicolumn{6}{|c|}{$95 \%$} & \multicolumn{5}{|c|}{$0 \%$} \\
\hline & $100 \%$ & $0 \%$ & $0 \%$ & $0.1987 \%$ & $20 \%$ & $0.0920 \%$ & $79.7093 \%$ & $0 \%$ & $0 \%$ & $0 \%$ & $0 \%$ & $0 \%$ \\
\hline \multirow{2}{*}{$\begin{array}{l}\text { Low enriched } \\
\text { High Th\% }\end{array}$} & $95 \%$ & \multicolumn{6}{|c|}{$5 \%$} & \multicolumn{5}{|c|}{$0 \%$} \\
\hline & $100 \%$ & $0 \%$ & $0 \%$ & $0.077 \%$ & $1 \%$ & $0.0046 \%$ & $98.9877 \%$ & $0 \%$ & $0 \%$ & $0 \%$ & $0 \%$ & $0 \%$ \\
\hline \multirow{2}{*}{$\begin{array}{l}\text { High enriched } \\
\text { High Th\% }\end{array}$} & $95 \%$ & \multicolumn{6}{|c|}{$5 \%$} & \multicolumn{5}{|c|}{$0 \%$} \\
\hline & $100 \%$ & $0 \%$ & $0 \%$ & $0.1987 \%$ & $20 \%$ & $0.0920 \%$ & $79.7093 \%$ & $0 \%$ & $0 \%$ & $0 \%$ & $0 \%$ & $0 \%$ \\
\hline
\end{tabular}

Figures 9 and 10 show the resulting thorium, uranium, and plutonium mass concentrations vs. EFPDs for the homogeneous PWR U-Th MOX fuel lattices composed of 5\% Th/95\% U (Figure 9) and 95\% $\mathrm{Th} / 5 \% \mathrm{U}$ (Figure 10). The figures show that concentrations of key $\mathrm{Th}, \mathrm{U}$, and Pu nuclides can span a few orders of magnitude depending on the initial enrichment and MOX fraction.

\section{$\mathrm{UO}_{2}-\mathrm{ThO}_{2}$ Heterogeneous (Seed \& Blanket) PWR Fuel Lattice}

A leading fuel design candidate for thorium use in a PWR is a heterogeneous two-region lattice. In this design, the inner region (referred to as the seed region) contains only $\mathrm{UO}_{2}$ driver fuel, while the outer region (referred to as the blanket region) contains $\mathrm{UO}_{2}-\mathrm{ThO}_{2} \mathrm{MOX}$ fuel. The spatial separation of the driver fuel from the blanket can allow for greater breeding and burning benefits, depending on the core design and fuel cycle. The two-region concept also allows for separate reprocessing of the seed and blanket fuel pins, which is beneficial or necessary for some fuel cycle concepts. 


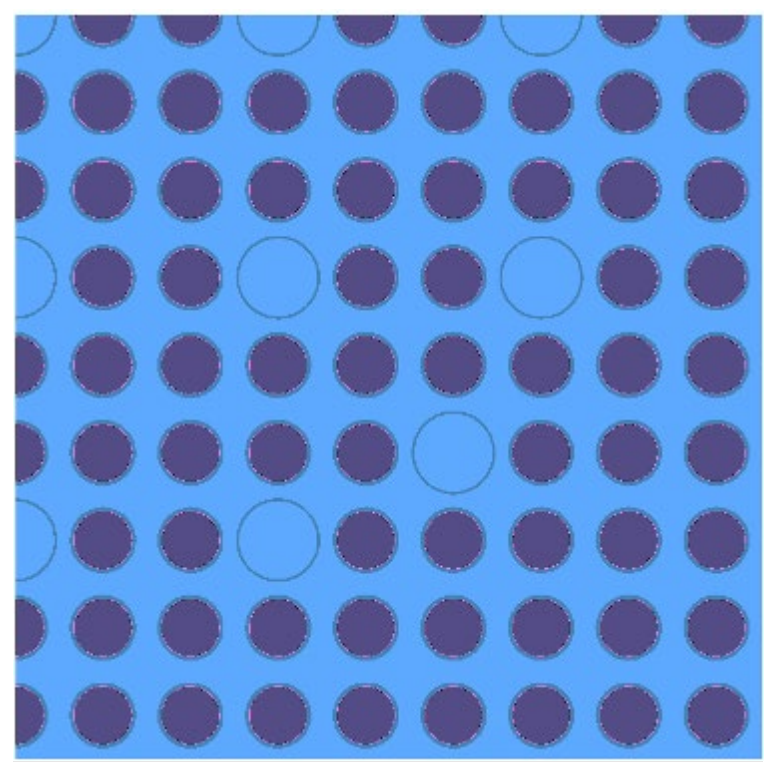

Figure 8. Homogeneous PWR quarter fuel lattice. $\mathrm{UO}_{2}-\mathrm{ThO}_{2}$-fueled pins are shown in violet. Light blue represents water (coolant/ moderator). The upper left corner is the lattice center (instrument tube location), and 8 of 24 control rod guide tubes are shown.

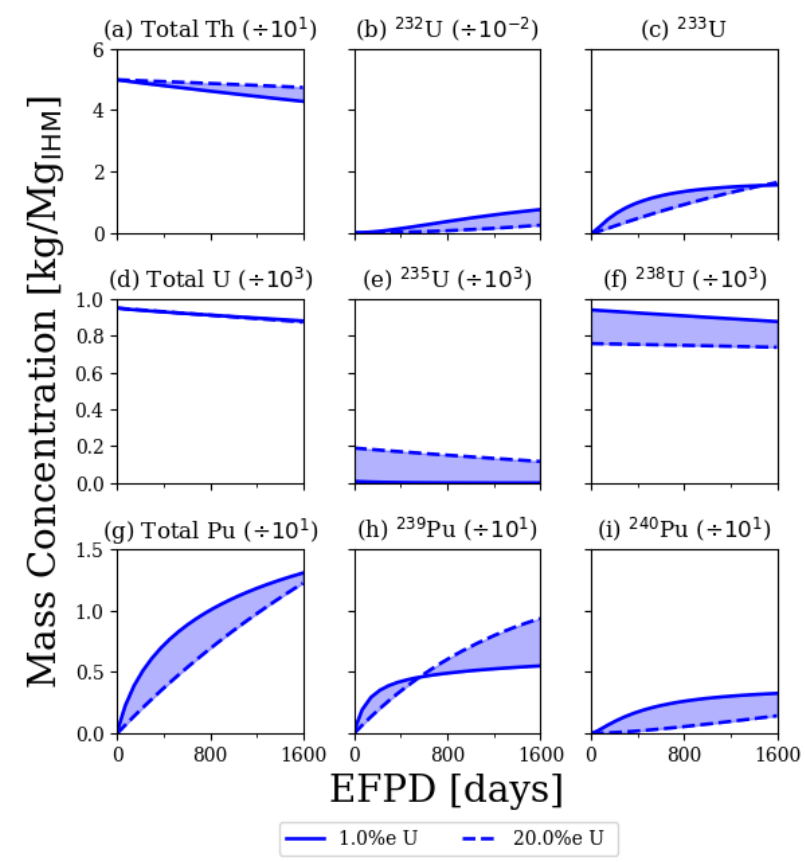

Figure 9. Th, $\mathrm{U}$, and Pu, mass concentrations vs. EFPDs for the homogeneous PWR fuel lattice composed of $5 \% \mathrm{Th} / 95 \% \mathrm{U}$ enriched from 1 to $20 \%$ enrichment. 


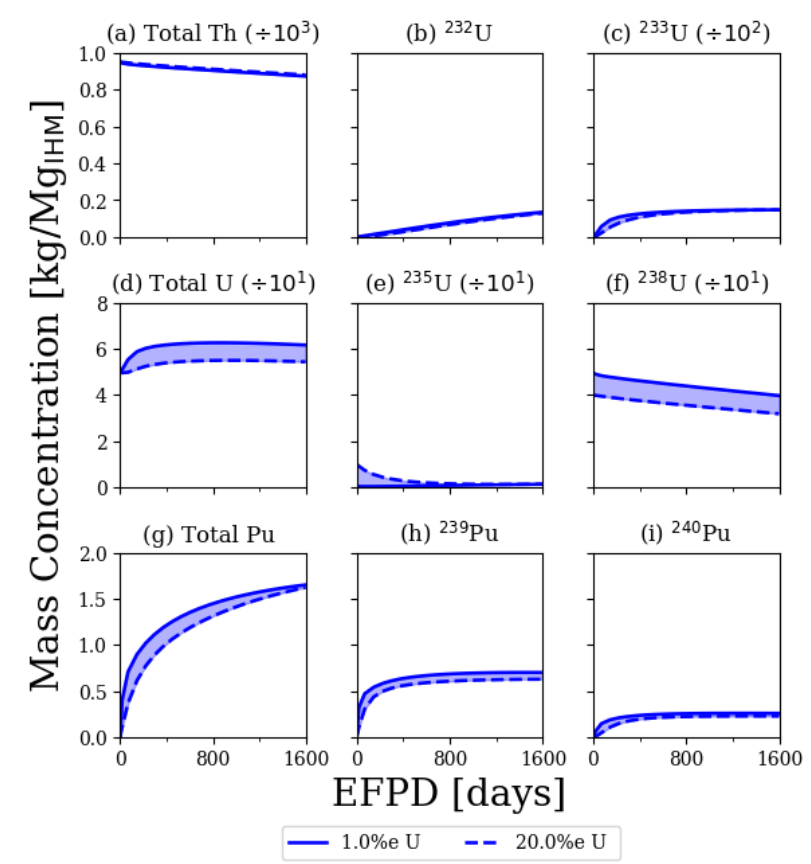

Figure 10. Th, $\mathrm{U}$, and Pu, mass concentrations vs. EFPDs for the homogeneous PWR fuel lattice composed of $95 \% \mathrm{Th} / 5 \% \mathrm{U}$ enriched from 1 to $20 \%$ enrichment.

Heterogeneous lattices provide further degrees of freedom in their design, primarily related to which pins are part of the seed and which are part of the blanket. Although several concepts exist, this study adopted a design in which all pins outside the control rodlet guide tubes are part of the blanket, while all other pins are part of the seed, as shown in Figure 11. In addition to the MOX fraction and enrichment for the U-Th MOX blanket, as described for the homogeneous lattice model, the initial uranium of the seed region can have a different enrichment from that of the blanket region. In this study, the enrichment of the seed fuel ranged from $1 \mathrm{wt} \%$ to $5 \mathrm{wt} \%{ }^{235} \mathrm{U}$, with simulations performed at $0.5 \mathrm{wt} \%$ intervals (i.e., $1.0 \mathrm{wt} \%, 1.5 \mathrm{wt} \%, 2.0 \mathrm{wt} \%, \ldots, 5.0 \mathrm{wt} \%)$. Using the same ranges and intervals as the homogeneous PWR lattice, the blanket enrichment ranged from $1 \mathrm{wt} \%$ to $5 \mathrm{wt} \%$, and the U-Th MOX fractions ranged from $15 \%$ to $90 \%$. Again, some of these combinations may produce unacceptable fuel designs for a given scenario, but it is important to note the objective of the modeling effort was not to optimize any thorium fuel concept, but rather to capture the range of likely designs. Table 6 shows the bounding $\mathrm{UO}_{2}$ heterogeneous lattice PWR seed fuel compositions, and Table 7 shows the equivalent for the blanket fuel. 


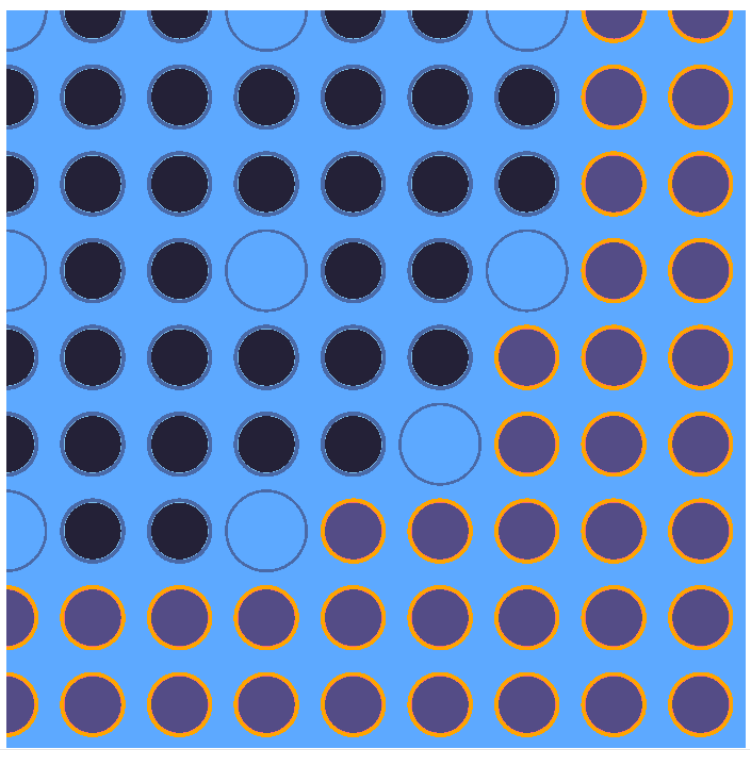

Figure 11. Heterogeneous PWR quarter fuel lattice. $\mathrm{UO}_{2}$-fueled pins (seed) are shown in black, and $\mathrm{UO}_{2}-\mathrm{ThO}_{2}-$ fueled pins (blanket) are shown in violet. Light blue represents water (coolant/ moderator). The upper left corner is the lattice center (instrument tube location), and 8 of 24 control rod guide tubes are shown.

While the blanket region could consist of pure $\mathrm{ThO}_{2}$ rather than U-Th MOX, designs of that type were not considered in this study for two primary reasons. First, the uranium bred from a pure $\mathrm{ThO}_{2}$ fuel material would be primarily ${ }^{233} \mathrm{U}$ with small but significant contaminant of ${ }^{232} \mathrm{U}$, where the ${ }^{233} \mathrm{U}$ is a material categorized as direct use. Direct-use material is defined as "nuclear material that can be used for the manufacture of nuclear explosive devices without transmutation or further enrichment" [8]. By contrast, the uranium resulting from a reprocessed U-Th MOX fuel material will be denatured (i.e., reduced in fissile content) by the other uranium isotopes present, particularly ${ }^{238} \mathrm{U}$. The denaturing of the uranium can be observed in the mass concentration plots (Figures 12-15), which show the ratio of the ${ }^{233} \mathrm{U}$ concentration to the total $\mathrm{U}$ concentration at any point in time (i.e., EFPDs). Second, adding enriched uranium to the blanket region will cause a greater fraction of power to be generated in the blanket, especially in the earlier portion of reactor power operation when power due to the fissioning of ${ }^{233} \mathrm{U}$ in the blanket is low, thus reducing lattice power peaking factors. In other words, there are good reasons using U-Th MOX in the blanket both improves the fuel design and decreases the strategic value of uranium.

Table 6. Bounding $\mathrm{UO}_{2}$ heterogeneous lattice PWR seed fuel compositions.

\begin{tabular}{|c|c|c|c|c|c|c|c|c|c|c|c|c|}
\hline \multirow{2}{*}{$\begin{array}{c}\text { Fuel } \\
\text { composition }\end{array}$} & \multicolumn{2}{|l|}{ Th } & \multicolumn{5}{|c|}{$\mathbf{U}$} & \multicolumn{5}{|c|}{$\mathbf{P u}$} \\
\hline & ${ }^{232} \mathrm{Th}$ & ${ }^{232} \mathrm{U}$ & ${ }^{233} \mathrm{U}$ & ${ }^{234} \mathrm{U}$ & ${ }^{235} \mathrm{U}$ & ${ }^{236} \mathrm{U}$ & ${ }^{238} \mathrm{U}$ & ${ }^{238} \mathrm{Pu}$ & ${ }^{239} \mathrm{Pu}$ & ${ }^{240} \mathrm{Pu}$ & ${ }^{241} \mathrm{Pu}$ & ${ }^{242} \mathrm{Pu}$ \\
\hline \multirow[t]{2}{*}{ Low enriched } & $0.0 \%$ & \multicolumn{6}{|c|}{$100.0 \%$} & \multicolumn{5}{|c|}{$0 \%$} \\
\hline & $0.0 \%$ & $0.0 \%$ & $0.0 \%$ & $0.077 \%$ & $1.0 \%$ & $0.0046 \%$ & 98.9877\% & $0 \%$ & $0 \%$ & $0 \%$ & $0 \%$ & $0 \%$ \\
\hline \multirow[t]{2}{*}{ High enriched } & $0.0 \%$ & \multicolumn{6}{|c|}{$100.0 \%$} & \multicolumn{5}{|c|}{$0 \%$} \\
\hline & $0.0 \%$ & $0.0 \%$ & $0.0 \%$ & $0.0442 \%$ & $5 \%$ & $0.023 \%$ & $94.9328 \%$ & $0 \%$ & $0 \%$ & $0 \%$ & $0 \%$ & $0 \%$ \\
\hline
\end{tabular}


Table 7. Bounding $\mathrm{UO}_{2}-\mathrm{ThO}_{2}$ heterogeneous lattice PWR blanket fuel compositions.

\begin{tabular}{|c|c|c|c|c|c|c|c|c|c|c|c|c|}
\hline \multirow{2}{*}{ Fuel composition } & \multicolumn{2}{|l|}{ Th } & \multicolumn{5}{|c|}{$\mathbf{U}$} & \multicolumn{5}{|c|}{$\mathbf{P u}$} \\
\hline & ${ }^{232} \mathrm{Th}$ & ${ }^{232} \mathrm{U}$ & ${ }^{233} \mathrm{U}$ & ${ }^{234} U$ & ${ }^{235} \mathrm{U}$ & ${ }^{236} \mathrm{U}$ & ${ }^{238} U$ & ${ }^{238} \mathrm{Pu}$ & ${ }^{239} \mathrm{Pu}$ & ${ }^{240} \mathrm{Pu}$ & ${ }^{241} \mathrm{Pu}$ & ${ }^{242} \mathrm{Pu}$ \\
\hline \multirow{2}{*}{$\begin{array}{l}\text { Low enriched } \\
\text { Low Th\% }\end{array}$} & $10 \%$ & \multicolumn{6}{|c|}{$90 \%$} & \multicolumn{5}{|c|}{$0 \%$} \\
\hline & $100 \%$ & $0 \%$ & $0 \%$ & $0.077 \%$ & $1 \%$ & $0.0046 \%$ & $98.9877 \%$ & $0 \%$ & $0 \%$ & $0 \%$ & $0 \%$ & $0 \%$ \\
\hline \multirow{2}{*}{$\begin{array}{l}\text { High enriched } \\
\text { Low Th\% }\end{array}$} & $10 \%$ & \multicolumn{6}{|c|}{$90 \%$} & \multicolumn{5}{|c|}{$0 \%$} \\
\hline & $100 \%$ & $0 \%$ & $0 \%$ & $0.1987 \%$ & $20 \%$ & $0.0920 \%$ & $79.7093 \%$ & $0 \%$ & $0 \%$ & $0 \%$ & $0 \%$ & $0 \%$ \\
\hline \multirow{2}{*}{$\begin{array}{l}\text { Low enriched } \\
\text { High Th\% }\end{array}$} & $85 \%$ & \multicolumn{6}{|c|}{$15 \%$} & \multicolumn{5}{|c|}{$0 \%$} \\
\hline & $100 \%$ & $0 \%$ & $0 \%$ & $0.077 \%$ & $1 \%$ & $0.0046 \%$ & $98.9877 \%$ & $0 \%$ & $0 \%$ & $0 \%$ & $0 \%$ & $0 \%$ \\
\hline \multirow{2}{*}{$\begin{array}{l}\text { High enriched } \\
\text { High Th\% }\end{array}$} & $85 \%$ & \multicolumn{6}{|c|}{$15 \%$} & \multicolumn{5}{|c|}{$0 \%$} \\
\hline & $100 \%$ & $0 \%$ & $0 \%$ & $0.1987 \%$ & $20 \%$ & $0.0920 \%$ & $79.7093 \%$ & $0 \%$ & $0 \%$ & $0 \%$ & $0 \%$ & $0 \%$ \\
\hline
\end{tabular}

Figures $12-15$ show the resulting mass concentrations vs. EFPDs for the heterogeneous PWR fuel lattice cases simulated. Figure 12 shows results for the heterogeneous PWR fuel lattice blanket composed of $85 \% \mathrm{Th} / 15 \% \mathrm{U}$, with the $15 \% \mathrm{U}$ ranging from $1 \mathrm{wt} \%$ to $5 \mathrm{wt} \%{ }^{235} \mathrm{U}$ enrichment and an enriched $\mathrm{UO}_{2}$ seed region with enrichment of $5 \mathrm{wt} \%{ }^{235} \mathrm{U}$. Figure 13 shows results for the heterogeneous blanket composed of $10 \% \mathrm{Th} / 90 \% \mathrm{U}$ with the same seed enrichment.

Figures 14 and 15 show results for the heterogeneous PWR fuel lattice blanket with an enriched $\mathrm{UO}_{2}$ seed region of $1 \mathrm{wt} \%{ }^{235} \mathrm{U}$, using blanket compositions of $85 \% \mathrm{U}$ and $15 \%$ Th, respectively, with the remainder $\mathrm{UO}_{2}$ enriched from $1 \mathrm{wt} \%$ to $5 \mathrm{wt} \%{ }^{235} \mathrm{U}$. As observed in the figures, the seed composition does not appear to have a strong effect on the quantities of ${ }^{232} \mathrm{U}$ and ${ }^{233} \mathrm{U}$ produced at the modeled range of enrichment.

\subsubsection{Recycle PWR Thorium Fuels}

The recycle PWR fuel designs have ${ }^{233} \mathrm{U}$ in the initial fuel compositions, which implies at least one previous generation of thorium fuel where the ${ }^{233} \mathrm{U}$ was bred. Upon discharge, the uranium vector from a U-Th MOX fuel contains all six long-lived uranium isotopes in some combination that depends not only on its initial fresh fuel composition but also on its discharge burnup. After or during the reprocessing stage, the discharged uranium can be blended with additional uranium streams, further altering the uranium vector. As was shown for the initial-cycle thorium fuels, the number of enrichment and MOX fraction parameters escalates for even simplified lattice designs, so parameterizing recycle lattice designs by those parameters as well as the discharge burnup and possible blend streams quickly becomes untenable for even a limited number of cases. Instead of a parameter study, the recycle PWR uranium vectors used herein were adopted from previous studies [31]. 


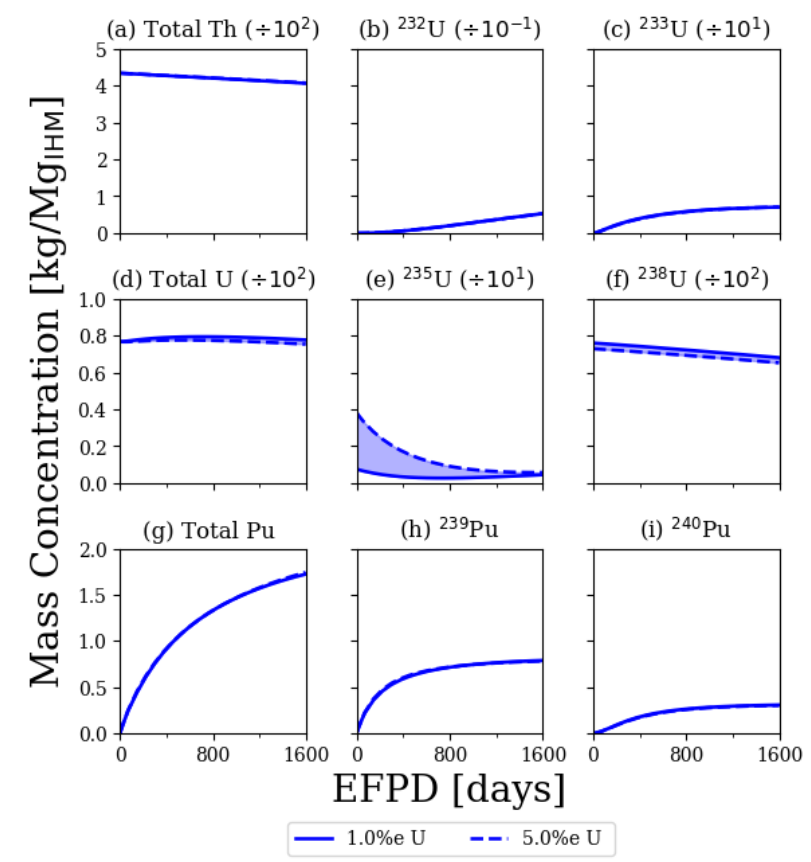

Figure 12. Th, $\mathrm{U}$, and $\mathrm{Pu}$, mass concentrations vs. EFPDs for the heterogeneous PWR fuel lattice blanket composed of $85 \% \mathrm{Th} / 15 \% \mathrm{U}$ at a range of enriched from $1 \%$ to $5 \%$ enrichment, with a $5 \%$ enriched $\mathrm{UO}_{2}$ seed region.

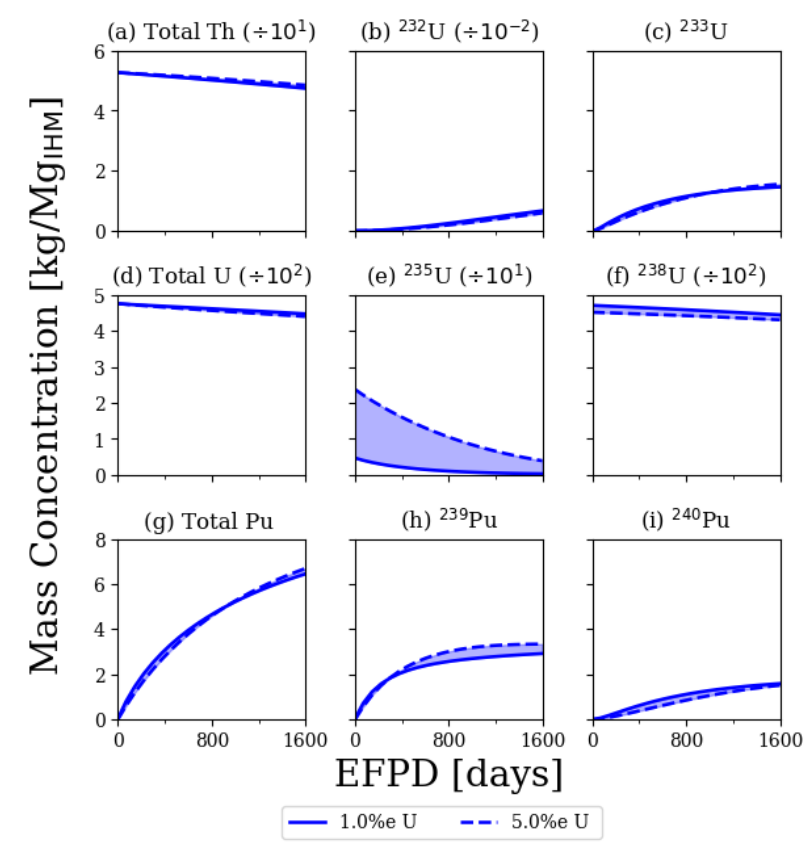

Figure 13. Th, $\mathrm{U}$, and $\mathrm{Pu}$, mass concentrations vs. EFPDs for the heterogeneous PWR fuel lattice blanket composed of $10 \% \mathrm{Th} / 90 \% \mathrm{U}$ at a range of enriched from $1 \%$ to $5 \%$ enrichment, with a $5 \%$ enriched $\mathrm{UO}_{2}$ seed region. 


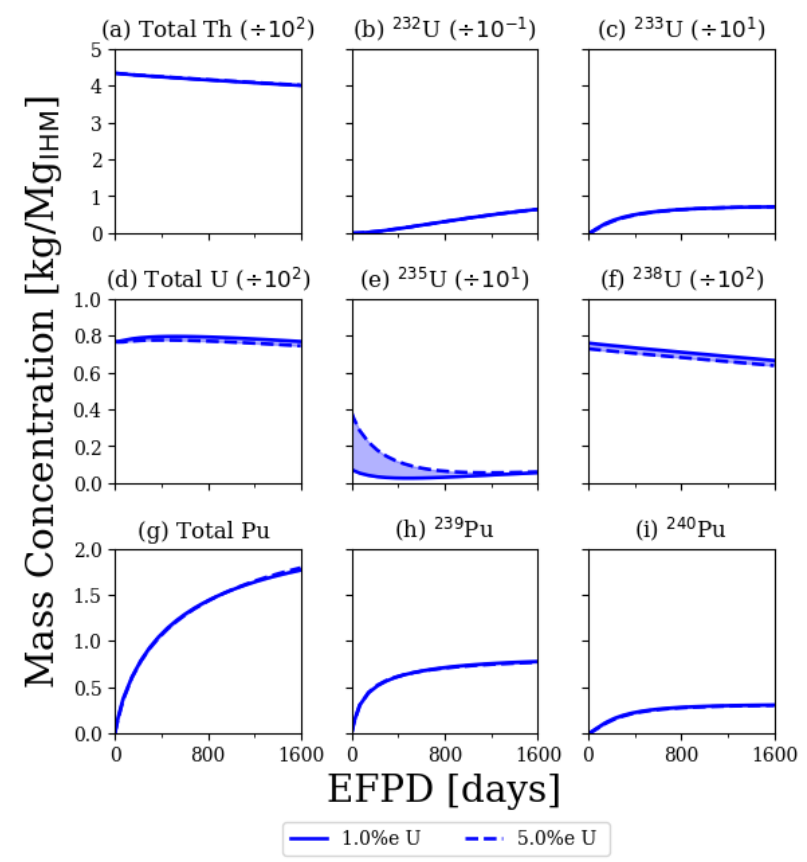

Figure 14. Th, $\mathrm{U}$, and $\mathrm{Pu}$, mass concentrations vs. EFPDs for the heterogeneous PWR fuel lattice blanket composed of $85 \% \mathrm{Th} / 15 \% \mathrm{U}$ at a range of enriched from $1 \%$ to $5 \%$ enrichment, with a $1 \%$ enriched $\mathrm{UO}_{2}$ seed region.

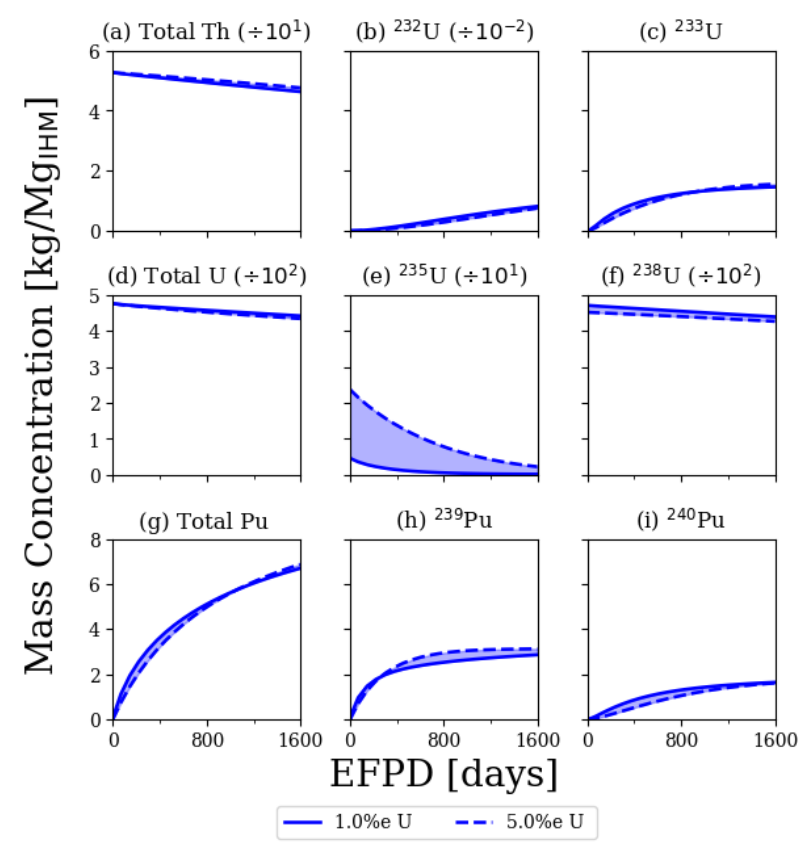

Figure 15. Th, $\mathrm{U}$, and $\mathrm{Pu}$, mass concentrations vs. EFPDs for the heterogeneous PWR fuel lattice blanket composed of $10 \% \mathrm{Th} / 90 \% \mathrm{U}$ enriched from $1 \%$ to $5 \%$, with a $1 \%$ enriched $\mathrm{UO}_{2}$ seed region.

For fuel designs where the breeding ratio is less than 1.0, more fissile isotopes will be consumed than bred, so the additional uranium stream can be used to compensate for, or make up, the fissile content of the reprocessed uranium. This makeup material must have a greater fissile content than the reprocessed uranium if it is to be used as driver fuel in subsequent generations. Historical examples 
include the Elk River reactor, which employed U-Th MOX with $92 \%{ }^{235} \mathrm{U}$; the Indian Point I reactor, which employed U-Th MOX with $93 \%{ }^{235} \mathrm{U}$; and the Shippingport reactor, which employed U-Th MOX $98 \%{ }^{233} \mathrm{U}$ [32]. Alternatively, the discharged uranium could be mixed with plutonium to increase its fissile content. Even reactor-grade plutonium has a high fissile content compared to LEU, so U-Pu MOX would have certain fuel cycle benefits in terms of material utilization and disposition. U-Pu, Th-Pu, and U-Th-Pu MOX fuel materials were not simulated for these recycle PWR fuel designs, but these combinations were simulated for other reactor types.

\section{$\mathrm{UO}_{2}-\mathrm{ThO}_{2}$ PWR Fuel with ${ }^{233} \mathrm{U}$ Makeup}

In previous studies, Arthur [31] considered thorium use in PWRs where the initial U-Th MOX fuel (Generation 0 ) consisted of $81.3 \mathrm{wt} \%$ natural thorium (100\% ${ }^{232} \mathrm{Th}$ ) and $18.7 \mathrm{wt} \%$ enriched uranium (i.e., $79.98 w t \%{ }^{238} \mathrm{U}, 19.86 \mathrm{wt} \%{ }^{235} \mathrm{U}$, and $0.16 \mathrm{wt} \%{ }^{234} \mathrm{U}$ ). The fuel depletion was simulated over five subsequent recycle generations. After depletion, makeup uranium was blended with the discharged uranium to increase its fissile content. Two sources of makeup uranium were considered: ${ }^{233} \mathrm{U}$ (with minor uranium contaminants) produced from a hypothetical thorium breeder blanket, and $93 \mathrm{wt} \%$ ${ }^{235} \mathrm{U}$, presumably part of a weapons material disposition program.

Table 8 lists the initial U-Th MOX fuel compositions for a homogenous PWR fuel lattice design for recycle Generations 1 and 5. Although the fuel composition is different, the geometry of these homogeneous models is identical to that shown in Figure 6 . Only fuels for the initial and final recycle generations were selected for modeling because these fuel compositions bracket the compositions from the intermediate generations. Over the five generations, the fuel composition evolves, but the Th- $U$ ratio, ${ }^{233} \mathrm{U}$ content, and ${ }^{238} \mathrm{U}$ content change relatively little. The largest differences from Generation 1 to Generation 5 are in the ${ }^{234} \mathrm{U},{ }^{235} \mathrm{U}$, and ${ }^{236} \mathrm{U}$ content as these higher $\mathrm{U}$ isotopes build in, along with a more gradual increase in ${ }^{232} \mathrm{U}$. This can be observed in Tables $8-10$ and Figures $16-18$. The tables show the small changes in the fresh fuel composition from Generation 1 to Generation 5, while the figures show the changes between the generations (i.e., the shaded region between traces) during depletion.

For the heterogeneous models, Generations 1 and $5{ }^{233} \mathrm{U}$-Th MOX compositions can be used in either the seed region or the blanket region. Although the fuel composition is different, the geometry of these heterogeneous models is identical to that shown in Figure 8. Table 9 lists the fuel compositions for the ${ }^{233} \mathrm{U}$-Th MOX material employed in the blanket region, with the seed region using a typical PWR fuel of $4.0 \mathrm{wt} \%{ }^{235} \mathrm{U}$ enrichment. Table 10 lists the fuel compositions for the ${ }^{233} \mathrm{U}$-Th MOX material employed in the seed region, with the blanket region using ${ }^{235} \mathrm{U}$-Th MOX consisting of $70 \mathrm{wt} \%$ Th and $30 \% \mathrm{U}$ enriched to $4.0 \mathrm{wt} \%{ }^{235} \mathrm{U}$.

Table 8. $\mathrm{UO}_{2}-\mathrm{ThO}_{2}$ homogeneous PWR fuel lattice with ${ }^{233} \mathrm{U}$ makeup.

\begin{tabular}{|c|c|c|c|c|c|c|c|c|c|c|c|c|}
\hline \multirow{2}{*}{$\begin{array}{c}\text { Fuel } \\
\text { composition }\end{array}$} & Th & \multicolumn{6}{|c|}{$\mathbf{U}$} & \multicolumn{5}{|c|}{$\mathbf{P u}$} \\
\hline & ${ }^{232} \mathrm{Th}$ & ${ }^{232} \mathrm{U}$ & ${ }^{233} \mathrm{U}$ & ${ }^{234} \mathrm{U}$ & ${ }^{235} \mathrm{U}$ & ${ }^{236} \mathrm{U}$ & ${ }^{238} \mathrm{U}$ & ${ }^{238} \mathrm{Pu}$ & ${ }^{239} \mathrm{Pu}$ & ${ }^{240} \mathrm{Pu}$ & ${ }^{241} \mathrm{Pu}$ & ${ }^{242} \mathrm{Pu}$ \\
\hline \multirow{2}{*}{$\begin{array}{l}\text { Generation } \\
1\end{array}$} & $73.2 \%$ & \multicolumn{6}{|c|}{$26.8 \%$} & \multicolumn{5}{|c|}{$0 \%$} \\
\hline & $100 \%$ & $0.048 \%$ & $11.634 \%$ & $0.838 \%$ & $0.229 \%$ & $0.004 \%$ & $87.246 \%$ & $0 \%$ & $0 \%$ & $0 \%$ & $0 \%$ & $0 \%$ \\
\hline \multirow{2}{*}{$\begin{array}{l}\text { Generation } \\
5\end{array}$} & $72 \%$ & \multicolumn{6}{|c|}{$28 \%$} & \multicolumn{5}{|c|}{$0 \%$} \\
\hline & $100 \%$ & $0.067 \%$ & $11.827 \%$ & $3.626 \%$ & $1.300 \%$ & $0.555 \%$ & $87.333 \%$ & $0 \%$ & $0 \%$ & $0 \%$ & $0 \%$ & $0 \%$ \\
\hline
\end{tabular}


Table 9. $\mathrm{UO}_{2}-\mathrm{ThO}_{2}$ heterogeneous (seed and blanket) PWR fuel lattice with ${ }^{233} \mathrm{U}$ makeup and constant seed composition.

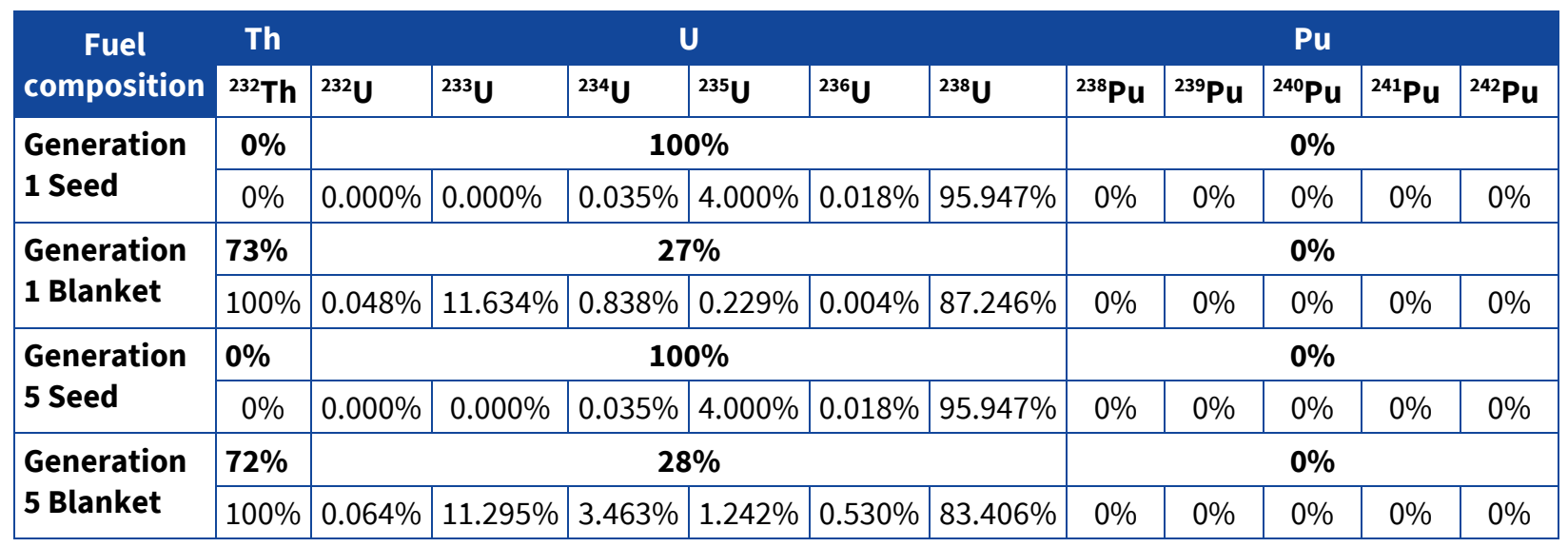

Table 10. $\mathrm{UO}_{2}-\mathrm{ThO}_{2}$ heterogeneous (seed and blanket) PWR fuel lattice with ${ }^{233} \mathrm{U}$ makeup and constant blanket composition.

\begin{tabular}{|c|c|c|c|c|c|c|c|c|c|c|c|c|}
\hline \multirow{2}{*}{$\begin{array}{c}\text { Fuel } \\
\text { composition }\end{array}$} & \multirow{2}{*}{$\begin{array}{c}\text { Th } \\
{ }^{232} \mathrm{Th}\end{array}$} & \multicolumn{6}{|c|}{$\mathbf{U}$} & \multicolumn{5}{|c|}{$\mathrm{Pu}$} \\
\hline & & ${ }^{232} \mathrm{U}$ & ${ }^{233} \mathrm{U}$ & ${ }^{234} \mathrm{U}$ & ${ }^{235} \mathrm{U}$ & ${ }^{236} \mathrm{U}$ & ${ }^{238} \mathrm{U}$ & ${ }^{238} \mathrm{Pu}$ & ${ }^{239} \mathrm{Pu}$ & ${ }^{240} \mathrm{Pu}$ & ${ }^{241} \mathrm{Pu}$ & ${ }^{242} \mathrm{Pu}$ \\
\hline \multirow{2}{*}{$\begin{array}{l}\text { Generation } 1 \\
\text { Seed }\end{array}$} & $0 \%$ & \multicolumn{6}{|c|}{$100 \%$} & \multicolumn{5}{|c|}{$0 \%$} \\
\hline & $0 \%$ & $0.048 \%$ & $11.634 \%$ & $0.838 \%$ & $0.229 \%$ & $0.004 \%$ & $87.246 \%$ & $0 \%$ & $0 \%$ & $0 \%$ & $0 \%$ & $0 \%$ \\
\hline \multirow{2}{*}{$\begin{array}{l}\text { Generation } 1 \\
\text { Blanket }\end{array}$} & $70 \%$ & \multicolumn{6}{|c|}{$30 \%$} & \multicolumn{5}{|c|}{$0 \%$} \\
\hline & $100 \%$ & $0.000 \%$ & $0.000 \%$ & $0.035 \%$ & $4.000 \%$ & $0.018 \%$ & $95.947 \%$ & $0 \%$ & $0 \%$ & $0 \%$ & $0 \%$ & $0 \%$ \\
\hline \multirow{2}{*}{$\begin{array}{l}\text { Generation } 5 \\
\text { Seed }\end{array}$} & $0 \%$ & \multicolumn{6}{|c|}{$100 \%$} & \multicolumn{5}{|c|}{$0 \%$} \\
\hline & $\begin{array}{c}0.064 \\
\%\end{array}$ & $11.295 \%$ & $3.463 \%$ & $1.242 \%$ & $0.530 \%$ & $83.406 \%$ & $0.064 \%$ & $0 \%$ & $0 \%$ & $0 \%$ & $0 \%$ & $0 \%$ \\
\hline \multirow{2}{*}{$\begin{array}{l}\text { Generation } 5 \\
\text { Blanket }\end{array}$} & $70 \%$ & \multicolumn{6}{|c|}{$30 \%$} & \multicolumn{5}{|c|}{$0 \%$} \\
\hline & $100 \%$ & $0.000 \%$ & $0.000 \%$ & $0.035 \%$ & $4.000 \%$ & $0.018 \%$ & $95.947 \%$ & $0 \%$ & $0 \%$ & $0 \%$ & $0 \%$ & $0 \%$ \\
\hline
\end{tabular}

Figures 16-18 show the resulting mass concentrations vs. EFPDs for the PWR fuel lattice cases simulated. Figure 16 shows results for the homogeneous PWR fuel lattice with ${ }^{233} \mathrm{U}$ makeup for recycle Generations 1-5. Figure 17 shows results for the heterogeneous PWR fuel lattice with ${ }^{233} \mathrm{U}$ makeup and constant seed composition ( $4 \mathrm{wt} \%$ enriched $\mathrm{UO}_{2}$ ) for Generations $1-5$. Figure 18 shows results for the heterogenous PWR fuel lattice with ${ }^{23} \mathrm{U}$ makeup and constant blanket composition ( $\left.70 \% \mathrm{Th} / 30 \% \mathrm{U}\right)$ for Generations 1-5. 


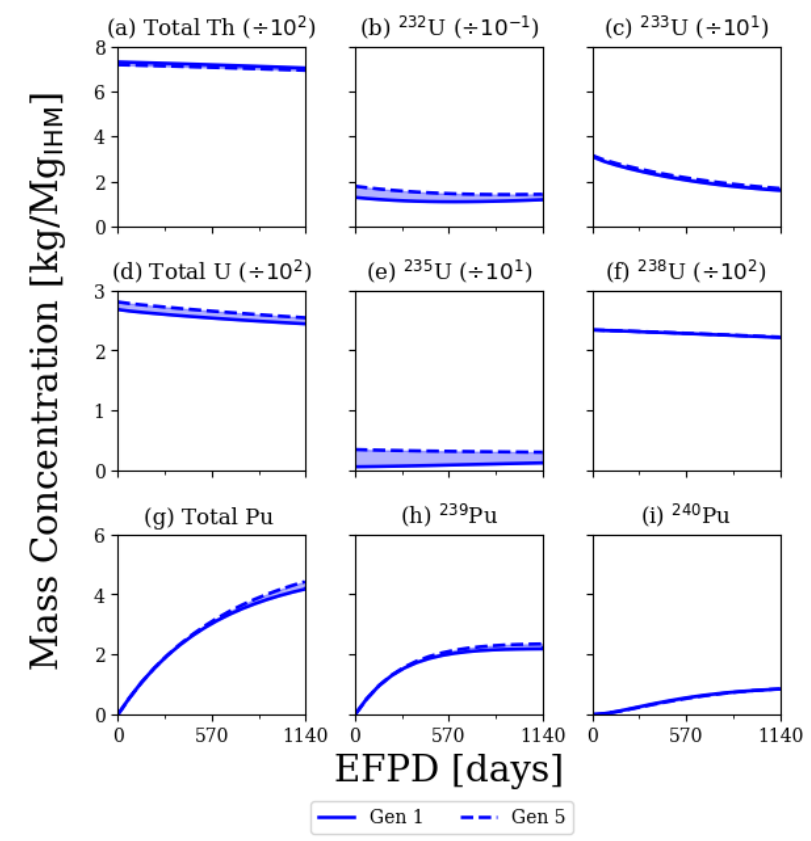

Figure 16. Th, $\mathrm{U}$, and $\mathrm{Pu}$, mass concentrations vs. EFPDs for the homogeneous PWR fuel lattice with ${ }^{233} \mathrm{U}$ makeup for recycle Generations 1-5.

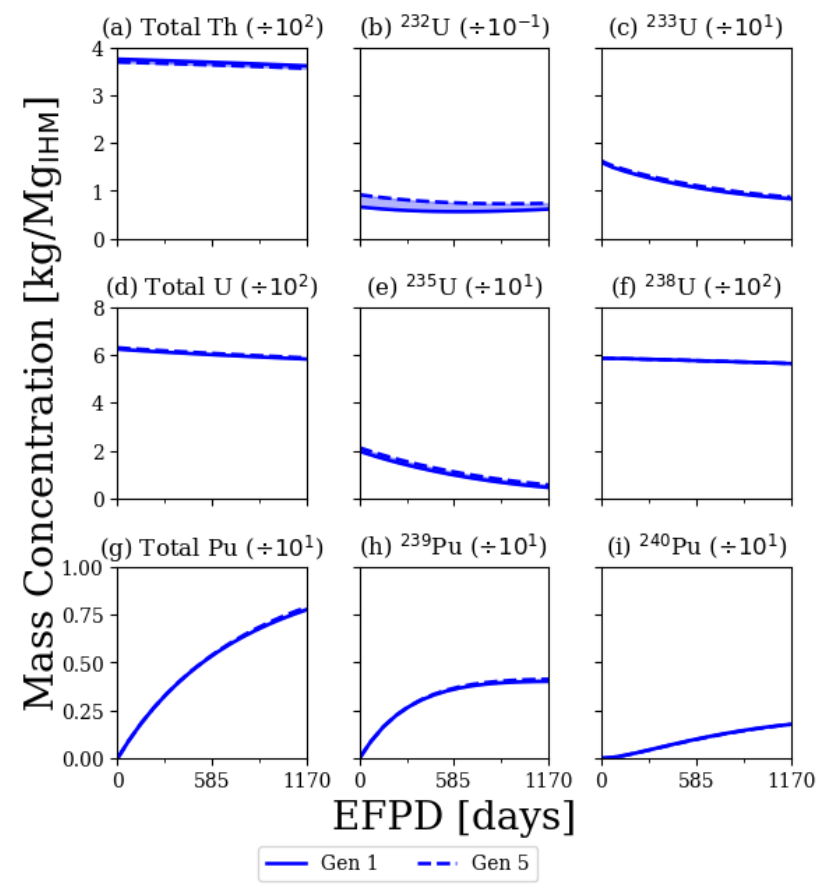

Figure 17. Th, $U$, and $P u$, mass concentrations vs. EFPDs for the heterogeneous PWR fuel lattice with ${ }^{233} U$ makeup and constant seed composition ( $4 \mathrm{wt} \%$ enriched $\mathrm{UO}_{2}$ ) for recycle Generations 1-5. 


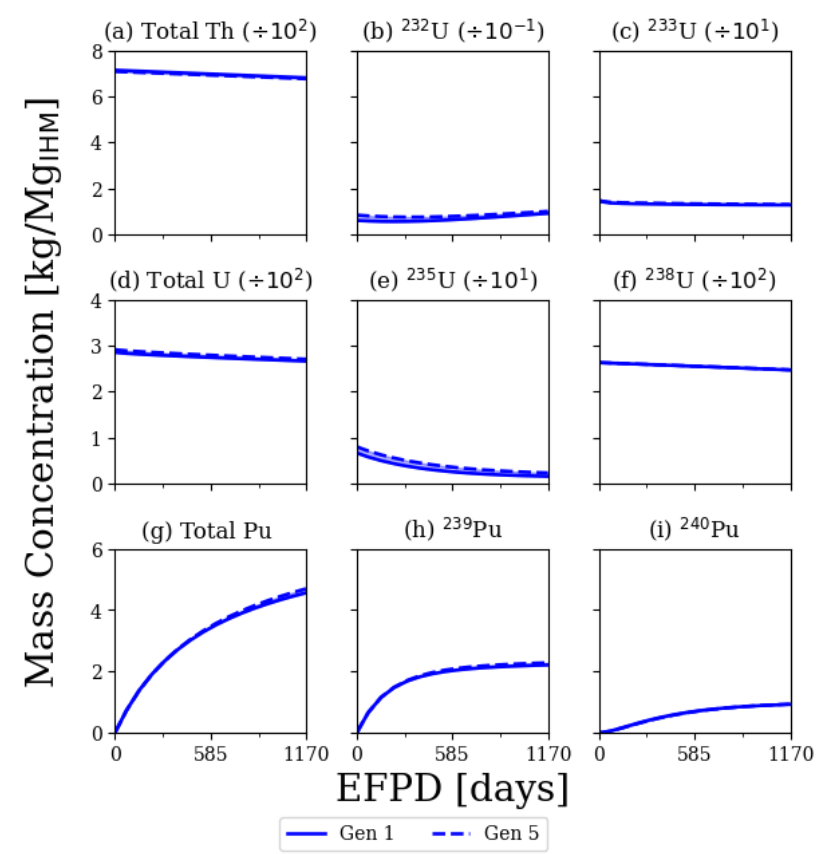

Figure 18. Th, $\mathrm{U}$, and $\mathrm{Pu}$, mass concentrations vs. EFPDs for the heterogeneous PWR fuel lattice with ${ }^{233} \mathrm{U}$ makeup and constant blanket composition $(70 \% \mathrm{Th} / 30 \% \mathrm{U})$ for recycle Generations 1-5.

\section{$\mathrm{UO}_{2}-\mathrm{ThO}_{2}$ PWR Fuel with ${ }^{235} \mathrm{U}$ Makeup}

Recycle $\mathrm{UO}_{2}-\mathrm{ThO}_{2}$ PWR fuels were also simulated using $93 \mathrm{wt} \%{ }^{235} \mathrm{U}$ as the makeup material with model variations analogous to those made using ${ }^{233} \mathrm{U}$. Over Generations $1-5$, the Th- $U$ ratio evolved only slightly, along with small relative differences in the ${ }^{233} \mathrm{U},{ }^{235} \mathrm{U}$, and ${ }^{238} \mathrm{U}$ content. The largest relative differences between Generations 1 and 5 are in the ${ }^{232} U,{ }^{234} U$, and ${ }^{236} U$ content.

Table 11 lists the initial U-Th MOX fuel compositions for a homogenous PWR fuel lattice design for Generations 1 and 5. Although the fuel composition is different, the geometry of these homogeneous models is identical to that shown in Figure 2. Table 12 lists the fuel compositions for the ${ }^{233} \mathrm{U}$-Th MOX material employed in the blanket region, with the seed region using a typical PWR fuel of $4.0 \mathrm{wt} \%$ enrichment. Table 13 lists the fuel compositions for the ${ }^{233} \mathrm{U}$-Th MOX material employed in the seed region, with the blanket region using ${ }^{235} \mathrm{U}$-Th MOX consisting of $70 \mathrm{wt} \%$ Th and $30 \% \mathrm{U}$ enriched to $4.0 \mathrm{wt} \%{ }^{235} \mathrm{U}$. Although the fuel composition is different, the geometry of these heterogeneous models is identical to that shown in Figure 3.

Table 11. $\mathrm{UO}_{2}-\mathrm{ThO}_{2}$ homogeneous PWR fuel lattice with ${ }^{235} \mathrm{U}$ makeup.

\begin{tabular}{|c|c|c|c|c|c|c|c|c|c|c|c|c|}
\hline \multirow{2}{*}{$\begin{array}{c}\text { Fuel } \\
\text { composition }\end{array}$} & \multicolumn{4}{|l|}{ Th } & \multicolumn{3}{|l|}{$\mathbf{U}$} & \multicolumn{5}{|c|}{ Pu } \\
\hline & ${ }^{232} \mathrm{Th}$ & ${ }^{232} \mathrm{U}$ & ${ }^{233} \mathrm{U}$ & ${ }^{234} \mathrm{U}$ & ${ }^{235} \mathrm{U}$ & ${ }^{236} \mathrm{U}$ & ${ }^{238} \mathrm{U}$ & ${ }^{238} \mathrm{Pu}$ & ${ }^{239} \mathrm{Pu}$ & ${ }^{240} \mathrm{Pu}$ & ${ }^{241} \mathrm{Pu}$ & ${ }^{242} \mathrm{Pu}$ \\
\hline \multirow{2}{*}{$\begin{array}{l}\text { Generation } \\
1\end{array}$} & $76.3 \%$ & \multicolumn{6}{|c|}{$23.7 \%$} & \multicolumn{5}{|c|}{$0 \%$} \\
\hline & $100 \%$ & $0.012 \%$ & $4.615 \%$ & $0.538 \%$ & $13.072 \%$ & $2.288 \%$ & $79.476 \%$ & $0 \%$ & $0 \%$ & $0 \%$ & $0 \%$ & $0 \%$ \\
\hline \multirow{2}{*}{$\begin{array}{l}\text { Generation } \\
5\end{array}$} & $73.5 \%$ & \multicolumn{6}{|c|}{$26.5 \%$} & \multicolumn{5}{|c|}{$0 \%$} \\
\hline & $100 \%$ & $0.024 \%$ & $5.810 \%$ & $2.089 \%$ & $11.241 \%$ & $5.622 \%$ & $75.214 \%$ & $0 \%$ & $0 \%$ & $0 \%$ & $0 \%$ & $0 \%$ \\
\hline
\end{tabular}


Table 12. $\mathrm{UO}_{2}-\mathrm{ThO}_{2}$ heterogeneous (seed and blanket) PWR fuel lattice with ${ }^{235} \mathrm{U}$ makeup and constant seed composition.

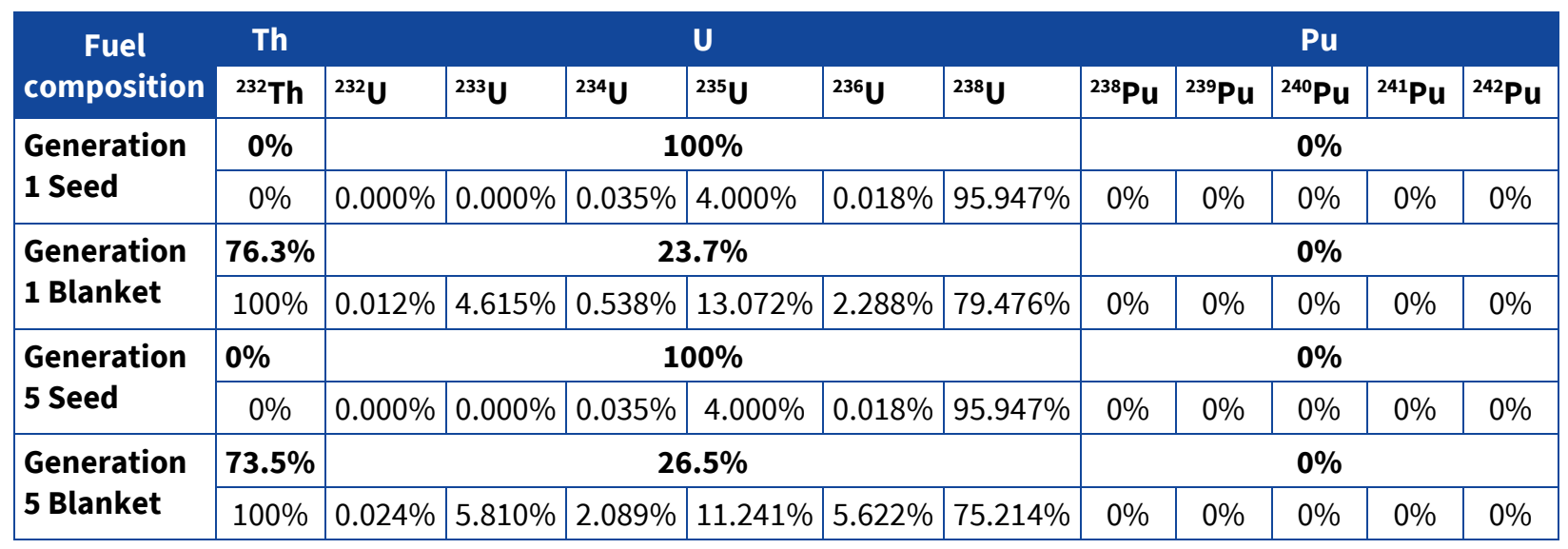

Table 13. $\mathrm{UO}_{2}-\mathrm{ThO}_{2}$ heterogeneous (seed and blanket) PWR fuel lattice with ${ }^{235} \mathrm{U}$ makeup and constant blanket composition.

\begin{tabular}{|c|c|c|c|c|c|c|c|c|c|c|c|c|}
\hline \multirow{2}{*}{$\begin{array}{c}\text { Fuel } \\
\text { composition }\end{array}$} & \multicolumn{4}{|l|}{ Th } & \multicolumn{3}{|l|}{$\mathbf{U}$} & \multicolumn{5}{|c|}{ Pu } \\
\hline & ${ }^{232} \mathrm{Th}$ & ${ }^{232} \mathrm{U}$ & ${ }^{233} \mathrm{U}$ & ${ }^{234} \mathrm{U}$ & ${ }^{235} \mathrm{U}$ & ${ }^{236} \mathrm{U}$ & ${ }^{238} \mathrm{U}$ & ${ }^{238} \mathrm{Pu}$ & ${ }^{239} \mathrm{Pu}$ & ${ }^{240} \mathrm{Pu}$ & ${ }^{241} \mathrm{Pu}$ & ${ }^{242} \mathrm{Pu}$ \\
\hline \multirow{2}{*}{$\begin{array}{l}\text { Generation } \\
1 \text { Seed }\end{array}$} & $76.3 \%$ & \multicolumn{6}{|c|}{$23.7 \%$} & \multicolumn{5}{|c|}{$0 \%$} \\
\hline & $100 \%$ & $0.012 \%$ & $4.615 \%$ & $0.538 \%$ & $13.072 \%$ & $2.288 \%$ & $79.476 \%$ & $0 \%$ & $0 \%$ & $0 \%$ & $0 \%$ & $0 \%$ \\
\hline \multirow{2}{*}{$\begin{array}{l}\text { Generation } \\
1 \text { Blanket }\end{array}$} & $70 \%$ & \multicolumn{6}{|c|}{$30 \%$} & \multicolumn{5}{|c|}{$0 \%$} \\
\hline & $100 \%$ & $0.000 \%$ & $0.000 \%$ & $0.035 \%$ & $4.000 \%$ & $0.018 \%$ & $95.947 \%$ & $0 \%$ & $0 \%$ & $0 \%$ & $0 \%$ & $0 \%$ \\
\hline \multirow{2}{*}{$\begin{array}{l}\text { Generation } \\
5 \text { Seed }\end{array}$} & $0 \%$ & \multicolumn{6}{|c|}{$100 \%$} & \multicolumn{5}{|c|}{$0 \%$} \\
\hline & $0 \%$ & $0.024 \%$ & $5.810 \%$ & $2.089 \%$ & $11.241 \%$ & $5.622 \%$ & $75.214 \%$ & $0 \%$ & $0 \%$ & $0 \%$ & $0 \%$ & $0 \%$ \\
\hline \multirow{2}{*}{$\begin{array}{l}\text { Generation } \\
5 \text { Blanket }\end{array}$} & $70 \%$ & \multicolumn{6}{|c|}{$30 \%$} & \multicolumn{5}{|c|}{$0 \%$} \\
\hline & $100 \%$ & $0.000 \%$ & $0.000 \%$ & $0.035 \%$ & $4.000 \%$ & $0.018 \%$ & 95.947\% & $0 \%$ & $0 \%$ & $0 \%$ & $0 \%$ & $0 \%$ \\
\hline
\end{tabular}

Figures 19-21 show the resulting mass concentrations for the simulated fuel cases. Again, there is little difference for most of the mass concentrations between Generations 1 and 5 over the course of the fuel cycle.

\subsubsection{Pressurized Heavy Water Reactors}

As of 2020 , PHWRs comprise $~ 10.7 \%$ of the world's power reactors and provide $6.1 \%$ of the world's total nuclear power generation [33]. Two types of HWRs were simulated in this study:

- The 28-element CANDU bundle employing natural uranium $\mathrm{UO}_{2}$ fuel

- The India-designed AHWR concept 


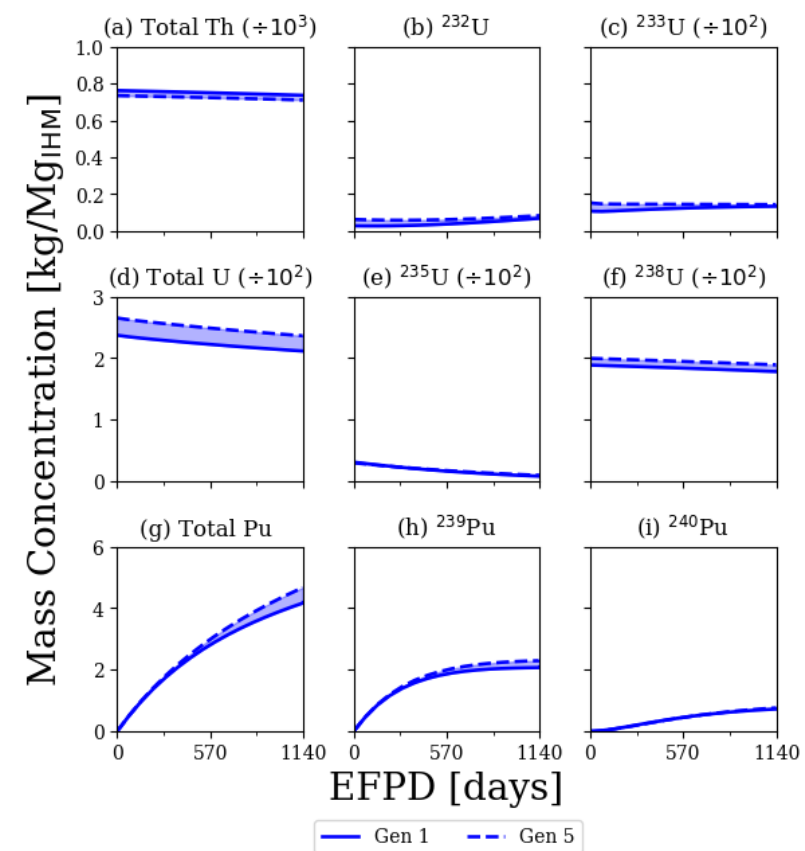

Figure 19. Th, $U$, and $\mathrm{Pu}$, mass concentrations vs. EFPDs for the homogeneous PWR fuel lattice with ${ }^{235} \mathrm{U}$ makeup for recycle Generations 1-5.

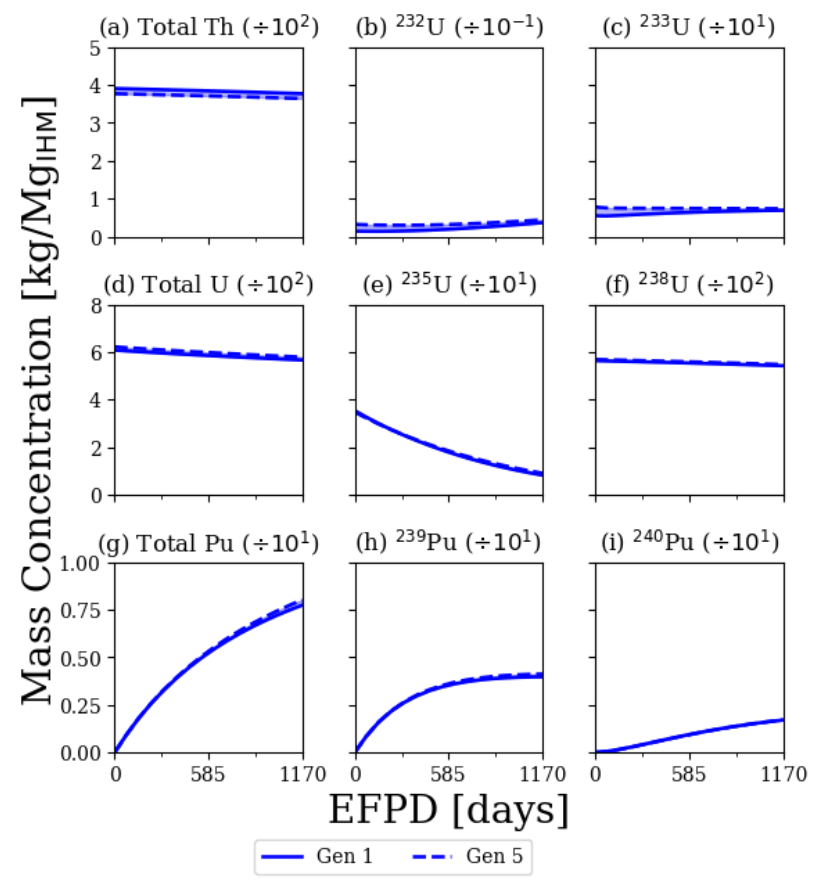

Figure 20. Th, $\mathrm{U}$, and $\mathrm{Pu}$, mass concentrations vs. EFPDs for the heterogeneous PWR fuel lattice with ${ }^{235} \mathrm{U}$ makeup and constant seed composition ( $4 \mathrm{wt} \%$ enriched $\mathrm{UO}_{2}$ ) for recycle Generations 1-5. 


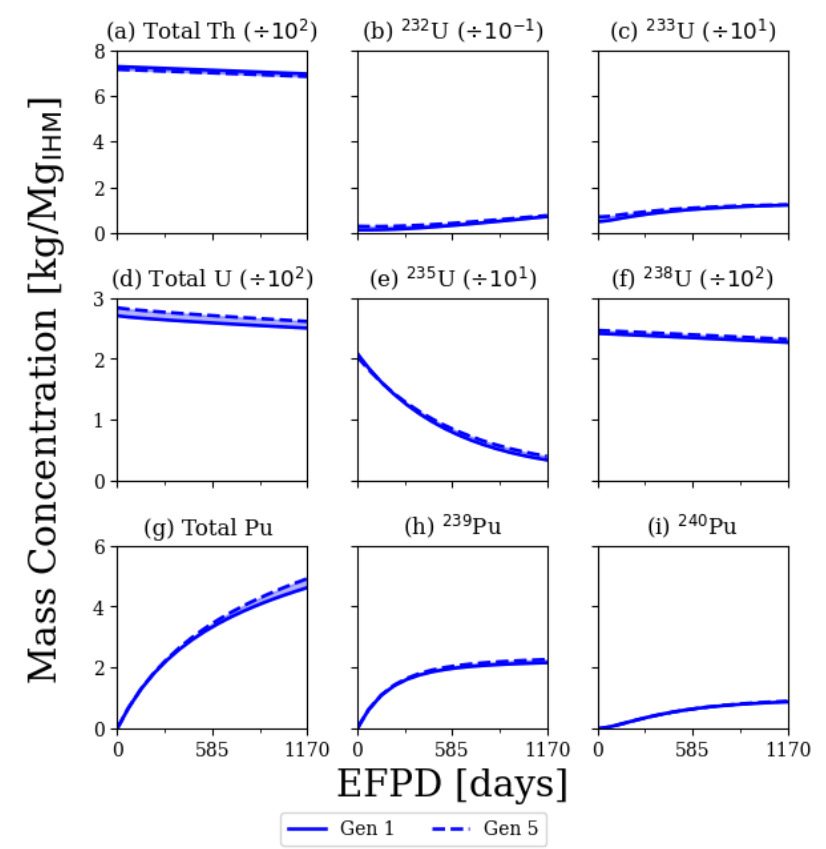

Figure 21. Th, $\mathrm{U}$, and $\mathrm{Pu}$, mass concentrations vs. EFPDs for the heterogeneous PWR fuel lattice with ${ }^{235} \mathrm{U}$ makeup and constant blanket composition ( $70 \% \mathrm{Th} / 30 \% \mathrm{U}$ ) for recycle Generations 1-5.

The 28-element CANDU bundle is composed of nonthorium-bearing HWR fuel that can be used as a basis for comparison of thorium fuels. The AHWR is a $300 \mathrm{MWe}$ vertical pressure tube-type reactor with heavy water as moderator and boiling light water as coolant [34]. The reactor uses reactor-grade $\mathrm{Pu}$-Th MOX, reactor-grade Pu-DU MOX, and ${ }^{233} \mathrm{U}-\mathrm{Th}$ MOX as its fuel. The AHWR is designed to produce most of its power from thorium ( $60 \%)$, with no external input of ${ }^{233} \mathrm{U}$ once the core design reaches its equilibrium configuration [21].

\subsubsection{28-Element Natural Uranium $\mathrm{UO}_{2}$-Fueled CANDU Bundle}

Figure 22 shows the 28-element CANDU lattice geometry. The bundle consists of three rings of zirconium-clad $\mathrm{UO}_{2}$ elements with a radius of $0.76 \mathrm{~cm}$ and an approximately triangular pitch of $1.6 \mathrm{~cm}$. CANDU reactors may employ other geometries, including those with 19 and 37 elements. Typically, CANDU reactors employ natural uranium, but low-enriched fuel can be used. The model was developed for another project, but it is used here as a basis for comparison for thorium-based fuels [9]. Table 14 shows the $\mathrm{UO}_{2}$ CANDU fuel lattice composition. 


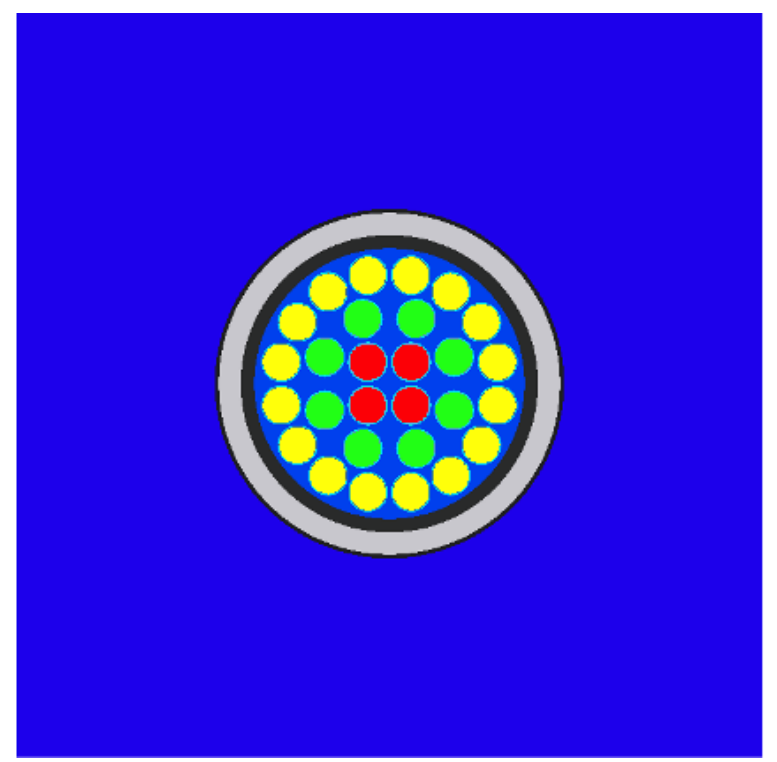

Figure 22. Natural uranium $\mathrm{UO}_{2}$ 28-element CANDU fuel lattice. Fuel elements are shown in three central rings (red, green, yellow), with interstitial light water coolant (blue). Concentric pressure and calandria tubes surround the bundle, with a heavy water moderator outside.

Table 14. $\mathrm{UO}_{2}$ CANDU fuel lattice composition.

\begin{tabular}{|c|c|c|c|c|c|c|c|c|c|c|c|c|}
\hline \multirow{2}{*}{ Fuel composition } & \multirow{2}{*}{$\begin{array}{c}\text { Th } \\
{ }^{232} \mathrm{Th}\end{array}$} & \multicolumn{6}{|c|}{$\mathbf{U}$} & \multicolumn{5}{|c|}{$\mathbf{P u}$} \\
\hline & & ${ }^{232} U$ & ${ }^{233} \mathrm{U}$ & ${ }^{234} U$ & ${ }^{235} \mathrm{U}$ & ${ }^{236} \mathrm{U}$ & ${ }^{238} \mathrm{U}$ & ${ }^{238} \mathrm{Pu}$ & ${ }^{239} \mathrm{Pu}$ & ${ }^{240} \mathrm{Pu}$ & ${ }^{241} \mathrm{Pu}$ & ${ }^{242} \mathrm{Pu}$ \\
\hline \multirow[t]{2}{*}{ Natural enriched } & $0 \%$ & \multicolumn{6}{|c|}{$100 \%$} & \multicolumn{5}{|c|}{$0 \%$} \\
\hline & $0 \%$ & $0 \%$ & $0 \%$ & $0.0063 \%$ & $0.711 \%$ & $0.0033 \%$ & $99.2794 \%$ & $0 \%$ & $0 \%$ & $0 \%$ & $0 \%$ & $0 \%$ \\
\hline
\end{tabular}

Figure 23 shows the resulting mass concentrations. Section 2.3.5 presents a comparison of ${ }^{233} \mathrm{U}$ and ${ }^{232} \mathrm{U}$ inventories across reactor types.

As of 2020, 22 nuclear power reactors operate in India, 2 of which are PWRs, 2 of which are boiling water reactors (BWRs), and the other 18 of which are PHWRs similar in design to CANDU reactors $[35,36]$. Because India has significant thorium reserves and most of its operating experience is with PHWRs, the country has focused on developing an AHWR optimized to use thorium fuels. The AHWR design has evolved over the last decade and a half, but many of its features have remained constant. The concept is a vertical pressure tube reactor, cooled by boiling light water, and moderated by heavy water contained in a stainless steel calandria [21]. Unlike CANDU fuel bundles that are $\sim 0.5 \mathrm{~m}$ in length, loaded horizontally, and refueled during reactor operation (i.e., online), AHWR fuel clusters are $3.5 \mathrm{~m}$ in length, loaded from the top of the core, and designed to be refueled offline $[37,38]$. 


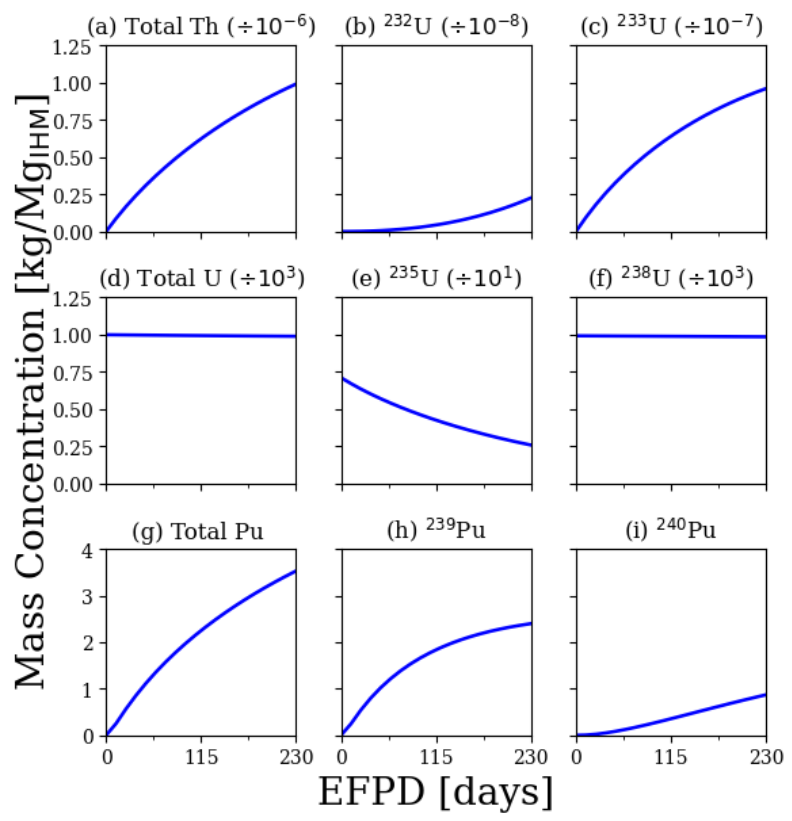

Figure 23. Th, $\mathrm{U}$, and $\mathrm{Pu}$, mass concentrations vs. EFPDs for the natural uranium $\mathrm{UO}_{2}$ 28-element CANDU fuel lattice.

\subsubsection{Advanced Heavy Water Reactor}

Because the AHWR is the leading candidate for a heavy water-moderated thorium-fueled reactor, the proposed fuel designs were simulated using data compiled from the literature, rather than parameterizing a range of possible designs as was done for the PWR thorium fuels. The models were assembled using dimensions and compositions presented in various references [38, 39]. According to Vijayan et al. [21], AHWRs will start up using an initial core consisting of one or two types of fuel clusters. The Initial Type I contains Th-Pu MOX (Table 15), and the Initial Type II contains DU-Pu MOX (Table 16). The AHWR is anticipated to eventually reach an equilibrium core where the reactor will have no net external fissile feed and the core will use a single fuel cluster type. The transition from the initial core to an equilibrium core will be a gradual process in which the fraction of ${ }^{233} \mathrm{U}$-Th MOX fuel clusters increases with time. During this period, the Transition Type of fuel cluster will be used that includes three rings of Th-Pu MOX of differing composition (Table 17). A transition using no external fissile feed is expected to take 20 years, but if ${ }^{233} \mathrm{U}$ feed from other reactor sources is employed, the transition could be as short as 5 years. The Equilibrium Type fuel cluster has inner and middle rings using Th-Pu MOX and an outer ring using [30] ${ }^{233} \mathrm{U}$-Th MOX (Table 18). Simulated fuel depletions to $20 \mathrm{GWd} / \mathrm{Mg}_{\mathrm{Hнм}}$ (the expected discharge burnup) for all four fuel cluster types were modeled. Figure 24 shows the AHWR fuel lattice.

Table 15. Fuel compositions of the AHWR Initial Type I fuel cluster.

\begin{tabular}{|l|c|c|c|c|c|c|c|c|c|c|c|c|}
\hline \multirow{2}{*}{ Fuel composition } & Th & \multicolumn{10}{c|}{$\mathbf{P u}$} \\
& ${ }^{232} \mathbf{T h}$ & ${ }^{232} \mathbf{U}$ & ${ }^{233} \mathbf{U}$ & ${ }^{234} \mathbf{U}$ & ${ }^{235} \mathbf{U}$ & ${ }^{236} \mathbf{U}$ & ${ }^{238} \mathbf{U}$ & ${ }^{238} \mathbf{P u}$ & ${ }^{239} \mathbf{P u}$ & ${ }^{240} \mathbf{P u}$ & ${ }^{241} \mathbf{P u}$ & ${ }^{242} \mathbf{P u}$ \\
\hline Initial Type I & $\mathbf{9 7 . 9 \%}$ & \multicolumn{8}{|c|}{$\mathbf{0 \%}$} & $\mathbf{2 . 1 \%}$ \\
& $100 \%$ & $0 \%$ & $0 \%$ & $0 \%$ & $0 \%$ & $0 \%$ & $0 \%$ & $0.095 \%$ & $67.990 \%$ & $24.586 \%$ & $6.005 \%$ & $1.324 \%$ \\
\hline
\end{tabular}


Table 16. Fuel compositions of the AHWR Initial Type II fuel cluster.

\begin{tabular}{|c|c|c|c|c|c|c|c|c|c|c|c|c|}
\hline \multirow{2}{*}{$\begin{array}{c}\text { Fuel } \\
\text { composition }\end{array}$} & \multicolumn{7}{|l|}{ Th } & \multicolumn{5}{|c|}{ Pu } \\
\hline & ${ }^{232} \mathrm{Th}$ & ${ }^{232} \mathrm{U}$ & ${ }^{233} \mathrm{U}$ & ${ }^{234} U$ & ${ }^{235} U$ & ${ }^{236} U$ & ${ }^{238} U$ & ${ }^{238} \mathrm{Pu}$ & ${ }^{239} \mathrm{Pu}$ & ${ }^{240} \mathrm{Pu}$ & ${ }^{241} \mathrm{Pu}$ & ${ }^{242} \mathrm{Pu}$ \\
\hline Initial Type & $0 \%$ & \multicolumn{6}{|c|}{$98.5 \%$} & \multicolumn{5}{|c|}{$1.5 \%$} \\
\hline II & $0 \%$ & $0 \%$ & $0 \%$ & $0.005 \%$ & $0.261 \%$ & $0.072 \%$ & $99.661 \%$ & $0.095 \%$ & $67.990 \%$ & $24.586 \%$ & $6.005 \%$ & $1.324 \%$ \\
\hline
\end{tabular}

Table 17. Fuel compositions of the AHWR Transition Type fuel cluster.

\begin{tabular}{|c|c|c|c|c|c|c|c|c|c|c|c|c|}
\hline \multirow{2}{*}{ Fuel composition } & \multirow{2}{*}{$\begin{array}{c}\text { Th } \\
{ }^{232} \mathrm{Th} \\
\end{array}$} & \multicolumn{6}{|c|}{$\mathbf{U}$} & \multicolumn{5}{|c|}{ Pu } \\
\hline & & ${ }^{232} U$ & ${ }^{233} \mathrm{U}$ & ${ }^{234} \mathrm{U}$ & ${ }^{235} \mathrm{U}$ & ${ }^{236} \mathrm{U}$ & ${ }^{238} \mathrm{U}$ & ${ }^{238} \mathrm{Pu}$ & ${ }^{239} \mathrm{Pu}$ & ${ }^{240} \mathrm{Pu}$ & ${ }^{241} \mathrm{Pu}$ & ${ }^{242} \mathrm{Pu}$ \\
\hline \multirow[t]{2}{*}{ Transition inner ring } & $91 \%$ & \multicolumn{6}{|c|}{$0 \%$} & \multicolumn{5}{|c|}{$9 \%$} \\
\hline & $100 \%$ & $0 \%$ & $0 \%$ & $0 \%$ & $0 \%$ & $0 \%$ & $0 \%$ & $0.095 \%$ & $67.990 \%$ & $24.586 \%$ & $6.005 \%$ & $1.324 \%$ \\
\hline \multirow[t]{2}{*}{ Transition middle ring } & $94.3 \%$ & \multicolumn{6}{|c|}{$0 \%$} & \multicolumn{5}{|c|}{$5.7 \%$} \\
\hline & $100 \%$ & $0 \%$ & $0 \%$ & $0 \%$ & $0 \%$ & $0 \%$ & $0 \%$ & $0.095 \%$ & $67.990 \%$ & $24.586 \%$ & $6.005 \%$ & $1.324 \%$ \\
\hline \multirow[t]{2}{*}{ Transition outer ring } & $97 \%$ & \multicolumn{6}{|c|}{$0 \%$} & \multicolumn{5}{|c|}{$3 \%$} \\
\hline & $100 \%$ & $0 \%$ & $0 \%$ & $0 \%$ & $0 \%$ & $0 \%$ & $0 \%$ & $0.095 \%$ & $67.990 \%$ & $24.586 \%$ & $6.005 \%$ & $1.324 \%$ \\
\hline
\end{tabular}

Table 18. Fuel compositions of the AHWR Equilibrium Type fuel cluster.

\begin{tabular}{|c|c|c|c|c|c|c|c|c|c|c|c|c|}
\hline \multirow{2}{*}{$\begin{array}{c}\text { Fuel } \\
\text { Composition }\end{array}$} & Th & \multicolumn{6}{|c|}{$\mathbf{U}$} & \multicolumn{5}{|c|}{$\mathbf{P u}$} \\
\hline & ${ }^{232} \mathrm{Th}$ & ${ }^{232} \mathrm{U}$ & ${ }^{233} \mathrm{U}$ & ${ }^{234} \mathrm{U}$ & ${ }^{235} \mathrm{U}$ & ${ }^{236} \mathrm{U}$ & ${ }^{233} \mathrm{U}$ & ${ }^{238} \mathrm{Pu}$ & ${ }^{239} \mathrm{Pu}$ & ${ }^{240} \mathrm{Pu}$ & ${ }^{241} \mathrm{Pu}$ & ${ }^{242} \mathrm{Pu}$ \\
\hline \multirow{2}{*}{$\begin{array}{l}\text { Equilibrium } \\
\text { inner ring }\end{array}$} & $94 \%$ & \multicolumn{6}{|c|}{$0 \%$} & \multicolumn{5}{|c|}{$6 \%$} \\
\hline & $100 \%$ & $0 \%$ & $0 \%$ & $0 \%$ & $0 \%$ & $0 \%$ & $0 \%$ & $0.095 \%$ & $67.990 \%$ & $24.586 \%$ & $6.005 \%$ & $1.324 \%$ \\
\hline \multirow{2}{*}{$\begin{array}{l}\text { Equilibrium } \\
\text { middle ring }\end{array}$} & $96.1 \%$ & \multicolumn{6}{|c|}{$0 \%$} & \multicolumn{5}{|c|}{$3.9 \%$} \\
\hline & $100 \%$ & $0 \%$ & $0 \%$ & $0 \%$ & $0 \%$ & $0 \%$ & $0 \%$ & 0.095\% & $67.990 \%$ & $24.586 \%$ & $6.005 \%$ & $1.324 \%$ \\
\hline \multirow{2}{*}{$\begin{array}{l}\text { Equilibrium } \\
\text { outer ring }\end{array}$} & $96.5 \%$ & \multicolumn{6}{|c|}{$3.5 \%$} & \multicolumn{5}{|c|}{$0 \%$} \\
\hline & $100 \%$ & $0.0323 \%$ & $95.627 \%$ & $4.2364 \%$ & $0.1015 \%$ & $0.0023 \%$ & $0 \%$ & $0 \%$ & $0 \%$ & $0 \%$ & $0 \%$ & $0 \%$ \\
\hline
\end{tabular}

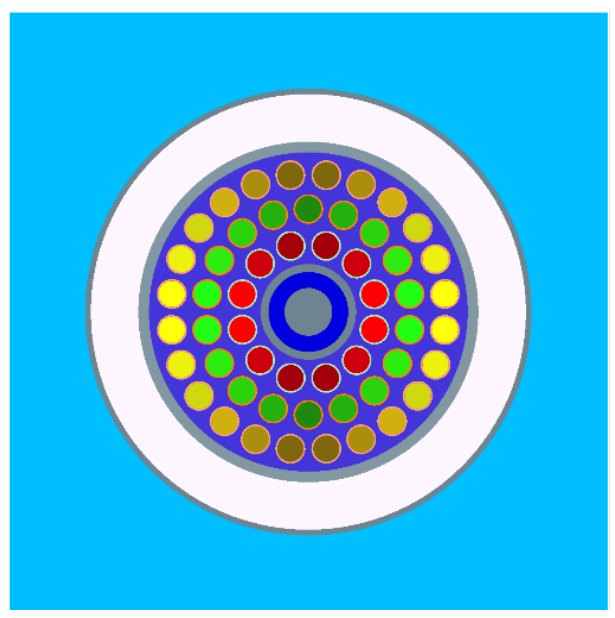

Figure 24. AHWR fuel lattice. A light water coolant channel with a displacer occupies the central location. Fuel elements are shown in three central rings (red, green, yellow), with interstitial light water coolant (purple). Concentric pressure and calandria tubes surround the bundle, with a heavy water moderator outside (light blue). 
Figures $25-28$ show the resulting mass concentrations. Section 2.3.5 presents a comparison of ${ }^{233} \mathrm{U}$ and ${ }^{232} \mathrm{U}$ inventories across reactor types.

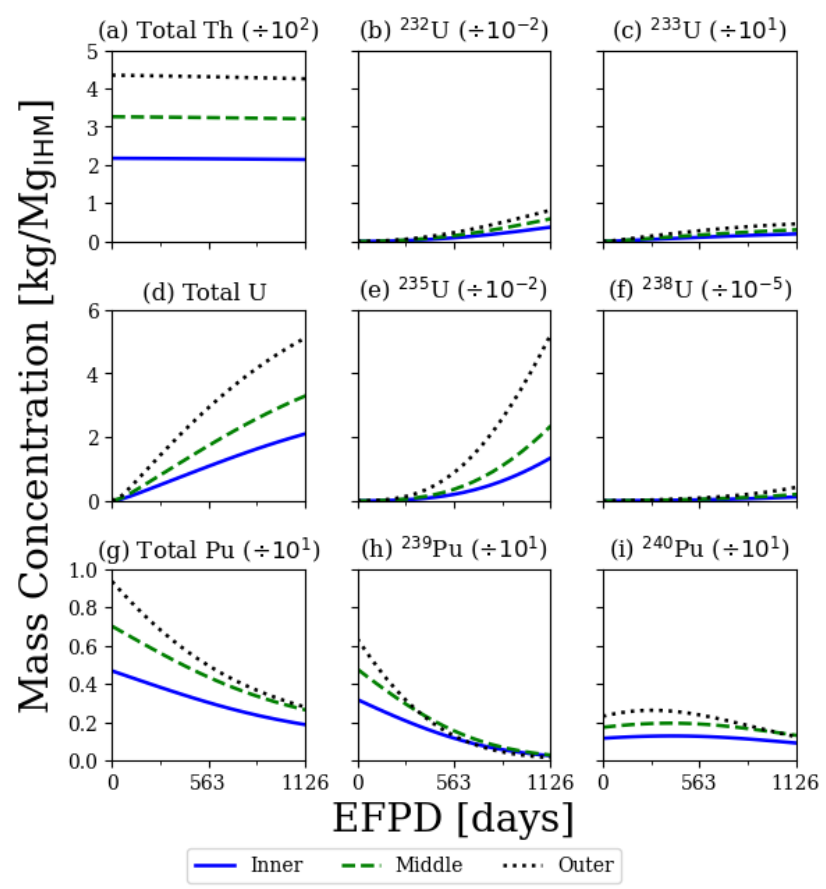

Figure 25. Th, $\mathrm{U}$, and $\mathrm{Pu}$, mass concentrations vs. EFPDs for the AHWR Initial Type I fuel cluster showing composition of the inner, middle, and outer rings.

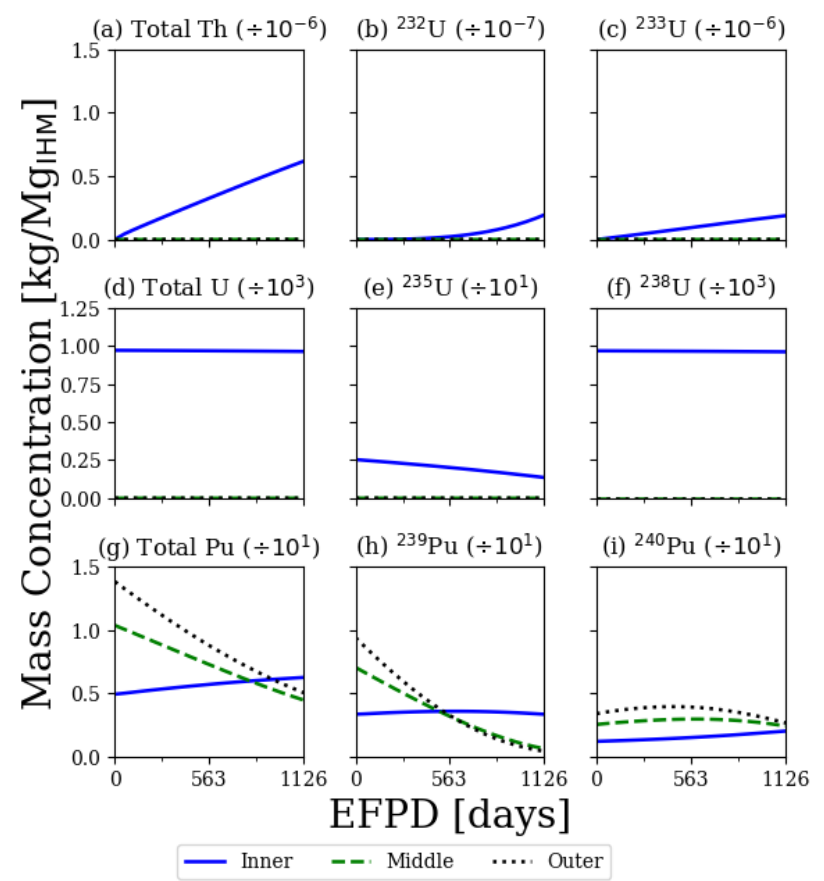

Figure 26. Th, $U$, and Pu, mass concentrations vs. EFPDs for the AHWR Initial Type II fuel cluster showing composition of the inner, middle, and outer rings. 


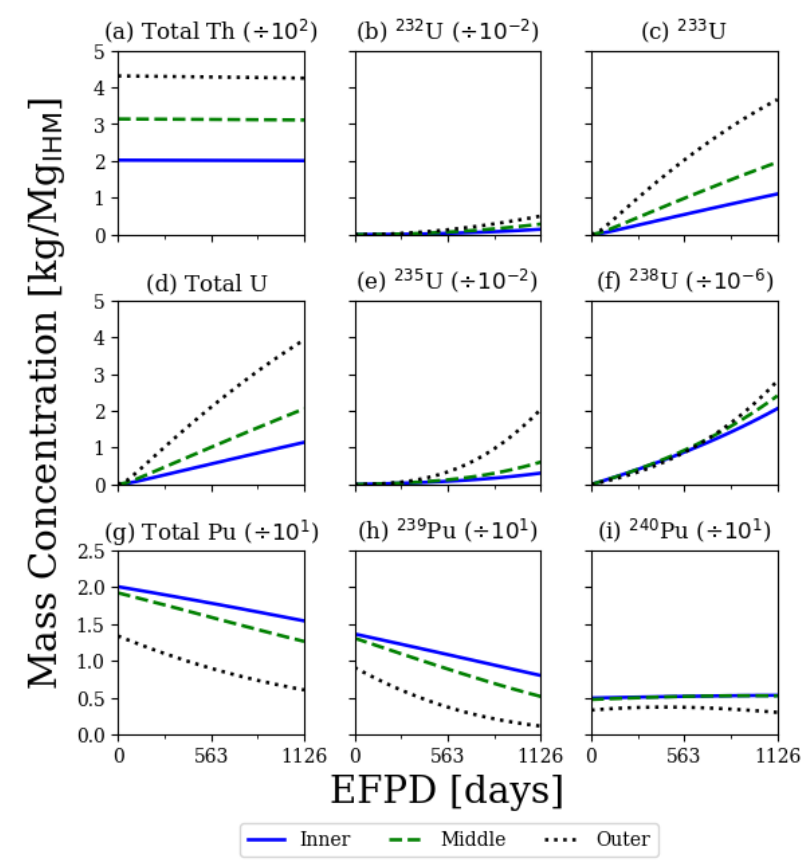

Figure 27. Th, $U$, and Pu, mass concentrations vs. EFPDs for the AHWR Transition fuel cluster showing composition of the inner, middle, and outer rings.

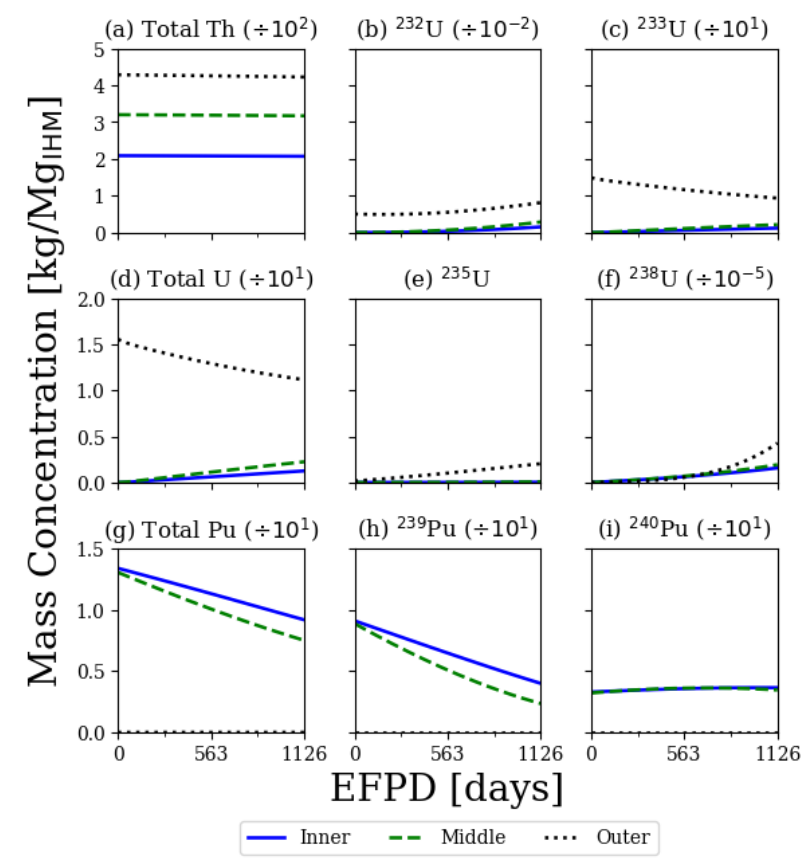

Figure 28. Th, $\mathrm{U}$, and Pu, mass concentrations vs. EFPDs for the AHWR Equilibrium fuel cluster showing composition of the inner, middle, and outer rings. 


\subsubsection{Sodium-cooled Fast Breeder Reactor with a Thorium Breeding Blanket}

India has adopted a three-stage nuclear power program to utilize its large thorium reserves. The first stage of the country's strategy involves using natural uranium to fuel PHWRs. The SNF is then reprocessed to extract "reactor-grade" plutonium $\left(<80 \%{ }^{239} \mathrm{Pu}\right)$. In the second stage, the "reactorgrade" plutonium is used as fuel for FBRs. The FBR core blanket can be either DU or thorium. The DU blanket produces additional plutonium, while the thorium blanket produces ${ }^{233} \mathrm{U}$. The additional plutonium can be used as fuel for these second-stage FBRs, while ${ }^{233} \mathrm{U}$ is the fuel for the third-stage breeder reactors, making the transition to a ${ }^{233} \mathrm{U}$-Th fuel cycle during this third stage. For this work, the thorium blanket was simulated.

The prototype fast breeder reactor (PFBR) is the proposed design for the second stage of India's power program. The PFBR is a $1250 \mathrm{MWt}(500 \mathrm{MWe})$ sodium-cooled reactor. It uses MOX fuel $\left(\mathrm{PuO}_{2} / \mathrm{UO}_{2}\right)$ in the core region and depleted $\mathrm{UO}_{2}$ or thorium in the radial and axial blanket regions. The core has two enrichment zones for power flattening. The inner zone consists of 85 fuel assemblies with an enrichment of $20.7 \mathrm{wt} \%{ }^{235} \mathrm{U}$. The outer zone has 96 assemblies with an enrichment of $27.7 \mathrm{wt} \%{ }^{235} \mathrm{U}[21,40-42]$. Each fuel subassembly consists of 217 helium-bonded fuel pins. The active fuel length is $100 \mathrm{~cm}$ with two $30 \mathrm{~cm}$ axial blankets above and below the fuel region. The radial blanket consists of 120 assemblies. Detailed fuel design parameters are summarized in Table 19. The core inventory is given in Table 20.

Based on the dimensions in Table 19, the total fuel volume is $8.76 \times 10^{5} \mathrm{~cm}^{3}$. The mass density of MOX fuel is calculated as $10.44 \mathrm{~g} / \mathrm{cm}^{3}$ based on the total fuel inventory in the core. The uranium fuel isotopic composition is $0.0055 \%{ }^{234} \mathrm{U}, 0.7203 \%{ }^{235} \mathrm{U}$, and the balance ${ }^{238} \mathrm{U}$ [40]. The weight fractions of plutonium in the fuel isotopic composition in Table 21 are obtained from CANDU fuel burnup at 6.7 MWd/kg. From Table 21, the ${ }^{239} \mathrm{Pu}$ weight percent is $67.99 \%$. From Ref. [21] data, the ${ }^{239} \mathrm{Pu}$ weight percent is $67.99 \%$. Thus, the estimated ${ }^{235} \mathrm{U}$ enrichment and ${ }^{239} \mathrm{Pu}$ weight percent from the data collected in the fast reactor database agree with the data from CANDU fuel burnup at $6.7 \mathrm{MWd} / \mathrm{kg}$.

Table 19. PFBR fuel design parameters.

\begin{tabular}{|l|c|}
\hline \multicolumn{1}{|c|}{ Item } & PFBR \\
\hline Number of fuel pins per assembly - core & 217 \\
\hline Number of fuel pins per assembly - blanket & 61 \\
\hline Core fuel pin outer diameter (mm) & 6.6 \\
\hline Core fuel pin cladding thickness (mm) & 0.45 \\
\hline Active fuel length (mm) & 1000 \\
\hline Axial blanket length above and below the fuel region $(\mathrm{mm})$ & 300 \\
\hline Core fuel pin overall length (mm) & 2580 \\
\hline Blanket fuel pin outer diameter (mm) & 14.33 \\
\hline Blanket fuel pin cladding thickness (mm) & 0.6 \\
\hline Blanket fuel pin overall length (mm) & 1960 \\
\hline
\end{tabular}


Table 20. PFBR core characteristics.

\begin{tabular}{|l|c|}
\hline \multicolumn{1}{|c|}{ Item } & PFBR \\
\hline Fissile material content of a core $(\mathrm{kg})-{ }^{235} \mathrm{U}$ & 17.3 \\
\hline Fissile material content of a core $(\mathrm{kg})-{ }^{239} \mathrm{Pu}$ & 1361 \\
\hline Fissile material content of a core $(\mathrm{kg})$ - total plutonium & 1978 \\
\hline Fuel volume fraction averaged over whole core & 0.297 \\
\hline Sodium volume fraction averaged over whole core & 0.410 \\
\hline Fuel $\mathrm{PuO}_{2}-\mathrm{UO}_{2}$ inventory in active core (ton) & 9.15 \\
\hline
\end{tabular}

Table 21. Fuel compositions of the PFBR.

\begin{tabular}{|c|c|c|c|c|c|c|c|c|c|c|c|c|}
\hline \multirow{2}{*}{$\begin{array}{c}\text { Fuel } \\
\text { composition }\end{array}$} & \multirow{2}{*}{$\begin{array}{c}\text { Th } \\
{ }^{232} \mathrm{Th}\end{array}$} & \multicolumn{6}{|c|}{$\mathbf{U}$} & \multicolumn{5}{|c|}{$\mathrm{Pu}$} \\
\hline & & ${ }^{232} \mathrm{U}$ & ${ }^{233} U$ & ${ }^{234} \mathrm{U}$ & ${ }^{235} \mathrm{U}$ & ${ }^{236} \mathrm{U}$ & ${ }^{238} \mathrm{U}$ & ${ }^{238} \mathrm{Pu}$ & ${ }^{239} \mathrm{Pu}$ & ${ }^{240} \mathrm{Pu}$ & ${ }^{241} \mathrm{Pu}$ & ${ }^{242} \mathrm{Pu}$ \\
\hline \multirow[t]{2}{*}{ Inner driver } & $0 \%$ & \multicolumn{6}{|c|}{$79.3 \%$} & \multicolumn{5}{|c|}{$20.7 \%$} \\
\hline & $0 \%$ & $0 \%$ & $0 \%$ & $0.0055 \%$ & $0.72 \%$ & $0 \%$ & $99.27 \%$ & $0.0945 \%$ & $67.99 \%$ & $24.59 \%$ & $6.01 \%$ & $1.32 \%$ \\
\hline \multirow[t]{2}{*}{ Outer driver } & $0 \%$ & \multicolumn{6}{|c|}{$72.3 \%$} & \multicolumn{5}{|c|}{$27.7 \%$} \\
\hline & $0 \%$ & $0 \%$ & $0 \%$ & $0.0055 \%$ & $0.72 \%$ & $0 \%$ & $99.27 \%$ & $0.0945 \%$ & $67.99 \%$ & $24.59 \%$ & $6.01 \%$ & $1.32 \%$ \\
\hline \multirow[t]{2}{*}{ Blanket } & $100 \%$ & \multicolumn{6}{|c|}{$0 \%$} & \multicolumn{5}{|c|}{$0 \%$} \\
\hline & $100 \%$ & $0 \%$ & $0 \%$ & $0 \%$ & $0 \%$ & $0 \%$ & $0 \%$ & $0 \%$ & $0 \%$ & $0 \%$ & $0 \%$ & $0 \%$ \\
\hline
\end{tabular}

The simulation was carried out using the TRITON (Transport Rigor Implemented with Time-dependent Operation for Neutronic Depletion) depletion sequences t6-dep/ within the SCALE 6.2.3 code package [9]. Given the power density of $1247 \mathrm{~kW} /$ liter from Ref. [20], the average specific power was estimated as 141.4 megawatts per metric tonne of initial heavy metal. The cycle length of 240 EFPDs from Ref. [40] was adopted in calculations.

Figure 29 provides a radial view of the PFBR core geometry of a slice through the middle of the fuel assemblies. Figure 30 provides an axial and radial view of fuel assembly geometry.
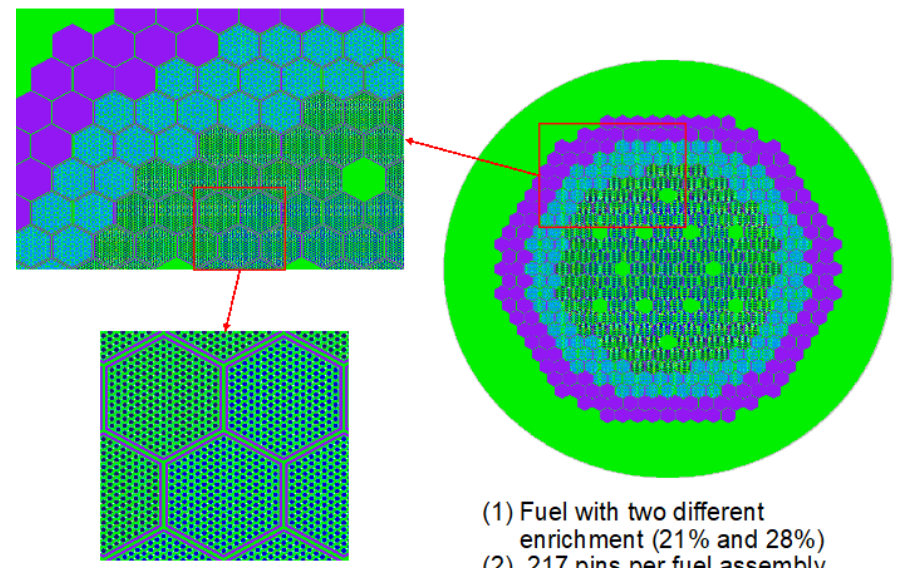

(1) Fuel with two different

enrichment $(21 \%$ and $28 \%)$

(2) 217 pins per fuel assembly

Figure 29. PFBR core configuration (X-Y 2D view). The green is molten sodium, the purple is stainless steel, the light blue is the radial blanket, and the dark blue are the inner/outer fuel. 


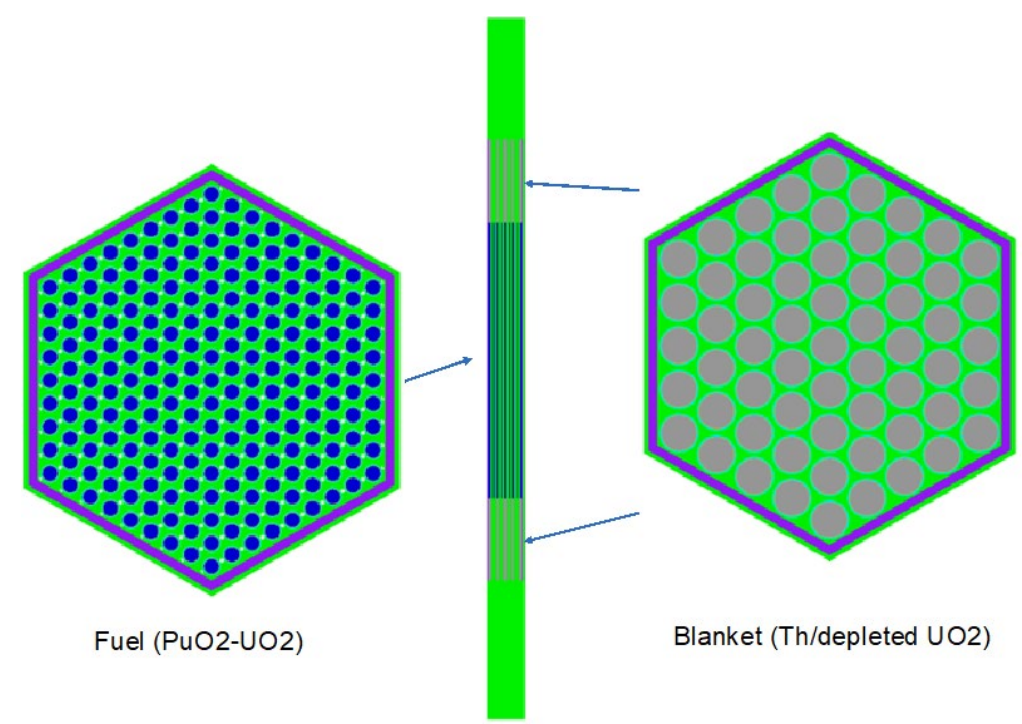

Figure 30. PFBR fuel assembly. The green is molten sodium, the gray is the axial blanket, the purple is stainless steel, and the dark blue is the fuel.

The objective of the simulations was to obtain the inventory data of fuel as well as the axial and radial blankets as a function of burnup. The ${ }^{239} \mathrm{Pu}$ production and ${ }^{233} \mathrm{U}$ production can be derived from the data. In this preliminary investigation, the core was burned with a fixed power density for a full cycle length. Equilibrium conditions, fuel shuffling scheme, and movement of control rods and safety rods were not taken into consideration.

Figure 31 shows the inventories in the driver fuel for select isotopes, while the isotopic inventories in the axial and radial blankets are shown in Figures 32 and 33, respectively. From these figures, roughly $185 \mathrm{~kg}$ of uranium and $97 \mathrm{~kg}$ of plutonium were burned in a full cycle. This included $114 \mathrm{~kg}(3.9 \%)$ of uranium and $34.7 \mathrm{~kg}$ (4.6\%) of the plutonium from the inner core region and $71.7 \mathrm{~kg}(2.4 \%)$ of uranium and $62.2 \mathrm{~kg}(5.4 \%)$ of plutonium from the outer core region. Section 2.3 .5 compares $^{233} \mathrm{U}$ and ${ }^{232} \mathrm{U}$ inventories across reactor types.

Scaling the results from Figures 31-33 to the $26.4 \mathrm{Mg}$ of initial heavy metal that was used in the model shows that $\sim 92 \mathrm{~kg}$ of ${ }^{233} \mathrm{U}$ is generated from one full-cycle irradiation. This includes $51 \mathrm{~kg}$ of ${ }^{233} \mathrm{U}$ from the radial blanket and $41 \mathrm{~kg}$ from the axial blanket. These results are based on a single fuel cycle that is 240 EFPDs; however, from the IAEA Fast Reactor Database [40], a fuel assembly can stay in the core for a maximum of three cycles with a refueling scheme. The radial blanket can stay in the core for up to seven cycles depending on proximity to the core. In future work, equilibrium conditions, fuel shuffling scheme, and movement of control rods and safety rods should be taken into consideration for a more accurate estimation of ${ }^{233} \mathrm{U}$ production. 


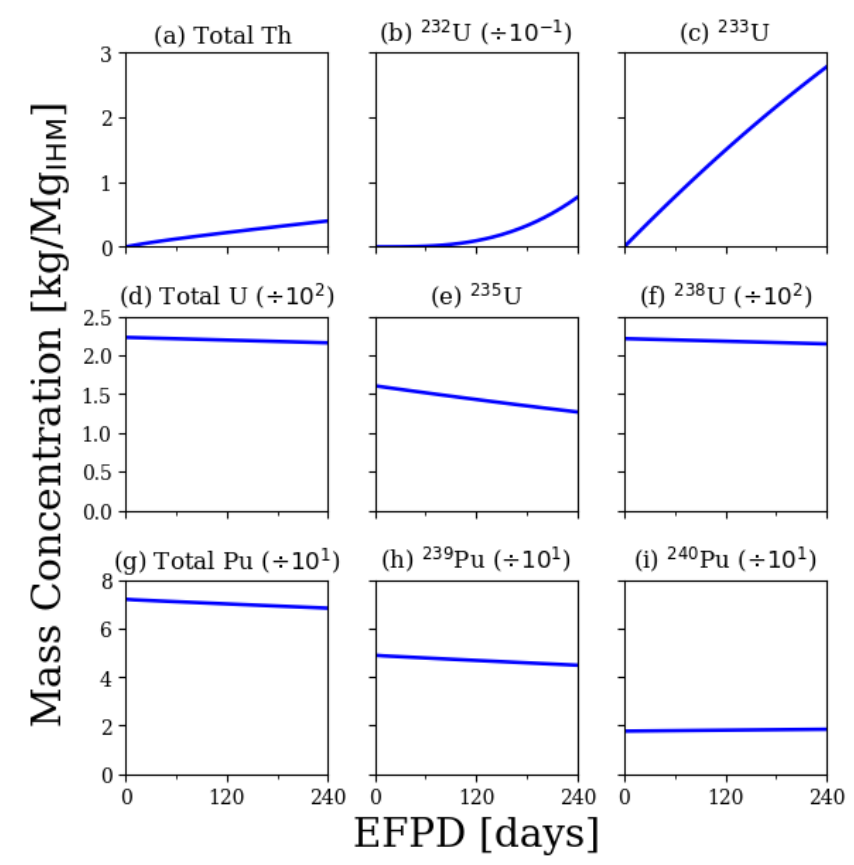

Figure 31. Th, $\mathrm{U}$, and Pu, mass concentrations vs. EFPDs for the PFBR driver fuel.

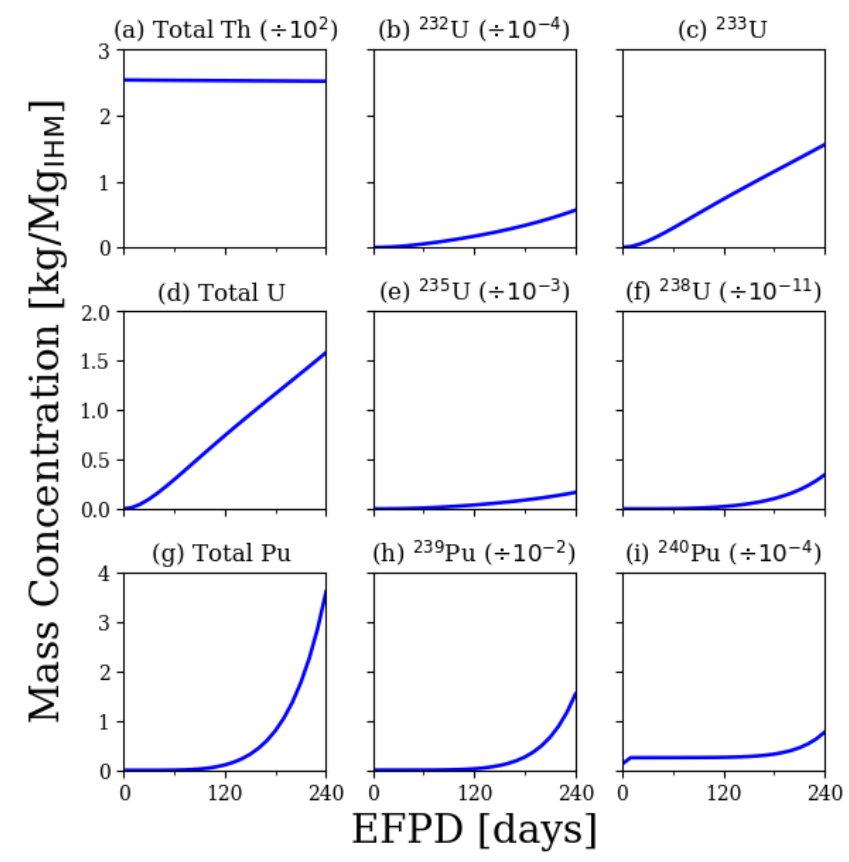

Figure 32. Th, $\mathrm{U}$, and Pu, mass concentrations vs. EFPDs for the PFBR axial blanket. 


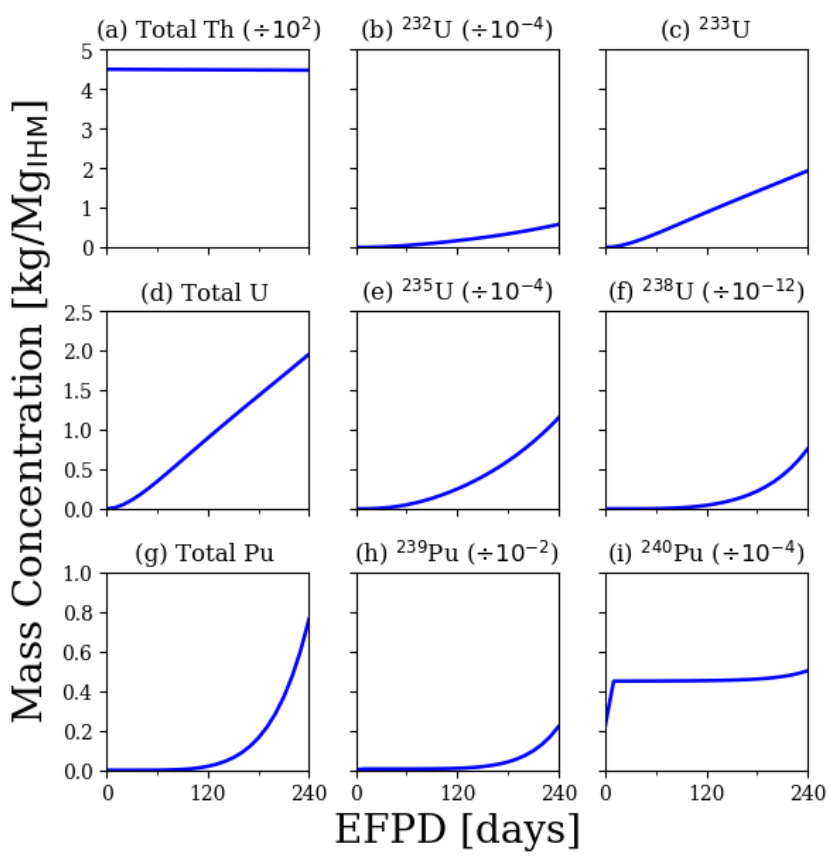

Figure 33. Th, $\mathrm{U}$, and $\mathrm{Pu}$, mass concentrations vs. EFPDs for the PFBR radial blanket.

\subsubsection{Molten Salt Reactor}

A model for a thorium-fueled MSR concept was created for another project [43, 44], and typical isotope inventories derived from those simulations are presented in this section. The molten salt breeder reactor (MSBR) prototype was a thermal spectrum reactor designed by ORNL to operate on the Th- ${ }^{233} \mathrm{U}$ fuel cycle using a $\mathrm{LiF}_{-} \mathrm{BeF}_{2}-\mathrm{ThF}_{4}-\mathrm{UF}_{4}$ salt [45]. The breeding ratio of the reactor was to be 1.06 , with an annual fissile yield of $3.2 \%$ and a doubling time of 22 years. Fission products, including metals and noble gases, would be removed from the salt with continuous online chemical processing systems. Isotopes of protactinium, most importantly ${ }^{233} \mathrm{~Pa}$ and ${ }^{232} \mathrm{~Pa}$, would also be removed from the salt and allowed to decay in isolation tanks to ${ }^{233} \mathrm{U}$ and ${ }^{232} \mathrm{U}$, respectively. Fertile thorium (primarily ${ }^{232} \mathrm{Th}$ ) and fissile uranium (primarily ${ }^{233} \mathrm{U}$ ) would be fed back into the primary salt loop at a rate that would maintain equilibrium reactor power operations.

The initial fuel composition used in the MSBR model is shown in Table 22. The MSBR concept uses a single salt, but it employs multiple zones of differing cell geometry to promote both power production from fission and breeding from fertile nuclide neutron capture. The model used to simulate the MSBR considers a single unit cell that was determined to be equivalent to the multiple zones (see Figure 34). The simulation includes continuous feed of thorium and uranium along with continuous removal of fission products and protactinium. Figure 35 shows the evolution of the primary thorium, uranium, and plutonium isotopes during depletion. Section 2.3.5 presents a comparison of ${ }^{233} \mathrm{U}$ and ${ }^{232} \mathrm{U}$ inventories across reactor types. 
Table 22. Initial fuel compositions of the MSBR fuel salt.

\begin{tabular}{|l|c|c|c|c|c|c|c|c|c|c|c|c|}
\hline \multirow{2}{*}{ Fuel composition } & Th & \multicolumn{10}{c|}{$\mathbf{P}$ u } \\
& ${ }^{232} \mathbf{T h}$ & ${ }^{232} \mathbf{U}$ & ${ }^{233} \mathbf{U}$ & ${ }^{234} \mathbf{U}$ & ${ }^{235} \mathbf{U}$ & ${ }^{236} \mathbf{U}$ & ${ }^{238} \mathbf{U}$ & ${ }^{238} \mathbf{P u}$ & ${ }^{239} \mathbf{P u}$ & ${ }^{240} \mathbf{P u}$ & ${ }^{241} \mathbf{P u}$ & ${ }^{242} \mathbf{P u}$ \\
\hline Fuel salt & $\mathbf{9 8 . 3 5 \%}$ & \multicolumn{8}{|c|}{$\mathbf{1 . 6 4 6 \%}$} & $\mathbf{0 \%}$ & \\
& $100 \%$ & $0 \%$ & $100 \%$ & $0 \%$ & $0 \%$ & $0 \%$ & $0 \%$ & $0 \%$ & $0 \%$ & $0 \%$ & $0 \%$ & $0 \%$ \\
\hline
\end{tabular}

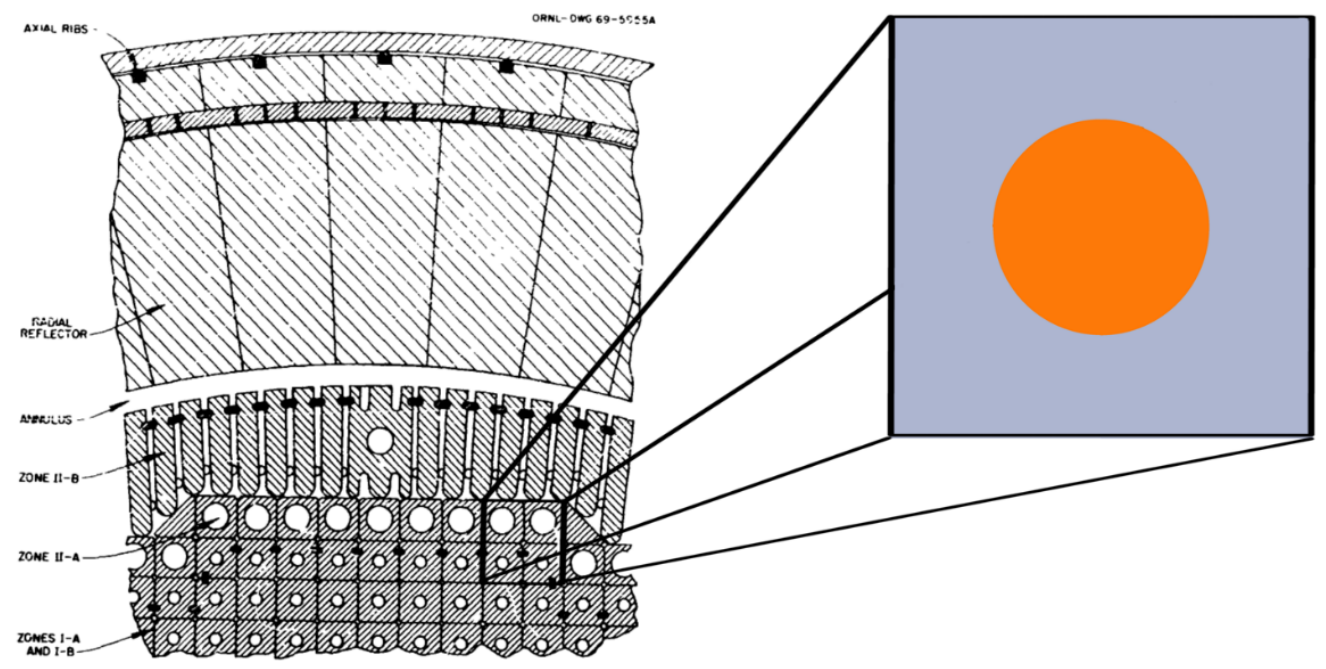

Figure 34. Core design of the MSBR showing the multiple zones optimized for power production (from [33]). Inset shows the geometry of the equivalent unit cell used in the MSBR model. The gray is graphite and the orange is the fuel salt.

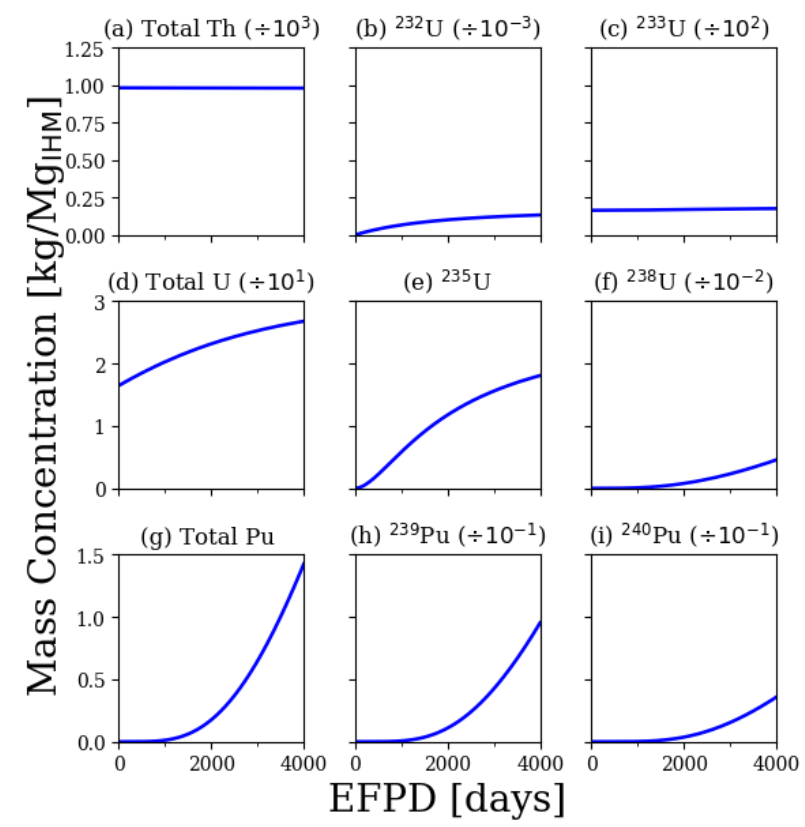

Figure 35. Th, U, Pu, mass concentrations vs. EFPDs for the MSBR fuel salt. 


\subsubsection{Comparison of ${ }^{233} \mathrm{U}$ and ${ }^{232} \mathrm{U}$ Inventories Across Reactor Types}

The ${ }^{233} \mathrm{U}$ and ${ }^{232} \mathrm{U}$ inventories of the SNF from the leading thorium fuel cycles can be compared across reactor type and fuel design. Figure 36 shows the grams of ${ }^{233} \mathrm{U}$ present in the fuel per kilogram of initial thorium. Shaded bands are shown for the parameterized PWR fuel designs that bound the range of values, while traces are shown for the fixed designs. In a reactor, the burnup of any single region of the fuel (e.g., pin, assembly, or zone) will usually differ from the burnup of the entire system.

Therefore, the data are plotted against an $\mathrm{x}$-axis of system burnup, which considers all fuel materials in the model. While the data for the PWR and MSR systems extend beyond the $30 \mathrm{GWd} / \mathrm{Mg}_{\text {Iнм }}$ upper bound of the plot, the systems' behavior continues the trends shown in the plot, with the MSR and the PWR Recycle ${ }^{235} \mathrm{U}$ bands remaining relatively flat, the PWR Recycle ${ }^{233} \mathrm{U}$ band decreasing monotonically, and the PWR Hetero 90\% U and PWR Recycle Blanket bands increasing monotonically. The traces for the AHWR and PFBR fuel end at their expected discharge burnups.

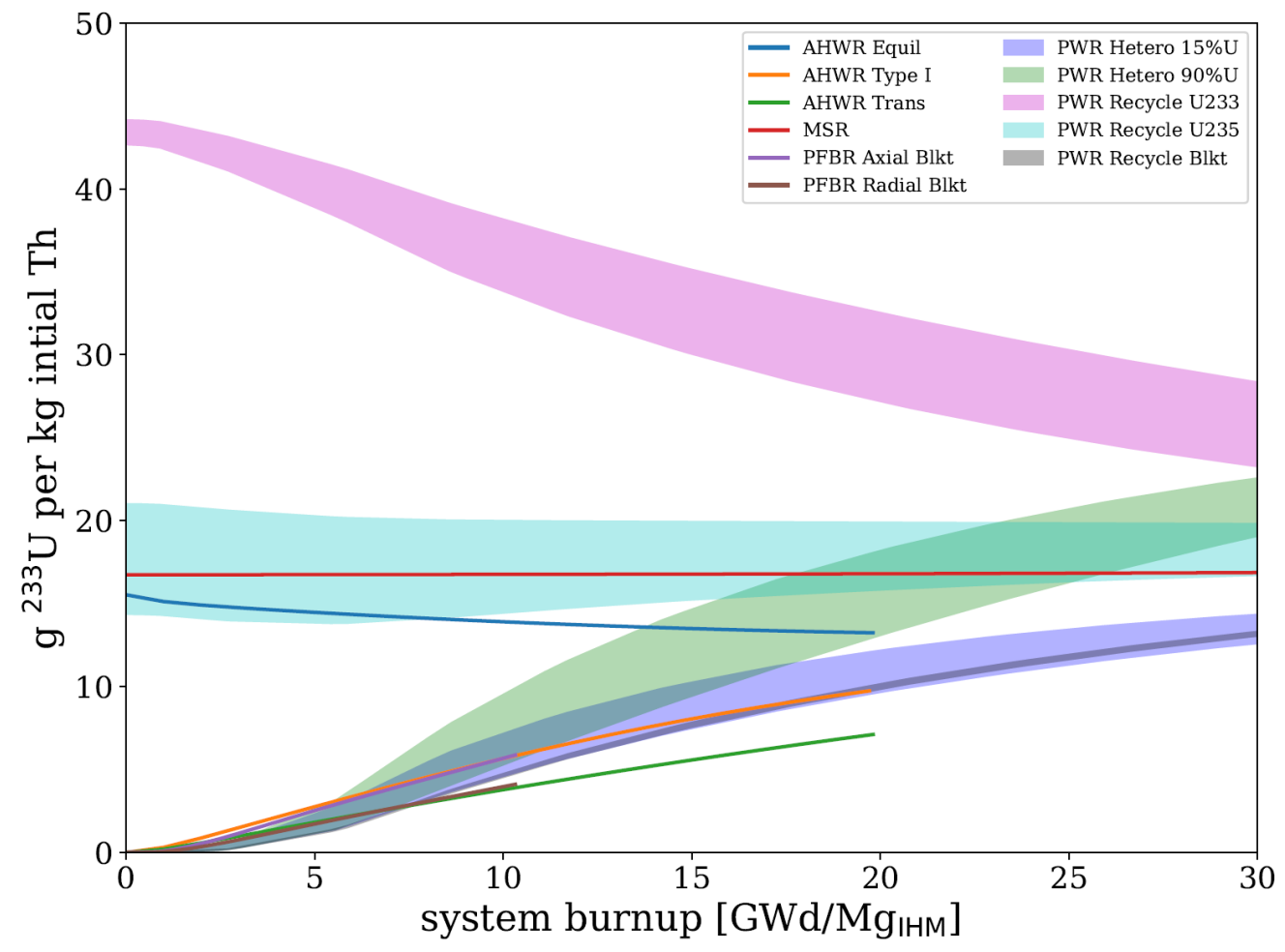

Figure 36. Comparison across reactor types of the mass concentration of ${ }^{233} \mathrm{U}$ per initial thorium.

For initial-use thorium fuels with no ${ }^{233} \mathrm{U}$ present in the fresh fuel, Figure 36 shows that the expected mass of ${ }^{233} \mathrm{U}$ at discharge ranges of $0-25 \mathrm{~g}^{233} \mathrm{U}$ per $\mathrm{kg}$ initial thorium depends on discharge burnup, regardless of reactor type. For the three fuel designs with ${ }^{233} \mathrm{U}$ present in the fresh fuel (i.e., MSR, PWR Recycle ${ }^{235} \mathrm{U}$, and AHWR Equilibrium), the expected mass of ${ }^{233} \mathrm{U}$ at discharge ranges remains relatively constant, from $15 \mathrm{~g}^{233} \mathrm{U}$ to $21 \mathrm{~g}^{233} \mathrm{U}$ per $\mathrm{kg}$ initial thorium. The mass of ${ }^{233} \mathrm{U}$ in the fresh fuel of the PWR Recycle with ${ }^{233} \mathrm{U}$ makeup is significantly greater than the other fuel types at $\sim 43 \mathrm{~g}{ }^{233} \mathrm{U}$ per $\mathrm{kg}$ initial thorium, but over the course of the $30 \mathrm{GWd} / \mathrm{Mg}_{\text {Інм }}$ depletion, this begins to approach the mass concentrations of the other fuel types with less than $30 \mathrm{~g}{ }^{233} \mathrm{U}$ per $\mathrm{kg}$ initial thorium. The conclusion that can be drawn from Figure 36 is that, regardless of reactor type, the mass concentration at discharge of ${ }^{233} \mathrm{U}$ will range from $5 \mathrm{~g}^{233} \mathrm{U}$ to $30 \mathrm{~g}{ }^{233} \mathrm{U}$ per kg initial thorium, with a typical value of $\sim 20 \mathrm{~g}$ 
${ }^{233} \mathrm{U}$ per $\mathrm{kg}$ initial thorium. This is equivalent to $\sim 3.5 \mathrm{~kg}{ }^{233} \mathrm{U}$ per SNF assembly as well as the equivalent fuel salt loading for MSR concepts. Table 23 provides the estimated masses of ${ }^{233} \mathrm{U}$ per initial thorium mass in each assembly type at discharge. As an example, for an approximate initial loading of $191 \mathrm{~kg}$ of thorium, based on a 15\% uranium PWR fuel assembly, $2.56 \mathrm{~kg}$ of ${ }^{233} \mathrm{U}$ would be present in each fuel assembly. In the MSR case, this is $0.0077 \mathrm{~kg}^{233} \mathrm{U}$ per $\mathrm{kg}$ of fuel salt.

Table 23. Estimated masses of ${ }^{233} \mathrm{U}$ per initial thorium mass in assembly types at discharge.

\begin{tabular}{|l|c|c|c|}
\multicolumn{1}{|c|}{ Assembly Type } & $\mathbf{M g}_{\text {Iнм }}$ & Initial Th (Mg) & Discharge ${ }^{233} \mathbf{U}(\mathbf{k g})$ \\
\hline AHWR Equilibrium & 0.118 & 0.113 & 1.49 \\
\hline AHWR Type I & 0.118 & 0.115 & 1.12 \\
\hline AHWR Transition & 0.118 & 0.112 & 0.79 \\
\hline PFBR Axial Blanket & 0.037 & 0.037 & 0.22 \\
\hline PFBR Radial Blanket & 0.099 & 0.099 & 0.41 \\
\hline PWR Hetero 15\% U & 0.441 & 0.191 & 2.56 \\
\hline PWR Hetero 90\% U & 0.458 & 0.024 & 0.50 \\
\hline PWR Recycle ${ }^{233} \mathrm{U}$ & 0.354 & 0.206 & 5.42 \\
\hline PWR Recycle ${ }^{235} \mathrm{U}$ & 0.353 & 0.212 & 3.85 \\
\hline PWR Recycle Blanket & 0.229 & 0.160 & 2.10 \\
\hline
\end{tabular}

The ${ }^{232} \mathrm{U}$ decay products contribute distinguishing features to the gamma ray spectrum, which is important for the detection, characterization, and monitoring of thorium fuels. The atom concentration of ${ }^{232} \mathrm{U}$ per total uranium in units of parts per million (ppm) is shown in Figure 37. Figure 37 shows that, unlike the ${ }^{233} \mathrm{U}$ mass concentration shown in Figure 36, the ${ }^{232} \mathrm{U}$ concentration spans several orders of magnitude depending on fuel type. With the exceptions of the cases of PWR Recycle fuel with ${ }^{233} \mathrm{U}$ and ${ }^{235} \mathrm{U}$ makeup, the fuel types show that the ${ }^{232} \mathrm{U}$ concentrations begin to asymptotically approach an equilibrium concentration over the course of the $30 \mathrm{GWd} / \mathrm{Mg}_{\text {Iнм }}$ depletion. The cases of PWR Recycle fuel with ${ }^{233} \mathrm{U}$ and ${ }^{235} \mathrm{U}$ makeup have an initial ${ }^{232} \mathrm{U}$ concentration that remains near constant. The AHWR Equilibrium fuel type also has initial ${ }^{232} \mathrm{U}$ present in the fuel, but this increases over the course of the depletion.

As observed in Figure 37, the lowest ${ }^{232} \mathrm{U}$ concentrations at discharge occurs with the MSR. In this case, the ${ }^{232} \mathrm{U}$ concentration is likely artificially low due to the assumptions of the model, which removes all protactinium isotopes $\left({ }^{232} \mathrm{~Pa},{ }^{233} \mathrm{~Pa}\right.$ ) but only feeds in ${ }^{233} \mathrm{U}$ and thorium. While some degree of ${ }^{232} \mathrm{U}-{ }^{233} \mathrm{U}$ separation is possible using the different half-lives of ${ }^{232} \mathrm{~Pa}$ ( 1.32 days) and ${ }^{233} \mathrm{~Pa}$ ( 26.967 days), this separation is likely not $100 \%$. Therefore, a finite quantity of ${ }^{232} \mathrm{U}$ would be fed back into the fuel salt along with the ${ }^{233} \mathrm{U}$, instead of the pure ${ }^{233} \mathrm{U}$ that the model assumes. The PWR Hetero $90 \% \mathrm{U}$ also has low ${ }^{232} \mathrm{U}$ concentration at discharge, but this is due to the low thorium MOX fraction initially present in the fuel. The atom concentration of ${ }^{232} \mathrm{U}$ is given in terms of total $\mathrm{U}$, so $\mathrm{U}$-Th MOX has initial uranium (mostly ${ }^{238} \mathrm{U}$ ) that dilutes ${ }^{232} \mathrm{U}$ at discharge. Other initial PWR fuel types with greater thorium MOX fractions range up to a few hundred $\mathrm{ppm}$ of ${ }^{232} \mathrm{U}$. The PFBR thorium blankets have no initial uranium present, but they produce ${ }^{233} \mathrm{U}$ with relatively low concentrations of ${ }^{232} \mathrm{U}$ at $\sim 10-20 \mathrm{ppm}$. The AHWR fuels have the greatest concentrations of ${ }^{232} \mathrm{U}$, ranging from a few hundred ppm early in the cycle to near 1000 ppm at discharge. The AHWR fuels primarily use Pu-Th MOX without initial uranium present to dilute the ${ }^{232} \mathrm{U}$. 


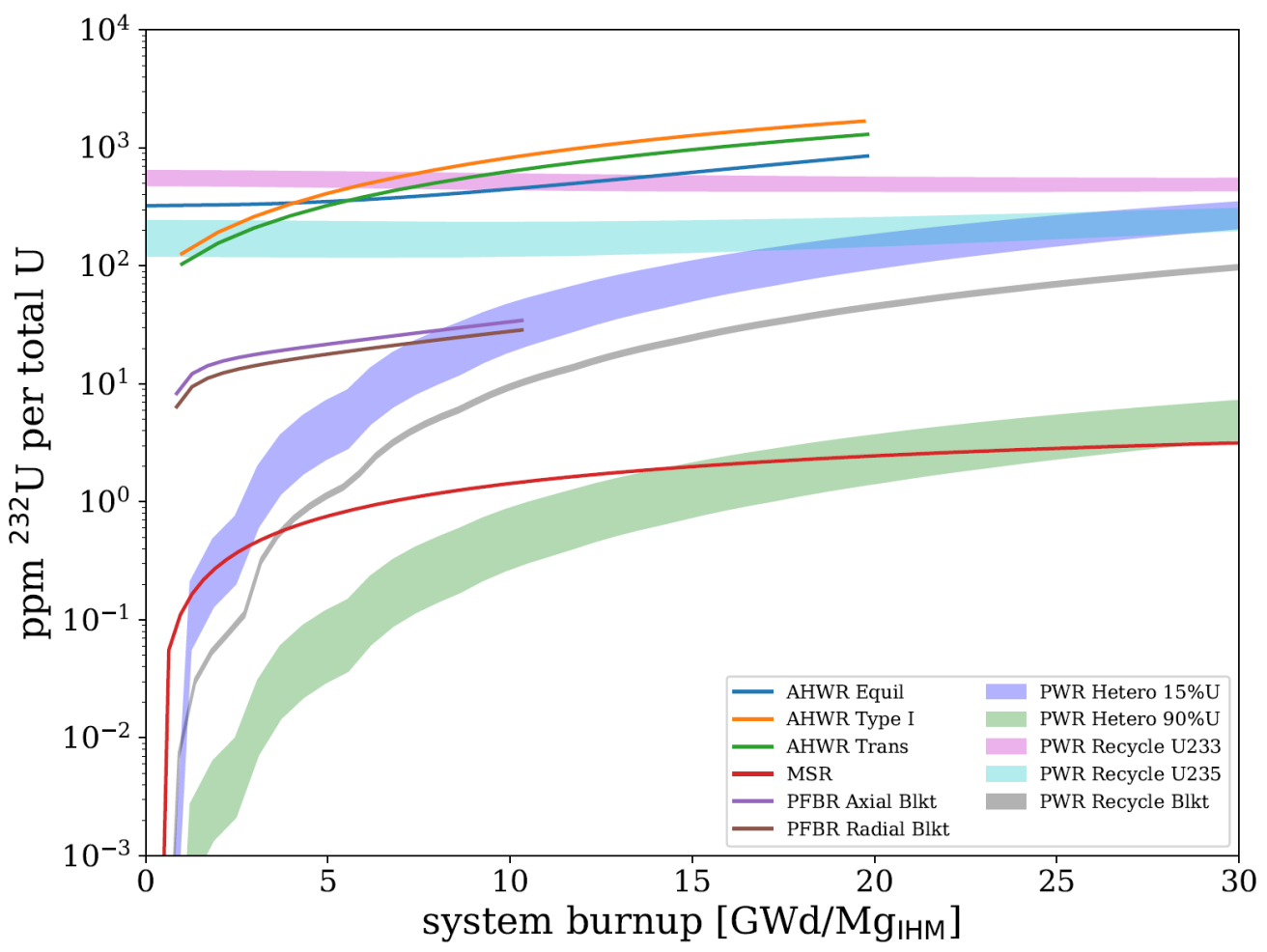

Figure 37. Comparison across reactor types of the mass concentration of ${ }^{233} \mathrm{U}$ per initial thorium.

The higher ${ }^{232} \mathrm{U}$ content in thorium fuel cycles than $\mathrm{U} / \mathrm{Pu}$ fuel cycles may drive the need for remote measurements due to the high gamma radiation dose associated with ${ }^{232} \mathrm{U}$ progeny. Therefore, safeguards technology requirements will need to be scoped accordingly. Furthermore, for safeguards gamma radiation measurements, ${ }^{232} \mathrm{U}$ progeny (predominantly ${ }^{208} \mathrm{Tl}$ ) will make it challenging to detect the low-intensity gamma rays from ${ }^{233} \mathrm{U}$ (associated with ${ }^{233} \mathrm{U}$ alpha decay to ${ }^{229} \mathrm{Th}$ ) because of the high Compton continuum. Analysis methods will thus need to be adapted to quantify ${ }^{232} \mathrm{U}$ and detect ${ }^{233} \mathrm{U}$ in the presence of ${ }^{232} \mathrm{U}$. The effect of ${ }^{232} \mathrm{U}$ on ${ }^{233} \mathrm{U}$ detection is discussed further in Section 3.

\subsection{Spent Nuclear Fuel Simulation Results}

Developed at ORNL, the SCALE software package includes modules to compute material compositions under irradiation and decay [9]. ORIGEN calculates time-dependent concentrations, activities, and radiation source terms, and it is used with neutron transport codes to produce burnup-dependent nuclide cross-section data that can be parameterized and combined into a format known as a reactor library. Parameterized reactor libraries are used by ORIGEN's Automated Rapid Processing module to quickly produce interpolated cross-sections that are used to compute nuclide compositions and gamma ray emission spectra under specified irradiation conditions and during decay intervals. This output is used as input to the GADRAS code [46] to simulate measured gamma ray spectra for a variety of instrument types and source geometries. This section discusses PWR assembly spent fuel dose rate and AHWR gamma spectra modeling and results. 


\subsubsection{PWR Spent Fuel Assembly Dose Rate}

It has been previously documented that fresh fuel assemblies using ${ }^{233} \mathrm{U}$ may require increased radiation protection measures for personnel in fabrication facilities due to gamma radiation from ${ }^{232} \mathrm{U}$ decay products [47]. The effect of thorium fuels on the dose rate of SNF, which would be under containment and surveillance, is less well known. To understand the possible impacts to safeguards measurement technologies, the bare (i.e., unshielded) gamma dose rate from a thorium-bearing PWR fuel assembly was compared to the dose rate from a typical $\mathrm{UO}_{2}$ PWR fuel assembly.

The radiation transport code MAVRIC (Monaco with Automated Variance Reduction using Importance Calculations), which is part of the SCALE suite, was employed to determine the multigroup gamma dose-rate response functions at $1 \mathrm{~m}$ from bare spent PWR fuel assemblies. For the cooling times on the order of decades, the dose rate of bare spent fuel assemblies was dominated by the contribution from gamma rays; therefore, the dose rates due to neutrons were not simulated. The 3D assembly model (see Figure 38) used the same Westinghouse $17 \times 17$ lattice geometry employed for the PWR nuclide inventory calculations. The $\mathrm{UO}_{2}$ assembly was composed of a homogeneous fuel material while the $\mathrm{UO}_{2}-\mathrm{ThO}_{2}$ assembly used a heterogeneous seed-and-blanket design, with the U-Th MOX fuel pins on the assembly periphery. The peripheral fuel pins were more exposed and partially shield the gamma rays from the interior pins. All other factors being equal, a peripheral fuel pin's contribution to the dose rate at $1 \mathrm{~m}$ will be greater than for an interior fuel pin. The 3D model includes fuel, cladding, and guide tubes but does not include structural material such as spacer grids, nozzles, or springs. These structural materials would provide an insignificant degree of shielding and backscattering. Neutron activation of structural materials was also not considered. The contribution to the gamma dose rate due to activation of structural materials is expected to be a few percent at most, and neutron activation due to $\mathrm{UO}_{2}-\mathrm{ThO}_{2}$ and $\mathrm{UO}_{2}$ fuel (all other factors being equal) is expected to have negligible differences.

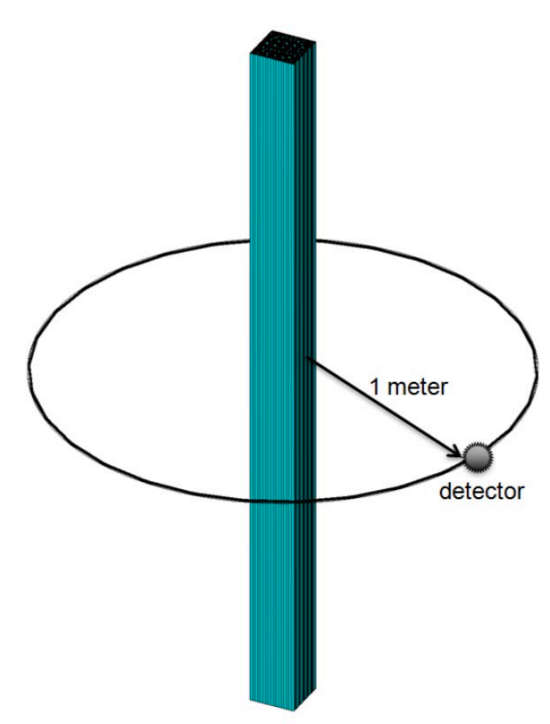

Figure 38. 3D PWR assembly with dose-rate detector at $1 \mathrm{~m}$ from the axial middle of the assembly.

Because many enrichments and MOX fractions are possible, the dose rate from an $\mathrm{UO}_{2}-\mathrm{ThO}_{2}$ assembly must be compared to an equivalent $\mathrm{UO}_{2}$ assembly to make a reasonable judgment about differences in dose rate. To determine equivalent $\mathrm{UO}_{2}$ and $\mathrm{UO}_{2}-\mathrm{ThO}_{2}$ lattices, lifetime-averaged reactivity was 
used as a metric. The lifetime-averaged reactivity of a lattice is the value of the neutron multiplication factor, $\mathrm{k}_{\text {eff, }}$ for an uncontrolled reflected lattice averaged over the lifetime of the depletion. Equivalent lifetime-averaged reactivities mean that the two fuel designs evolve with similar neutronics when averaged over their depletion lifetime. Interchanging lifetime-averaged reactivity equivalent lattices could be the first (but certainly not the last) step in optimizing a core that replaces some number of $\mathrm{UO}_{2}$ fuel lattices with a $\mathrm{UO}_{2}-\mathrm{ThO}_{2}$ fuel lattices. The equivalency between the two lattices is limited because the reflected lattice calculation does not consider the effect of differing adjacent lattices as found in a real core design. Additionally, real core designs are optimized taking into consideration many factors beyond reactivity alone, including power distribution, fuel temperature, neutron kinetics, and accident scenarios.

The $\mathrm{UO}_{2}-\mathrm{ThO}_{2}$ assembly considered for the dose-rate comparison used a heterogeneous seed-andblanket design with the seed composed of $\mathrm{UO}_{2}$ enriched to $4 \mathrm{wt} \%{ }^{235} \mathrm{U}$ and the blanket composed of $55 \% \mathrm{ThO}_{2}$ and $45 \% \mathrm{UO}_{2}$ enriched to $4 \mathrm{wt} \%{ }^{235} \mathrm{U}$. An assembly of this composition was considered because the enrichment is typical for a PWR assembly and because the blanket is in the middle range of MOX fractions under consideration. The equivalent $\mathrm{UO}_{2}$ assembly was composed of a homogeneous fuel material enriched to $2.53 \mathrm{wt} \%{ }^{235} \mathrm{U}$, which is on the lower end of the range typically employed in modern PWRs. As previously discussed, fuel employing $\mathrm{ThO}_{2}$ requires higher enrichment $\mathrm{UO}_{2}$ to compensate for the fissile ${ }^{235} \mathrm{U}$ displaced by the fertile ${ }^{232} \mathrm{Th}$.

The top plot in Figure 39 shows the gamma dose rate due to a $\mathrm{ThO}_{2}-\mathrm{UO}_{2} \mathrm{PWR}$ assembly after shutdown from the reactor (orange dotted line) as compared to an equivalent $\mathrm{UO}_{2}$ assembly after shutdown from the reactor (blue solid line). Because the gamma dose rate rapidly decreases after shutdown, falling by an order of magnitude after a few years, the top plot uses a logarithmic scale on the $y$-axis. The top plot demonstrates that the gross behavior of the bare gamma dose rate from a $\mathrm{ThO}_{2}-\mathrm{UO}_{2}$ PWR assembly is like an equivalent $\mathrm{UO}_{2}$ assembly of the same burnup.

The bottom plot shows the relative difference of the dose rates between the two assemblies. From this plot, small but significant differences up to $15 \%$ can be observed in the dose rate at various times after shutdown. After $\sim 1$ month, the dose rate from the $\mathrm{ThO}_{2}-\mathrm{UO}_{2} \mathrm{PWR}$ assembly is $\sim 15 \%$ greater than the equivalent $\mathrm{UO}_{2}$ assembly. During this time, it is likely the assembly would still reside in the reactor core itself, the transfer bay, or the spent fuel pool, depending on the refueling outage schedule. The relative difference in the dose rates begins to rapidly drop such that, after $\sim 6$ months, the dose rates from both assembly types are approximately the same. After $\sim 3$ years, the dose rate from the $\mathrm{ThO}_{2}-\mathrm{UO}_{2}$ PWR assembly is $\sim 11 \%$ lower than the equivalent $\mathrm{UO}_{2}$ assembly. This is a somewhat surprising finding because it shows that the fission product gamma ray emission rates dominate the gamma ray emission rates of the ${ }^{232} \mathrm{U}$ daughter radionuclides. The relative difference in dose rates begins to gradually approach zero again over the course of the next 11 years. Fourteen years after shutdown, again the dose rates from the assemblies are approximately equal. During this time, the fuel assembly is likely to be in a dry storage cask. Gradually over the next decades, the relative difference in dose rate will continue to rise such that, at 100 years after shutdown, the dose rate from the $\mathrm{ThO}_{2}-\mathrm{UO}_{2}$ assembly will be $\sim 8 \%$ greater than the equivalent $\mathrm{UO}_{2}$ assembly. 


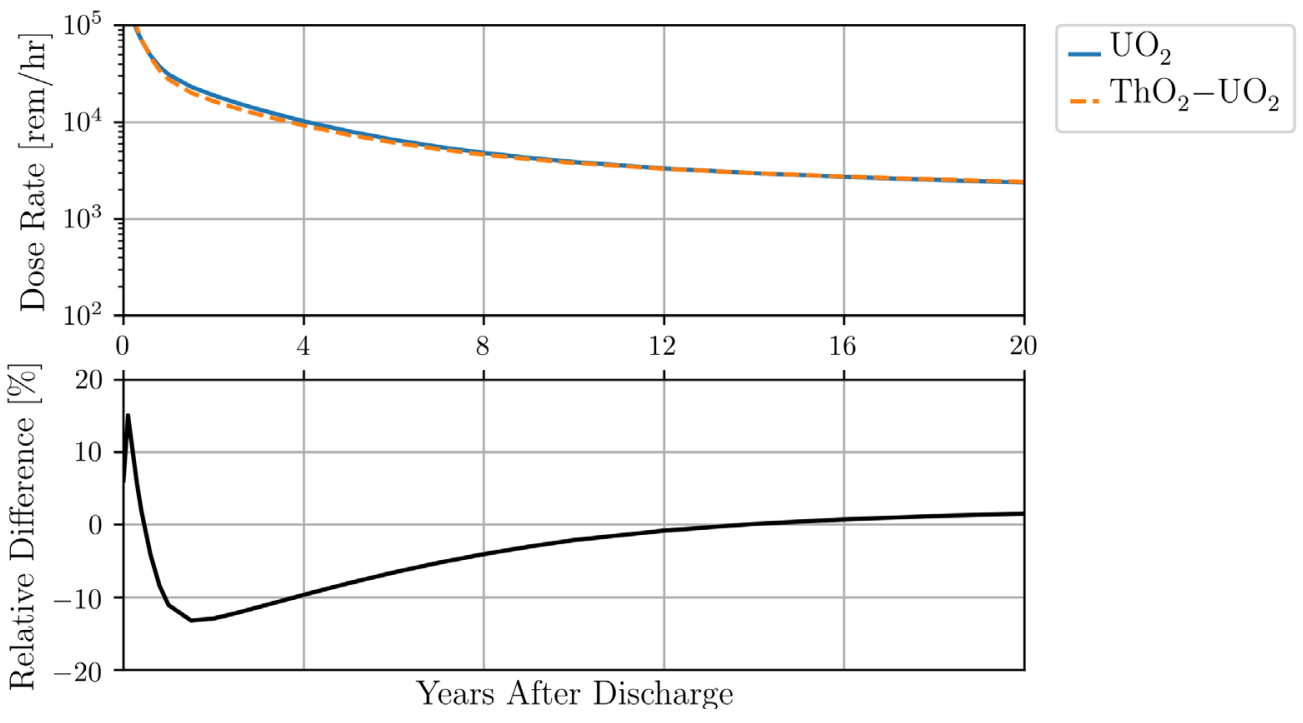

Figure 39. (Top) The absolute gamma dose rates from a bare $\mathrm{ThO}_{2}-\mathrm{UO}_{2} \mathrm{PWR}$ assembly and an equivalent $\mathbf{U O}_{2}$ assembly. (Bottom) The relative difference between the two dose rates, where a positive relative difference indicates a greater dose rate due to $\mathrm{a} \mathrm{ThO}_{2}-\mathrm{UO}_{2}$ assembly.

While fresh fuel fabrication facilities that use ${ }^{233} \mathrm{U}$ may require increased radiation protection measures due to the decay products from trace quantities of ${ }^{232} U$, the dose rate from SNF containing ${ }^{232} \mathrm{U}$ decay products is not very different from conventional spent $\mathrm{UO}_{2}$ fuel. This is because the contribution to the gamma dose rate due to ${ }^{232} \mathrm{U}$ decay products is small compared to the contribution from fission products. Fission product concentration is directly proportionate to burnup, and although there are differences in the fission product yield curves between ${ }^{233} \mathrm{U}$ and ${ }^{235} \mathrm{U}$, these differences do not greatly change the concentrations of the primary fission product contributors to gamma dose rate. Therefore, two bare assemblies at the same burnup will have similar dose rates regardless of the primary fissile isotopes used in the fuel. The dose rate of SNF is several orders of magnitude greater than that of the separated uranium, even with ${ }^{232} \mathrm{U}$ decay products. Although much lower than SNF, the dose rate from separated uranium with ${ }^{232} \mathrm{U}$ decay products is still significantly greater than the dose rate from the LEU typically used in fuel fabrication facilities, which is why increased radiation protection may be required for facilities using ${ }^{233} \mathrm{U}$.

For safeguards, the comparable dose rates mean that for thorium-bearing spent PWR fuel, the monitoring and surveillance techniques and instrumentation will likely not be limited by greater dose rate requirements than those used for conventional $\mathrm{UO}_{2}$ fuel. Therefore, from a radiation source term perspective alone, safeguards technology developments for conventional spent uraniumbased PWR fuel, including detector and electronics developments, would be expected to apply to the gamma ray emission rates anticipated from spent thorium-based PWR fuels.

\subsubsection{AHWR Gamma Spectra Modeling and Results}

Four types of AHWR assemblies were modeled using the TRITON and ORIGEN modules from the SCALE-6.2 code system [9]. A standard Westinghouse $17 \times 17$ PWR was also modeled to represent a conventional once-through ${ }^{235} \mathrm{U}$ fuel cycle as a baseline for comparison. Table 24 shows the initial elemental compositions of the five assembly types and the ${ }^{235} \mathrm{U}$ enrichment level (i.e., mass of ${ }^{235} \mathrm{U}$ divided by total mass of $\mathrm{U}$ ) and the ${ }^{240} \mathrm{Pu}$ vector (i.e., mass of ${ }^{240} \mathrm{Pu}$ divided by total mass of $\mathrm{Pu}$ ). 
Table 24. Starting compositions of the four types of AHWR assemblies and a regular $17 \times 17$ PWR assembly.

\begin{tabular}{|l|c|c|c|c|c|}
\hline & Initial Core I & Initial Core II & Transient Core Equilibrium Core & $\mathbf{1 7 \times 1 7}$ \\
\hline Thorium & $97.892 \%$ & $0.000 \%$ & $94.732 \%$ & $95.801 \%$ & $0.000 \%$ \\
\hline Uranium & $0.000 \%$ & $98.499 \%$ & $0.000 \%$ & $1.556 \%$ & $100.000 \%$ \\
\hline Plutonium & $2.108 \%$ & $1.501 \%$ & $5.268 \%$ & $2.643 \%$ & $0.000 \%$ \\
\hline${ }^{235}$ U enrichment & $0.000 \%$ & $0.261 \%$ & $0.000 \%$ & $0.101 \%$ & $4.000 \%$ \\
\hline${ }^{240}$ Pu percentage & $24.586 \%$ & $24.586 \%$ & $24.586 \%$ & $24.586 \%$ & $0.000 \%$ \\
\hline
\end{tabular}

For each of these five assemblies, simulated irradiation of $40 \mathrm{GWd} / \mathrm{MTU}$ followed by cooling times ranging from 30 days to 30 years was modeled. For each decay time, the simulations produced a list of isotopic concentrations, which were then used with gamma yield libraries to produce inputs for the GADRAS code [46]. Because full particle transport simulations with a code like Monte Carlo N-Particle Transport (MCNP) were beyond the scope of this task, the spectra were generated using the assemblyaveraged materials as a point source. Specifically, for the GADRAS simulations, the gamma emissions were treated as a point source $100 \mathrm{~cm}$ from a high-purity germanium (HPGe) detector. In a sense, this most closely represents a DA measurement where sample self-attenuation is relatively minor. The general features of the spectra are similar, particularly for the purpose of peak identification. The main difference is that features like cladding can completely attenuate low-energy gamma rays below $\sim 100 \mathrm{keV}$.

To best visualize differences between samples, the source intensity of each assembly was normalized to create a count rate of 10,000 counts per second in the detector response. Each spectrum has a live time of 1000 seconds for a total of 10 million counts. This normalization allows for the best visual comparison of the shapes and peaks of the individual spectra. In the following sections, spectra for cooling times of 30 days, 1 year, 5 years, and 20 years are presented and compared. The features of spectra with 10- and 30-year cooling times were similar to their close neighbors and omitted for the sake of brevity.

Several fundamental assumptions are made when treating the assembly-averaged materials as a point source. Some isotopes (e.g., americium-241 $\left[{ }^{241} \mathrm{Am}\right]$ ) would never be seen in measuring an assembly because their low-energy gamma rays would be stopped by the cladding on the fuel rods. Furthermore, real SNF assemblies have radial zoning with different materials on each of the three rings. On a spectrum of the entire assembly, the outer fuel pins would be responsible for most of the gamma signatures.

\subsubsection{30-day Spectra}

Figure 40 shows the gamma spectra of the modeled assembly types at 30 days of cooling time. The most obvious difference at this scale is at high energies, especially the continuum difference below the $1596 \mathrm{keV}$ lanthanum-140 $\left({ }^{140} \mathrm{La}\right)$ peak. This difference is largely attributable to the fact that the three thorium MOX assemblies (i.e., Initial Core I, Transient Core, Equilibrium Core) have a significant number of gamma and $\mathrm{x}$-rays from ${ }^{233} \mathrm{~Pa}$. Because of the normalization, these relatively low-energy peaks tend to depress the higher end of those spectra. Also notable in Figure 40 is a near lack of the $2615 \mathrm{keV}$ peak representative of ${ }^{232} \mathrm{U}$. This is because the ${ }^{232} \mathrm{U}$ daughters have not come into secular equilibrium at this point in time and the ${ }^{208} \mathrm{Tl}$ isotope that produces this high-energy gamma ray has only reached $3 \%$ of its maximum buildup. 
Figure 41 shows a close-up view of the $0-500 \mathrm{keV}$ energy region for the simulated spectra at 30 days of cooling time. Here, several peaks belonging to ${ }^{233} \mathrm{~Pa}$ are clearly visible in the thorium MOX spectra (i.e., Initial Core I, Transient Core, Equilibrium Core), but not in the U-Pu MOX (Initial Core I) or $\mathrm{UO}_{2}$ $(17 \times 17)$ spectra. This ${ }^{233} \mathrm{~Pa}$ comes from the absorption of a neutron by ${ }^{232} \mathrm{Th}$ and subsequent beta decay. Because the ${ }^{233} \mathrm{~Pa}$ has a 27 -day half-life, these peaks should be detectable only out to 2-300 days of cooling time. Other work considered ${ }^{233} \mathrm{~Pa}$ production rates in leading thorium fuel cycles as well as concepts and approaches for ${ }^{233} \mathrm{~Pa}$ monitoring $[5,48]$.

live-time $(s)=1000.00$

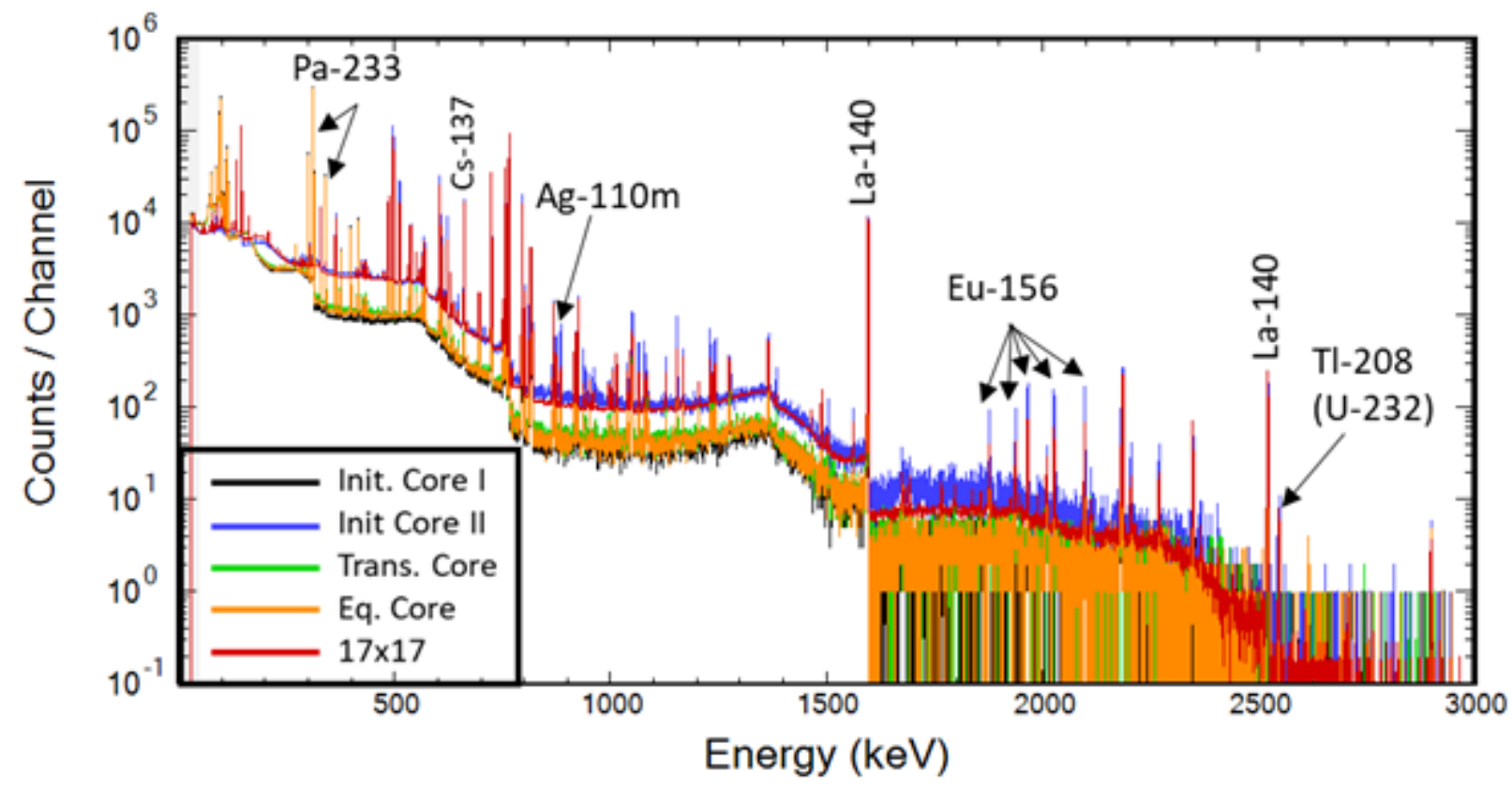

Figure 40. Simulated spectra of the five assembly types at 30 days after discharge from the reactor.

Figure 42 shows the spectra for the two nonthorium assemblies ( $17 \times 17$ and Initial Core II). Here, some differences can be noted between the peak heights of these two assemblies. Figure 43 shows a closeup view of the 500-1400 keV region, where these differences can be observed more clearly. Nearly all these peaks are gamma rays emitted by silver- $110 \mathrm{~m}\left({ }^{110 \mathrm{~m}} \mathrm{Ag}\right)$ or europium- $156\left({ }^{156} \mathrm{Eu}\right)$. These two isotopes are produced more often by the fission of ${ }^{239} \mathrm{Pu}$ than from lighter isotopes such $\operatorname{as}^{233} \mathrm{U}$ or ${ }^{235} \mathrm{U}$, and thus could be used for identification. ${ }^{110 \mathrm{~m}} \mathrm{Ag}$ production is also sensitive to the neutron energy spectrum, with harder spectra producing more of this isotope.

Figures 44 and 45 show the 30-day spectra for the three Th-MOX assemblies (Initial Core I, Transient Core, and Equilibrium Core). Here again, some of the peak intensities can be seen to vary, although the differences are not as large as those observed in Figures 42 and 43 . Most peaks that differ are produced by either ruthenium-106 $\left({ }^{106} \mathrm{Ru}\right)$ or ${ }^{110 \mathrm{~m}} \mathrm{Ag}$. 


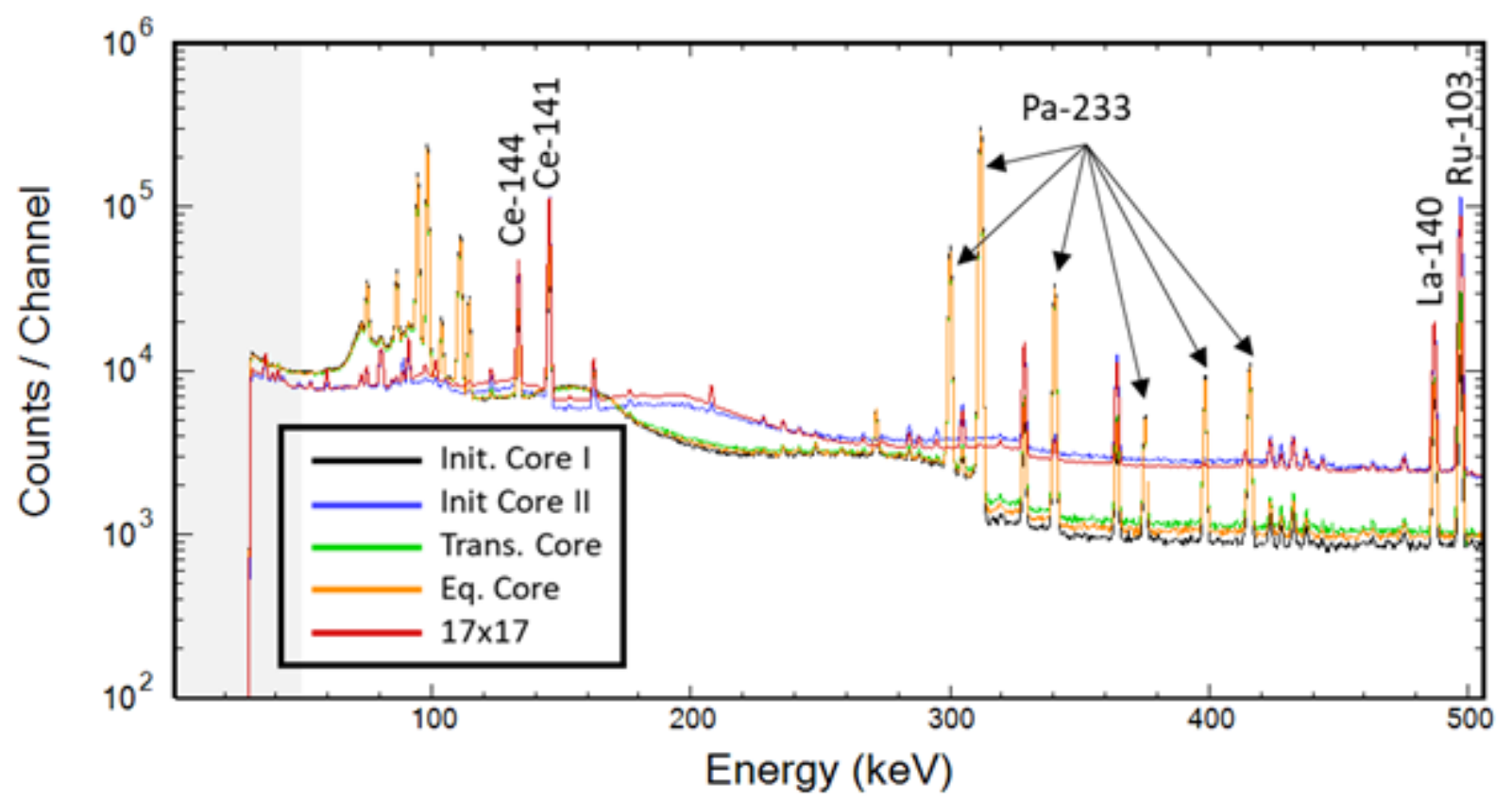

Figure 41. Close-up of the simulated spectra at 30 days of cooling time showing the $\mathbf{0 - 5 0 0} \mathrm{keV}$ energy range.

live-time $(s)=1000.00$

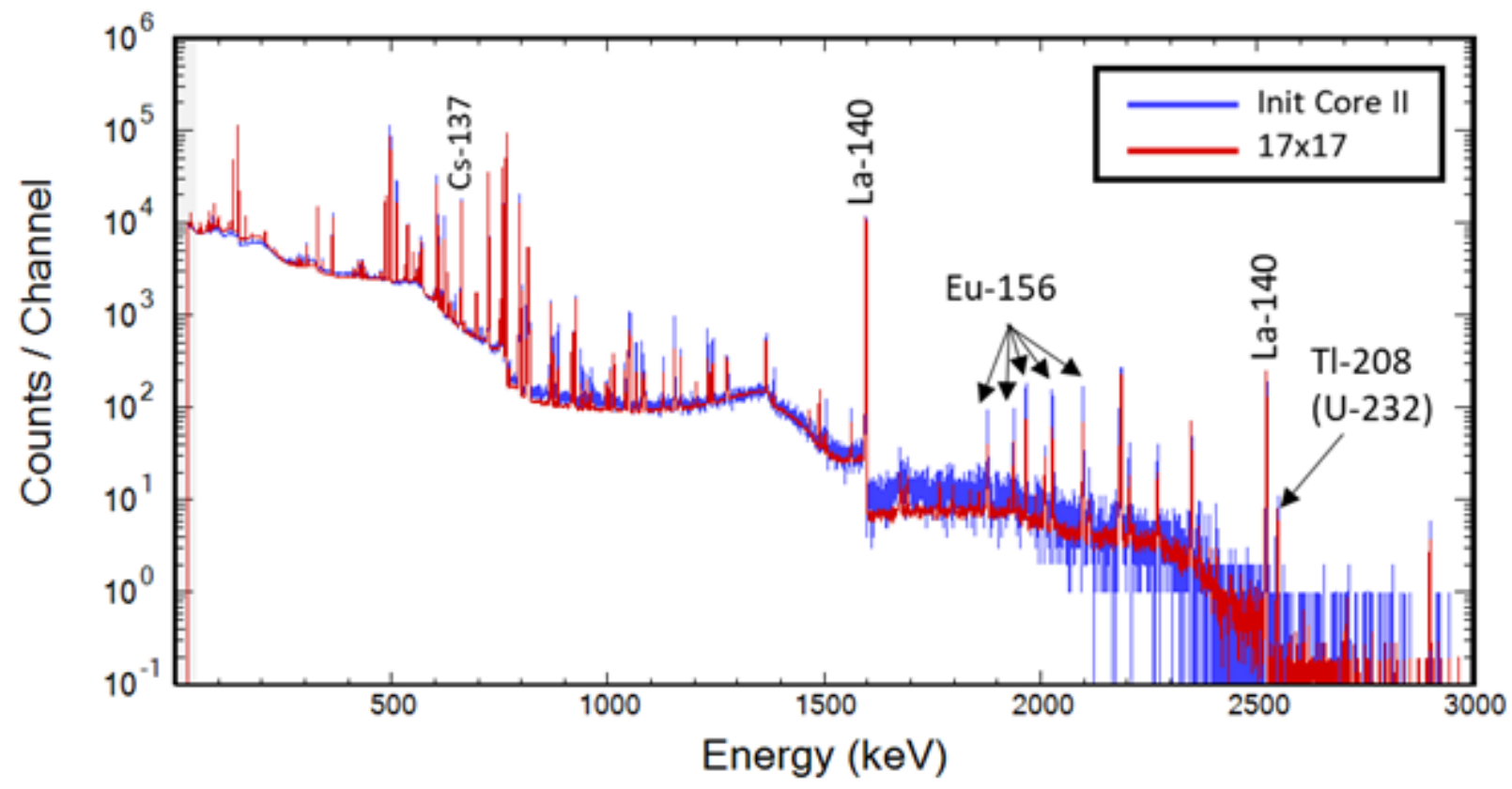

Figure 42. The simulated spectra of the two nonthorium assemblies at 30 days of cooling time. 


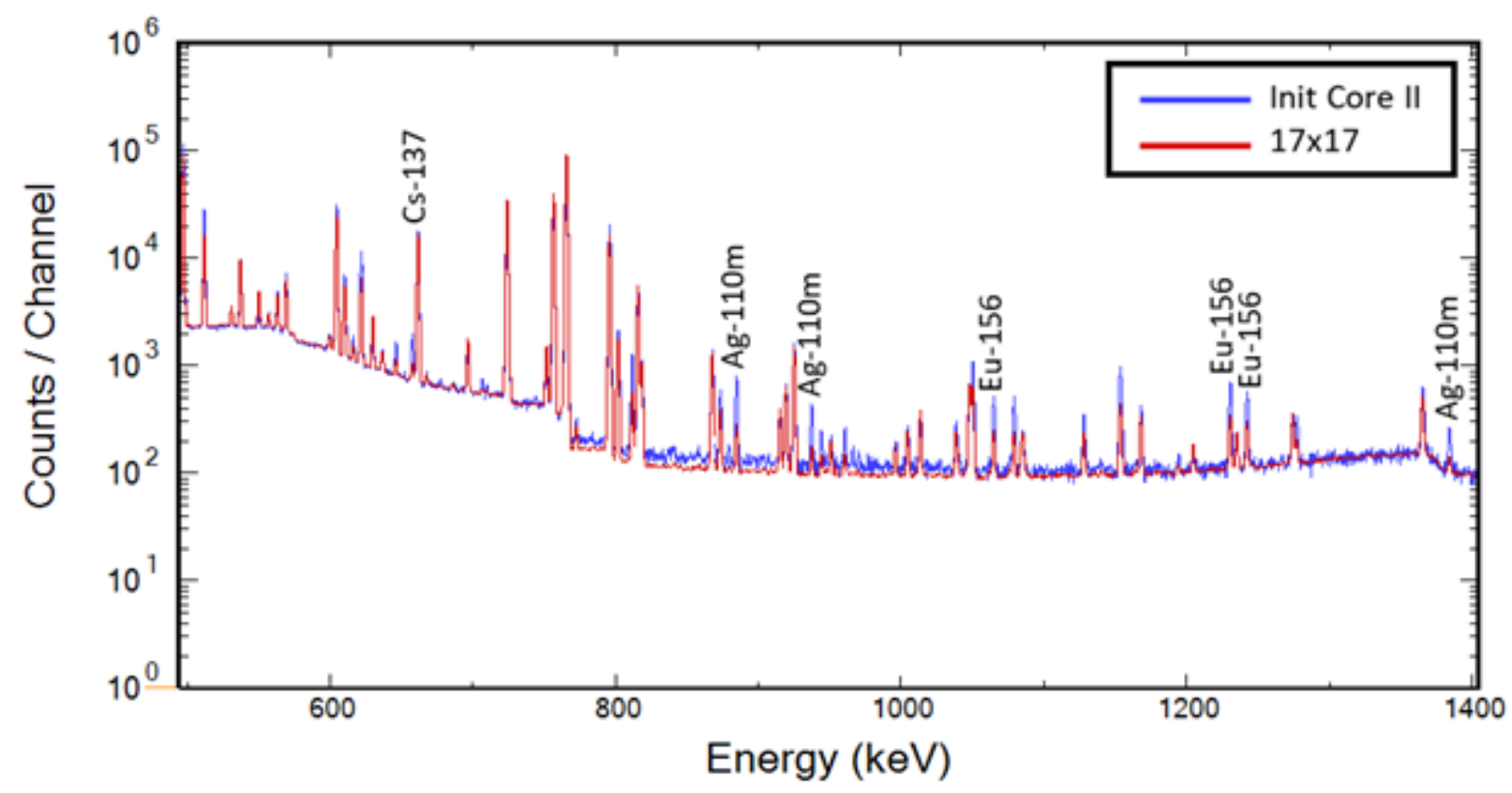

Figure 43. The $500-1400 \mathrm{keV}$ region of the simulated spectra for the two nonthorium assemblies at 30 days of cooling time.

Table 25 shows the concentrations of all the isotopes that were identified from the simulated 30-day gamma spectra using a spectral analysis tool. These isotopic concentrations were taken directly from the ORIGEN concentration files to avoid introducing errors from the peak-fitting process. Some of the isotopes with the largest differences between assembly types are highlighted in blue: ${ }^{233} \mathrm{~Pa}$, ${ }^{232} \mathrm{U}$, and, for the Initial Core II assembly, the ${ }^{110 \mathrm{~m}} \mathrm{Ag}$ and ${ }^{156} \mathrm{Eu}$ values. Except for the ${ }^{232} \mathrm{U}$, all these isotopes were clearly visible in the spectra presented in the previous figures. Also of note is that cesium-137 $\left({ }^{137} \mathrm{Cs}\right)$ remains almost constant between assembly types. This isotope is produced almost linearly with burnup, and due to its 30 year half-life will remain detectable in most spent fuel spectra for centuries. For NDA measurements, the ${ }^{137} \mathrm{Cs}$ intensity is often used as the denominator to form ratios with other isotopes. These ratios are much easier to measure than the absolute concentrations of isotopes, and they are useful for verifying the assemblies' irradiation history. Typically, with a gamma spectrum, it is not possible to measure the absolute concentration of an isotope because the geometry is complex. Therefore, calculating the ratio to ${ }^{137} \mathrm{Cs}$ provides a normalization factor. ${ }^{137} \mathrm{Cs}$ scales linearly with burnup, so it provides a means for comparison of two assemblies with the same burnup. Therefore, because of the presence of ${ }^{137} \mathrm{Cs}$ in all assembly types, the same methodology could apply to thorium fuels. 


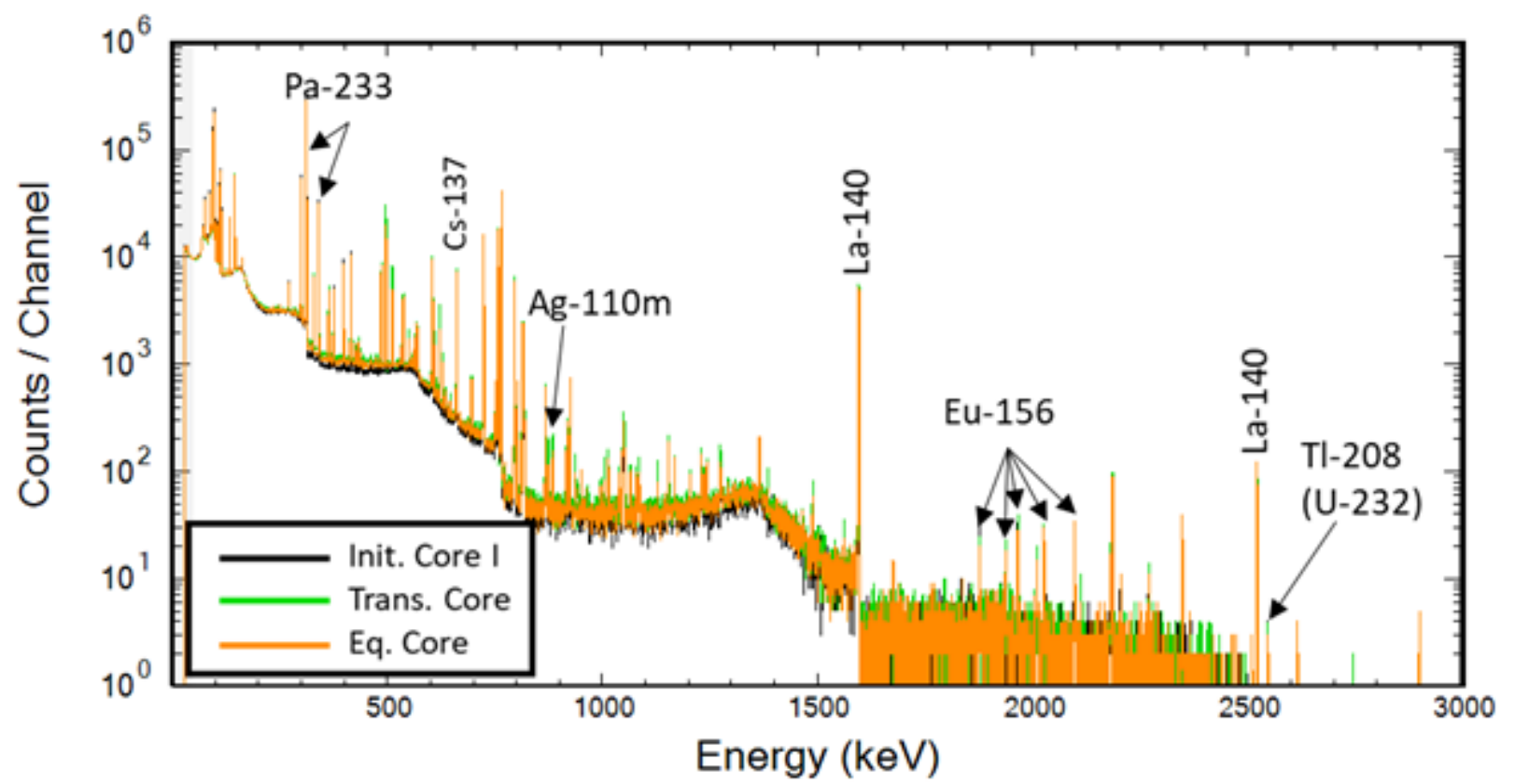

Figure 44. Simulated spectra of the three thorium-MOX assemblies at 30 days of cooling time.

live-time $(s)=1000.00$

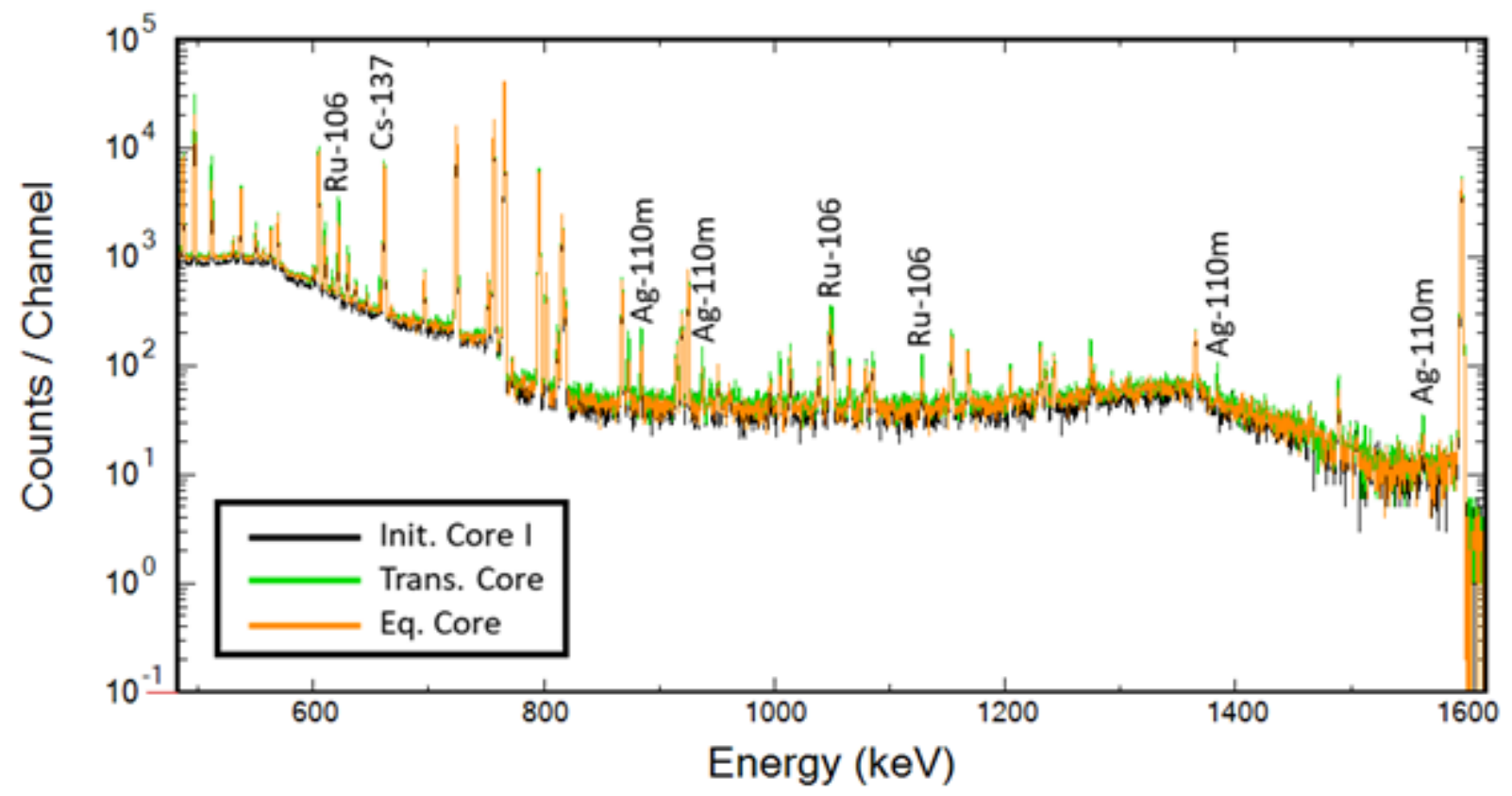

Figure 45. The 500-1600 keV region of the simulated spectra of the three thorium-MOX assemblies at 30 days of cooling time. 
Table 25. Isotopic concentrations (ppm) of the isotopes that could be identified from the simulated 30-day cooling time gamma spectra. Isotopic concentrations were taken directly from ORIGEN concentration files.

Blue highlighting shows isotopes where significant differences between spectra were observed.

\begin{tabular}{|l|c|c|c|c|c|}
\multicolumn{7}{|c}{ Initial Core I Initial Core II } & \multicolumn{4}{c|}{ Transient Core Equivalent Core } & $\mathbf{1 7 \times 1 7}$ \\
\hline${ }^{110 \mathrm{~m}} \mathrm{Ag}$ & 1.571 & 3.595 & 2.217 & 1.248 & 0.904 \\
\hline${ }^{241} \mathrm{Am}$ & 44.038 & 52.179 & 457.165 & 203.240 & 88.311 \\
\hline${ }^{140} \mathrm{Ba}$ & 4.073 & 3.384 & 3.803 & 3.973 & 3.597 \\
\hline${ }^{141} \mathrm{Ce}$ & 27.952 & 21.865 & 25.168 & 26.836 & 23.477 \\
\hline${ }^{144} \mathrm{Ce}$ & 315.588 & 257.667 & 288.272 & 307.258 & 306.061 \\
\hline${ }^{134} \mathrm{Cs}$ & 204.556 & 231.151 & 175.652 & 178.615 & 203.455 \\
\hline${ }^{136} \mathrm{Cs}$ & 0.163 & 0.159 & 0.180 & 0.178 & 0.174 \\
\hline${ }^{137} \mathrm{Cs}$ & 2458.261 & 2392.478 & 2431.424 & 2499.580 & 2363.606 \\
\hline${ }^{154} \mathrm{Eu}$ & 31.906 & 41.923 & 48.737 & 34.900 & 45.617 \\
\hline${ }^{155} \mathrm{Eu}$ & 12.517 & 18.412 & 17.344 & 12.830 & 13.822 \\
\hline${ }^{156} \mathrm{Eu}$ & 1.045 & 2.158 & 0.962 & 0.814 & 0.906 \\
\hline${ }^{131} \mathrm{I}$ & 0.582 & 0.560 & 0.562 & 0.573 & 0.538 \\
\hline${ }^{85} \mathrm{Kr}$ & 125.953 & 41.734 & 78.765 & 128.039 & 72.192 \\
\hline${ }^{140} \mathrm{La}$ & 0.618 & 0.513 & 0.577 & 0.603 & 0.545 \\
\hline${ }^{95} \mathrm{Nb}$ & 50.887 & 36.829 & 43.686 & 48.087 & 43.802 \\
\hline${ }^{147} \mathrm{Nd}$ & 0.768 & 0.861 & 0.818 & 0.794 & 0.902 \\
\hline${ }^{233} \mathrm{~Pa}$ & 330.515 & 0.000 & 222.857 & 260.469 & 0.000 \\
\hline${ }^{148 \mathrm{~m}} \mathrm{Pm}$ & 0.601 & 0.548 & 0.937 & 0.767 & 1.004 \\
\hline${ }^{106} \mathrm{Ru}$ & 107.815 & 397.229 & 272.386 & 161.012 & 231.496 \\
\hline${ }^{103} \mathrm{Ru}$ & 12.404 & 39.478 & 24.360 & 17.449 & 32.465 \\
\hline${ }^{124} \mathrm{Sb}$ & 0.047 & 0.045 & 0.036 & 0.044 & 0.038 \\
\hline${ }^{125} \mathrm{Sb}$ & 20.320 & 18.448 & 19.104 & 20.857 & 14.314 \\
\hline${ }^{232} \mathrm{U}$ & 46.978 & 0.000 & 33.735 & 35.272 & 0.001 \\
\hline${ }^{95} \mathrm{Zr}$ & 64.676 & 25.051 & 45.782 & 56.933 & 38.713 \\
\hline
\end{tabular}

\subsubsection{One-year Spectra}

Figure 46 shows the 1-year cooling time spectra for the five assembly types. The most obvious feature in these spectra is the $2615 \mathrm{keV}$ peak from the ${ }^{232} \mathrm{U}$ daughter product, thallium-208 $\left({ }^{208} \mathrm{Tl}\right)$. The two spectra lacking this peak are the Initial Core II (U-Pu MOX) and the $17 \times 17\left(\mathrm{UO}_{2}\right)$ assemblies, which contain no thorium in their initial composition. The spectra for these two assemblies are shown in Figure 47 . While these two spectra are quite similar, there are some visible differences. Figure 48 shows a closer view of the $800-1600 \mathrm{keV}$ region of these spectra where 5-6 peak areas appear to be significantly higher in the Initial Core II spectrum. These peaks result from the greater concentration of ${ }^{110 \mathrm{~m}} \mathrm{Ag}$ in the Initial Core II spectrum due to the plutonium in the fuel. 
Figure 49 shows the simulated spectra of the three thorium-MOX assemblies at 1 year of cooling time. Note that all these assemblies have a strong peak at $2615 \mathrm{keV}$, indicating the presence of ${ }^{232} \mathrm{U}$ daughter products. Figure 50 shows a close-up of the thorium-MOX assemblies. While there are some differences in peak intensities, they are less obvious than the differences in peak intensities between the two nonthorium assemblies. These differences in peak intensities are primarily due to differing concentrations of ${ }^{106} \mathrm{Ru}$ and ${ }^{110 \mathrm{~m}} \mathrm{Ag}$.

live-time $(s)=10000$

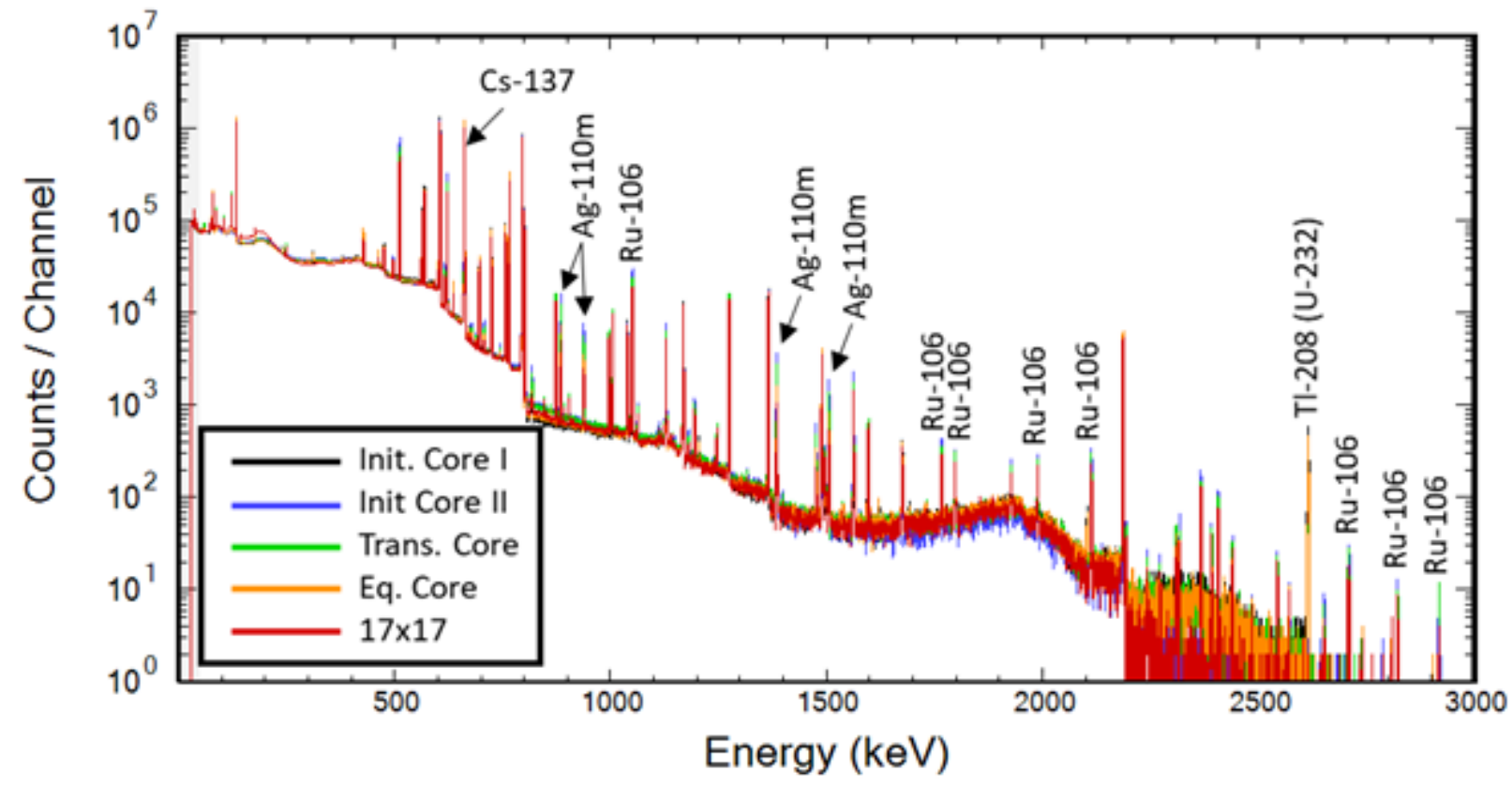

Figure 46. Simulated spectra of the five assembly types at 1 year after discharge from the reactor.

Table 26 shows the simulated isotopic concentrations in each assembly after 1 year of cooling time. The values of the three isotopes that produced clear visible differences in peak heights are highlighted. Also of note is the large difference in ${ }^{241} \mathrm{Am}$ concentrations. Unfortunately, this isotope is difficult to measure using NDA because its single strong peak at $60 \mathrm{keV}$ is easily attenuated by the fuel cladding.

\subsubsection{Five-year Spectra}

Figure 51 shows the simulated spectra of the five assembly types after 5 years of cooling time. At this point, the ${ }^{232} \mathrm{U}$ daughters are beginning to approach secular equilibrium, and the $2615 \mathrm{keV}{ }^{208} \mathrm{Tl}$ peak dominates the three thorium-MOX spectra. Figures 52 and 53 show the simulated spectra of the nonthorium spectra. The main observable differences are due to ${ }^{106} \mathrm{Ru}$ and ${ }^{110 \mathrm{~m}} \mathrm{Ag}$ peaks. After 5 years of cooling, these two isotopes become increasingly difficult to detect and quantify because of their relatively short half-lives-one year for ${ }^{106} \mathrm{Ru}$ and 250 days for ${ }^{110 \mathrm{~m}} \mathrm{Ag}$. 


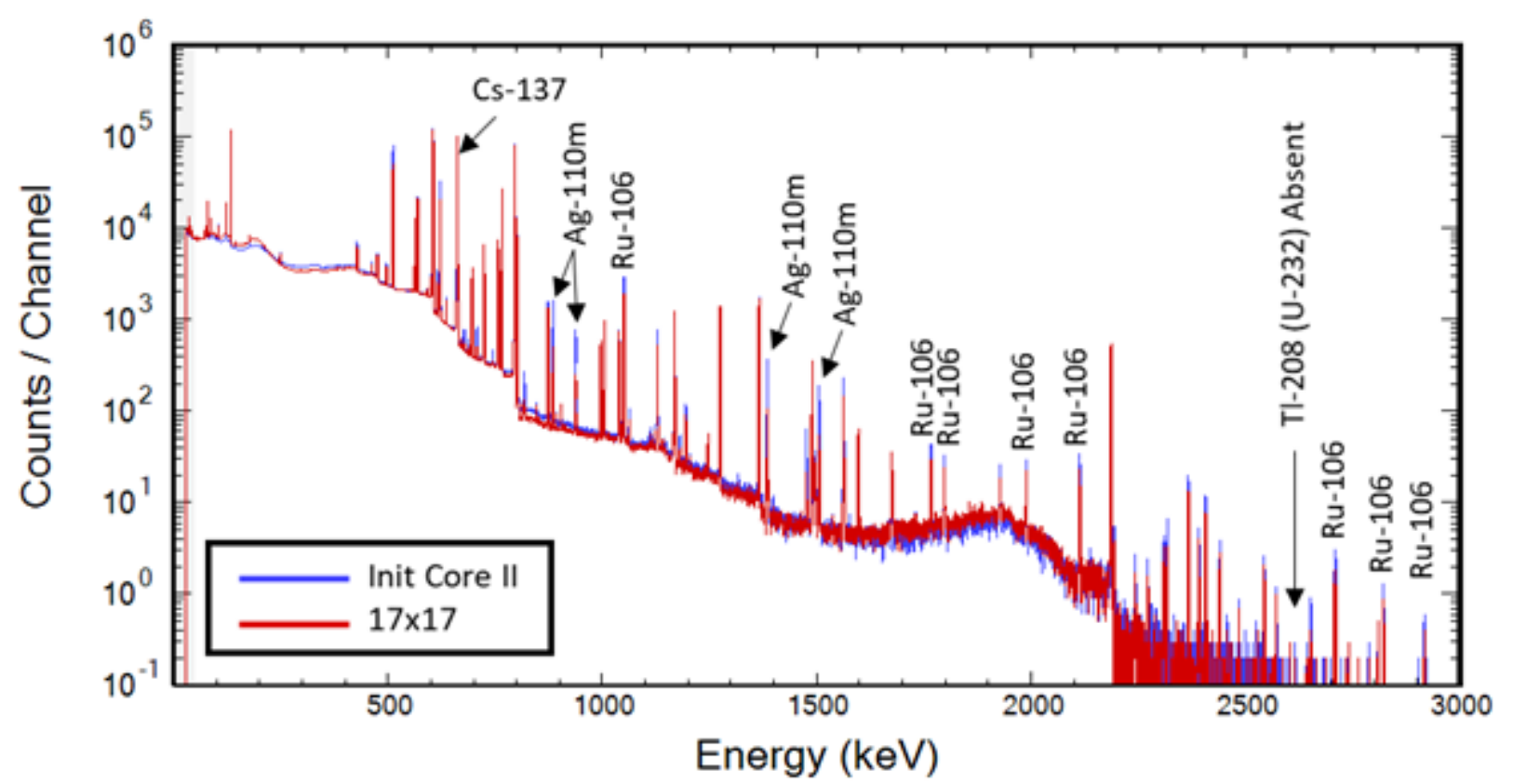

Figure 47. The simulated spectra of the two nonthorium assemblies at 1 year of cooling time.

live-time $(s)=1000.00$

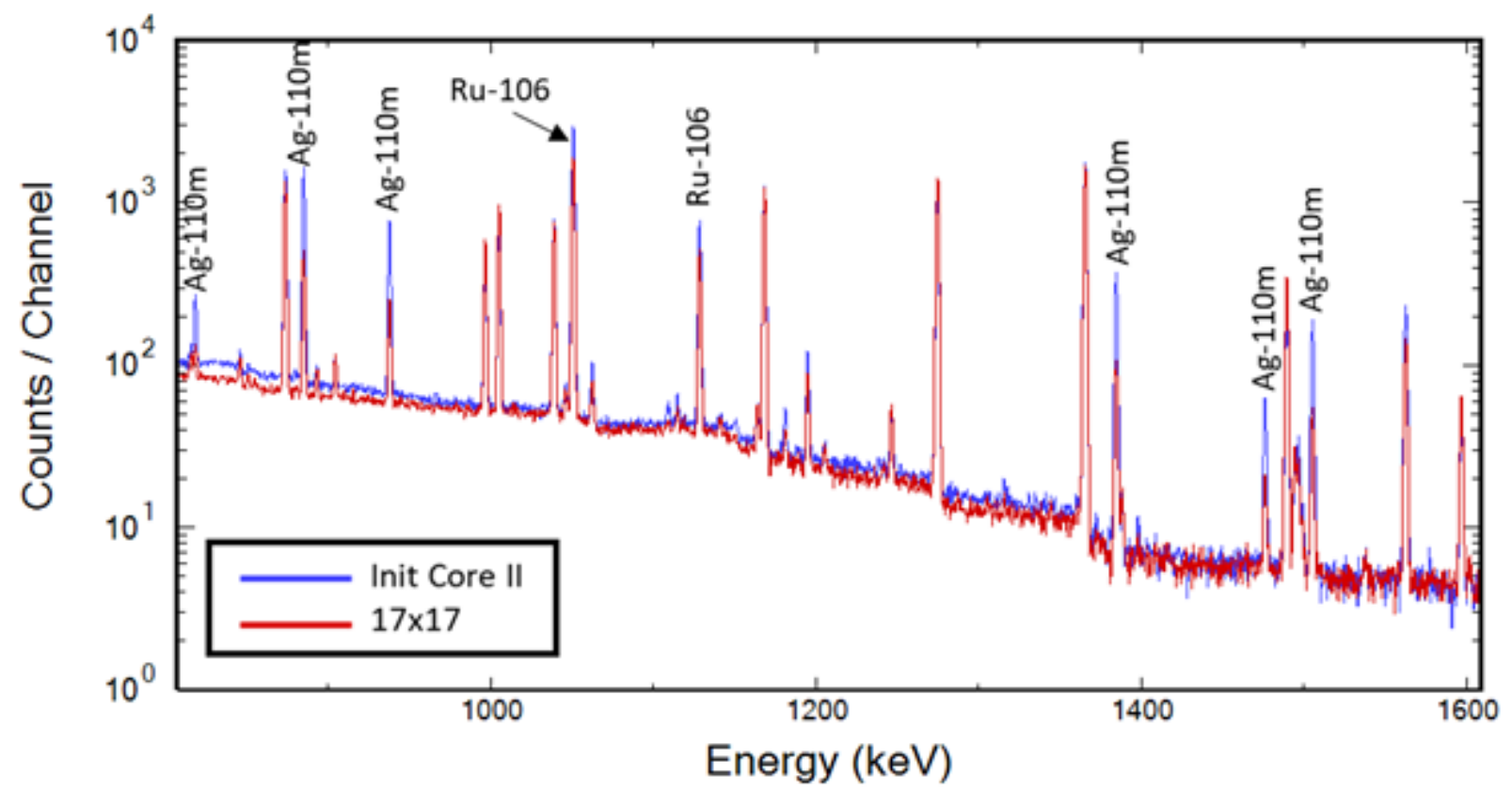

Figure 48. The $800-1600 \mathrm{keV}$ region of the simulated spectra for the two nonthorium assemblies at 1 year of cooling time. 


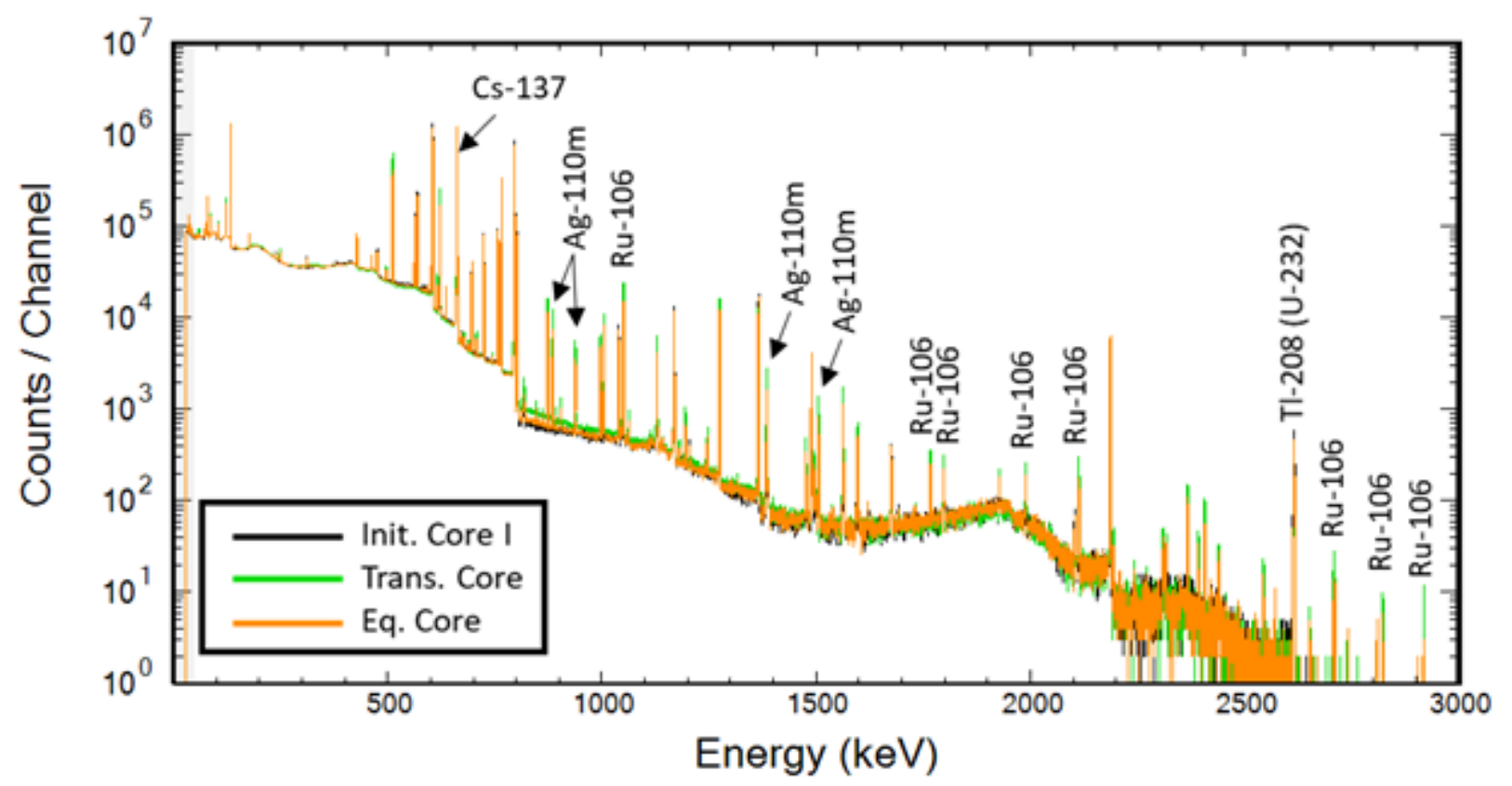

Figure 49. Simulated spectra of the three thorium-MOX assemblies at 1 year of cooling time.

live-time $(s)=10000$

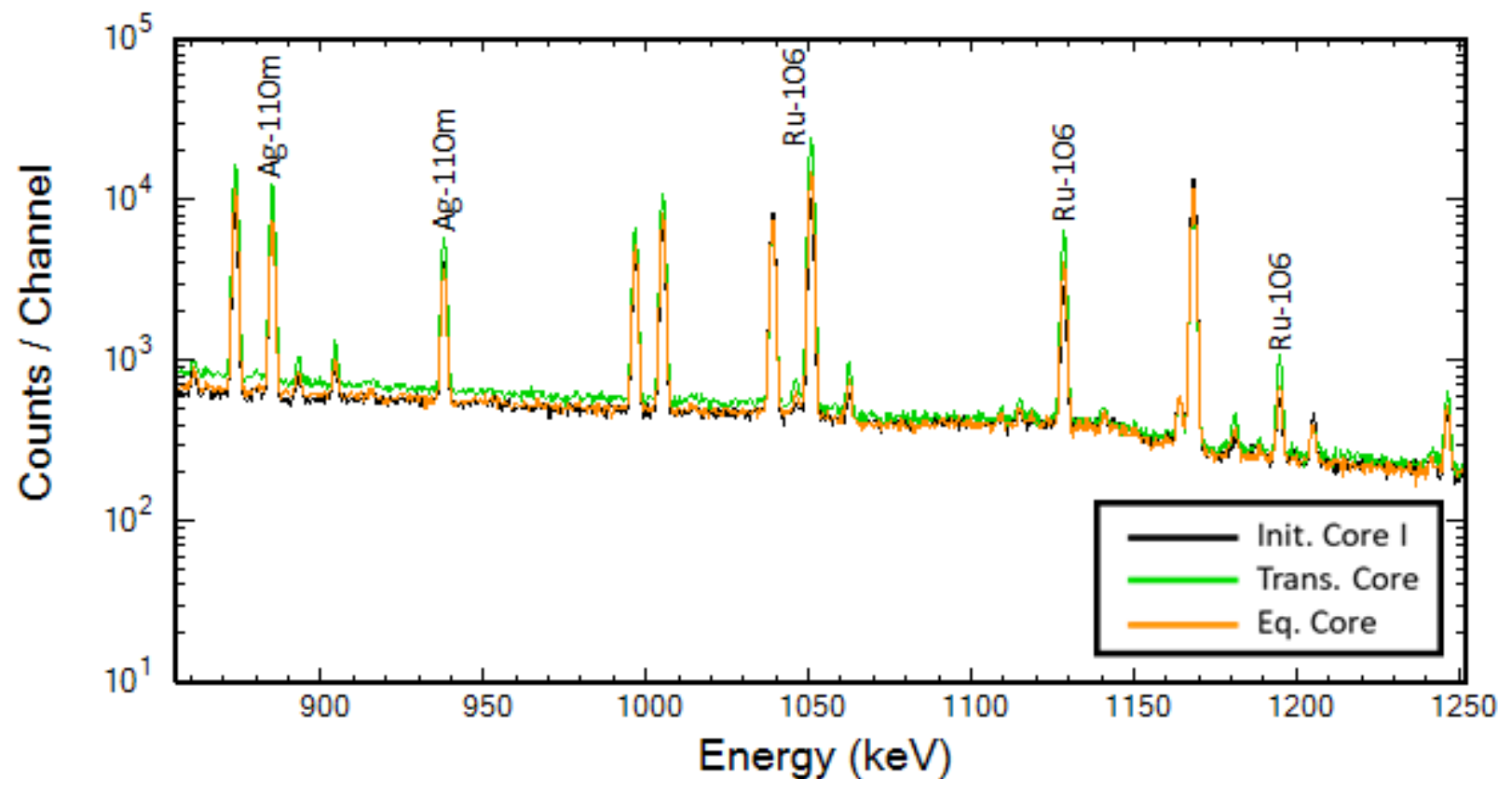

Figure 50. The $800-1250 \mathrm{keV}$ region of the simulated spectra of the three thorium-MOX assemblies at 1 year of cooling time. 
Table 26. Isotopic concentrations (ppm) of the isotopes that could be identified from the simulated 1-year cooling time gamma spectra. Isotopic concentrations were taken directly from ORIGEN concentration files.

\begin{tabular}{|c|c|c|c|c|c|}
\hline & Initial Core I & Initial Core II & Transition Core & Equilibrium Core & $17 \times 17$ \\
\hline${ }^{110 \mathrm{~m}} \mathrm{Ag}$ & 0.619 & 1.418 & 0.874 & 0.492 & 0.356 \\
\hline${ }^{241} \mathrm{Am}$ & 64.686 & 100.677 & 609.671 & 280.332 & 149.742 \\
\hline${ }^{141} \mathrm{Ce}$ & 0.022 & 0.017 & 0.020 & 0.021 & 0.018 \\
\hline${ }^{144} \mathrm{Ce}$ & 139.603 & 113.981 & 127.518 & 135.918 & 135.388 \\
\hline${ }^{134} \mathrm{Cs}$ & 150.319 & 169.863 & 129.077 & 131.256 & 149.509 \\
\hline${ }^{137} \mathrm{Cs}$ & 2406.789 & 2342.379 & 2380.478 & 2447.232 & 2314.098 \\
\hline${ }^{154} \mathrm{Eu}$ & 29.631 & 38.933 & 45.261 & 32.411 & 42.363 \\
\hline${ }^{155} \mathrm{Eu}$ & 10.949 & 16.105 & 15.171 & 11.223 & 12.090 \\
\hline${ }^{85} \mathrm{Kr}$ & 118.718 & 39.336 & 74.239 & 120.683 & 68.044 \\
\hline${ }^{95} \mathrm{Nb}$ & 2.275 & 1.644 & 1.954 & 2.149 & 1.951 \\
\hline${ }^{233} \mathrm{~Pa}$ & 0.060 & 0.000 & 0.040 & 0.047 & 0.000 \\
\hline${ }^{148 m} \mathrm{Pm}$ & 0.002 & 0.002 & 0.003 & 0.003 & 0.004 \\
\hline${ }^{144} \mathrm{Pr}$ & 0.006 & 0.005 & 0.005 & 0.006 & 0.006 \\
\hline${ }^{106} \mathrm{Ru}$ & 57.706 & 212.610 & 145.788 & 86.179 & 123.904 \\
\hline${ }^{103} \mathrm{Ru}$ & 0.033 & 0.106 & 0.065 & 0.047 & 0.087 \\
\hline${ }^{124} \mathrm{Sb}$ & 0.001 & 0.001 & 0.001 & 0.001 & 0.001 \\
\hline${ }^{125} \mathrm{Sb}$ & 16.146 & 14.658 & 15.179 & 16.572 & 11.374 \\
\hline${ }^{232} \mathrm{U}$ & 46.546 & 0.000 & 33.424 & 34.947 & 0.001 \\
\hline${ }^{91} \mathrm{Y}$ & 1.219 & 0.472 & 0.863 & 1.073 & 0.729 \\
\hline${ }^{95} \mathrm{Zr}$ & 1.929 & 1.394 & 1.657 & 1.822 & 1.653 \\
\hline
\end{tabular}

Figures 54 and 55 show the simulated 5-year spectra for the three thorium-MOX assemblies (Initial Core I, Transient Core, and Equilibrium Core). There are some easily observable differences in peak intensities, primarily due to differences in ${ }^{106} \mathrm{Ru}$ and ${ }^{110 \mathrm{~m}} \mathrm{Ag}$ concentrations. While smaller, there also are observable differences in the ubiquitous ${ }^{154}$ Eu peaks.

Table 27 shows the isotopic concentrations of the five assembly types after 5 years of cooling time. Those isotopes that showed a clearly visible distinction in the simulated spectra are highlighted. Note how the ${ }^{110 \mathrm{~m}} \mathrm{Ag}$ concentrations are now in the low parts per billion range, yet ${ }^{110 \mathrm{~m}} \mathrm{Ag}$ is still a distinctive feature of the gamma spectra because of its strong gamma emissions per decay. 


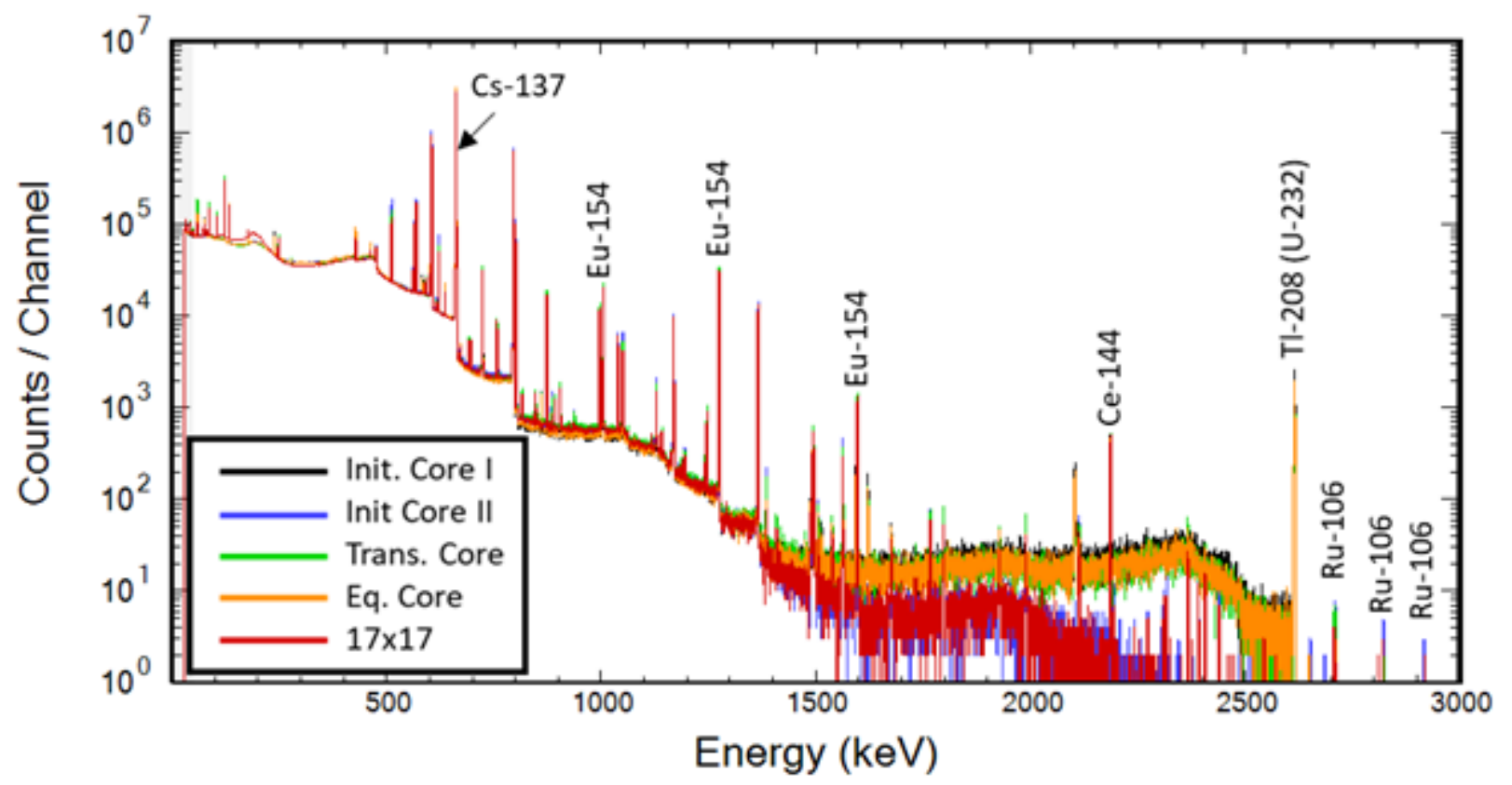

Figure 51. Simulated spectra of the five assembly types at 5 years after discharge from the reactor.

live-time $(s)=1000.00$

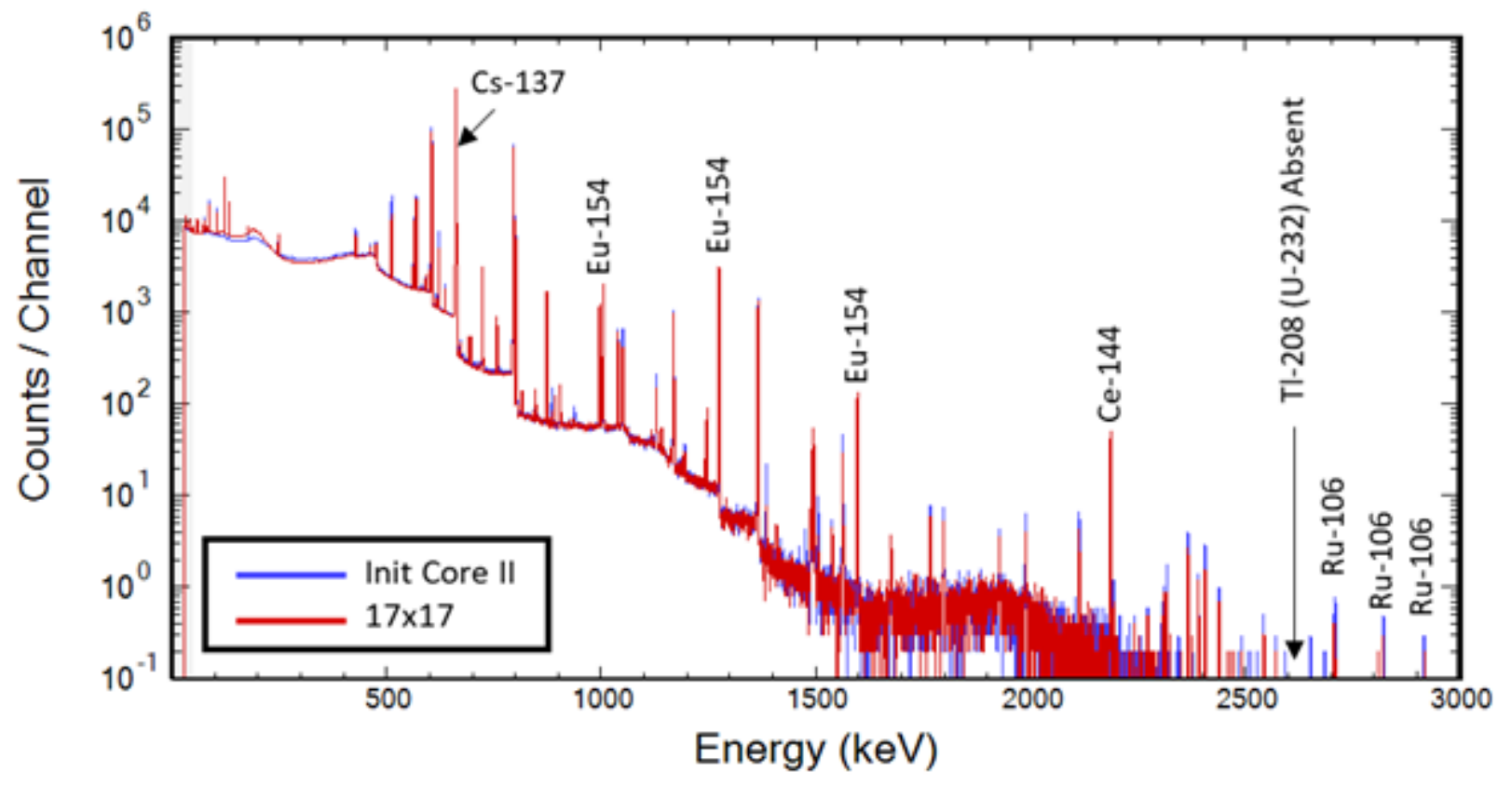

Figure 52. The simulated spectra of the two nonthorium assemblies at 5 years of cooling time. 
live-time $(\mathbf{s})=1000.00$

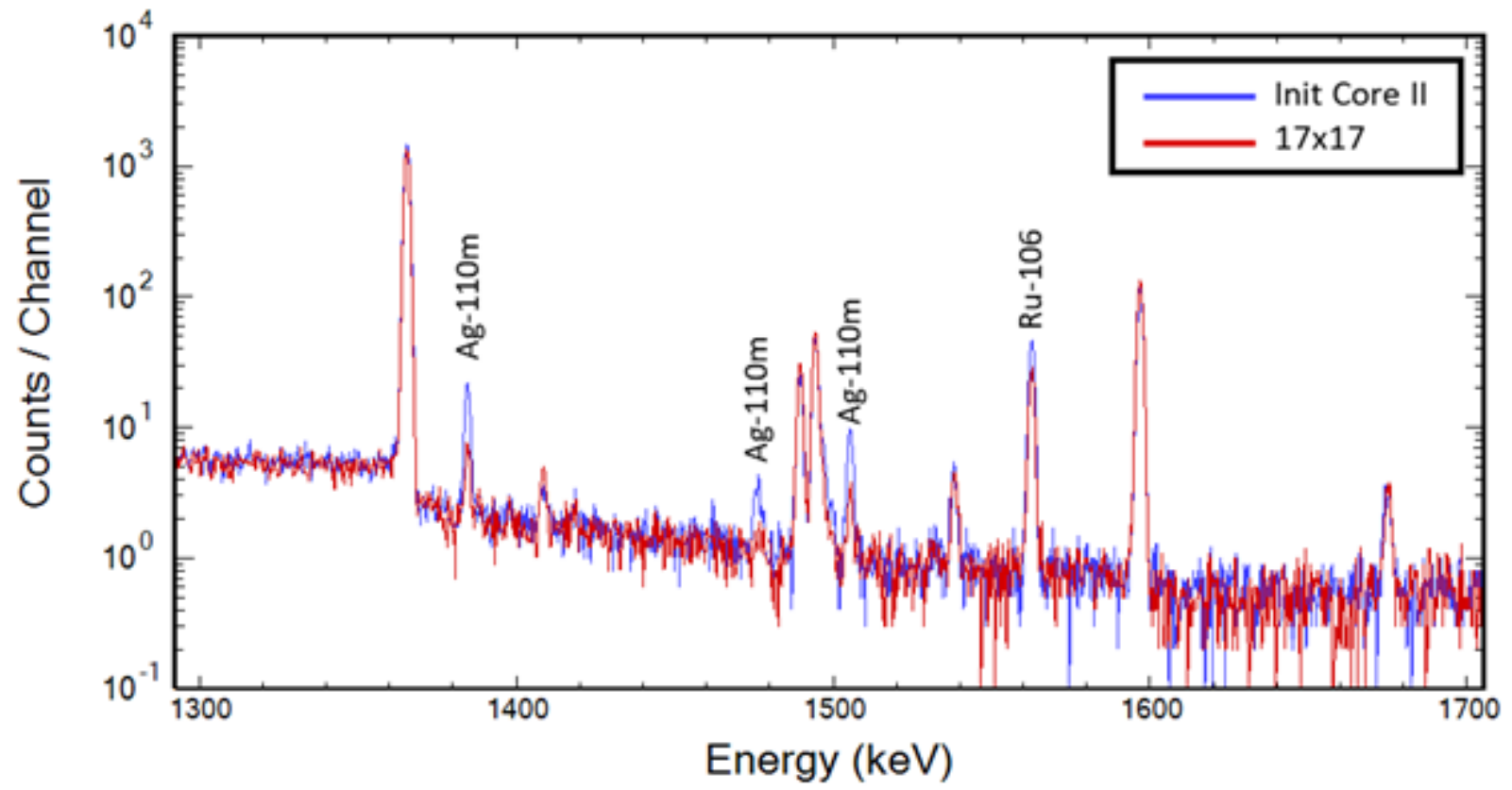

Figure 53. The 1300-1700 keV region of the simulated spectra for the two nonthorium assemblies at 5 years of cooling time.

live-time $(s)=10000$

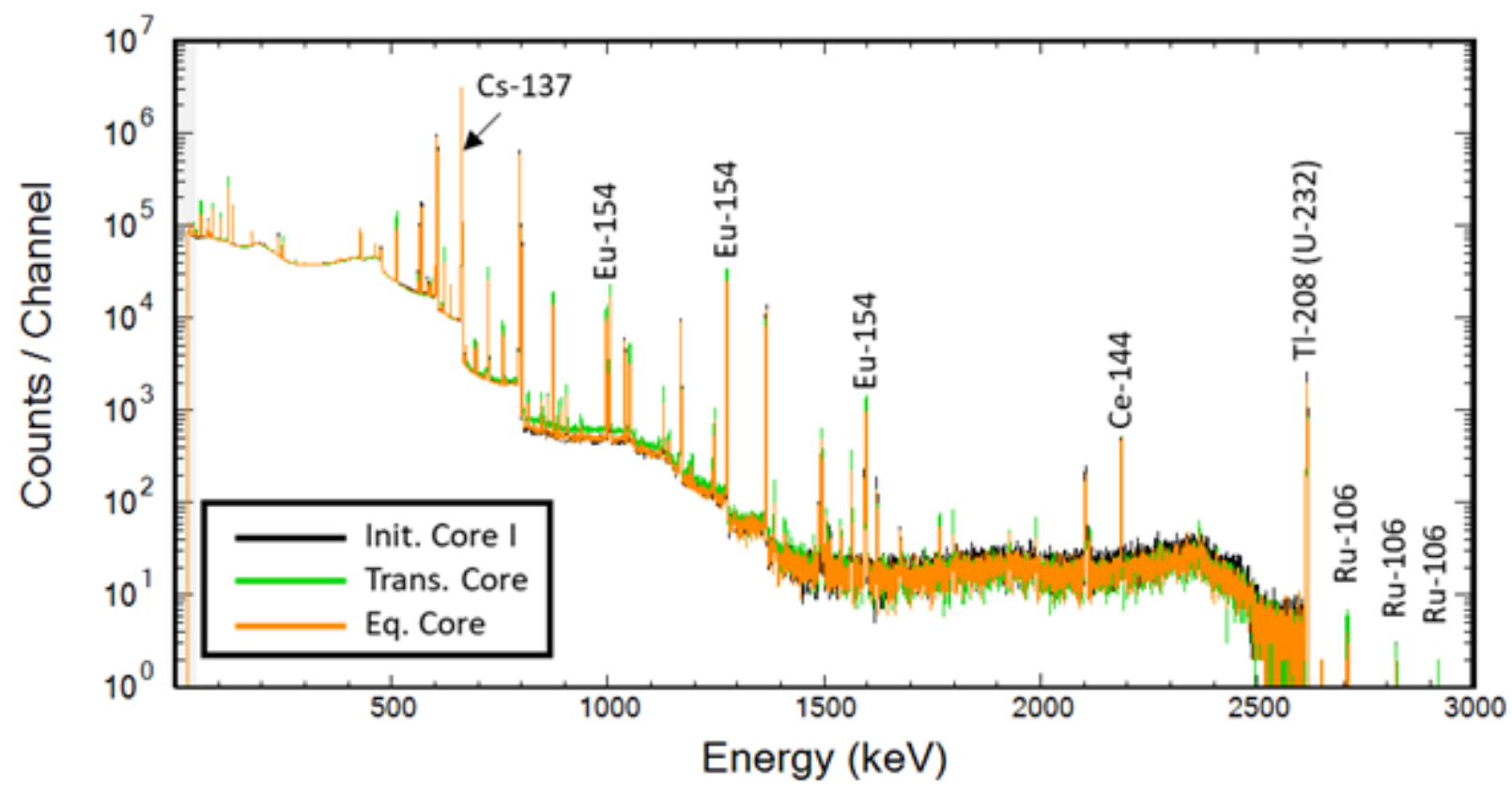

Figure 54. Simulated spectra of the three thorium-MOX assemblies at 5 years of cooling time. 


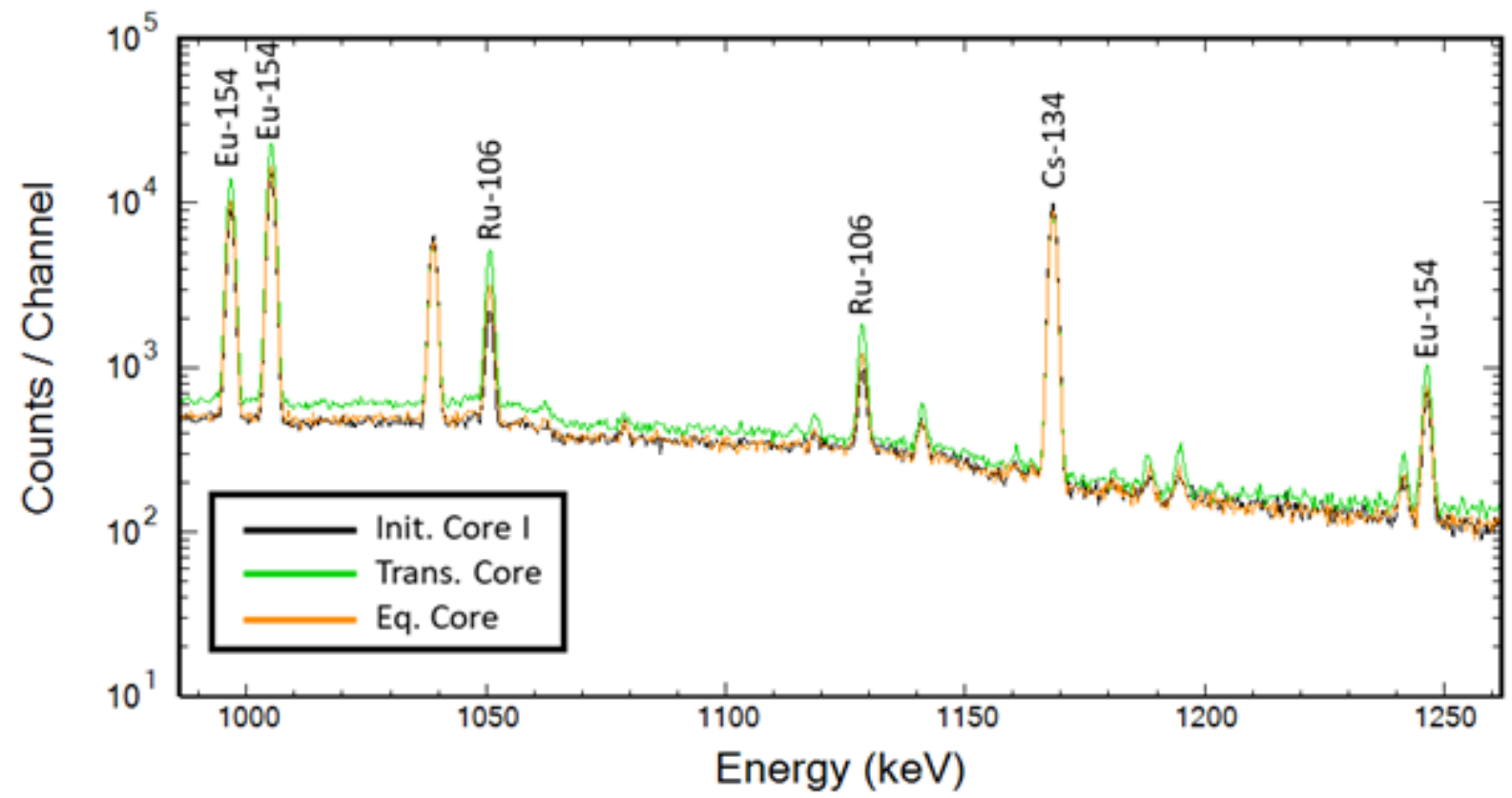

Figure 55. The 1000-1250 keV region of the simulated spectra of the three thorium-MOX assemblies at 5 years of cooling time.

Table 27. Isotopic concentrations (ppm) of the isotopes that could be identified from the simulated 5-year cooling time gamma spectra. Isotopic concentrations were taken directly from ORIGEN concentration files.

\begin{tabular}{|l|c|c|c|c|c|}
\multicolumn{5}{|c}{ Initial Core I Initial Core II } & \multicolumn{3}{c|}{ Transition Core } & Equilibrium Core & $\mathbf{1 7 \times 1 7}$ \\
\hline${ }^{110 \mathrm{~m}} \mathrm{Ag}$ & 0.011 & 0.025 & 0.015 & 0.009 & 0.006 \\
\hline${ }^{241} \mathrm{Am}$ & 144.301 & 287.714 & 1197.628 & 577.563 & 386.641 \\
\hline${ }^{144} \mathrm{Ce}$ & 3.992 & 3.259 & 3.647 & 3.887 & 3.872 \\
\hline${ }^{134} \mathrm{Cs}$ & 39.259 & 44.363 & 33.711 & 34.280 & 39.047 \\
\hline${ }^{137} \mathrm{Cs}$ & 2194.812 & 2136.073 & 2170.804 & 2231.705 & 2110.285 \\
\hline${ }^{154} \mathrm{Eu}$ & 21.465 & 28.204 & 32.788 & 23.479 & 30.689 \\
\hline${ }^{155} \mathrm{Eu}$ & 6.109 & 8.987 & 8.466 & 6.263 & 6.746 \\
\hline${ }^{85} \mathrm{Kr}$ & 91.740 & 30.397 & 57.368 & 93.259 & 52.581 \\
\hline${ }^{106} \mathrm{Ru}$ & 3.787 & 13.951 & 9.566 & 5.655 & 8.130 \\
\hline${ }^{125} \mathrm{Rb}$ & 5.910 & 5.365 & 5.556 & 6.065 & 4.163 \\
\hline${ }^{232} \mathrm{U}$ & 44.709 & 0.000 & 32.105 & 33.568 & 0.002 \\
\hline
\end{tabular}

\subsubsection{Twenty-year Spectra}

Figure 56 shows the simulated gamma spectra for the five assembly types after 20 years of cooling time. The three thorium-MOX assemblies are clearly distinguishable by their $2615 \mathrm{keV}$ peaks. After 20 years, all the ${ }^{232} \mathrm{U}$ daughters are in secular equilibrium. Unless the fuel is disturbed (e.g., by reprocessing), these peaks will remain in the spectrum for centuries until the ${ }^{232} \mathrm{U}$ (with a $70-\mathrm{yr}$ 
half-life) decays away completely. Figure 57 shows the spectra for the two nonthorium assemblies ( $17 \times 17$ and Initial Core II). After 20 years of cooling time, there are few clearly visible differences between the two assembly types. Careful measurements would still identify differences in peak areas of isotopes such as ${ }^{154} \mathrm{Eu}$. In Figure 58, the three thorium-MOX spectra are more clearly distinguishable. The largest differences are in peaks of the ${ }^{232} \mathrm{U}$ daughters, ${ }^{208} \mathrm{Tl}$ and bismuth-212 $\left({ }^{212} \mathrm{Bi}\right)$.

$$
\text { live-time }(s)=10000
$$

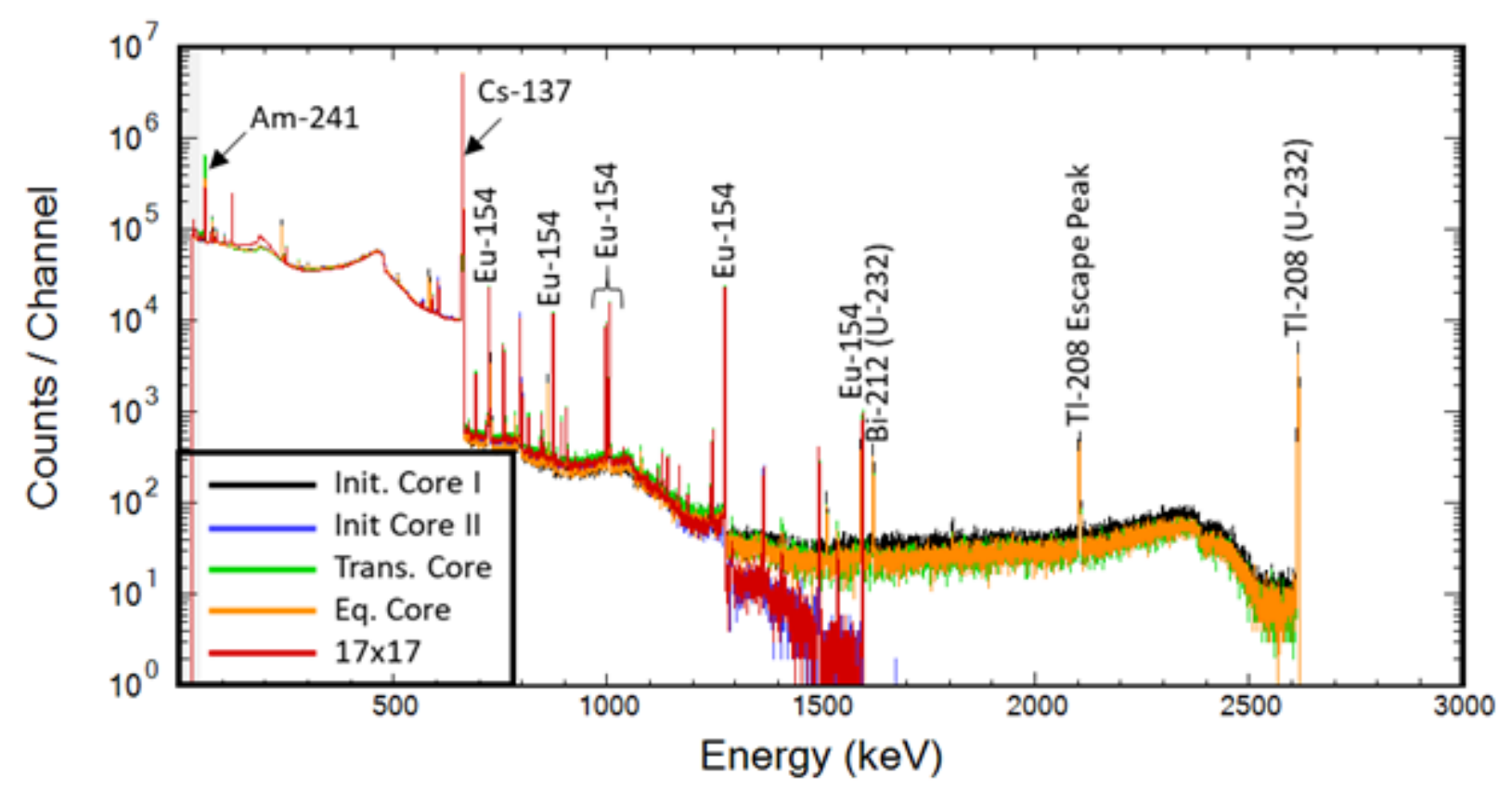

Figure 56. Simulated spectra of the five assembly types at 20 years after discharge from the reactor.

Table 28 shows the isotopic concentrations for the five assembly types after 20 years of cooling time. These are all the isotopes that could be detected from the gamma spectrum. Note how few isotopes are still visible after this much cooling time. Of the ones that remain, ${ }^{241} \mathrm{Am}$ and ${ }^{155} \mathrm{Eu}$ have low-energy peaks that probably could not be detected in an NDA measurement. Krypton-85 $\left({ }^{85} \mathrm{Kr}\right)$ has one strong peak at $514 \mathrm{keV}$, but its proximity to the $511 \mathrm{keV}$ annihilation peak makes it difficult to measure. Some antimony-125 $\left({ }^{125} \mathrm{Sb}\right)$ peaks are still detectible, but the 2.8-year half-life will make them increasingly difficult to detect at cooling times greater than 20 years. For the nonthorium assemblies, this leaves only ${ }^{154} \mathrm{Eu},{ }^{137} \mathrm{Cs}$, and ${ }^{134} \mathrm{Cs}$, which becomes weak due to its 2.1 -year half-life. For the thorium-MOX fuels, the ${ }^{232} \mathrm{U}$ daughter products add several very strong peaks that can be used to quantify the concentration of this isotope in the SNF. 
live-time $(s)=1000.00$

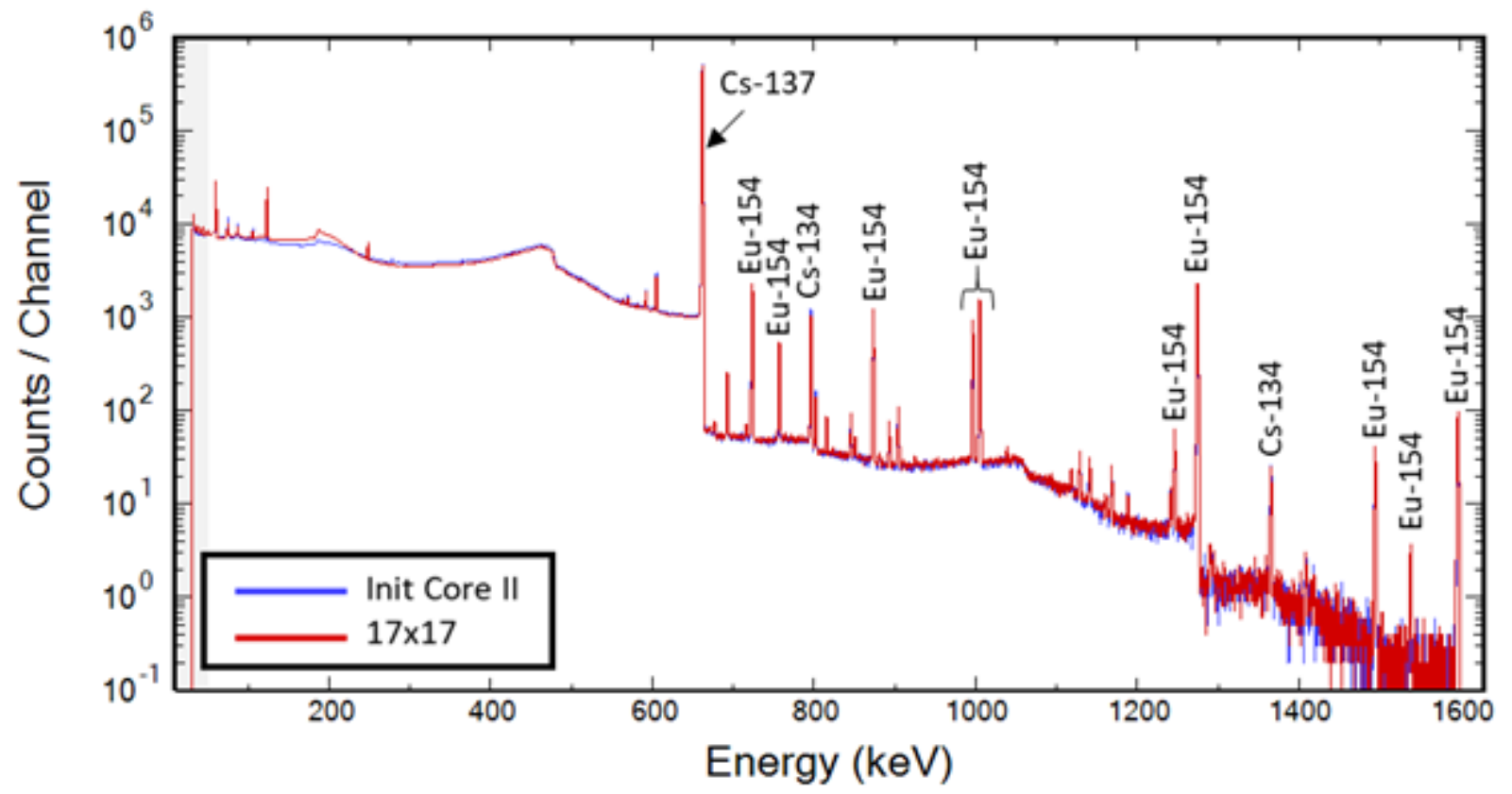

Figure 57. The simulated spectra of the two nonthorium assemblies at 20 years of cooling time.

live-time $(s)=10000$

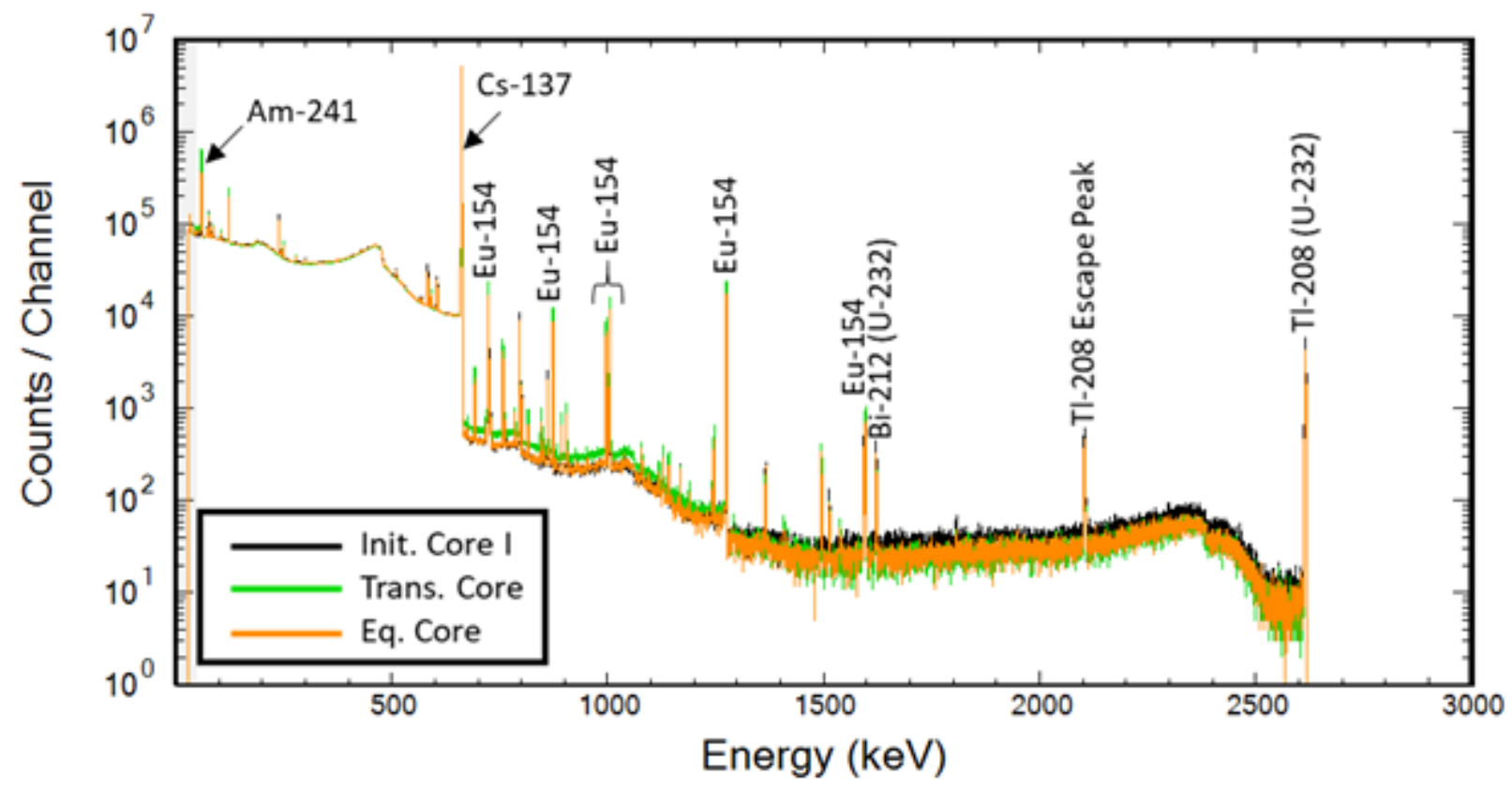

Figure 58. Simulated spectra of the three thorium-MOX assemblies at 20 years of cooling time. 
Table 28. Isotopic concentrations (ppm) of the isotopes that could be identified from the simulated 20-year cooling time gamma spectra. Isotopic concentrations were taken directly from ORIGEN concentration files.

\begin{tabular}{|c|c|c|c|c|c|}
\hline & Initial Core I & Initial Core II & Transition Core & Equilibrium Core & $17 \times 17$ \\
\hline${ }^{241} \mathrm{Am}$ & 332.115 & 729.364 & 2583.434 & 1278.403 & 945.830 \\
\hline${ }^{134} \mathrm{Cs}$ & 0.256 & 0.289 & 0.219 & 0.223 & 0.254 \\
\hline${ }^{137} \mathrm{Cs}$ & 1553.294 & 1511.723 & 1536.276 & 1579.429 & 1493.464 \\
\hline${ }^{154} \mathrm{Eu}$ & 6.408 & 8.419 & 9.788 & 7.009 & 9.161 \\
\hline${ }^{155} \mathrm{Eu}$ & 0.685 & 1.008 & 0.950 & 0.703 & 0.757 \\
\hline${ }^{85} \mathrm{Kr}$ & 34.891 & 11.561 & 21.818 & 35.469 & 19.998 \\
\hline${ }^{125} \mathrm{Sb}$ & 0.136 & 0.124 & 0.128 & 0.140 & 0.096 \\
\hline${ }^{232} U$ & 38.445 & 0.000 & 27.606 & 28.865 & 0.002 \\
\hline
\end{tabular}

\subsection{Key Observations}

In the PWR thorium fuel design with a heterogeneous two-region lattice, there is spatial separation of the driver fuel from the blanket; that is, the inner region (referred to as the seed region) contains only $\mathrm{UO}_{2}$ driver fuel, while the outer region (referred to as the blanket region) contains $\mathrm{UO}_{2}-\mathrm{ThO}_{2} \mathrm{MOX}_{\text {fuel. }}$ The spatial separation of the driver fuel from the blanket could potentially present a safeguards measurement challenge because there will be fissile material present in the inner region that could be shielded by the blanket fuel outer region. This would result in self-shielding of the item. Furthermore, direct detection of ${ }^{233} \mathrm{U}$ gamma rays will be a challenge for current safeguards measurement analysis because of the presence of ${ }^{232} \mathrm{U}$ progeny, as well as the presence of shielding. Further discussion of this issue is provided in Section 3. Other potential signatures, including ${ }^{233} \mathrm{Pax}$-rays and gamma rays, and other isotopes of protactinium that may be worthy of further exploration depending on the specific assay scenario, item form, and measurement configuration, were highlighted in the tables in this section.

The dose-rate comparisons between LWR SNF assemblies for uranium- and thorium-based fuels indicate that the differences between these cases likely do not warrant special R\&D for thorium fuel cycle applications, and their instrumentation challenges-in terms of the ability of detector instrumentation and data acquisition electronics to handle the high-radiation environment and acquire as well as process high count rates-will be similar for both conventional U/Pu and thorium fuel cycles. 


\section{Assessment of Thorium Fuel Cycle Safeguards Relevant Radiation Signatures}

This section focuses primarily on the assessment of radiation-based signatures of a variety of nuclear material types and forms that are unique to thorium fuel cycles. We evaluated whether thorium fuel cycle material, expected to be under safeguards (Section 1), generates signatures that can be readily measured with existing safeguards technologies, measured only if these technologies are appropriately modified, or whether no known, readily available technology for their measurement exists.

In general, safeguards measurements are performed for both nuclear material quantification (e.g., mass and activity) and characterization (e.g., elemental or isotopic composition), dictated by the fundamental properties of the radiation that produces individual signatures. As neutron radiation is highly penetrating, it enables assay of the entire bulk material of the measured item, yielding the mass of the item and thus quantitative information. Moreover, time-correlated neutrons are a unique signature of nuclear fission, which is an unambiguous signature of the presence of nuclear material. However, safeguards neutron measurements typically count all neutrons identically, with no attempt to distinguish between their energies by neutron spectroscopy. Furthermore, because the energies of the individual neutron emitted from different isotopes are not unique to the isotope of origin and their origins are thus indistinguishable, neutron counting alone cannot provide the isotopic composition of the assayed material. Despite its limited penetrability, gamma radiation is unique to the individual isotopes (i.e., its energy spectrum); therefore, it enables qualitative analysis of the assayed item and determination of its relative isotopic composition. Therefore, the combination of neutron and gamma signatures is usually required to provide complete information about the item of interest. In certain scenarios, knowledge of one or the other piece of information or process can be assumed, and only certain attributes of the item need to be verified.

Considering the different nature of neutron and gamma radiation, as well as the underlying technologies required for their detection, Section 3.1 addresses neutron signatures, modes and feasibility of NDA, and ENMC, AWCC, and MCNP simulations. Section 3.2 provides key observations and findings related to neutron signatures. Section 3.3 discusses gamma signatures. Section 3.4 provides key observations and findings related to gamma signatures. Section 3.5 addresses DA techniques for use with thorium fuel cycles.

\subsection{Neutron Signatures}

\subsubsection{Basic Neutron and Fission Nuclear Data Review}

Regardless of the origin of any nuclear material, or the fuel cycle process it has been part of, its safeguards signatures depend on the most fundamental physical properties (i.e., nuclear and atomic structure) determined by its elemental and isotopic composition. Note that these signatures can be affected by the surrounding material or material within the item itself (i.e., the matrix) and any item container or shielding. Hence, a brief neutron and fission nuclear data review is provided, focusing on the high-level differences between properties of isotopes typically, but not exclusively, associated 
with U/Pu fuel cycles and those unique to thorium fuel cycles. The nuclear data presented below are, unless noted otherwise, retrieved from or based on the ENDF/B-VIII.0 (Evaluated Nuclear Data File, version 8), evaluated nuclear data library [49], which is also used in MCNP calculations discussed further in this section. While other evaluated nuclear data libraries are available to the safeguards community (e.g., JENDL-4.0 [Japanese Evaluated Nuclear Data Library] [50]), we do not attempt to draw conclusions about which database is superior and generally consider the differences as negligible in the context of the main objectives of this scoping study. Similarly, we assume that the uncertainty associated with individual evaluated nuclear data values is second order for the purpose of measurement concept development, and its explicit evaluation is recommended for future work.

Traditionally, for safeguards measurements using passive neutron counting, the most important physical properties of individual fissile or fertile isotopes have been spontaneous fission (SF) multiplicity and neutron yield, as they determine the intensity of the neutrons that are emitted spontaneously from the item and are available for detection. Table 29 lists SF yields and multiplicities for select isotopes relevant for both U/Pu and thorium fuel cycles. The SF multiplicities are of similar magnitude for all relevant isotopes. While significant for other applications, including nuclear reactor physics and criticality safety, differences in these values are of little importance in the context of the feasibility of safeguards measurements, where discrete items containing quantities of nuclear material on the order of hundreds of grams to a few kilograms are of interest. SF yields are determined by the stability of nuclei of individual isotopes (i.e., half-life) and the branching ratio between the most dominant decay mode (i.e., $\alpha$-decay) and SF. Hence, the SF yields, calculated for this work based on recommended values of SF half-life reported in Ref. [51], differ dramatically among the isotopes listed in Table 29. In general, except for even isotopes of Pu, SF yields are simply too low to be of any practical value in the case of the fissile isotopes ${ }^{233} \mathrm{U},{ }^{235} \mathrm{U}$, and ${ }^{239} \mathrm{Pu}$. For example, a significant quantity of ${ }^{233} \mathrm{U}$ would emit only approximately three SF neutrons per second, while the same amount of reactor-grade $\mathrm{Pu}\left(\sim 40 \%{ }^{240} \mathrm{Pu}\right)$ would produce about 3 million times more neutrons. 
Table 29. Spontaneous fission multiplicities and fission yields of selected thorium, uranium, plutonium, and americium isotopes [52].

\begin{tabular}{|c|c|c|c|c|c|}
\hline Nuclide & Half-life & SF multiplicity [48] & \begin{tabular}{|l} 
SF yield \\
$\left(n \cdot s^{-1} \cdot g^{-1}\right)$
\end{tabular} & $\begin{array}{l}\text { Alpha yield } \\
\left(\alpha \cdot s^{-1} \cdot g^{-1}\right)\end{array}$ & $(\alpha, n)$ yield in oxide $\left[n \cdot s^{-1} \cdot g^{-1}\right.$ \\
\hline${ }^{232} \mathrm{Th}$ & $1.41 \times 10^{10}$ years & 2.14 & $>6 \times 10^{-8}$ & $4.1 \times 10^{3}$ & $2.2 \times 10^{-5}$ \\
\hline${ }^{232} \mathrm{U}$ & 71.7 years & 1.71 & $1.3 \times 10^{0}$ & $8.0 \times 10^{11}$ & $1.5 \times 10^{4}$ \\
\hline${ }^{233} \mathrm{U}$ & $1.59 \times 10^{5}$ years & 1.76 & $8.6 \times 10^{-4}$ & $3.5 \times 10^{8}$ & 4.8 \\
\hline${ }^{234} \mathrm{U}$ & $2.45 \times 10^{5}$ years & 1.81 & $5.02 \times 10^{-3}$ & $2.3 \times 10^{8}$ & 3.0 \\
\hline${ }^{235} \mathrm{U}$ & $7.04 \times 10^{8}$ years & 1.86 & $2.99 \times 10^{-4}$ & $7.9 \times 10^{4}$ & $7.1 \times 10^{-4}$ \\
\hline${ }^{238} \mathrm{U}$ & $4.468 \times 10^{9}$ years & 2.01 & $1.36 \times 10^{-2}$ & $1.2 \times 10^{4}$ & $8.3 \times 10^{-5}$ \\
\hline${ }^{238} \mathrm{Pu}$ & 87.74 years & 2.21 & $2.59 \times 10^{3}$ & $6.4 \times 10^{11}$ & $1.3 \times 10^{4}$ \\
\hline${ }^{239} \mathrm{Pu}$ & $2.41 \times 10^{4}$ years & 2.16 & $2.18 \times 10^{-2}$ & $2.3 \times 10^{9}$ & $3.8 \times 10^{1}$ \\
\hline${ }^{240} \mathrm{Pu}$ & $6.56 \times 10^{3}$ years & 2.16 & $1.02 \times 10^{3}$ & $8.4 \times 10^{9}$ & $1.4 \times 10^{2}$ \\
\hline${ }^{241} \mathrm{Pu}$ & 14.35 years & 2.25 & $5 \times 10^{-2}$ & $9.4 \times 10^{7}$ & 1.3 \\
\hline${ }^{242} \mathrm{Pu}$ & $3.76 \times 10^{5}$ years & 2.15 & $1.72 \times 10^{3}$ & $1.4 \times 10^{8}$ & 2.0 \\
\hline${ }^{241} \mathrm{Am}$ & 433.6 years & 3.22 & $1.18 \times 10^{0}$ & $1.3 \times 10^{11}$ & $2.7 \times 10^{3}$ \\
\hline${ }^{252} \mathrm{Cf}$ & 2.646 years & 3.78 & $2.34 \times 10^{12}$ & $1.9 \times 10^{13}$ & $6.0 \times 10^{5}$ \\
\hline
\end{tabular}

Figures 59-68 display neutron-induced fission cross-sections of select thorium, uranium, plutonium, and americium isotopes as a function of incident neutron energy, as retrieved from the ENDF/B-VIII.0 evaluated nuclear data library [49]. From the perspective of isotopes prominent in thorium fuel cycles, it appears that ${ }^{232} \mathrm{Th}$ displays similar magnitude and energy dependence of the neutron-induced fission cross-section as ${ }^{238} \mathrm{U}$, while the ${ }^{233} \mathrm{U}$ neutron-induced fission cross-section magnitude and its dependency on incident neutron energy strongly resembles that of ${ }^{235} \mathrm{U}$. In other words, based on the fundamental nuclear data associated with each isotope alone, it is possible to draw the analogies that ${ }^{232} \mathrm{Th}$ and ${ }^{233} \mathrm{U}$ are qualitatively identical to ${ }^{238} \mathrm{U}$ and ${ }^{235} \mathrm{U}$, respectively, in terms of fission neutron signatures. Furthermore, this enables immediate identification of classes of already-established NDA methods that can or cannot be directly utilized or adapted for the assay of the most prominent thorium fuel cycle materials that are a given chemical or physical form of ${ }^{232} \mathrm{Th}$ or ${ }^{233} \mathrm{U}$.

\subsubsection{Modes of Neutron Nondestructive Assay}

NDA is performed to characterize the desired properties of the bulk measured item without altering its chemical or physical form. Traditionally, for nuclear safeguards, two distinct modes of NDA have been established: passive and active. A third mode of assay, self-interrogation, has been recently developed and is recognized and discussed in this report. 


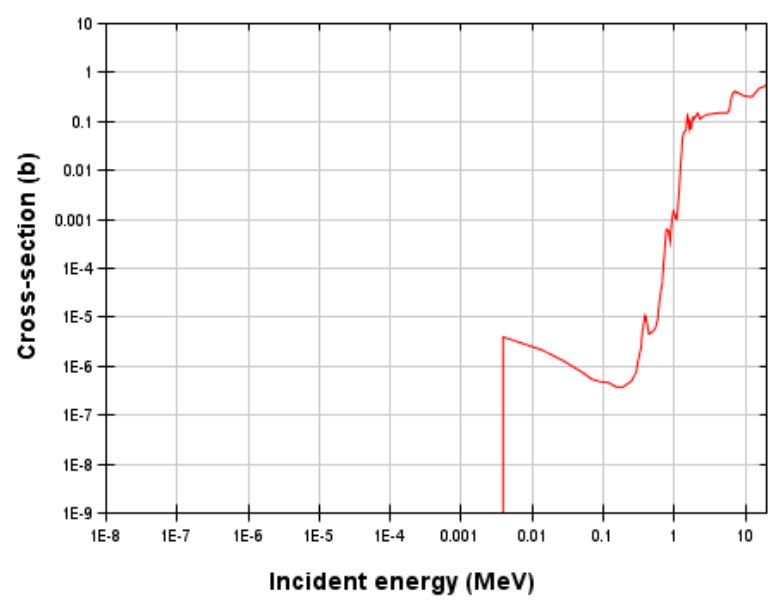

Figure 59. ${ }^{232}$ Th fission cross-section [49].

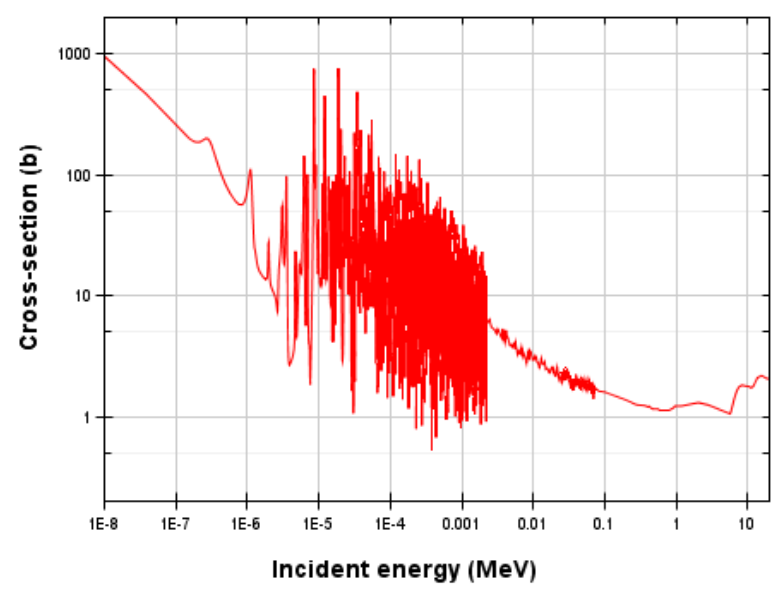

Figure $61 .{ }^{235} \mathrm{U}$ fission cross-section [49].

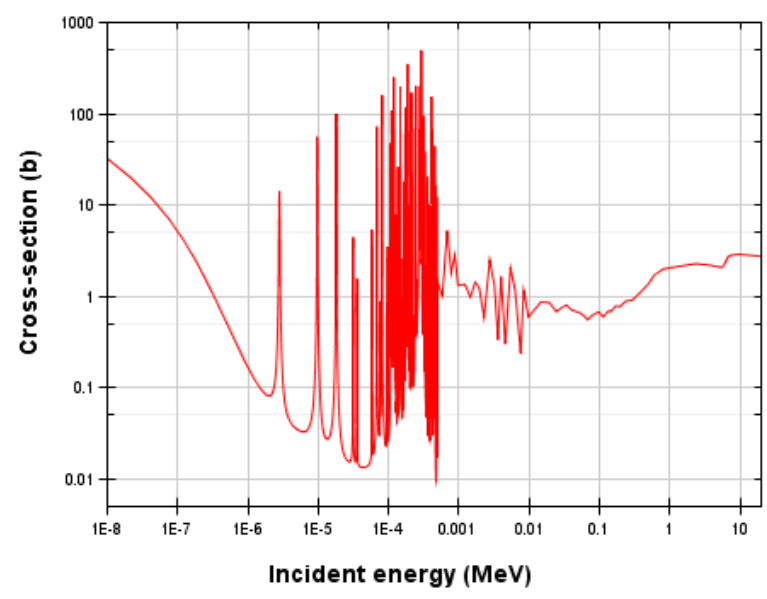

Figure $63 .{ }^{238} \mathrm{Pu}$ fission cross-section [49].

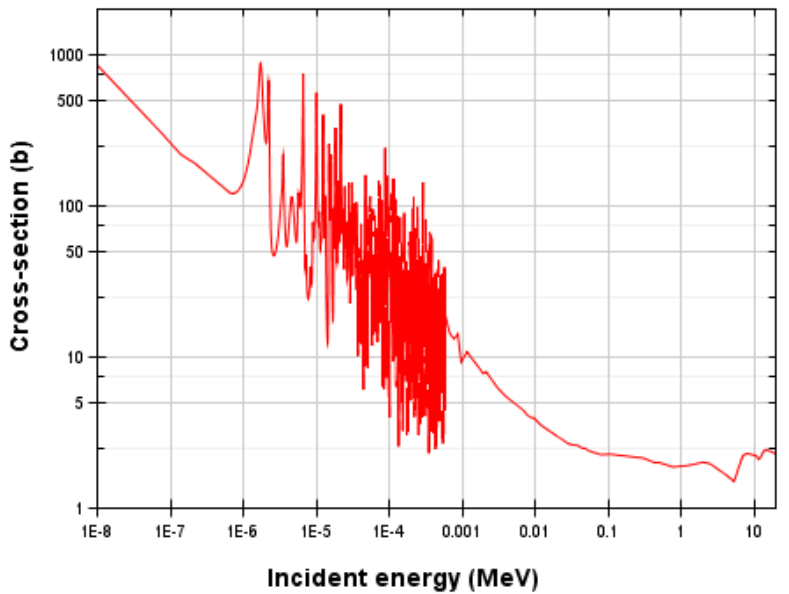

Figure $60 .{ }^{233} \mathrm{U}$ fission cross-section [49].

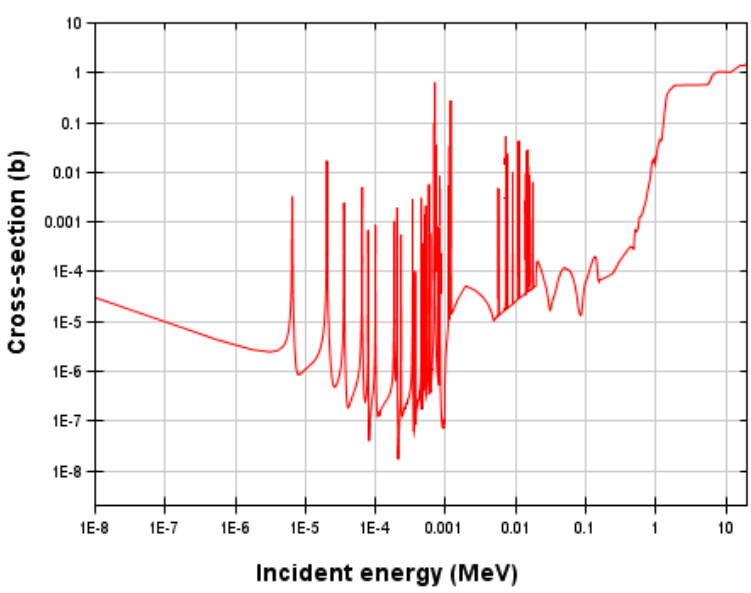

Figure $62 .{ }^{238} \mathrm{U}$ fission cross-section [49].

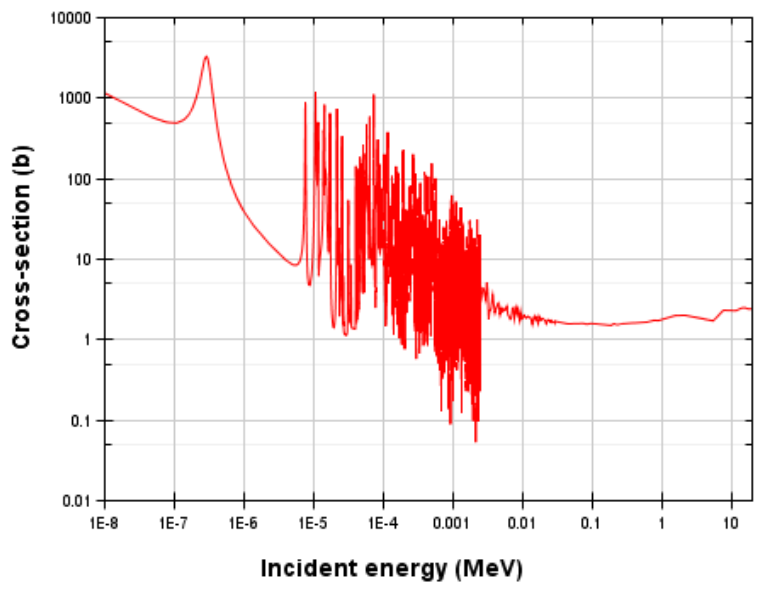

Figure $64 .{ }^{239} \mathrm{Pu}$ fission cross-section [49]. 


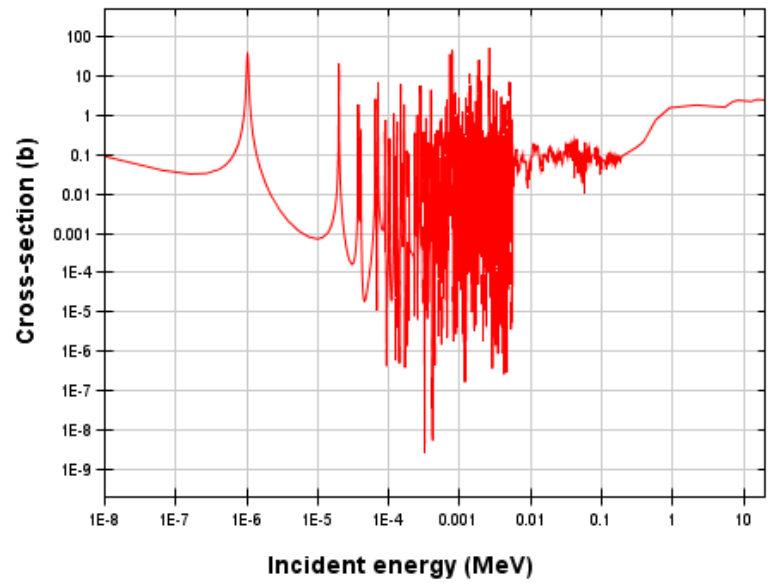

Figure 65. ${ }^{240} \mathrm{Pu}$ fission cross-section [49].

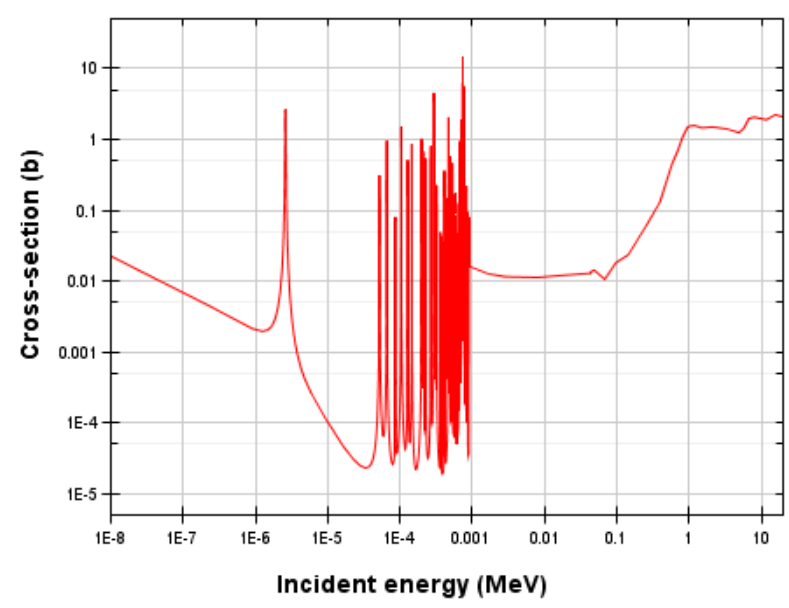

Figure 67. ${ }^{242} \mathrm{Pu}$ fission cross-section [49].

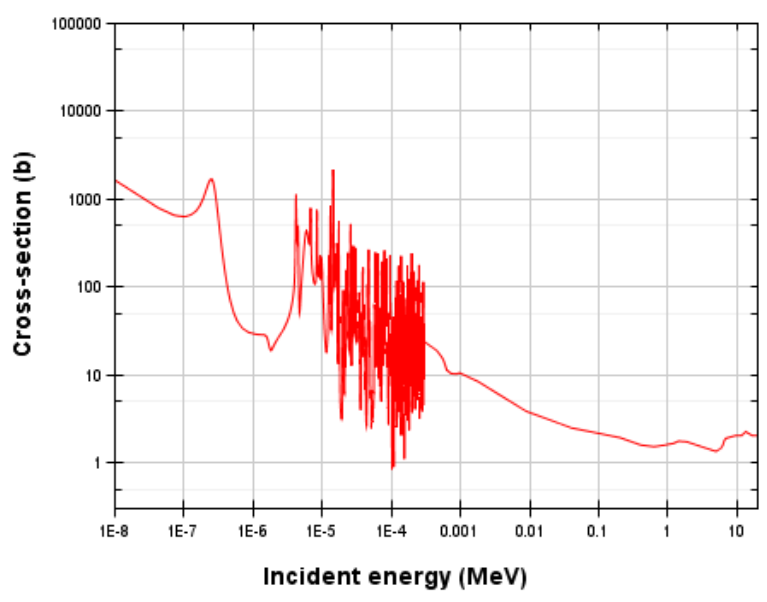

Figure 66. ${ }^{241} \mathrm{Pu}$ fission cross-section [49].

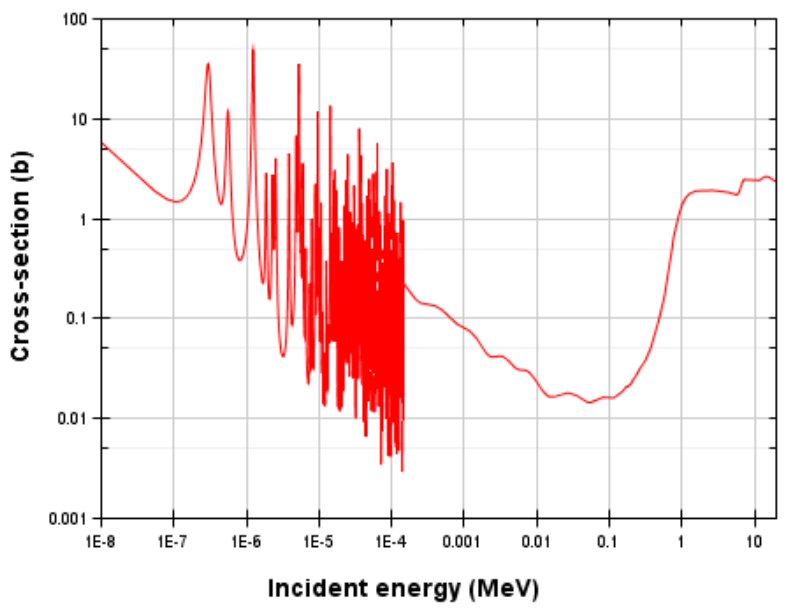

Figure 68. ${ }^{241} \mathrm{Am}$ fission cross-section [49].

\subsubsection{Passive Nondestructive Assay}

In passive NDA, the properties of the item may be assessed by detection and characterization of its spontaneous radiation emission (i.e., neutrons in the context of this section). The intensity of this radiation is nearly exclusively a function of the item's isotopic composition and cannot be controlled by a fuel cycle facility/reactor operator, except for the multiplication of the item, which depends on item and detector geometry that can be, to a degree, modified by the operator. However, this assumes that the physical properties of the item are such that the spontaneous (i.e., neutron) radiation is of sufficient intensity to be of practical value when quantified. Strictly speaking, all isotopes of thorium, uranium, and plutonium relevant in this report do emit neutrons from SF. However, the SF rate of many isotopes (primarily fissile ones) is impractically low, such that the SF neutron detection rates are too low to achieve statistically significant precision in the relatively short measurement time intervals ( 15-60 minutes for neutron counting) relevant for most nuclear safeguards applications. 
In addition to SF, many relevant thorium, uranium, and plutonium isotopes decay via a-decay. However, the range of $\alpha$-particles is too short $(\sim \mu \mathrm{m})$ to be of direct relevance when the material is bulk in nature because they cannot fully escape the measured item to be externally detected. Whereas, if the actinide atom is chemically bound to, or mixed in a matrix with, material containing low-Z (atomic number) elements, the emitted $a$-particle may be absorbed by the atom of the light element, leading to the emission of a single neutron via an $(\alpha, n)$ reaction. These $(\alpha, n)$ neutrons have energies similar to neutrons from SF and thus are indistinguishable on an event-by-event basis. Because these neutrons are not correlated in time with one another, it is possible to quantify their relative contribution to overall detected neutron rates by single (i.e., total) versus time-correlated neutron counting and the application of appropriate statistical methods.

\subsubsection{Active Nondestructive Assay}

In active NDA, the properties of the item are inferred from its induced response to some action of the operator/inspector, which is referred to as interrogation. In the context of the active assay of nuclear material for nuclear safeguards, the interrogation is traditionally performed by irradiation of the item by certain particles, whereby their selection and properties dictate the item's response and the quality of information that can be obtained. When considering the neutron signatures of nuclear material, the irradiation is typically performed by an external neutron interrogation source that may induce fission within the material, ultimately allowing assessment of the effective amount of all fissionable isotopes present. Depending on the nature of the item, different sources of neutron radiation may be better suited than others to extracting relevant fissile isotope characteristics. The three types of neutron sources below are traditionally utilized based on the properties of their emitted neutron radiation.

\section{1) Neutron generators}

Neutron generators (Figure 69) use deuterium-deuterium (D-D) and deuterium-tritium (D-T) reactions to produce monoenergetic neutrons with energies of $2.45 \mathrm{MeV}$ and $14 \mathrm{MeV}$, respectively. Neutron generators can operate in continuous or pulsed mode with COTS models able to produce total neutron intensities up to $\sim 1 \times 10^{9} \mathrm{n} / \mathrm{s}$. In comparison to other neutron sources, the main advantage of neutron generators is their ability to turn the neutron source, which includes a pulsed mode of operation, on and off as desired. The main disadvantage is the relatively complex instrumentation required for its operation leading to the requirement for personnel to be trained to a greater level of competence. Furthermore, a high cost and maintenance burden are incurred with pulsed systems [53]. In general, radiological protection measures must be in place when operating neutron generators due to the high neutron energies and intensities [54]. However, the high neutron interrogating flux does have the benefit of reducing measurement times and enabling measurement in a higher passive neutron background (e.g., spent nuclear fuels with high $\mathrm{Pu} / \mathrm{Cm}$ content) [53]. Neutron generators also have limited run time, depending on the manufacturer, prior to the need for target replacement.

\section{2) $(a, n)$ neutron sources}

Neutrons are produced by $(a, n)$ reactions in a source consisting of a mixture of a strong alpha emitter (e.g., Am or Pu) and a light element (e.g., lithium or beryllium). The elemental composition and geometry of the source, together with its enclosure, defines the energy spectrum of outgoing neutrons, which is typically softer than that of neutron generators, although the shape of the neutron spectrum and maximum energy may vary significantly for different $(a, n)$ neutron sources 
(Table 30 and Figure 70). Additionally, the produced neutrons are emitted randomly in time and are thus not correlated in time with one another; therefore, these neutrons can be statistically distinguished from time-correlated neutrons emitted from induced fissions (IFs). The main advantage of such a neutron source is its simplicity and reliability because it does not require any support electronics to produce neutron radiation. The main disadvantage is that it cannot be turned off; thus, shielding is required for transport or storage when not in use. It is also hard to transport radiation sources internationally for inspections. Furthermore, the half-life of isotopic neutron sources must also be considered, versus the run time of neutron generators, requiring them to be replaced.

\section{3) Spontaneous fission sources}

Neutrons produced by SF are correlated in time. The most common SF source used for NDA applications is californium-252 $\left({ }^{252} \mathrm{Cf}\right)$. The neutron energy distribution for SF corresponds to a Watt fission spectrum with mean neutron energy around $2 \mathrm{MeV}$, but with a significant high energy tail up to $10 \mathrm{MeV}$. Just as with $(\alpha, n)$ neutron sources, the main advantage of SF sources is their simplicity of operation, and the main limitation is the presence of continuous radiation, which may require shielding when not in use. Moreover, the half-life of ${ }^{252} \mathrm{Cf}$ is $\sim 2.6$ years; therefore, the operator/inspector must accommodate a significant decrease in neutron intensity over a period of time that is typically shorter than the lifetime of a given facility where this type of source will be employed. Hence, regular replacement of ${ }^{252} \mathrm{Cf}$ sources may be required for uninterrupted operations. Additionally, should an SF source be used for the active assay of fissile material in terms of counting real coincidences from IF, it may be significant to properly distinguish the contribution of the interrogating source from the response of the interrogated item $[55,56]$.

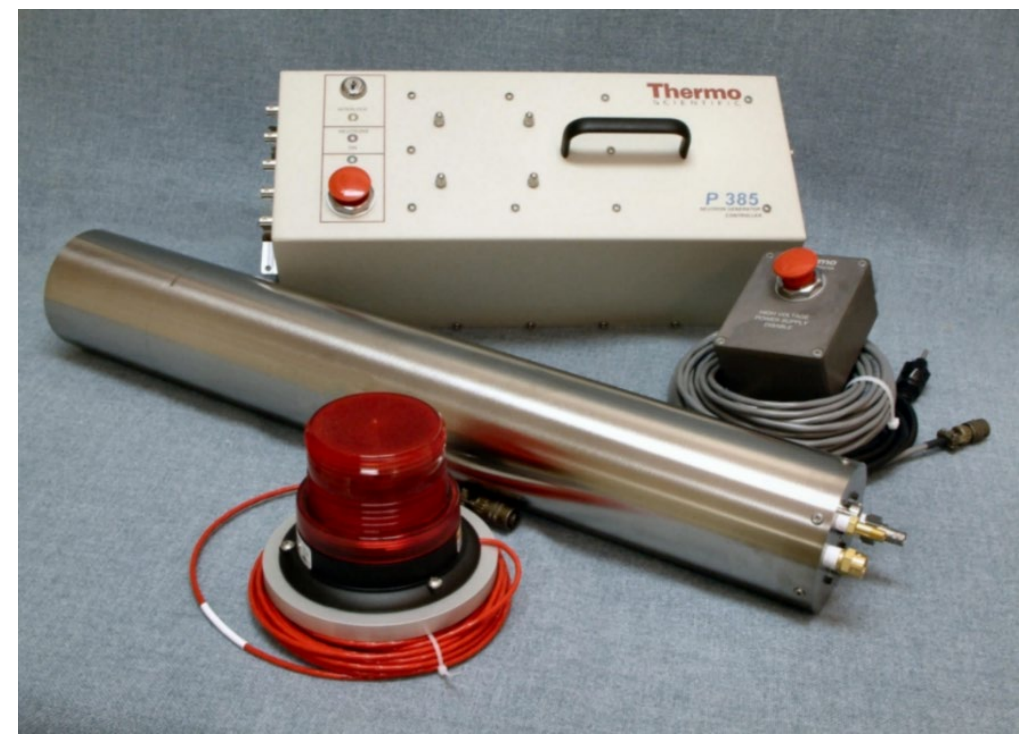

Figure 69. D-T neutron generator available from Thermo Scientific (model P385), including the electronics enclosure, emergency shutdown button module, accelerator tube, and indicator lamp [57]. 
Table 30. Examples of common alpha neutron sources [51].

\begin{tabular}{|l|c|c|}
\hline Alpha neutron source* & Average neutron energy (MeV) & Maximum neutron energy (MeV) \\
\hline${ }^{241} \mathrm{AmBe}$ & 5.0 & 11.0 \\
\hline${ }^{241} \mathrm{AmLi}$ & 0.3 & 1.5 \\
\hline${ }^{241} \mathrm{AmB}$ & 2.8 & 5.0 \\
\hline${ }^{241} \mathrm{AmF}$ & 1.3 & 2.5 \\
\hline${ }^{238} \mathrm{PuBe}$ & 4.5 & 11.0 \\
\hline${ }^{239} \mathrm{PuBe}$ & 4.5 & 10.7 \\
\hline${ }^{226} \mathrm{RaBe}$ & 4.3 & 10.4 \\
\hline
\end{tabular}

*The intensities of these isotopic sources scale with the decay of the driving isotope.

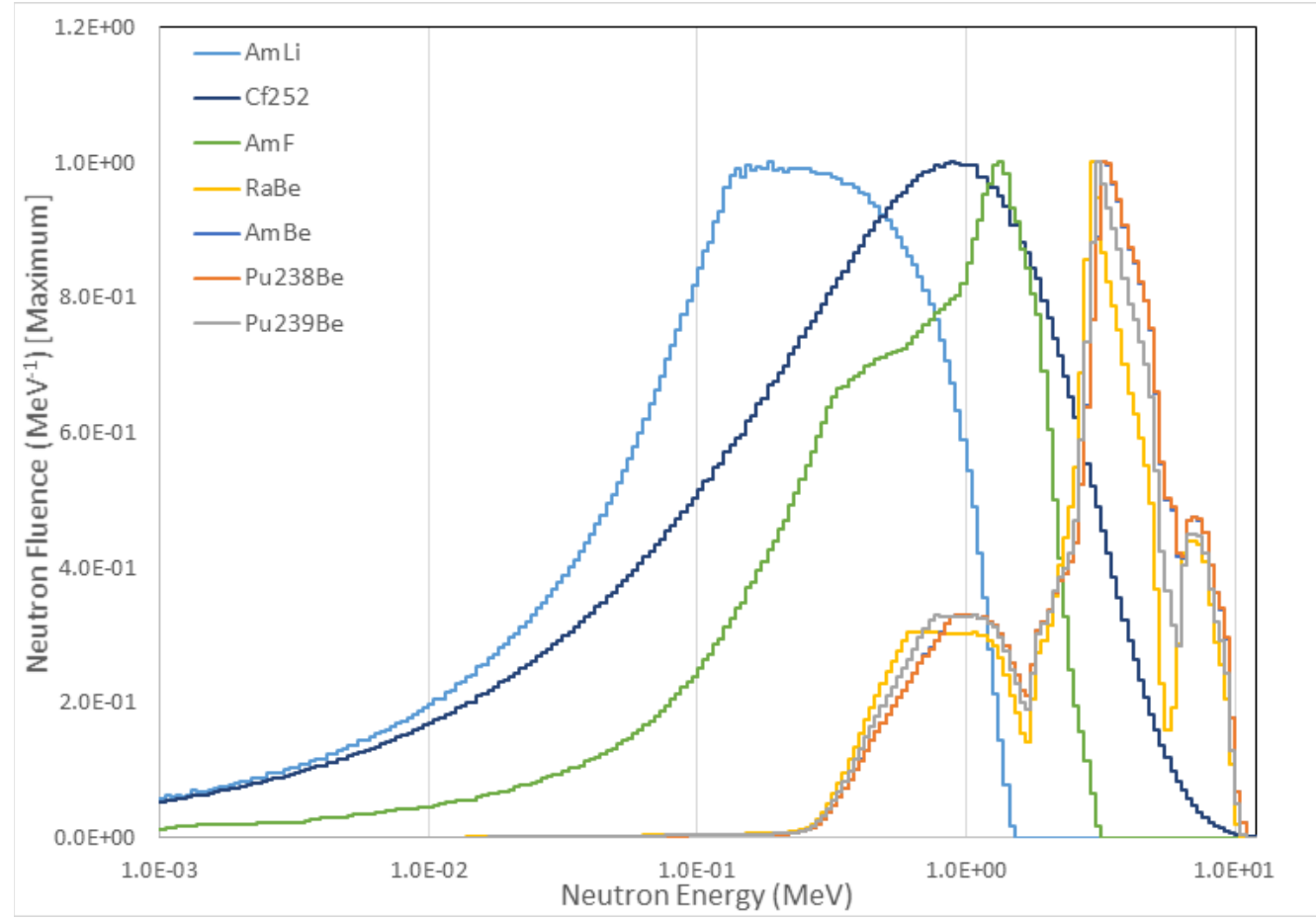

Figure 70. Energy spectrum of neutrons created in various isotopic sources by $(\alpha, n)$ reactions or spontaneous fission (simulations using GADRAS code [46]) to show the dynamic neutron energy range of available interrogation sources.

\subsubsection{Self-Interrogation}

Self-interrogation [58] is a special mode of NDA that combines certain aspects of both passive and active assay modes. From the perspective of the operator/inspector, self-interrogation is a passive mode of assay because it does not require the introduction of external radiation (or an external interrogation source) to induce a response from the item. From the perspective of the item, it is similar to active assay in the sense that a response is being induced within the item; however, the interrogating source radiation is internal to the item itself. An example of self-interrogation is the differential die-away self-Interrogation (DDSI) technique applied to the assay of Pu content in SNF $[59,60]$, where fission neutrons from the SF of several isotopes escape from individual fuel pins and 
enter the water surrounding the fuel assembly. Note that the exact isotopic composition depends on the starting composition and irradiation history of the fuel but typically includes curium $-244\left({ }^{244} \mathrm{Cm}\right)$ and ${ }^{240} \mathrm{Pu}$. The neutrons are then thermalized and reflected into the individual pins. As their energy decreases to thermal levels, these neutrons will preferentially induce fission of fissile isotopes within the pins (i.e., ${ }^{235} \mathrm{U}$ and ${ }^{239} \mathrm{Pu}$ ), and not ${ }^{240} \mathrm{Pu}$ or ${ }^{244} \mathrm{Cm}$ from which they originated. Another example is the self-interrogation of uranium hexafluoride $\left(\mathrm{UF}_{6}\right)$ in $30 \mathrm{~B}$ and $48 \mathrm{Y}$ cylinders $[61,62]$. In this case, trace levels of the intense alpha-emitter ${ }^{234} \mathrm{U}$ cause enough $(\alpha, \mathrm{n})$ reactions on fluorine-19 $\left({ }^{19} \mathrm{~F}\right)$ present within the item that the resulting neutron flux can induce fission on ${ }^{235} \mathrm{U}$, leading to measurable rates of correlated fission neutrons. In both examples, as well as other self-interrogation scenarios, the efficacy of self-interrogation depends on the geometrical configuration of the item (e.g., the DDSI technique would not work without water between the fuel pins, which thermalizes and reflects the neutrons back into those fuel pins) or its chemical composition. For example, only the presence of a sufficient amount of low-Z element-fluorine, in this case-allows $\alpha$-particles from the $\alpha$-decay of ${ }^{234} \mathrm{U}$ to create a sufficiently strong interrogating neutron flux to result in a measurable signal from the IF of

${ }^{235} \mathrm{U}$. Thus, self-interrogation does not depend exclusively on the fundamental properties of the nuclear material, but also on external "engineering" factors under the control of the operator/inspector and, therefore, in the context of this report, warrants a separate set of considerations from passive and active NDA.

\subsubsection{Feasibility of ${ }^{232} \mathrm{Th}$ and ${ }^{233} \mathrm{U}$ Neutron Nondestructive Assay}

This section discusses the feasibility of the NDA of two isotopes that are unique to thorium-based nuclear fuel cycles- ${ }^{232} \mathrm{Th}$ and ${ }^{233} \mathrm{U}$-based on fundamental principles. For each of these isotopes, a high-level evaluation is provided regarding the feasibility of NDA using passive, active, or selfinterrogating techniques. This evaluation is then used to inform future research recommendations, which are presented in Section 5.

\subsubsection{Thorium-232}

As discussed in Section 1, ${ }^{232}$ Th can be found across thorium-based fuel cycles of consideration in multiple chemical and physical forms, including $\mathrm{ThO}_{2},\left({ }^{\text {nat }} \mathrm{U}, \mathrm{Th}\right) \mathrm{O}_{2},\left({ }^{233} \mathrm{U}, \mathrm{Th}\right) \mathrm{O}_{2}$, (enriched $\left.\mathrm{U}, \mathrm{Th}\right) \mathrm{O}_{2}$, ThC, Th metal, and Th(FLiBe) salt.

\section{Passive nondestructive assay}

As shown in Table 29, the SF rate of ${ }^{232} \mathrm{Th}$ is extremely low at $1.02 \times 10^{-7} \mathrm{n} / \mathrm{g} \cdot \mathrm{s}$. It would take $\sim 20$ metric tons of ${ }^{232}$ Th to yield, on average, one SF per second. It is thus straightforward to conclude that no neutron-based passive technique can reach the level of practicality necessary for successful deployment for nuclear safeguards applications.

\section{Active nondestructive assay}

${ }^{232} \mathrm{Th}$ is a fissionable material with a fission threshold around $1.5 \mathrm{MeV}$. Figures 59 and 62 enable a direct comparison of the neutron-induced fission cross-sections of ${ }^{232} \mathrm{Th}$ and ${ }^{238} \mathrm{U}$, which are quantified in Table 31 for specific ranges of neutron energies. As already discussed, and as evident from the figures and table, ${ }^{232} \mathrm{Th}$ exhibits qualitatively similar behavior to ${ }^{238} \mathrm{U}$. Thus, collective experience with NDA of ${ }^{238} \mathrm{U}$ is in principle applicable to ${ }^{232} \mathrm{Th}$ and can be summarized by these general principles: 
- Thermal neutrons are NOT suitable for the assay of ${ }^{232} \mathrm{Th}$.

- Only fast neutrons with energies above $1.5 \mathrm{MeV}$ can yield a practically significant amount of IF of ${ }^{232} \mathrm{Th}$, although suppressed by a factor of 3-4 compared with ${ }^{238} \mathrm{U}$.

Nevertheless, theoretically, ${ }^{232} \mathrm{Th}$-bearing material can be actively assayed with neutron sources that produce a sufficient number of neutrons with energies above $1.5 \mathrm{MeV}$, such as AmBe (but not AmLi), PuBe, or D-D and D-T neutron generators.

While a ${ }^{252} \mathrm{Cf}$ source may also yield enough neutrons with energies above $1.5 \mathrm{MeV}$, interrogation of ${ }^{232} \mathrm{Th}$ with such a source may be generally impractical, as the signal-to-noise ratio defined as the ratio of correlated neutrons from IF of ${ }^{232} \mathrm{Th}$ to correlated neutrons from ${ }^{252} \mathrm{Cf}$ is potentially low. This assay method is referred to as the Time Correlated Induced Fission method (TCIF), which was recently developed for use of an SF source, such as ${ }^{252} \mathrm{Cf}$, in place of the traditional ${ }^{241} \mathrm{AmLi}$ source in an active detector [63]. This method has also been successfully developed for the assay of uranium enrichment in fresh LEU fuel [55]. In this and similar cases, however, the isotope of interest is ${ }^{235} \mathrm{U}$, and the low probability of fission of ${ }^{238} \mathrm{U}$ is considered an advantage of the TCIF method. Therefore, it is questionable whether practically meaningful assay of ${ }^{232} \mathrm{Th}$ using the TCIF method, where IF is further suppressed compared to ${ }^{238} \mathrm{U}$, can be performed at all.

Table 31. Relative comparison of induced fission probability of ${ }^{232}$ Th with respect to ${ }^{233} \mathrm{U},{ }^{235} \mathrm{U}$, and ${ }^{238} \mathrm{U}$ as a function of fast neutron energy (based on evaluated data from ENDF VII [49]).

\begin{tabular}{|c|c|c|c|}
\hline Neutron energy range & $\begin{array}{l}\text { Probability of IF } \\
{ }^{238} \mathrm{U} /{ }^{232} \mathrm{Th}\end{array}$ & $\begin{array}{l}\text { Probability of IF } \\
{ }^{235} \mathrm{U} /{ }^{232} \mathrm{Th}\end{array}$ & $\begin{array}{l}\text { Probability of IF } \\
{ }^{233} \mathrm{U} /{ }^{232} \mathrm{Th}\end{array}$ \\
\hline $50 \mathrm{keV}-100 \mathrm{keV}$ & 104.3 & 3135433 & 4271853 \\
\hline $100 \mathrm{keV}-1 \mathrm{MeV}$ & 64.4 & 479976 & 788852 \\
\hline $500 \mathrm{keV}-1 \mathrm{MeV}$ & 17.1 & 3316 & 5546 \\
\hline $1 \mathrm{MeV}-2 \mathrm{MeV}$ & 4.8 & 20.6 & 32.0 \\
\hline $2 \mathrm{MeV}-5 \mathrm{MeV}$ & 4.0 & 8.6 & 12.9 \\
\hline $5 \mathrm{MeV}-14 \mathrm{MeV}$ & 3.0 & 5.3 & 6.8 \\
\hline $2.45 \mathrm{MeV}$ & 5.0 & 11.5 & 17.8 \\
\hline 14.0 MeV & 3.2 & 5.8 & 6.6 \\
\hline
\end{tabular}

When considering the feasibility of active assay of ${ }^{232} \mathrm{Th}$-bearing items, however, the overall composition of the item should not be neglected. This is especially true if ${ }^{232} \mathrm{Th}$ is embedded with other fissile or fissionable material (e.g., in fresh fuel rods of Th/U-MOX types of fuel assemblies in PWR types of thorium fuel cycle). In these types of fuel, thorium oxide pellets can be homogenously or heterogeneously mixed with pellets with natural, enriched, or ${ }^{233} \mathrm{U}$-bearing pellets or fuel rods. In such cases, when actively assayed with fast neutrons (above $1.5 \mathrm{MeV}$ ), the probability of IF on all three major uranium isotopes is significantly larger than on ${ }^{232} \mathrm{Th}$ (see Table 31). Hence, if ${ }^{232} \mathrm{Th}$ is accompanied by any substantial amount of uranium during the active assay, its IF signal is not only qualitatively indistinguishable from that of any other uranium isotope, but it may be completely dwarfed by the magnitude of the IF on uranium. The ${ }^{232}$ Th fraction in such fresh fuel bundles would become effectively invisible in terms of neutron signatures. 


\section{Self-interrogation}

Self-interrogation techniques currently adopted or actively under development utilize two selfinterrogating mechanisms:

1. Neutrons emitted by the item are reflected by the item surroundings back into the item to interrogate it from the outside-in.

2. Neutrons emitted within the item interrogate the item from the inside-out.

In the first case, neutrons emitted by the item can be reflected in sufficient number only if their energies are moderated to or near thermal levels. In such cases, interrogation of ${ }^{232} \mathrm{Th}$ is entirely ineffective due to an extremely low IF cross-section. The second case can be considered only if ${ }^{232} \mathrm{Th}$ is in chemical form with other light elements, such as oxygen or fluorine. However, as ${ }^{232} \mathrm{Th}$ is a relatively weak alpha emitter (see Table 29), its $(\alpha, n)$ yield in oxide is too low $\left(2.2 \times 10^{-5} \mathrm{n} / \mathrm{g} \cdot \mathrm{s}\right)$ to be of any practical consequence, further suppressed by its own low IF cross-section. The data for $(\alpha, n)$ yields in $\mathrm{Th}(\mathrm{FLiBe})$ salt have not been independently evaluated, but even if we consider that $(\alpha, n)$ yields for beryllium and fluorine are typically $10^{3}$ and $10^{2}$ times larger than that for oxygen, respectively, it is unlikely that a sizeable sample of $\mathrm{Th}(\mathrm{FLiBe})$ salt could generate enough of any practically measurable IF neutron signal. Therefore, we have concluded that self-interrogation of ${ }^{232} \mathrm{Th}$-bearing items is not practically feasible.

\subsubsection{Uranium-233}

As discussed in Section $1,{ }^{233} \mathrm{U}$ can be found across thorium-based fuel cycles of consideration in multiple chemical and physical forms, such as ${ }^{233} \mathrm{UO}_{2},\left({ }^{233} \mathrm{U}, \mathrm{Th}\right) \mathrm{O}_{2},{ }^{233} \mathrm{UF}_{4}$, and Th(FLiBe) salt.

Passive nondestructive assay

While ${ }^{233} \mathrm{U}$ has an approximately 3.5 times greater SF yield than ${ }^{235} \mathrm{U}$ (i.e., $3.7 \times 10^{-4} \mathrm{n} / \mathrm{g}$; see Table 29), even in its purest isotopic form $\left(100 \%{ }^{233} \mathrm{U}\right)$, an item with one kilogram of ${ }^{233} \mathrm{U}$ would emit on average only about one neutron per second. ${ }^{233} \mathrm{U}$ is a strong $\alpha$-emitter and in oxide form will emit about 480 uncorrelated neutrons per 100 grams of ${ }^{233} \mathrm{U}$ content per second. That result is a sizeable and, in many potential scenarios, a statistically significant signature that can be used to verify the presence of a neutron-emitting item, but by itself is not an unambiguous signature of fissile material. The next logical question is then whether this internal $(a, n)$ neutron source inherent to ${ }^{233} \mathrm{U}$ or driver, when in a chemical form combined with a light element, can be used for self-interrogation. This concept is discussed later in this report in a section on self-interrogation.

\section{Active nondestructive assay}

${ }^{233} \mathrm{U}$ is a fissile isotope, which in terms of its fission properties is similar to ${ }^{235} \mathrm{U}$, as can be observed in Figures 60 and 61 . Table 32, which compares the IF probability between these two isotopes for various incident neutron energy ranges, shows that the probabilities of IF for both isotopes are similar and, on average, do not differ by more than a factor of two across the range of incident neutron energies, from $50 \mathrm{keV}$ to $14 \mathrm{MeV}$. In this range, ${ }^{233} \mathrm{U}$ exhibits on average an $~ 50 \%$ larger IF cross-section than ${ }^{235} \mathrm{U}$. Additionally, based on the JENDL 4.0 evaluation at a temperature of $300 \mathrm{~K}$ [38], the probability of ${ }^{233} \mathrm{U}$ fission (including the resonance region) is defined as: 


$$
\int_{E_{L}}^{E_{U}} \sigma(E, T) \cdot 1 / \mathrm{E} d E
$$

With upper and lower energy bounds $E_{L}=0.5 \mathrm{eV}$ and $E_{U}=10 \mathrm{MeV}$, it exceeds that of ${ }^{235} \mathrm{U}$ by factor of $\sim 2.8$. Conversely, however, the ratio of the two thermal fission cross-sections favors ${ }^{235} \mathrm{U}$ and is 0.91 .

Table 32. Relative comparison of induced fission probability of ${ }^{233} \mathrm{U}$ and ${ }^{235} \mathrm{U}$ as a function of incident neutron energy (based on evaluated data from ENDF VII [49]).

\begin{tabular}{|c|c|}
\hline Incident neutron energy range & $\begin{array}{l}\text { Probability of Induced Fission } \\
\qquad{ }^{233} U /{ }^{235} \mathrm{U}\end{array}$ \\
\hline $50 \mathrm{keV}-100 \mathrm{keV}$ & 1.4 \\
\hline $100 \mathrm{keV}-500 \mathrm{keV}$ & 1.6 \\
\hline $500 \mathrm{keV}-1 \mathrm{MeV}$ & 1.7 \\
\hline $1 \mathrm{MeV}-2 \mathrm{MeV}$ & 1.6 \\
\hline $2 \mathrm{MeV}-5 \mathrm{MeV}$ & 1.5 \\
\hline $5 \mathrm{MeV}-14 \mathrm{MeV}$ & 1.3 \\
\hline $2.45 \mathrm{MeV}$ & 1.5 \\
\hline $14.0 \mathrm{MeV}$ & 1.1 \\
\hline
\end{tabular}

Overall, considering the similarity of the fission properties of ${ }^{233} \mathrm{U}$ and ${ }^{235} \mathrm{U}$, it seems straightforward to extend active assay methods used for ${ }^{235} \mathrm{U}$ to ${ }^{233} \mathrm{U}$ and, therefore, to profit from the extensive body of experience with active assay of ${ }^{235} \mathrm{U}$ across all enrichment levels. Traditionally, items containing uranium-bearing material (e.g., $\mathrm{UO}_{2}$ ) are assayed in dedicated coincidence counters such as the active well coincidence counter (AWCC) [64], where an AmLi( $\alpha, n)$ neutron source is used for active interrogation [52]. A similar approach could likely be adopted for the assay of ${ }^{233} \mathrm{U}$. A similar case can be made for the extension of several other methods developed for the active assay of ${ }^{235} \mathrm{U}$, including using ${ }^{252} \mathrm{Cf}[55,56,65]$ or neutron generator techniques such as differential die-away $[65,66]$.

Therefore, it is not a question of whether an item with a known presence of ${ }^{233} \mathrm{U}$ can be assayed by active neutron NDA methods; the question is whether ${ }^{233} \mathrm{U}$ can be distinguished from ${ }^{235} \mathrm{U}$. Typically, isotopic characterization or identification is performed with complementary gamma-based measurements. However, as is discussed elsewhere in this report, such a measurement may not be trivial or at times even possible, primarily due to the heavy shielding required to protect material handlers from the presence of ${ }^{232} \mathrm{U}$, which would reduce the count rate of already low-intensity gamma rays from ${ }^{233} \mathrm{U}$ and potentially prevent their detection. Therefore, a gamma radiation measurement of shielded ${ }^{233} \mathrm{U}$, if possible, is anticipated to have a large total measurement uncertainty. Hence, the development of any neutron-based methods that would enable discrimination between these two isotopes could be of significant advantage because neutrons can penetrate shielding more easily. While, to the best of our knowledge, no such method is currently established explicitly for the assay of thorium fuel cycle materials and the discrimination of these two isotopes, it seems possible to exploit for such purposes the difference in IF probabilities as a function of energy. For example, active interrogation using fast and thermal neutron sources successively would yield results with ratios that differ significantly for ${ }^{233} \mathrm{U}$ and ${ }^{235} \mathrm{U}$. Based on the thermal fission cross-sections and data in Table 32, the fast neutron to thermal neutron interrogation yields should be $\sim 60 \%$ larger for ${ }^{233} \mathrm{U}$ than for ${ }^{235} \mathrm{U}$. Such an approach would not require explicit 
knowledge of total uranium content in the sample nor its actual geometry. With this knowledge, however, the mass of ${ }^{233} \mathrm{U}$ could be obtained. A dual-energy interrogation method has been developed for the determination of ${ }^{235} \mathrm{U}$ enrichment within uranium-bearing items [67] and could be explored for its application to the co-assay of ${ }^{233} \mathrm{U}$ and ${ }^{235} \mathrm{U}$.

\section{Self-interrogation}

In the same way that self-interrogation mechanisms were discussed in the context of ${ }^{232} \mathrm{Th},{ }^{233} \mathrm{U}$ bearing items may also be considered for self-interrogation: (1) neutrons emitted by the item are reflected into the item to interrogate it from the outside-in, or (2) neutrons emitted within the item interrogate the item from the inside-out.

Recall that, for a-emissions, the presence of light isotopes (e.g., $F, \mathrm{O}_{2}$ ) is beneficial. Given that ${ }^{233} \mathbf{U}$ is present in many instances across a variety of thorium-based nuclear fuel cycles in the form of uranium oxide $\left({ }^{233} \mathrm{UO}_{2}\right)$ or uranium fluoride $\left({ }^{233} \mathbf{U F}_{4}\right)$, and considering its significant $\alpha$-decay activity, the prospect of self-interrogation is positive, while self-interrogation can be ruled out for items from pure ${ }^{233} \mathrm{U}$ metal. Pure ${ }^{233} \mathrm{U}$ metal can potentially be assayed using active neutron interrogation instead.

In both oxide and fluoride chemical forms, the ${ }^{233} \mathrm{U}$-bearing item will emit a significant amount of $(\alpha, n)$ neutrons - 5 per gram of ${ }^{233} \mathrm{U}$ for an oxide (see Table 29) and an estimated 100 times more for a fluoride. Therefore, should the item be surrounded by a material capable of reflecting neutrons back into the item, these neutrons would be near or at thermal neutron energy levels and, hence, would have a high probability of inducing fission of ${ }^{233} \mathrm{U}$ upon reentry into the item. As the thermal fission cross-section of ${ }^{233} \mathrm{U}$ is like that of ${ }^{235} \mathrm{U}$, the infinite thickness of ${ }^{233} \mathrm{U}$ for thermal neutrons is also similar. Hence, self-interrogation by outside-in neutrons will be effective for relatively thin items such as fuel pins but may become saturated by the impact of the shape and area of the item surface if that is too bulky (i.e., if the thickness of the item is greater than the infinite thickness for thermal neutrons penetration).

Due to a significant internal $(a, n)$ source strength, every ${ }^{233} \mathrm{U}$-bearing item will also be subject to selfinterrogation from the inside-out. However, these neutrons are created with energies of $\sim 1-2 \mathrm{MeV}$ in the case of ${ }^{233} \mathrm{UF}_{4}$ and ${ }^{233} \mathrm{UO}_{2}$, respectively. Considering the limited self-moderating properties of fluoride and oxide alike, this mode of self-interrogation relies on fission induced with fast neutrons, with total probability of this interaction of approximately two to three orders of magnitude smaller than thermal fission. In other words, the probability of inducing fission by neutrons produced in $(a, n)$ reactions that never left the item to become moderated is significantly suppressed. However, without experimental evidence or detailed calculations of a specific item and assay scenario, it is not obvious whether this self-interrogation mode would result in a practically measurable signature. It is clear, however, that the prospects of self-interrogation for ${ }^{233} \mathrm{UF}_{4}$ samples are significantly enhanced compared to ${ }^{233} \mathrm{UO}_{2}$ because of its stronger internal driver (i.e., internal interrogation source) via an $\sim 100$ times stronger neutron source term.

From an alternative perspective, we can consider self-interrogation assay of LEU in the form of lowenriched $\mathrm{UF}_{6}$ within large LEU cylinders such as Type 30B and Type 48Y [60]. In the case of large LEU cylinders, the neutron source term is mostly driven by a-decay of ${ }^{234} \mathrm{U}$, with typical concentrations of ${ }^{234} \mathrm{U}$ of $5 \times 10^{-5}$ to $5 \times 10^{-4}$ by weight, and ${ }^{235} \mathrm{U}(0.2-5 \%$ enrichment) being the IF target. Because the $(\alpha, n)$ 
yield of ${ }^{233} \mathrm{U}$ and ${ }^{234} \mathrm{U}$ is very similar $\left(3.5 \times 10^{8} \mathrm{vs} .2 .3 \times 10^{8} \mathrm{a} / \mathrm{g} \cdot \mathrm{s}\right)$, as is the IF cross-section of ${ }^{233} \mathrm{U}$ and ${ }^{235} \mathrm{U}$, we have only to consider the relative concentrations and amount of LEU in a Type 30B cylinder. This type of container typically holds $\sim 2000 \mathrm{~kg}$ of uranium, and its self-interrogating signature is strong enough for practical safeguards purposes. Items containing ${ }^{233} \mathrm{UF}_{4}$ have an approximately $2 \times 10^{3}$ to $2 \times 10^{4}$ times larger concentration of the $(\alpha, n)$ initiating isotope ${ }^{233} U$ when compared to the concentration of ${ }^{234} \mathrm{U}$ in a Type 30B container. Therefore, it could be inferred that an item with $\sim 100 \mathrm{~g}$ of ${ }^{233} \mathrm{U}$ in the form of ${ }^{233} \mathrm{UF}_{4}$ could yield a self-interrogating signature of similar magnitude to a Type $30 \mathrm{~B}$ cylinder filled with $5 \%$ enriched LEU. Should that be the case, such an item is assayable via selfinterrogation with already-existing instrumentation dedicated for coincidence or multiplicity counting of similarly sized uranium or plutonium samples from conventional U/Pu nuclear fuel cycles.

Additionally, comparison of passively obtained self-interrogation results with results of active assay could enable discrimination between ${ }^{235} \mathrm{U}$ and ${ }^{233} \mathrm{U}$ in the case of inconclusive or absent complementary gamma measurements, as self-interrogation of small items with ${ }^{235} \mathrm{U}(\sim 100$ grams $)$ is not practically possible due to the low $\alpha$-decay rate of ${ }^{235} \mathrm{U}$.

\subsubsection{Overview of Simulated and Experimental Validation of Selected Neutron Signature Properties}

One of the main objectives of this scoping study was to investigate signatures of nuclear material relevant to thorium fuel cycles that could be used for nuclear material assay under international nuclear safeguards. In the previous section, based on fundamental physics properties of the most prominent thorium fuel cycle materials, the case has been made for the potential feasibility (or lack thereof) of using different NDA modes for the measurement of these materials. The next step in this study was to perform simulated and experimental validation of the theoretical assumptions and conclusions. However, only selected experiments were performed based on the limited availability of and access to relevant nuclear material.

In the case of U/Pu nuclear material accountancy, R\&D capabilities have been long established and profited from several generations of safeguards practitioners and researchers supported by established infrastructure that includes the availability of certified reference material, in addition to mock-up fuel cycle items and calibration standards. In the case of thorium fuel cycle-relevant material, no easily available certified NDA sources currently exist, preventing instrument calibration or sensitivity validation based on reference values. However, a limited range of items were made available for this study, with the caveat that they resembled a set of ad hoc items fabricated without prior regard for their use with a suite of NDA equipment typical of U/Pu NDA methods. The half-dozen thorium-bearing items contained masses from $\sim 15 \mathrm{~g}$ to $3 \mathrm{~kg}$ of thorium in forms of pure metal, oxide, and carbide. ${ }^{233} \mathrm{U}$-bearing items varied only marginally in mass, from $\sim 1 \mathrm{~g}$ to $10 \mathrm{~g}$, with contamination of only a few ppm of ${ }^{232} \mathrm{U}$. These ${ }^{233} \mathrm{U}$ samples, however, still required bulky lead-shielding containers of dimensions that did not fit into any of the neutron well counters utilized in this study. Moreover, NDA of bare ${ }^{233} \mathrm{U}$ items would have been possible only in a shielded cell due to the presence of ${ }^{232} \mathrm{U}$ progeny, which was an activity not undertaken during this project. As a result, selected measurements should not be considered as comprehensive verification of conclusions based on fundamental physics principles, but instead may serve as a template for any future dedicated efforts that combine theoretical models and discrete measurements of simple yet representative items enabling 
benchmarking of high-fidelity simulations. Those, in turn, can be used for extrapolation into scenarios that cannot be readily staged in laboratory conditions outside nuclear facilities.

The following sections provide an evaluation of the capabilities of two neutron coincidence counters. Both counters are currently used by the IAEA for international nuclear safeguards applications. The AWCC has been selected as a well-benchmarked neutron coincidence counter used for active interrogation of uranium-bearing items, while the epithermal neutron multiplicity counter (ENMC) is an example of a high-efficiency detector used for passive neutron multiplicity counting of plutoniumbearing items. Both the AWCC and ENMC will be explored for their applicability to thorium- and ${ }^{233} \mathrm{U}$ bearing nuclear material.

High-fidelity MCNP6 models of both detectors were first validated with experimental and analytical data, adjusting the models, if needed. MCNP simulations were then conducted of the active (AWCC) and passive (ENMC) assay of several thorium-bearing items. Finally, the simulated performance of both detectors on composite items discussed in Sections 1 and 2 were evaluated. These composite items were chosen for their representation of specific thorium fuel cycles in this study.

\subsubsection{ENMC and AWCC Model Validation}

MCNP models of the ENMC (Figure 71) [68] and of the AWCC (Figure 72) [64] were first validated against analytical and experimental data. Several sources were used: $\mathrm{PuO}_{2}$ STD 1, $\mathrm{PuO}_{2}$ STD 2, ${ }^{252} \mathrm{Cf}$ Source 1 , ${ }^{252} \mathrm{Cf}$ Source 2, and AmLi Source 1 . This section begins with the plutonium oxide sources, which are described in Table 33.
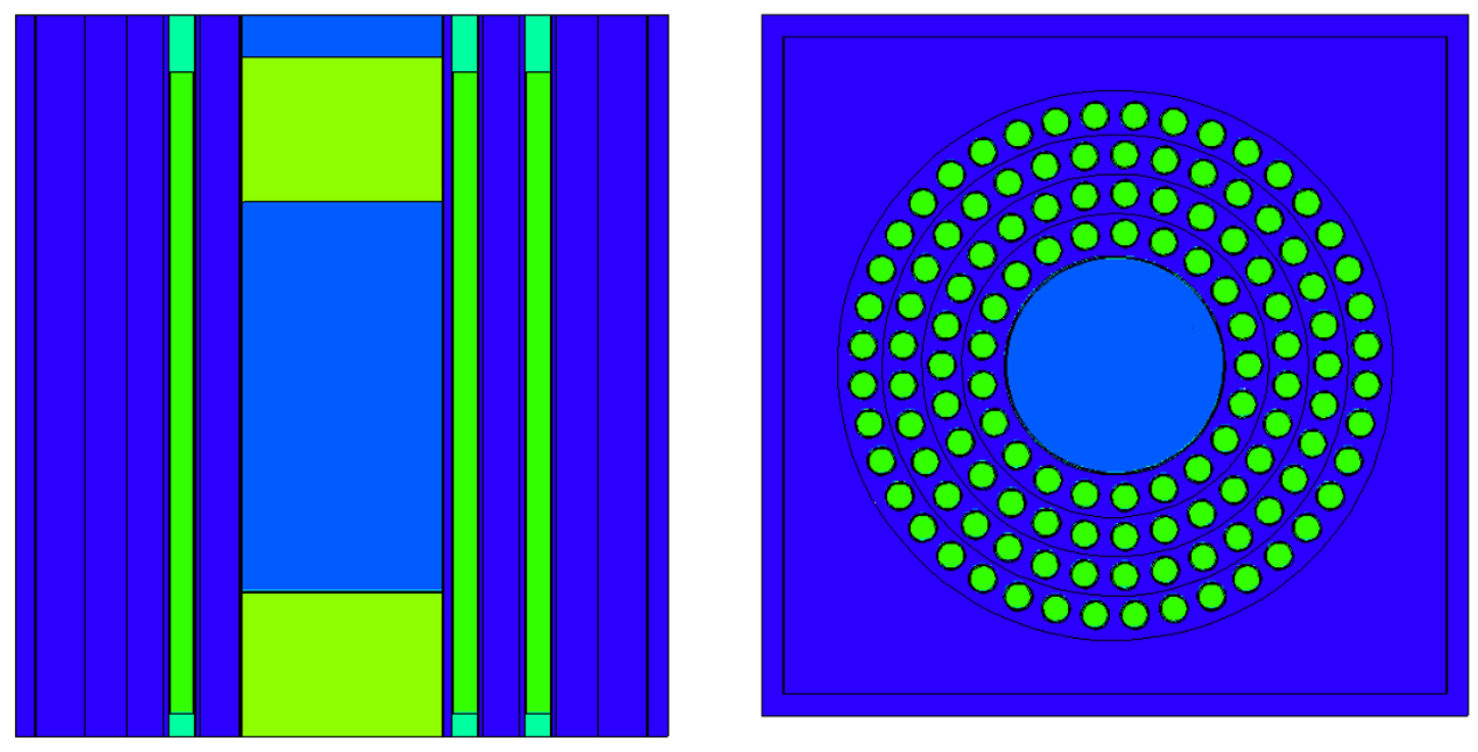

Figure 71. ENMC vertical (left) and horizontal (right) views. 

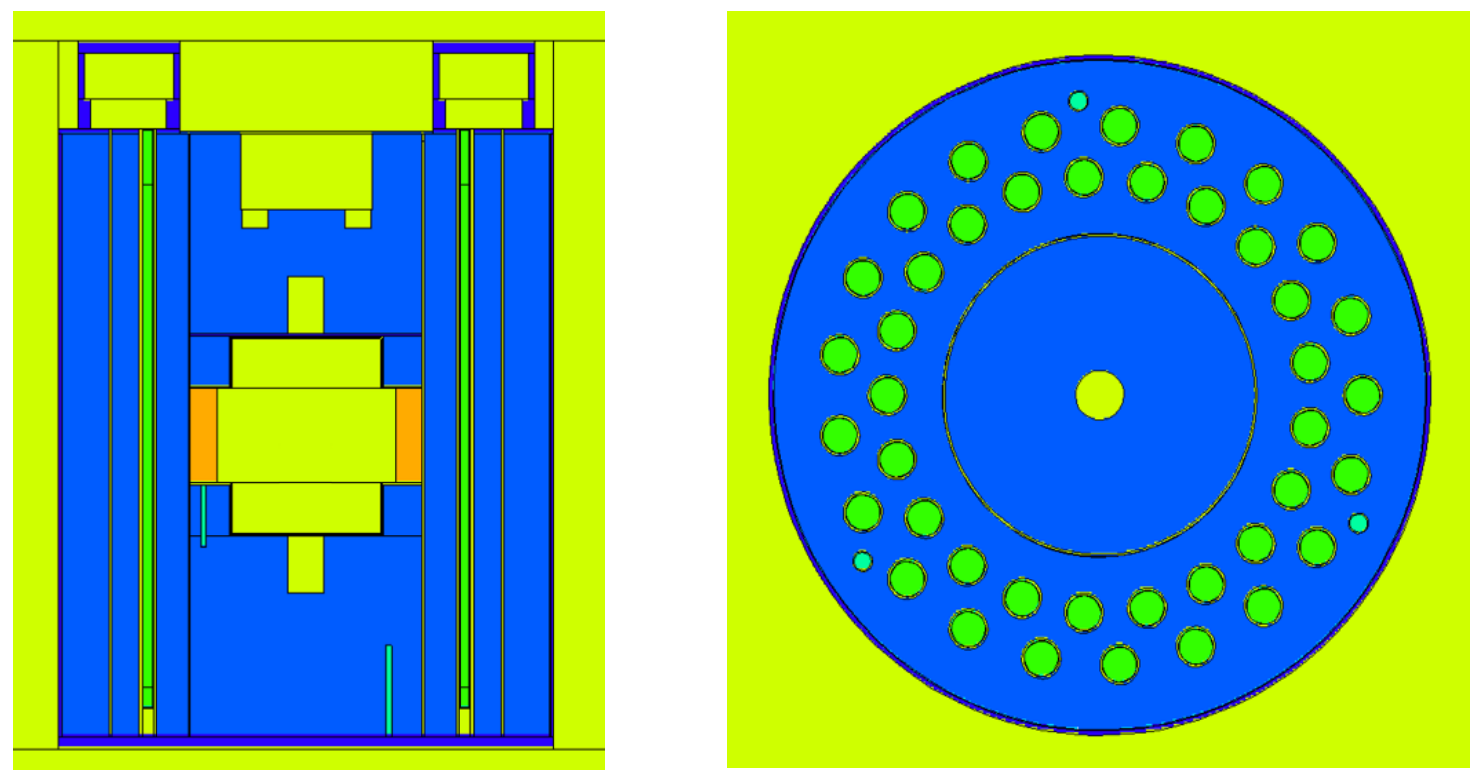

Figure 72. AWCC vertical (left) and horizontal (right) views.

Table 33. Main characteristics of the plutonium sources used for ENMC and AWCC model validation. The plutonium isotope mass fractions are normalized to 100 , and the ${ }^{241} \mathrm{Am}$ fraction is referred to the plutonium content.

\begin{tabular}{|c|c|c|c|c|c|c|c|c|}
\hline Source ID & Material & Mass & ${ }^{238} \mathrm{Pu}$ & ${ }^{239} \mathrm{Pu}$ & ${ }^{240} \mathrm{Pu}$ & ${ }^{241} \mathrm{Pu}$ & ${ }^{242} \mathrm{Pu}$ & ${ }^{241} \mathrm{Am}$ \\
\hline $\mathrm{PuO}_{2} \mathrm{STD} 1$ & $\mathrm{PuO}_{2}$ & $30 \mathrm{~g}$ & 0.0063 & 96.32 & 3.562 & 0.094 & 0.018 & 0.343 \\
\hline $\mathrm{PuO}_{2}$ STD 2 & $\mathrm{PuO}_{2}$ & $50 \mathrm{~g}$ & 0.0063 & 96.32 & 3.562 & 0.094 & 0.018 & 0.343 \\
\hline
\end{tabular}

Coincidence counting is simulated in the MCNP model by means of the following cards within the input deck:

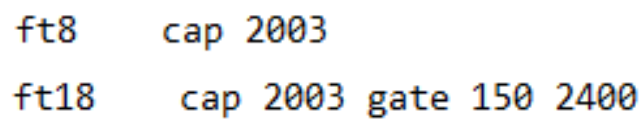

The first line simulates an ungated tally, which scores the neutron singles count rate, and the second line simulates a tally with coincidence counting parameters of a $1.5 \mu$ s predelay and a $24 \mu \mathrm{s}$ coincidence timing gate, which scores the coincidence count rates. Two simulations were carried out for each source, one to count coincidences due to neutrons from SF and another to evaluate the $(a, n)$ contribution from a-particle capture on oxygen. The neutron counts for the SF simulation of $\mathrm{PuO}_{2}$ STD 1 are extracted from the output file and given below. 

factorial moments
by number
by weight
3he
$1.51126 \mathrm{E}+00 \quad 0.0025$
$7.02653 \mathrm{E}-010.0025 \longrightarrow$ SINGLES
factorial moments
by number
by weight

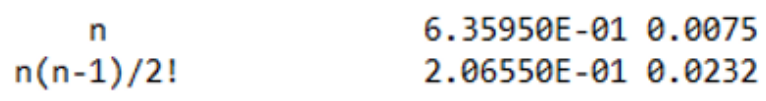

A similar output was also obtained for the $(a, n)$ contribution. Because the counts are normalized by the number of source particles used in the simulation, they need to be multiplied by the SF and the $(a, n)$ intensities of the real source to obtain the count rates. The intensities,

$I_{a n}$ for $(\alpha, \mathrm{n})$ and $I_{s f}$ for SF, can be calculated as follows:

$$
\begin{gathered}
I_{s f}=m G v_{s} \\
I_{a n}=\alpha m G v_{s}
\end{gathered}
$$

where $m$ is the equivalent ${ }^{240} \mathrm{Pu}_{\text {eff }}$ mass:

$$
m=\left(2.52 \text { Pu } 238+\text { Pu } 240+1.68 \text { Pu240) } \cdot \frac{\text { source mass }(g)}{100}\right.
$$

$G$ is the ${ }^{240} \mathrm{Pu}$ SF yield (473 fiss $/ \mathrm{sg}$ ), $v_{s}$ is the SF multiplicity (2.16 [1]), and the quantity $\alpha$ is the $(\alpha, \mathrm{n})$ to SF yield ratio:

$$
\alpha=\frac{13400 f_{\text {Pu } 238}+38.1 f_{\text {Pu } 239}+141 f_{\text {Pu } 240}+1.3 f_{\text {Pu } 241}+2.0 f_{\text {Pu242 }}+2690 f_{\text {Am } 241}}{\left(2.52 f_{\text {Pu } 238}+f_{\text {Pu } 240}+1.68 f_{\text {Pu } 242}\right) G v_{S}}
$$

Using the tally results and Equations 1-4, singles, doubles, and triples neutron count rates are derived for $\mathrm{PuO}_{2}$ STD 1 and $\mathrm{PuO}_{2}$ STD 2 (Tables 34 and 35).

Table 34. Simulated singles, doubles, and triples for $\mathrm{PuO}_{2}$ STD 1 (ENMC).

\begin{tabular}{|l|c|c|c|}
\hline \multirow{2}{*}{ Spontaneous fission } & $\begin{array}{c}\text { Singles (counts/s) } \\
\text { (\% relative error) }\end{array}$ & $\begin{array}{c}\text { Doubles (counts/s) } \\
\text { (\% relative error) }\end{array}$ & $\begin{array}{c}\text { Triples (counts/s) } \\
\text { (\% relative error) }\end{array}$ \\
& 775.60 & 328.69 & 108.67 \\
& $(0.02)$ & $(0.08)$ & $(0.25)$ \\
\hline$(a, n)$ & 1058.46 & 66.06 & 28.43 \\
& $(0.03)$ & $(0.36)$ & $(0.90)$ \\
\hline Total & 1834.06 & 394.75 & 137.10 \\
& $(0.03)$ & $(0.13)$ & $(0.38)$ \\
\hline
\end{tabular}


Table 35. Simulated singles, doubles, and triples for $\mathrm{PuO}_{2}$ STD 2 (ENMC).

\begin{tabular}{|l|c|c|c|}
\multicolumn{2}{|c|}{$\begin{array}{c}\text { Singles (counts/s) } \\
\text { (\% relative error) }\end{array}$} & $\begin{array}{c}\text { Doubles (counts/s) } \\
\text { (\% relative error) }\end{array}$ & $\begin{array}{c}\text { Triples (counts/s) } \\
\text { (\% relative error) }\end{array}$ \\
\hline Spontaneous fission & 1309.02 & 574.50 & 205.73 \\
& $(0.02)$ & $(0.07)$ & $(0.26)$ \\
\hline$(\alpha, \mathrm{n})$ & 1789.20 & 132.42 & 61.08 \\
& $(0.03)$ & $(0.34)$ & $(1.03)$ \\
\hline Total & 3098.22 & 706.92 & 266.81 \\
& $(0.03)$ & $(0.12)$ & $(0.44)$ \\
\hline
\end{tabular}

\subsubsection{MCNP Model Validation: Analytical Comparison to Point model}

The point model [69] is employed to verify MCNP singles, doubles, and triples tally count rate results, which were provided in Tables 34 and 35. According to this model, the singles and doubles neutron count rates due to SFs and $(\alpha, n)$ reactions can be derived as follows:

Spontaneous fission singles:

$(a, n)$ singles:

$$
S_{s f}=m G e_{s f} M_{s f} v_{s}
$$

$$
S_{a n}=\alpha m G v_{s} M_{a n} e_{a n}
$$

Spontaneous fission doubles:

$$
D_{s f}=m G \varepsilon_{s f}^{2} F_{d, s f} M_{s f}^{2}\left[\overline{\frac{v_{s}\left(v_{s}-1\right)}{2}}+\left(M_{s f}-1\right) \frac{\overline{v_{s}} \overline{v_{l}\left(v_{l}-1\right)}}{2\left(\overline{v_{i}}-1\right)}\right]
$$

$(a, n)$ doubles:

$$
D_{a n}=\alpha m G v_{s} \varepsilon_{a n}^{2} F_{d, a n} M_{a n}^{2}\left[\left(M_{a n}-1\right) \frac{\overline{v_{l}\left(v_{l}-1\right)}}{2\left(\overline{v_{i}}-1\right)}\right]
$$

where the following quantities need to be calculated using the MCNP model:

$M_{s f}=$ spontaneous fission net multiplication

$M_{\text {an }}=(\alpha, n)$ net multiplication

$e_{s f}=\frac{(F 4 \text { tally total bin })_{s f}}{M_{s f}}$

$e_{a n}=\frac{(F 4 \text { tally total bin })_{a n}}{M_{a n}}$

$v_{s}=$ First moment of spontaneous fission neutron multiplicity

$\frac{\frac{v_{s}\left(v_{s}-1\right)}{2}}{2}=$ Second moment of spontaneous fission neutron multiplicity

$v_{i}=$ First moment of induced fission neutron multiplicity

$\frac{\overline{v_{l}\left(v_{l}-1\right)}}{2}=$ Second moment of induced fission neutron multiplicity

$F_{d, s f}=\frac{(F 4 \text { tally } 1.5 \mu s-24 \mu \text { s time bin })_{s f}}{(F 4 \text { tally total time bin })_{s f}}=\frac{0.4249}{0.7037}=$ Spontaneous fission double gate fraction 


$$
F_{d, a n}=\frac{(F 4 \text { tally } 1.5 \mu s-24 \mu \text { s time bin })_{a n}}{(F 4 \text { tally total time bin })_{a n}}=\frac{0.4050}{0.6799}=(\alpha, n) \text { double gate fraction }
$$

From Equations 5-8, the results shown in Tables 36 and 37 were obtained.

Table 36. Calculated singles and doubles for $\mathrm{PuO}_{2}$ STD 1 (ENMC).

\begin{tabular}{|c|c|c|}
\hline & Singles (counts/s) & Doubles (counts/s) \\
\hline Spontaneous fission & 774.64 & 334.72 \\
\hline$(a, n)$ & 1058.02 & 61.21 \\
\hline Total & 1832.66 & 395.93 \\
\hline
\end{tabular}

Table 37. Calculated singles and doubles for $\mathrm{PuO}_{2}$ STD 2 (ENMC).

Singles (counts/s) Doubles (counts/s)

\begin{tabular}{|l|l|l|}
\hline Spontaneous fission & 1307.88 & 585.55 \\
\hline$(a, n)$ & 1778.84 & 128.57 \\
\hline Total & 3086.72 & 714.12 \\
\hline
\end{tabular}

\subsubsection{Comparison with Experiment and Discussion of Results}

Tables 38 and 39 provide a comparison of the total singles, doubles, and triples neutron count rates simulated by MCNP and evaluated using the point model to verify experimental data. Where the neutron count rates are left blank within these results tables and others below, this is an indication of very low count rates.

The MCNP simulations are in good agreement with the point model results, while experimental data show significant differences. Further investigation showed that the discrepancies resulted from the mixture of the $\mathrm{PuO}_{2}$ powder of $\mathrm{PuO}_{2}$ STD 1 and $\mathrm{PuO}_{2}$ STD 2 with a matrix of diatomaceous earth. The resulting impurities (e.g., fluorine) contained in the sources strongly increased the $(a, n)$ neutron intensity. In light of this, Equation 4 is no longer suitable to calculate $\alpha$. In more detail, using the multiplicity verification approach within the IAEA Neutron Coincidence Counting (INCC) code [70], it was estimated that (a) $\alpha$ is equal to 4.586 for $\mathrm{PuO}_{2}$ STD 1 and 4.372 for $\mathrm{PuO}_{2}$ STD 2, and (b) multiplication $M$ is equal to 1 for both sources because the powder is dispersed in the matrix, thus reducing its "effective" density. The two sources were, in fact, examples of sources used for the simulation of nuclear waste. Accounting for this information and renormalizing the MCNP results accordingly, agreement was obtained with the experimental data. The corrected MCNP results are also included in Table 38, where the corrected values are based on the alpha values. 
Table 38. Calculated vs. simulated vs. measured count rates for $\mathrm{PuO}_{2}$ STD 1 (ENMC).

\begin{tabular}{|c|c|c|c|c|c|c|}
\hline & $\begin{array}{l}\text { Point } \\
\text { model }\end{array}$ & $\begin{array}{c}\text { MCNP } \\
(\% \\
\text { Relative } \\
\text { error })\end{array}$ & $\begin{array}{l}\text { Percent difference } \\
\text { between Point } \\
\text { model and MCNP }\end{array}$ & $\begin{array}{c}\text { MCNP } \\
\text { corrected } \\
\text { (\% } \\
\text { Relative } \\
\text { error) }\end{array}$ & Experimental & $\begin{array}{l}\text { Percent difference } \\
\text { between MCNP } \\
\text { corrected and } \\
\text { experimental }\end{array}$ \\
\hline $\begin{array}{l}\text { Single } \\
\text { (counts/s) }\end{array}$ & 1832.66 & $\begin{array}{c}1834.06 \\
(0.03)\end{array}$ & 0.08 & $\begin{array}{c}3891.81 \\
(0.03)\end{array}$ & 3875.44 & -0.42 \\
\hline $\begin{array}{l}\text { Double } \\
\text { (counts/s) }\end{array}$ & 395.93 & $\begin{array}{c}394.75 \\
(0.13)\end{array}$ & -0.30 & $\begin{array}{c}250.39 \\
(0.08)\end{array}$ & 242.69 & -3.08 \\
\hline \begin{tabular}{|l|} 
Triples \\
(counts/s)
\end{tabular} & - & $\begin{array}{l}137.10 \\
(0.38)\end{array}$ & - & $\begin{array}{l}46.84 \\
(0.26)\end{array}$ & 46.22 & -1.32 \\
\hline
\end{tabular}

Table 39. Calculated vs. simulated vs. measured count rates for $\mathrm{PuO}_{2}$ STD 2 (ENMC).

\begin{tabular}{|c|c|c|c|c|c|c|}
\hline & $\begin{array}{l}\text { Point } \\
\text { model }\end{array}$ & $\begin{array}{l}\text { MCNP } \\
(\% \\
\text { Relative } \\
\text { error) }\end{array}$ & $\begin{array}{l}\text { Percent difference } \\
\text { between Point } \\
\text { model and MCNP }\end{array}$ & $\begin{array}{c}\text { MCNP } \\
\text { corrected } \\
\text { (\% } \\
\text { Relative } \\
\text { error) }\end{array}$ & Experimental & $\begin{array}{l}\text { Percent difference } \\
\text { between MCNP } \\
\text { corrected and } \\
\text { experimental }\end{array}$ \\
\hline $\begin{array}{l}\text { Single } \\
\text { (counts/s) }\end{array}$ & 3086.72 & $\begin{array}{c}3098.22 \\
(0.03)\end{array}$ & 0.37 & $\begin{array}{c}6248.58 \\
(0.03)\end{array}$ & 6227.22 & -0.34 \\
\hline $\begin{array}{l}\text { Double } \\
\text { (counts/s) }\end{array}$ & 714.12 & $\begin{array}{c}706.92 \\
(0.12)\end{array}$ & -1.01 & $\begin{array}{c}412.31 \\
(0.07)\end{array}$ & 408.11 & -1.02 \\
\hline $\begin{array}{l}\text { Triples } \\
\text { (counts/s) }\end{array}$ & - & $\begin{array}{c}266.81 \\
(0.44)\end{array}$ & - & $\begin{array}{l}75.51 \\
(0.26)\end{array}$ & 78.32 & 3.72 \\
\hline
\end{tabular}

\subsubsection{3 ${ }^{252} \mathrm{Cf}$ and AmLi Sources for Active Neutron NDA}

Further empirical validation of the MCNP model of the ENMC, described earlier in this section, was carried out using two ${ }^{252} \mathrm{Cf}$ sources (i.e., Cf Source 1 and $\mathrm{Cf}$ Source 2 ) and an AmLi source (i.e., AmLi Source 1). For the source simulations, the ${ }^{252} \mathrm{Cf}$ source neutron energy distribution was modeled as the Watt fission spectrum embedded in MCNP, while the neutron production from the AmLi source was modeled using the Obnisk neutron spectrum, available at Los Alamos National Laboratory (LANL) [71]. The source intensities from the decay-corrected source certificate values are listed in Table 40, while comparisons between MCNP simulations and experiments are presented in Tables 41-43. Agreement is not fully satisfactory for all measurements, with errors ranging from $1.5 \%$ to $26 \%$.

Table 40. ${ }^{252} \mathrm{Cf}$ and AmLi source intensities from the decay-corrected source certificate values.

\begin{tabular}{|c|c|}
\hline Source ID & Intensity (neutrons/s) \\
\hline Cf Source 1 & 181.81 \\
\hline Cf Source 2 & 362979.47 \\
\hline AmLi Source 1 & 51500.00 \\
\hline
\end{tabular}


Table 41. Calculated vs. measured count rates for Cf Source 1 (ENMC).

\begin{tabular}{|c|c|c|c|}
\hline $\begin{array}{c}\text { Source ID: } \\
\text { Cf Source 1 }\end{array}$ & $\begin{array}{c}\text { MCNP } \\
\text { (\% Relative error) }\end{array}$ & \multicolumn{3}{c|}{ Experiment Percent Difference } \\
\hline Single (counts/s) & $\begin{array}{c}117.35 \\
(0.16)\end{array}$ & 159 & $-26.19 \%$ \\
\hline Double (counts/s) & $\begin{array}{l}71.23 \\
(0.51)\end{array}$ & 77 & $-7.49 \%$ \\
\hline Triples (counts/s) & $\begin{array}{l}24.86 \\
(0.95)\end{array}$ & 26 & $-4.38 \%$ \\
\hline
\end{tabular}

Table 42. Calculated vs. measured count rates for Cf Source 2 (ENMC).

\begin{tabular}{|c|c|c|c|}
\hline \multicolumn{1}{|c|}{$\begin{array}{c}\text { Source ID: } \\
\text { Cf Source 2 }\end{array}$} & $\begin{array}{c}\text { MCNP } \\
\text { (\% Relative error) }\end{array}$ & Experiment & Percent Difference \\
\hline Single (counts/s) & $\begin{array}{c}234403.33 \\
(0.16)\end{array}$ & 228992.71 & $2.36 \%$ \\
\hline Double (counts/s) & $\begin{array}{c}142282.72 \\
(0.51)\end{array}$ & 138867.71 & $2.46 \%$ \\
\hline Triples (counts/s) & $\begin{array}{c}49635.13 \\
(0.95)\end{array}$ & 44308.91 & $12.02 \%$ \\
\hline
\end{tabular}

Table 43. Calculated vs. measured count rates for AmLi Source 1 (ENMC).

\begin{tabular}{|c|c|c|c|}
\hline $\begin{array}{c}\text { Source ID: } \\
\text { AmLi Source 1 }\end{array}$ & $\begin{array}{c}\text { MCNP } \\
\text { (\% Relative error) }\end{array}$ & Experiment Percent Difference \\
\hline Single (counts/s) & $\begin{array}{c}37552.77 \\
(0.19)\end{array}$ & 30291 & $23.97 \%$ \\
\hline Double (counts/s) & - & 1 & $-100.00 \%$ \\
\hline Triples (counts/s) & - & - & - \\
\hline
\end{tabular}

\subsubsection{AWCC Model Validation: AmLi Source}

The AmLi Source 1 was also used to verify the AWCC model. Table 44 lists the single count rates in all the ${ }^{3} \mathrm{He}$ counters in both the inner and outer detector rings. Good agreement is obtained with experimental data, with errors consistently below $5 \%$. Double and triple neutron count rates are negligible in both the simulation and in the experiment, as expected from a noncorrelated AmLi neutron source. 
Table 44. Calculated vs. measured count rates for AmLi Source 1 (AWCC).

\begin{tabular}{|l|c|c|c|}
\multicolumn{1}{|c|}{$\begin{array}{c}\text { Source ID: } \\
\text { AmLi Source 1 }\end{array}$} & $\begin{array}{c}\text { MCNP } \\
\text { (\% Relative error) }\end{array}$ & Experiment & Percent Difference \\
\hline $\begin{array}{l}\text { Single (counts/s) } \\
\text { All }{ }^{3} \text { He counters }\end{array}$ & $\begin{array}{c}8414.07 \\
(0.72)\end{array}$ & 8414 & $-0 \%$ \\
\hline Single (counts/s) & $\begin{array}{c}5882.55 \\
(0.88)\end{array}$ & 5745 & $2.39 \%$ \\
Inner ring & $\begin{array}{c}2531.74 \\
(1.39)\end{array}$ & 2663 & $-4.82 \%$ \\
\hline Single (counts/s) & & \\
\hline Outer ring & & & \\
\hline
\end{tabular}

\subsubsection{MCNP Simulations: Passive and Active Assay of Thorium-bearing Material}

Having validated the ENMC and AWCC models, the use of these two detection systems in novel ways (i.e., for the assay of thorium and ${ }^{233} \mathrm{U}$ ) can be explored. This section reports the results of passive and active assay of thorium samples. The characteristics of the considered thorium sources are listed in Table 45.

Table 45. Characteristics of the considered thorium sources.

\begin{tabular}{|c|c|c|}
\hline Source ID & Material & Thorium mass (g) \\
\hline Th Source 1 & Metal thorium & 15 \\
\hline Th Source 2 & Metal thorium & 31.5 \\
\hline Th Source 3 & $\mathrm{ThO}_{2}$ & 68 \\
\hline Th Source 4 & Metal thorium & 3000 \\
\hline
\end{tabular}

\subsubsection{Passive Assay of Thorium}

Due to the low SF neutron yield of thorium, passive assay is not expected to be suitable for thorium measurements. Therefore, two sources were simulated using the ENMC model to validate the following conclusions:

- Th Source 3 was simulated to assess whether $(a, n)$ neutrons give a significant contribution to the low passive neutron count rates.

- Th Source 4 was simulated to evaluate whether SF leads to measurable count rates at kilogram quantities.

Single neutron count rates were on the order of $10^{-3}$ counts/s for Th Source 3 and $10^{-4}$ counts $/ \mathrm{s}$ for Th Source 4, while coincidence count rates were not scored at all for both sources due to their low SF neutron yields. The measurable differences in the singles neutron count rates is due to the small contribution from $(a, n)$ neutrons in the Th Source 3 oxide. These results are also in agreement with conducted experiments. 
Table 46. Results for passive measurements performed using ENMC.

\begin{tabular}{|c|c|c|c|c|c|c|c|}
\hline Source ID & \multicolumn{2}{c}{${ }^{232}$ Th mass (g) } & \multicolumn{2}{c|}{ Singles Singles Sigma } & Doubles & Doubles Sigma & Triples Triples Sigma \\
\hline Th Source 3 & 3000 & 1.683 & 0.581 & 1.668 & 0.885 & 3.285 & 2.233 \\
\hline Th Source 4 & 15 & 0.292 & 0.481 & 0.527 & 0.178 & 0.677 & 0.330 \\
\hline
\end{tabular}

\subsubsection{Active Assay Active Assay of Thorium}

Active thorium assay was simulated with the AWCC model, considering all the sources listed in Table 45. To induce fissions in the samples, the AmLi Source 1 was placed in the upper plug of the detector (see Figure 73).

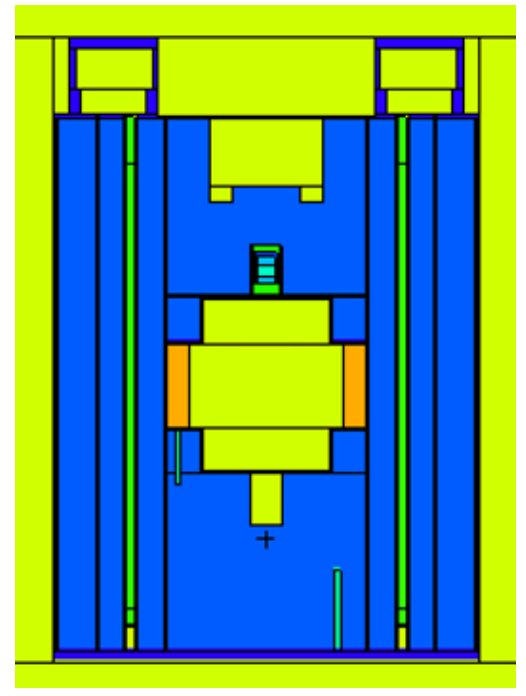

Figure 73. AWCC model with the AmLi source (green).

From simulations, small differences in the singles count rates were observed, compared to the case in which no source was placed into the detector (Table 47). Coincidence count rates (doubles and triples) were negligible in all the simulated cases because the fission cross-section of thorium was small for neutron energies typical of the AmLi source, and IFs did not add a significant contribution to AmLi-emitted neutrons. In fact, virtually all the scored neutrons were emitted by AmLi Source 1, except when kilogram quantities of thorium were assayed.

Table 47. Single count rates for thorium samples interrogated by AmLi Source 1.

\begin{tabular}{|l|c|}
\hline Source ID & $\begin{array}{l}\text { Singles (counts/s) } \\
\text { (\% relative error) }\end{array}$ \\
\hline No sample & $2354.58(0.14)$ \\
\hline Th Source 1 & $2355.76(0.14)$ \\
\hline Th Source 2 & $2356.90(0.14)$ \\
\hline Th Source 3 & $2362.82(0.14)$ \\
\hline Th Source 4 & $2470.97(0.14)$ \\
\hline
\end{tabular}


As a potential way to overcome this limitation, ${ }^{252} \mathrm{Cf}$ may be used in place of $\mathrm{AmLi}$ interrogation sources, noting that the higher energy of SF neutrons compared to $\operatorname{AmLi}(\alpha, n)$ may induce fast fissions in thorium. The suitability of this option was assessed by replacing AmLi Source 1 with Cf Source 2 . Compared to the AmLi case, doubles became measurable (see Table 48). However, there was little difference between measurements carried out with or without the sample (except when kilogram quantities of ${ }^{232} \mathrm{Th}$ were assayed). This points out that the majority of coincidences were due to SFs occurring in ${ }^{252} \mathrm{Cf}$, rather than to IFs in ${ }^{232} \mathrm{Th}$. These results suggest that active coincidence counting may not be a good option for the assay of pure ${ }^{232} \mathrm{Th}$, when the source used is AmLi or ${ }^{252} \mathrm{Cf}$. Sources such as AmBe or D-D and D-T should be evaluated in the future as alternative sources because they produce neutrons with higher energy (see Figure 12) that can achieve greater IF of ${ }^{232} \mathrm{Th}$.

Table 48. Single count rates for thorium samples (AmLi Source 1).

\begin{tabular}{|c|c|c|}
\hline Source ID & $\begin{array}{l}\text { Singles (counts/s) } \\
\text { (\% relative error) }\end{array}$ & $\begin{array}{l}\text { Doubles (counts/s) } \\
\text { (\% relative error) }\end{array}$ \\
\hline No sample & $36207.15(0.07)$ & $3847.57(0.26)$ \\
\hline Th Source 1 & $36218.04(0.07)$ & $3858.46(0.26)$ \\
\hline Th Source 2 & $36225.30(0.07)$ & $3861.01(0.26)$ \\
\hline Th Source 3 & $36261.60(0.07)$ & $3869.36(0.26)$ \\
\hline Th Source 4 & $37023.86(0.07)$ & $4050.85(0.25)$ \\
\hline
\end{tabular}

\subsubsection{MCNP Simulations: Active Assay of Nixed ${ }^{233} \mathrm{U} /{ }^{232}$ Th Samples in AWCC}

Pure thorium sources lead to impractically weak signals both in active and passive coincidence counters. This is because thorium is a fertile nuclide, not fissile, and its SF probability is low. Nevertheless, active coincidence counting in the AWCC may be useful for the assay of mixed ${ }^{233} \mathrm{U} /{ }^{232} \mathrm{Th}$ samples due to the high neutron-induced fission cross-section of ${ }^{233} \mathrm{U}$.

To this purpose, Th Source 4 was modified in the model to include a ${ }^{233} \mathrm{U}$ fraction, from $1 \%$ to $4 \%$ of mass. As shown by Table 49, there was a slight but measurable increase of both singles and doubles neutron count rates as the ${ }^{233} \mathrm{U}$ mass fraction increased. The doubles count rate as a function of the ${ }^{233} \mathrm{U}$ mass is also presented in Figure 74 . The significant increase of doubles for increasing ${ }^{233} \mathrm{U}$ mass fractions suggests that active assay techniques can be applied to the measurement of ${ }^{233} \mathrm{U}$ content (or other fissile isotopes) in thorium-based fuels. 
Table 49. Singles and doubles count rates for a ${ }^{233} \mathrm{U}$-enriched/thorium-bearing sample.

\begin{tabular}{|l|c|c|}
\hline${ }^{233}$ U mass enrichment (\%) & $\begin{array}{c}\text { Singles (counts/s) } \\
\text { (\% relative error) }\end{array}$ & $\begin{array}{c}\text { Doubles (counts/s) } \\
\text { (\% relative error) }\end{array}$ \\
\hline 0 & $2470.97(0.14)$ & $\sim 0$ \\
\hline 1 & $2536.06(0.14)$ & $16.74(2.3)$ \\
\hline 2 & $2581.03(0.14)$ & $28.68(1.8)$ \\
\hline 3 & $2615.60(0.14)$ & $37.79(1.6)$ \\
\hline 4 & $2646.03(0.14)$ & $45.17(1.4)$ \\
\hline
\end{tabular}

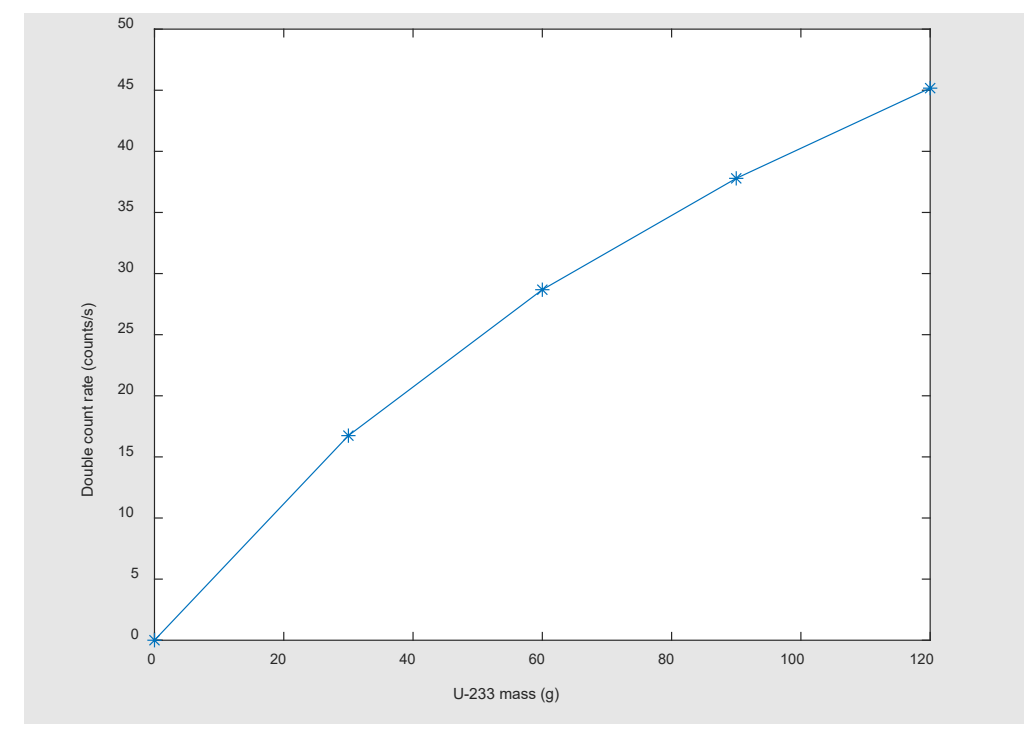

Figure 74. Doubles count rate vs. ${ }^{233} \mathrm{U}$ mass. Error bars are too small to see on the plot.

\subsubsection{MCNP simulations: Passive and Active Assay of Mixed Pu/ ${ }^{232}$ Th Samples Representative of the Identified Thorium Fuel Compositions}

The following MOX fuel compositions were identified in Section 2 as some of the leading options for thorium-fueled reactors (Table 50). In particular, these results correspond to the AHWR used for Stage 3 of India's proposed power program, which represents the multistage fuel cycle in this report. These example fresh fuel compositions rely on plutonium as the fissile start-up material. As discussed in Section 2.3.2.2, the AHWR transition core is the approximate loading in transition from the initial fuel loading to the equilibrium fuel loading. The AHWR has three rings of fuel clusters, which are labeled here as the inner, middle, and outer rings. 
Table 50. Selected Th-Pu fresh fuel compositions.

\begin{tabular}{|c|c|c|c|c|c|c|c|}
\hline Fuel Composition & NatTh & Total Pu & ${ }^{238} \mathrm{Pu}$ & ${ }^{239} \mathrm{Pu}$ & ${ }^{240} \mathrm{Pu}$ & ${ }^{241} \mathrm{Pu}$ & ${ }^{242} \mathrm{Pu}$ \\
\hline Transition Core Inner Ring & $91.0 \%$ & $9 \%$ & $0.095 \%$ & $67.99 \%$ & $24.59 \%$ & $6.005 \%$ & $1.324 \%$ \\
\hline Transition Core Middle Ring & $94.25 \%$ & $5.75 \%$ & $0.095 \%$ & $99 \%$ & $24.59 \%$ & $6.005 \%$ & $1.324 \%$ \\
\hline Transition Core Outer Ring & $97.0 \%$ & $3 \%$ & $0.095 \%$ & $67.99 \%$ & $24.59 \%$ & $6.005 \%$ & $1.324 \%$ \\
\hline Equilibrium Core Inner Ring & $94.0 \%$ & $6 \%$ & $0.095 \%$ & $67.99 \%$ & $24.59 \%$ & $6.005 \%$ & $1.324 \%$ \\
\hline Equilibrium Core Middle Ring & $96.1 \%$ & $3.9 \%$ & $0.095 \%$ & $67.99 \%$ & $24.59 \%$ & $6.005 \%$ & $1.324 \%$ \\
\hline Equilibrium Core Outer Ring & $96.5 \%$ & $2.5 \%$ & $0.095 \%$ & $67.99 \%$ & $24.59 \%$ & $6.005 \%$ & $1.324 \%$ \\
\hline
\end{tabular}

\subsubsection{Passive Assay with ENMC}

This subsection reports on an assessment of the applicability of passive coincidence counting for the fuel compositions listed in Table 50. The ENMC model was used for the simulations. For each fuel composition, $3000 \mathrm{~g}$ of heavy metal are simulated. Both the SFs and the $(a, n)$ contribution due to oxygen were considered. Single and coincidence count rates calculated with MCNP are listed in Table 51, while doubles and triples count rates are shown in Figures 75 and 76 as a function of the equivalent ${ }^{240} \mathrm{Pu}_{\text {eff }}$ mass. As for U-Th fuels, a significant increase in coincidences can be observed as the equivalent ${ }^{240} \mathrm{Pu}_{\text {eff }}$ mass increases. This suggests that passive coincidence techniques are suitable for the assay of Th-Pu fuels, and for the measurement of ${ }^{238} \mathrm{Pu},{ }^{240} \mathrm{Pu}$, and ${ }^{242} \mathrm{Pu}$, due to their high SF probability.

Table 51. Singles, doubles, and triples count rates (ENMC).

\begin{tabular}{|l|c|c|c|}
\multicolumn{1}{|c|}{ Composition } & $\begin{array}{c}\text { Singles (counts/s) } \\
\text { (\% relative error) }\end{array}$ & $\begin{array}{c}\text { Doubles (counts/s) } \\
\text { (\% relative error) }\end{array}$ & $\begin{array}{c}\text { Triples (counts/s) } \\
\text { (\% relative error) }\end{array}$ \\
\hline Transition Core Inner Ring & 64916.14 & 20805.8 & 5581.352 \\
& $(0.22)$ & $(0.79)$ & $(2.18)$ \\
\hline Transition Core Middle Ring & 41016.2 & 12619.5 & 3070 \\
& $(0.22)$ & $(0.79)$ & $(2.20)$ \\
\hline Transition Core Outer Ring & 21199.6 & 6299.73 & 1404 \\
& $(0.22)$ & $(0.79)$ & $(2.20)$ \\
\hline Equilibrium Core Inner Ring & 42833.6 & 13220.5 & 3242 \\
& $(0.22)$ & $(0.79)$ & $(2.28)$ \\
\hline Equilibrium Core Middle Ring & 27646.2 & 8312.6 & 1908 \\
& $(0.22)$ & $(0.79)$ & $(2.20)$ \\
\hline Equilibrium Core Outer Ring & 24774.0 & 7412.5 & 1681 \\
& $(0.22)$ & $(0.79)$ & $(2.20)$ \\
\hline
\end{tabular}




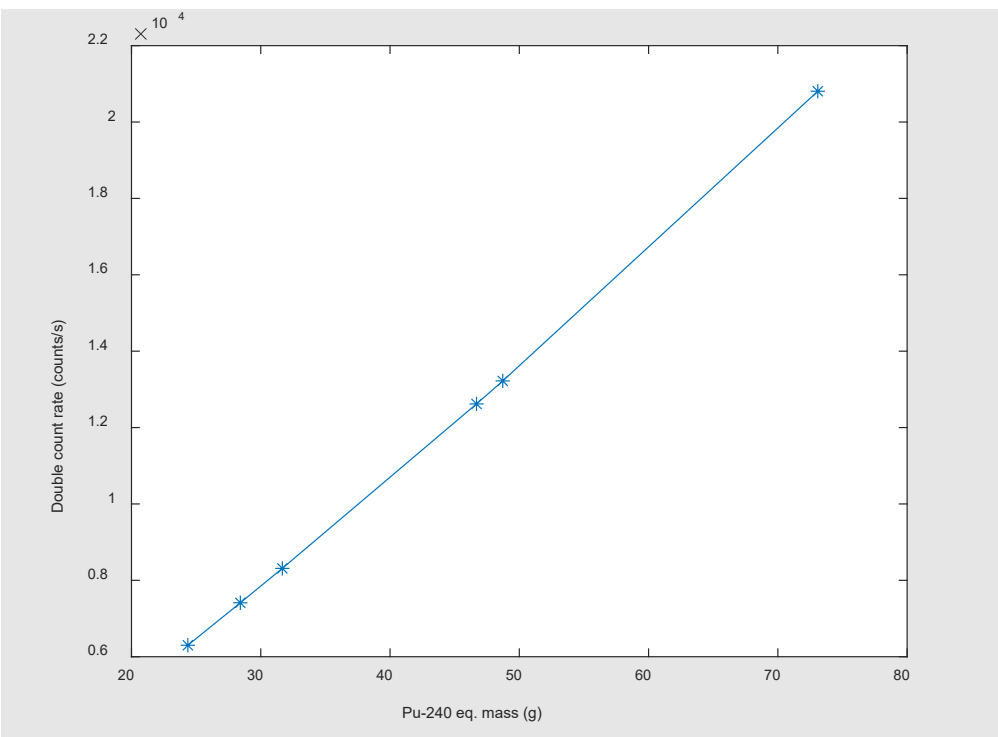

Figure 75. Doubles count rate vs. equivalent ${ }^{240} \mathrm{Pu}_{\text {eff }}$ mass.

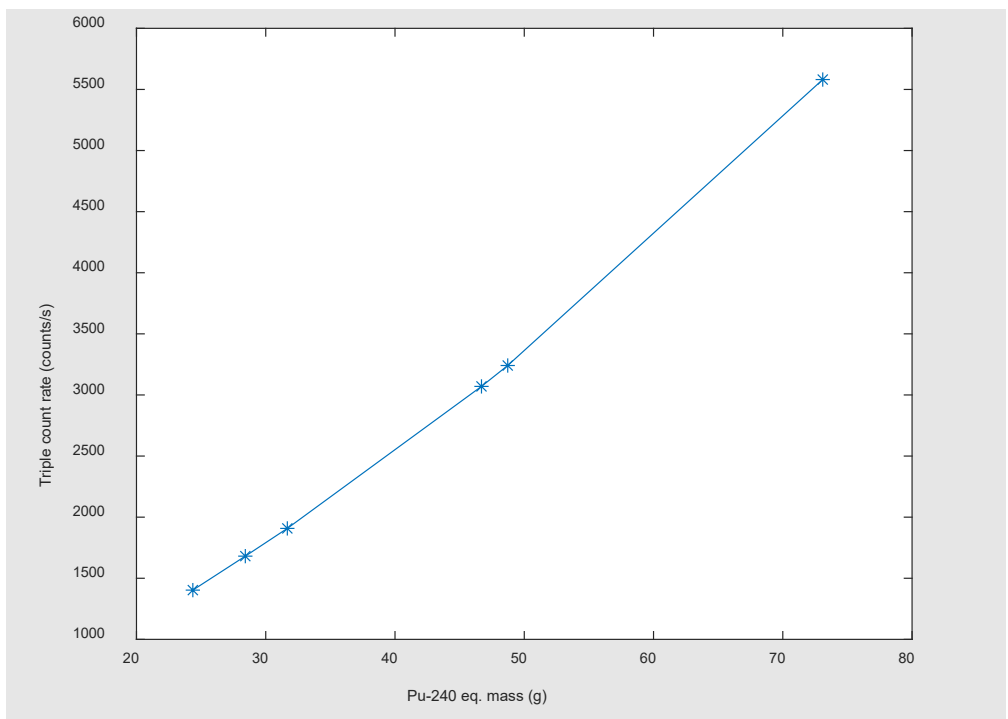

Figure 76. Triples count rate vs. equivalent ${ }^{240} \mathrm{Pu}_{\text {eff }}$ mass.

\subsubsection{Active Assay with AWCC}

This subsection reports on an assessment of active coincidence counting of the six reactor core compositions using the AWCC model. The AmLi Source 1 was used to interrogate the samples. Again, $3000 \mathrm{~g}$ heavy metal (plus oxygen) samples are adopted for each fuel composition. Table 52 lists the singles and coincidence count rates calculated with MCNP, while doubles and triples count rates as a function of the ${ }^{239} \mathrm{Pu}_{\text {eff }}$ mass are shown in Figures 77 and 78 . Additionally, in this case, doubles and triples count rates visibly increase with the content of ${ }^{239} \mathrm{Pu}$, highlighting that active coincidence counting may be a suitable option to assess the fissile content in Th-Pu-based fuels. 
Table 52. Singles, doubles, and triples count rates (ENMC).

\begin{tabular}{|l|c|c|c|}
\multicolumn{1}{|c|}{ Composition } & $\begin{array}{c}\text { Singles (counts/s) } \\
\text { (\% relative error) }\end{array}$ & $\begin{array}{c}\text { Doubles (counts/s) } \\
\text { (\% relative error) }\end{array}$ & $\begin{array}{c}\text { Triples (counts/s) } \\
\text { (\% relative error) }\end{array}$ \\
\hline Transition Core Inner Ring & 2595.6 & 27.9 & 3.19 \\
& $(0.14)$ & $(1.83)$ & $(6.20)$ \\
\hline Transition Core Middle Ring & 2564.7 & 21.4 & 2.82 \\
& $(0.14)$ & $(2.17)$ & $(7.10)$ \\
\hline Transition Core Outer Ring & 2529.7 & 12.6 & 1.31 \\
& $(0.14)$ & $(2.70)$ & $(8.20)$ \\
\hline Equilibrium Core Inner Ring & 2567.3 & 21.8 & 2.74 \\
& $(0.14)$ & $(2.11)$ & $(7.40)$ \\
\hline Equilibrium Core Middle Ring & 2544.1 & 15.6 & 1.57 \\
& $(0.14)$ & $(2.41)$ & $(8.00)$ \\
\hline Equilibrium Core Outer Ring & 2539.0 & 14.2 & 1.46 \\
& $(0.14)$ & $(2.50)$ & $(7.40)$ \\
\hline
\end{tabular}

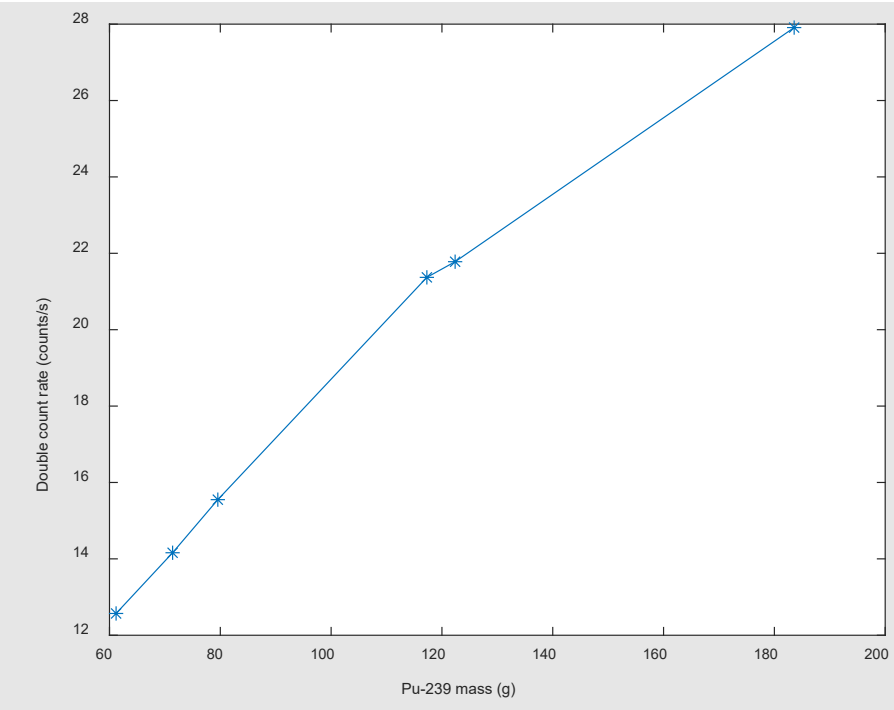

Figure 77. Doubles count rate vs. ${ }^{239}$ Pu mass.

\subsection{Key Observations and Findings}

\subsubsection{Conclusions from Neutron Measurement Simulations and Experiments}

Several representative active and passive coincidence counting techniques currently available for assay of U-Pu nuclear fuels were investigated to assess their applicability to fuels found within the thorium fuel cycles. After benchmarking the AWCC and the ENMC MCNP models using plutonium oxide, $\mathrm{AmLi}$, and ${ }^{252} \mathrm{Cf}$ sources, the assays of several thorium samples were simulated. The following conclusions can be drawn:

- Passive neutron coincidence counting is not suitable for the assay of ${ }^{232} \mathrm{Th}$, whether isolated or in a mixture, due to the low SF probability of this nuclide. 


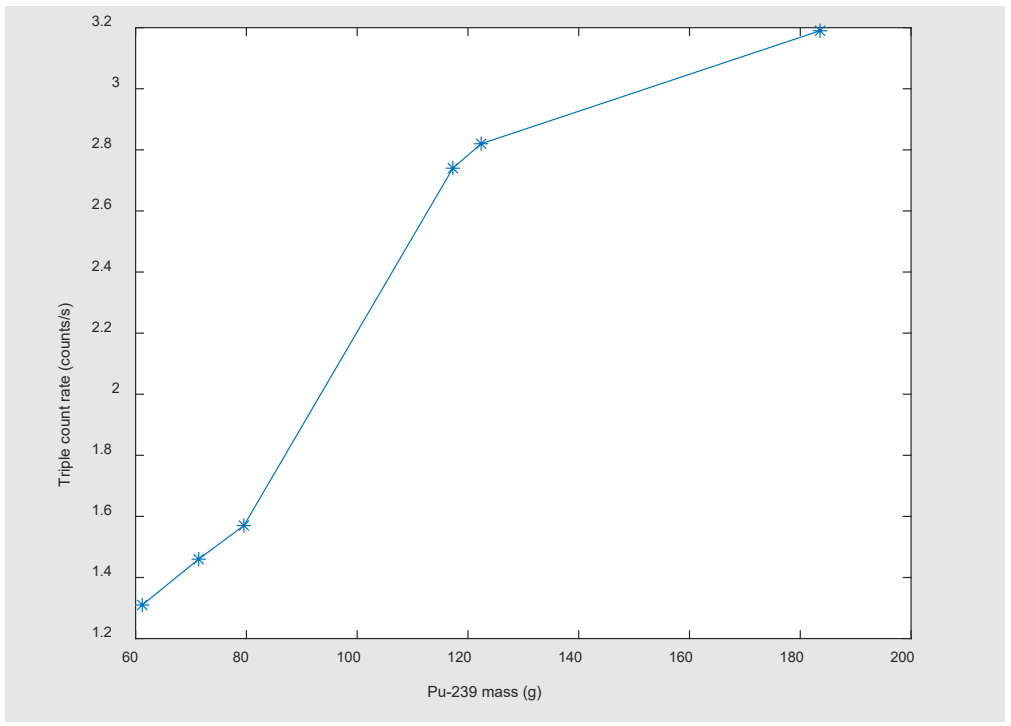

Figure 78. Triples count rate vs. ${ }^{239} \mathrm{Pu}$ mass.

- Active neutron coincidence counting is not a practical option for the assay of ${ }^{232} \mathrm{Th}$, whether isolated or in a mixture, or whether using AmLi or ${ }^{252} \mathrm{Cf}$ interrogation sources. No significant differences were observed in singles and coincidence count rates between measurements carried out with or without (at background) thorium samples. This suggests that virtually all the neutrons are emitted by the interrogation sources instead of thorium.

- Active neutron coincidence counting with the AWCC is suitable to measure the ${ }^{233} \mathrm{U}$ content in thorium-based fuels.

- Both active and passive neutron coincidence counting can be applied to the assay of Th-Pu fuels. Active techniques are suitable for the assay of the fissile isotopes of plutonium, while passive techniques can be applied to the assay of ${ }^{238} \mathrm{Pu}$, ${ }^{240} \mathrm{Pu}$, and ${ }^{242} \mathrm{Pu}$, due to the high SF probability of these nuclides.

\subsubsection{Feasibility of Neutron NDA Techniques for ${ }^{233} \mathrm{U}$ - and Thorium-bearing Material}

The discussion in previous sections has attempted to identify and, to a limited extent validate, potential modes of NDA for items containing ${ }^{232} \mathrm{Th}$ and/or ${ }^{233} \mathrm{U}$ in various chemical and physical forms. Evaluations were based primarily on assessing fundamental physical and chemical properties of both isotopes and their various chemical forms, and on drawing analogies with the body of experience with established NDA methods for the assay of uranium and plutonium in conventional U/Pu fuel cycles.

Table 53 provides an overview of these findings, in addition to extrapolating findings for nuclear material that was not simulated nor measured, in terms of the anticipated feasibility of performing safeguards measurements, where the measurement objective is to verify the consistency of neutron signatures with declarations, including nuclear material composition and mass, in reasonable time frames (i.e., several minutes to approximately an hour). Overall, it appears that the quantitative assay of ${ }^{232}$ Th relying on neutron signatures is difficult, and, in many instances, seems impractical with any established methods. Quantitative assay of ${ }^{233} \mathrm{U}$ appears, in principle, to be possible with a variety of instruments already adopted for the NDA of uranium- and plutonium-bearing items in established 
safeguards procedures. Moreover, the appropriate combination and application of certain NDA methods may even yield the capability to discriminate between ${ }^{233} \mathrm{U}$ and ${ }^{235} \mathrm{U}$ from neutron signatures alone.

We recommend that future work include verifying conclusions shown in Table 53 by means of dedicated experiments or high-fidelity simulations as an initial phase of any R\&D effort focused on safeguards technology development for thorium-based nuclear fuel cycles.

Table 53. Summary of the evaluation of the feasibility of neutron NDA techniques.

\begin{tabular}{|c|c|c|c|c|c|}
\hline $\begin{array}{l}\text { Assay } \\
\text { target }\end{array}$ & Material type & $\begin{array}{c}\text { Passive } \\
\text { assay }\end{array}$ & Active assay & Self-interrogation & Note \\
\hline \multirow[t]{7}{*}{${ }^{232} \mathrm{Th}$} & ${ }^{232}$ Th metal & $\begin{array}{c}\text { Not } \\
\text { feasible }\end{array}$ & $\begin{array}{c}\text { Difficult, fast neutrons } \\
\text { only }(>1.5 \mathrm{MeV})\end{array}$ & Not feasible & \\
\hline & $\mathrm{ThO}_{2}$ & $\begin{array}{c}\text { Not } \\
\text { feasible }\end{array}$ & $\begin{array}{l}\text { Difficult, fast neutrons } \\
\text { only }(>1.5 \mathrm{MeV})\end{array}$ & Not feasible & \\
\hline & $\left({ }^{\text {nat }} \mathrm{U}, \mathrm{Th}\right) \mathrm{O}_{2}{ }^{*}$ & $\begin{array}{c}\text { Not } \\
\text { feasible }\end{array}$ & Not feasible & Not feasible & $\begin{array}{c}\text { Th signature likely } \\
\text { masked by natU signature }\end{array}$ \\
\hline & $\left({ }^{233} \mathrm{U}, \mathrm{Th}\right) \mathrm{O}_{2}$ & $\begin{array}{c}\text { Not } \\
\text { feasible }\end{array}$ & Not feasible & Not feasible & $\begin{array}{l}\text { Th signature likely } \\
\text { masked by }{ }^{233} \mathrm{U} \\
\text { signature }\end{array}$ \\
\hline & $\begin{array}{c}\text { (enriched U, } \\
\text { Th) } \mathrm{O}_{2}{ }^{*}\end{array}$ & $\begin{array}{c}\text { Not } \\
\text { feasible }\end{array}$ & Not feasible & Not feasible & $\begin{array}{c}\text { Th signature likely } \\
\text { masked by U signature }\end{array}$ \\
\hline & ThC $^{*}$ & $\begin{array}{c}\text { Not } \\
\text { feasible }\end{array}$ & $\begin{array}{l}\text { Difficult, fast neutrons } \\
\text { only }(>1.5 \mathrm{MeV})\end{array}$ & Not feasible & \\
\hline & $\begin{array}{l}\text { Th(FLiBe) } \\
\text { salt }^{*}\end{array}$ & $\begin{array}{c}\text { Not } \\
\text { feasible }\end{array}$ & $\begin{array}{l}\text { Difficult, fast neutrons } \\
\text { only }(>1.5 \mathrm{MeV})\end{array}$ & Not feasible & \\
\hline \multirow[t]{3}{*}{${ }^{233} \mathrm{U}$} & ${ }^{233} \mathrm{U}$ metal & $\begin{array}{c}\text { Not } \\
\text { feasible }\end{array}$ & $\begin{array}{l}\text { Feasible, similar to } \\
{ }^{235} \mathrm{U}\end{array}$ & Not feasible & \\
\hline & ${ }^{233} \mathrm{UO}_{2}$ & $\begin{array}{c}\text { Not } \\
\text { feasible }\end{array}$ & $\begin{array}{l}\text { Feasible, similar to } \\
{ }_{235} \mathrm{U}\end{array}$ & $\begin{array}{c}\text { Difficult, potentially } \\
\text { feasible }\end{array}$ & \\
\hline & ${ }^{233} \mathrm{UF}_{4}$ & $\begin{array}{c}\text { Not } \\
\text { feasible }\end{array}$ & $\begin{array}{l}\text { Feasible, similar to } \\
{ }^{235} \mathrm{U}\end{array}$ & Likely feasible & \\
\hline
\end{tabular}

*Material type not included in the simulations or measurements.

\subsection{Gamma Signatures}

Regardless of the decay mode of an unstable atomic nucleus, in nearly all cases, the emission of an aor $\beta$-particle is accompanied by emission of one or more gamma rays. That is due to the nature of the process, wherein the daughter nucleus is most often formed in an excited state and, to reach its ground state, one or more gamma rays must be emitted in short succession, carrying away the excess energy. Moreover, as the discrete nature of energy levels of these excited states and the branching ratios of possible transitions are ruled by the internal, isotope-specific structure of the nucleus, the resulting energy spectrum of emitted gamma rays gives rise to a fingerprint that is unique to each radioactive isotope. 
This illustrates how detecting gamma radiation from a radioactive source can be used to determine its isotopic composition and thus complement information from neutron signatures related to the item's properties, such as mass. However, not all isotopes produce easily measurable gamma rays, and in some instances, only gamma spectra from decay of secondary or tertiary products can provide evidence of a certain isotope's presence within the assayed item. Moreover, additional engineering requirements, such as those tied to safe handling of a particular item (e.g., shielding), may hamper measurement of gamma rays of certain energy or with sufficient resolution. Unfortunately, this is a common scenario regarding items and materials relevant in thorium-based nuclear fuel cycles.

The following discussion is not an exhaustive review of potential pitfalls of gamma spectroscopic measurements involving ${ }^{232} \mathrm{Th}$ and ${ }^{233} \mathrm{U}$, but rather a general outline of the most common and prevalent issues that may at times make gamma-based NDA of thorium fuel cycle-related items an extraordinary challenge.

\subsubsection{Production and Decay of ${ }^{233} U$ and ${ }^{232} \mathrm{U}$}

${ }^{233} \mathrm{U}$ is produced when ${ }^{232} \mathrm{Th}$ is subjected to neutron irradiation, such as within a thorium-based nuclear reactor (Figure 79). Albeit through a different chain of nuclear reactions, ${ }^{232} \mathrm{U}$ is created concurrently. As the fuel burnup increases, the concentration of ${ }^{232} \mathrm{U}$ increases faster than the ${ }^{233} \mathrm{U}$ concentration, even though on an absolute scale it remains typically three to five orders of magnitude lower.

In thorium-based nuclear fuel cycles, the production and subsequent extraction of ${ }^{233} \mathrm{U}$ is, at least in some stages, one of the primary objectives. However, the mode of extraction determines the properties of the fissile uranium (e.g., material form, isotopic vector) from the perspectives of nuclear safeguards.

For example, in MSRs with continuous recycling and reprocessing of fuel, protactinium is chemically separated from the molten salt, which beta-decays into ${ }^{233} \mathrm{U}$ with only minor contamination by ${ }^{232} \mathrm{U}$. Should uranium be reprocessed from spent thorium fuel after discharge from the reactor, the concentration of ${ }^{232} \mathrm{U}$ contaminant may be at sufficient levels to cause safety-related challenges that require heavy shielding and ultimately prevent deployment of many traditional gamma-based NDA instruments and methods. Following discharge and chemical separation, essentially only ${ }^{233} \mathrm{U}$ $\left(\mathrm{T}_{1 / 2}=1.58 \times 10^{5} \mathrm{y}\right)$ and ${ }^{232} \mathrm{U}\left(\mathrm{T}_{1 / 2}=68.9 \mathrm{y}\right)$ isotopes are left. Due to its short half-life, the progeny of ${ }^{232} \mathrm{U}$ build up quickly, not only dominating the gamma ray signature of materials bearing such reprocessed uranium but also yielding an ionizing radiation dose that may cause substantial risks to the environment and human operators (Figure 80). 


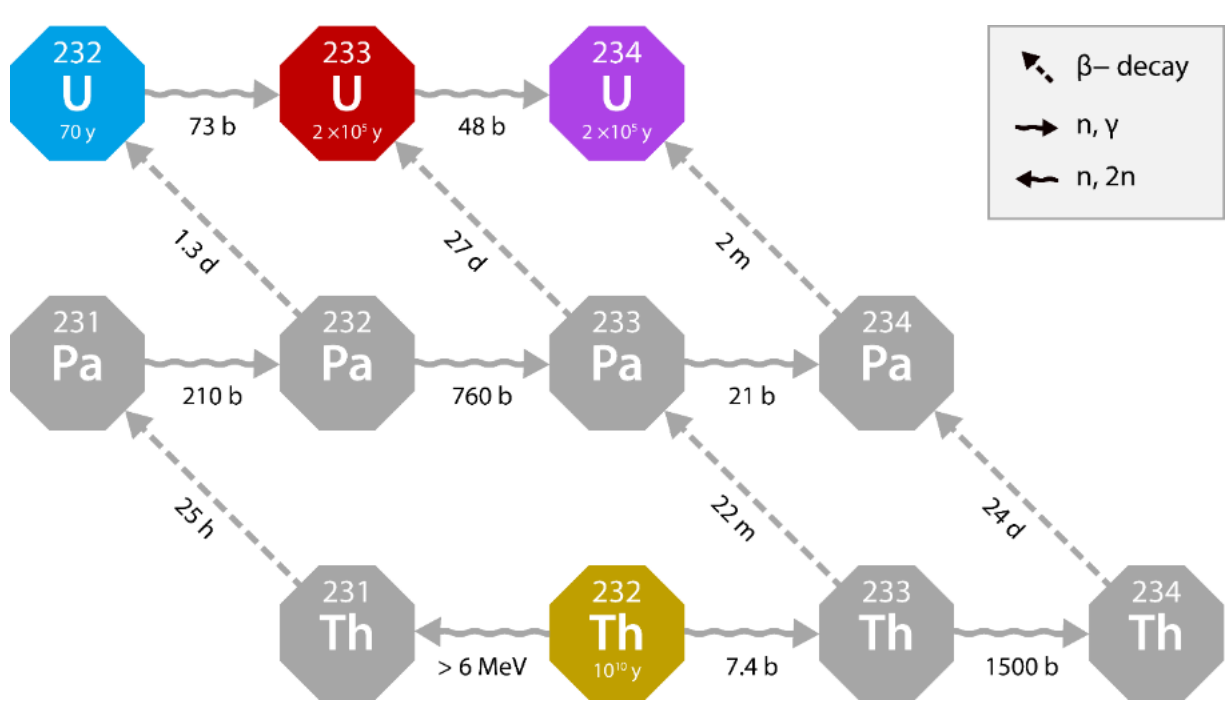

Figure 79. Production of ${ }^{233} \mathrm{U}$ and ${ }^{232} \mathrm{U}$ in a thorium fuel-based nuclear reactor.

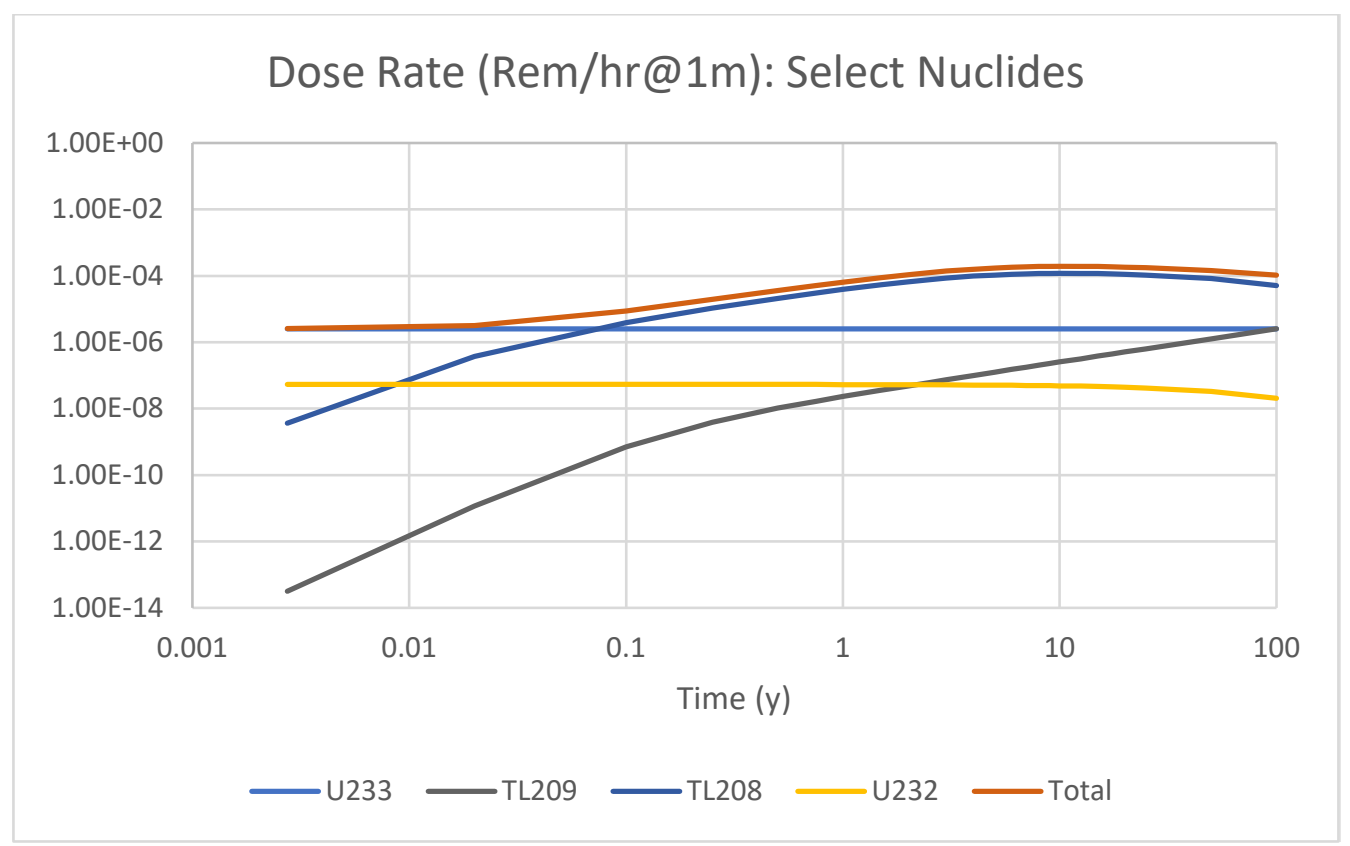

Figure 80. Dose rate from a 1 gram ${ }^{233} \mathrm{U}$ item with $10 \mathrm{ppm}{ }^{232} \mathrm{U}$, ignoring self-attenuation. Despite the low mass and relative isotopic purity, this item reaches $0.1 \mathrm{mrem} / \mathrm{hr}$ at $1 \mathrm{~m}$ (or equivalently $1 \mathrm{rem} / \mathrm{hr}$ at $1 \mathrm{~cm}$ ) after $\sim 10$ years of aging.

This dose is mostly due to the ingrowth of ${ }^{208} \mathrm{Tl}$ in the ${ }^{232} \mathrm{U}$ decay chain, as shown in Figure 81 . Moreover, because a large fraction of this dose comes from the $2614 \mathrm{keV}$ gamma ray, the materials with substantial ${ }^{232} \mathrm{U}$ contamination require large amounts of shielding to significantly reduce the dose rates. It must be noted that $\sim 11 \mathrm{~mm}$ of lead shielding is required to reduce such a dose by a factor of two. 


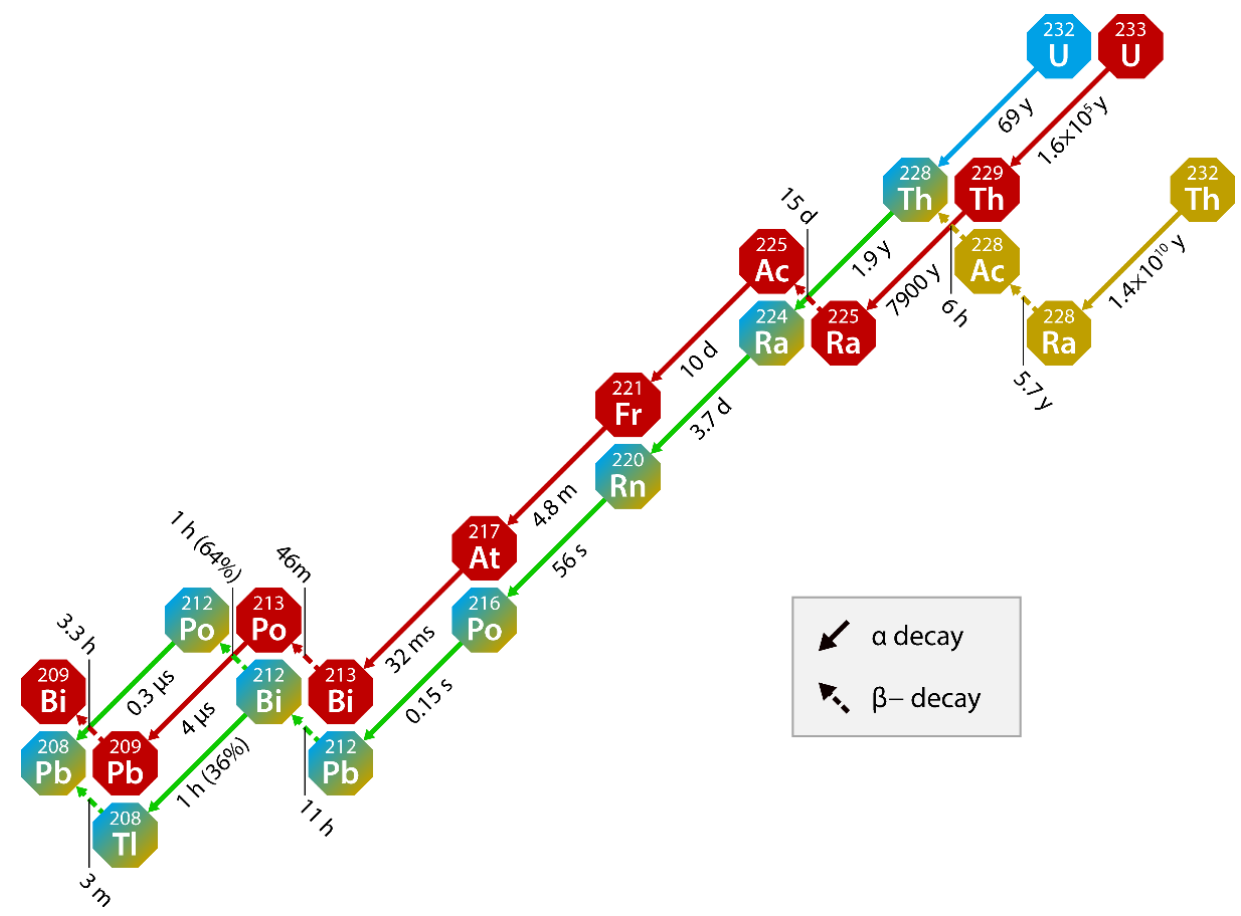

Figure 81. Decay chains of ${ }^{233} U,{ }^{232} T h$, and ${ }^{232} U$. See also Ref. [72].

\subsubsection{Dose Rates and Shielding Considerations Related to ${ }^{232} \mathrm{U}$ Contamination}

As an explicit example including the effects of self-attenuation, a $1 \mathrm{~kg}$ sphere of ${ }^{233} \mathrm{U}$ with assumed density of $15 \mathrm{~g} \cdot \mathrm{cm}^{-3}$ would have a radius of $2.52 \mathrm{~cm}$. Further assuming $10 \mathrm{ppm}$ of ${ }^{232} \mathrm{U}$ contamination and a period of 5 years of aging since reprocessing, this item produces a dose rate of $52.6 \mathrm{mrem} / \mathrm{hr}$ at $1 \mathrm{~m}$ (measured from the center of the item). To reduce the dose rate to $10 \mathrm{mrem} / \mathrm{hr}$ at $1 \mathrm{~m}$ would require $3.5 \mathrm{~cm}$ of lead shielding, with a minimum mass of $9 \mathrm{~kg}$ of lead depending on the shielding geometry.

However, in many instances, $10 \mathrm{ppm}{ }^{232} \mathrm{U}$ concentrations can be considered on the lower end of a potential concentration range. For example, many of the ${ }^{233} \mathrm{U}$ items housed at ORNL have ${ }^{232} \mathrm{U}$ concentrations well over $100 \mathrm{ppm}$. Repeating the calculation for a geometrically similar sphere, but with an increase in the contamination level from 10 to $150 \mathrm{ppm}$ of ${ }^{232} \mathrm{U}$, results in an unshielded dose rate of $787 \mathrm{mrem} / \mathrm{hr}$ at $1 \mathrm{~m}$. To reduce the dose rate to $10 \mathrm{mrem} / \mathrm{hr}$ at $1 \mathrm{~m}$ would require $9.95 \mathrm{~cm}$ of lead shielding, for a total minimum mass of $87.4 \mathrm{~kg}$ of lead.

As expected, the dose rates from such materials present a significant safety and subsequent handling challenge. As demonstrated in Table 54, workers could easily reach their yearly DOE occupational dose limit of 5 rem within a few hours of working with these materials. Consequently, access to such materials and conduct of operations typical for many safeguards inspections across the U/Pu fuel cycle is likely to be impacted by either the presence of heavy shielding or requirements to perform the assay of relevant items remotely, each of these aspects posing its own unique challenges. 
Table 54. Unshielded working hours required to accumulate a $5 \mathrm{rem}$ dose (5 $\mathrm{kg}$ sphere of metal at $50 \mathrm{~cm}$ ) one year after separation [73].

\begin{tabular}{|c|c|c|}
\hline Metal & Dose Rate (rem/hr) & Hours \\
\hline Weapon-grade plutonium & 0.0013 & 3800 \\
\hline Reactor-grade plutonium & 0.0082 & 610 \\
\hline${ }^{233} \mathrm{U}$ containing $1 \mathrm{ppm}{ }^{232} \mathrm{U}$ & 0.013 & 380 \\
\hline${ }^{233} \mathrm{U}$ containing $5 \mathrm{ppm}{ }^{232} \mathrm{U}$ & 0.059 & 80 \\
\hline${ }^{233} \mathrm{U}$ containing $100 \mathrm{ppm}{ }^{232} \mathrm{U}$ & 1.27 & 4 \\
\hline${ }^{233} \mathrm{U}$ containing $1 \%{ }^{232} \mathrm{U}$ & 127 & 0.04 \\
\hline
\end{tabular}

\subsubsection{Gamma Ray Signatures}

While the mechanical aspects of the ${ }^{233} \mathrm{U}$-bearing item container, as well as its physical and isotopic parameters, are important to determining how safeguards inspection may proceed, the prospect of successful NDA is still derived from the isotope's most fundamental nuclear properties, namely, its isotope-specific gamma spectra. Despite ${ }^{232} \mathrm{U}$ being a rather small impurity by weight, gamma rays from its progeny dominate the gamma ray spectra for all but freshly processed ${ }^{233} \mathrm{U}$ items. Once the ${ }^{232} \mathrm{U}$ progeny grows in, the gamma ray signature is similar to the signature from ${ }^{232} \mathrm{Th}$ decay chain. The main difference is the absence of ${ }^{228} \mathrm{Ac}$ in the ${ }^{232} \mathrm{U}$-decay chain, which contributes several significant gamma rays, particularly those at $338 \mathrm{keV}, 911 \mathrm{keV}$, and $969 \mathrm{keV}$, as shown in Figure 82, and typically allows for reliable discrimination of ${ }^{232} \mathrm{U}$ from ${ }^{232} \mathrm{Th}$.

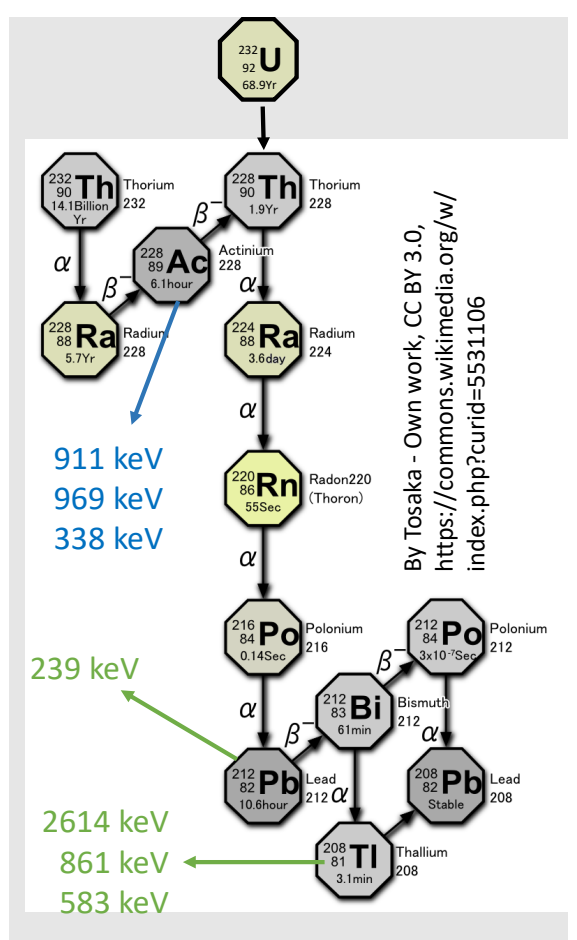

Figure 82. Annotated decay chains of ${ }^{232} \mathrm{Th}$ and ${ }^{232} \mathrm{U}$. Image modified from Ref. [74]. 
Using the $911 / 969 \mathrm{keV}$ complex to distinguish between ${ }^{232} \mathrm{U}$-containing items and ${ }^{232} \mathrm{Th}$ is straightforward, even for low-resolution detectors, as demonstrated in Figure 83. While this distinction is relatively simple for human spectroscopists, automated radioisotope identifiers (RIIDs), such as FLIR's identiFINDER, deliver significantly less reliable performance, often simply yielding "232 $\mathrm{U} /{ }^{232} \mathrm{Th}$ " as a result when used in the field [75]. This is due to the nature of the RIID libraries, so they would have to be updated to handle this nuance.

When measuring small or trace quantities of ${ }^{233} \mathrm{U}$ in the presence of significant natural background radiation, looking purely at the $911 \mathrm{keV} / 969 \mathrm{keV}$ complex may be impractical due to the low signal-tobackground ratio. However, in such cases, the $2614 \mathrm{keV}$ gamma ray count rate will still be significantly elevated above background, as in Figure 84, particularly for aged materials, providing at least an attribute compatible with certain scenarios warranting further investigation, such as in the case of Complementary Access (CA).

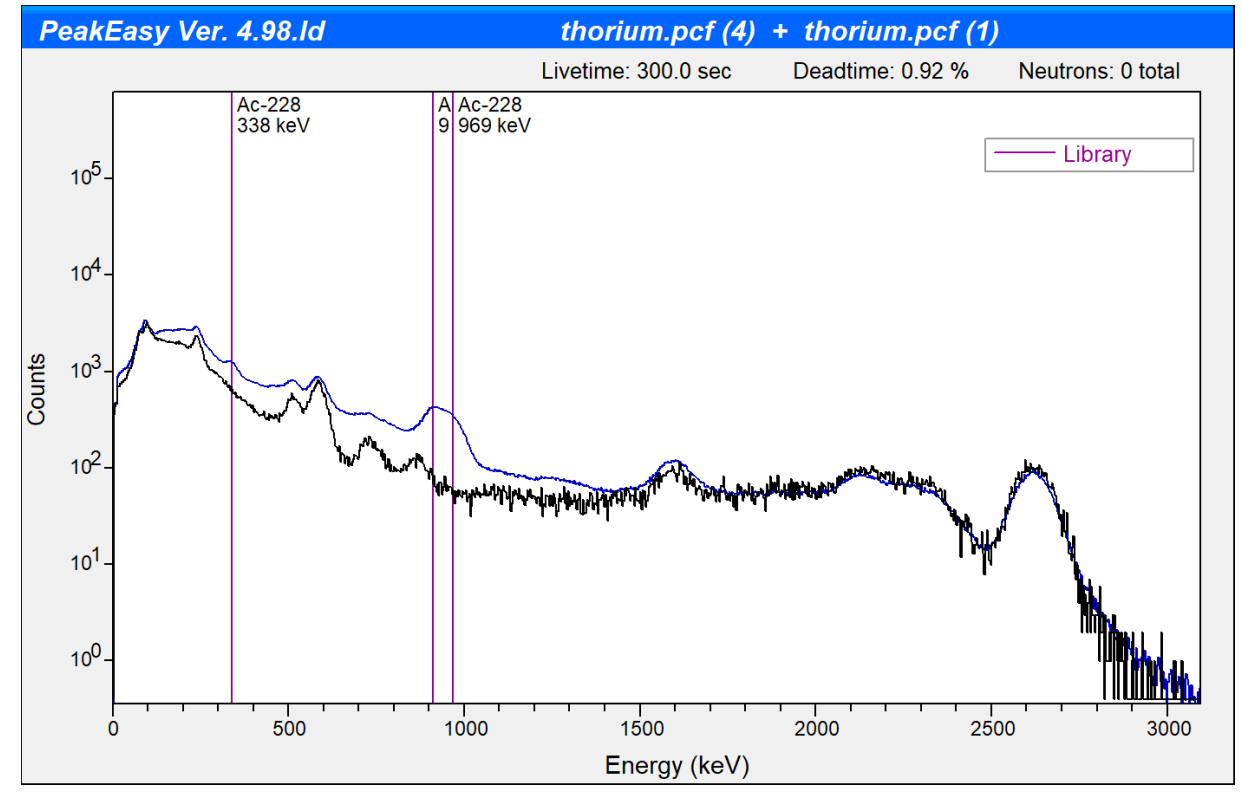

Figure 83. Comparisons of a $1 \mathrm{~kg} \mathrm{ThO_{2 }}$ spectrum (blue) with a $10 \mathrm{~g}^{233} \mathrm{U}$ metal with $10 \mathrm{ppm}{ }^{232} \mathrm{U}$ aged for 6 months spectrum (black), normalized by the $\mathbf{2 6 1 4}$ keV peak area. Spectra were simulated in GADRAS 18.8.1 as if measured with an identiFINDER-NG. The ${ }^{228} \mathrm{Ac}$ lines are strongly visible in the thorium spectrum, making it possible to quickly distinguish between a ${ }^{233} \mathrm{U}$-bearing item and a thorium-bearing item.

However, distinguishing between thorium-bearing and ${ }^{232} \mathrm{U}$-bearing items is not the only NDA application of interest. Another likely application is determining/verifying ${ }^{232} \mathrm{U}$ concentrations in ${ }^{233} \mathrm{U}$, which may enable the IAEA to verify the reactor history of these materials. 


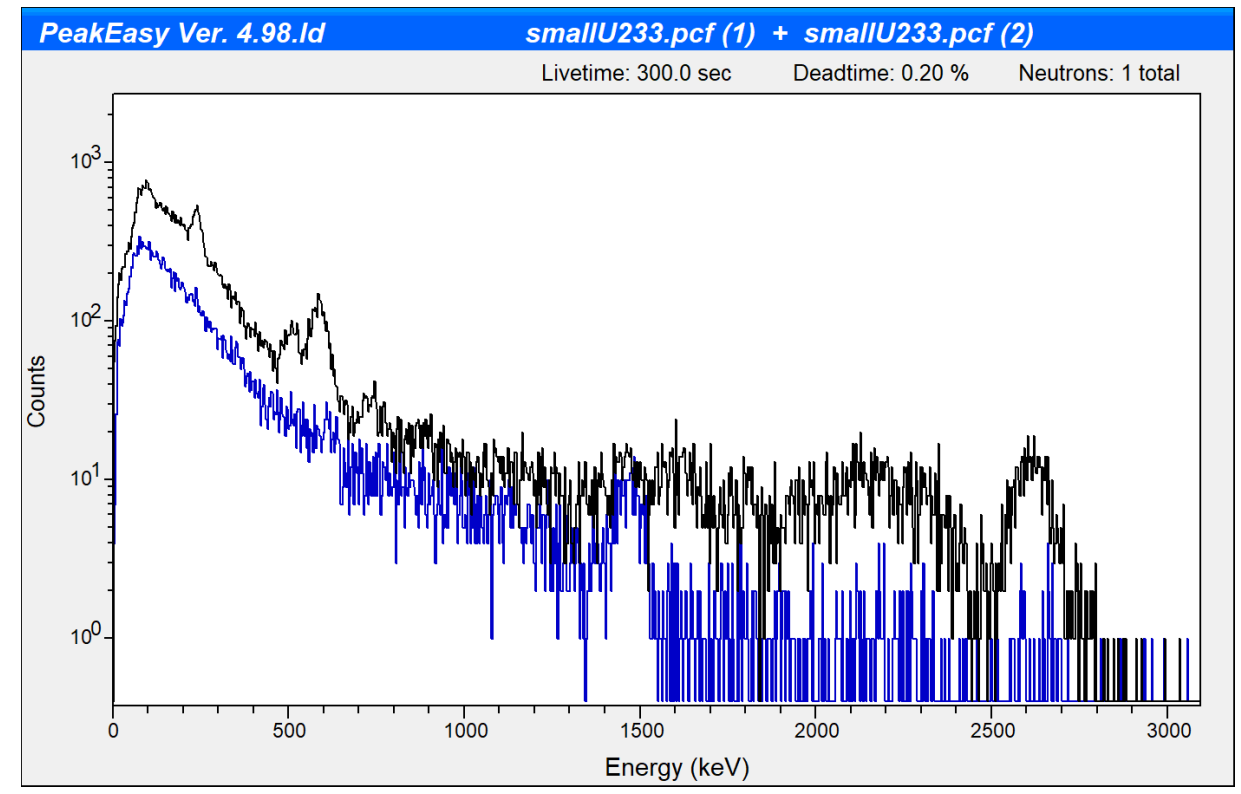

Figure 84. Simulated spectra of a $1 \mathrm{~g}^{233} \mathrm{U}$ source ( $10 \mathrm{ppm}{ }^{233} \mathrm{U}, 6$ months old) (black), measured with an identiFINDER-NG at $100 \mathrm{~cm}$ and natural background (blue). Even for this small amount of relatively pure material, the $2614 \mathrm{keV}$ count rate is significantly increased over background.

Time-separated measurements could be made to determine ${ }^{232} \mathrm{U}$ content. Alternatively, accurate determination of ${ }^{232} \mathrm{U}$ concentration with gamma-based NDA would generally require the following steps:

1. Identify and characterize peaks for all isotopes of interest. This includes not only the direct ${ }^{233} \mathrm{U}$ and ${ }^{232} \mathrm{U}$ gamma rays but also their progeny, which dominate the gamma ray spectrum. In addition, include ${ }^{232} \mathrm{Th}$ and ${ }^{228} \mathrm{Ac}$ to avoid biases from terrestrial background and possible thorium (as part of naturally occurring radioactive material) in the item.

2. Determine the relative efficiency curve for the gamma ray energy range of interest.

3. Determine the efficiency-corrected isotopic ratios for all nuclides of interest.

4. Determine the mass fractions for nuclear material types and compositions of interest.

5. Determine the age of the material (generally from the ${ }^{232} \mathrm{U}$ progeny).

Because only high-resolution gamma ray measurements can be used for this task, the choice of instruments for field assessment of ${ }^{232} \mathrm{U}$ concentrations is restricted to detectors of sufficient resolution, likely disqualifying many widely used handheld units such as the identiFINDER HM-5. Furthermore, we are not aware of any COTS analysis software for this application, although modification of currently used analytical packages such as LANL's FRAM code [76] to include this capability should be straightforward and could expand the applicability of existing analysis packages to thorium fuel cycle safeguards.

In general, the challenges associated with gamma-based identification or characterization of ${ }^{233} \mathrm{U}$ bearing material are significant. Perhaps the most consequential challenge is the expectation that any 
bulk ${ }^{233} \mathrm{U}$ material would be shielded due to the gamma radiation dose rates from the ${ }^{232} \mathrm{U}$ progeny, making the measurement of many of the required gamma rays simply infeasible. Figures 85 and 86 compare simulated gamma spectra of a $1 \mathrm{~kg}$ sphere of ${ }^{233} \mathrm{U}\left(10 \mathrm{ppm}^{232} \mathrm{U}, 1\right.$ year old $)$ with and without (only) $1 \mathrm{~cm}$ of lead shielding.

In the spectra shown in Figures 85 and 86, the signal from the ${ }^{233} \mathrm{U}$ itself is rather difficult to detect. In the shielded measurement, only the weak line from ${ }^{209} \mathrm{Tl}$ at $1567 \mathrm{keV}$ is visible, and accurately fitting this peak would require overall very high statistics (i.e., significantly longer than the target measurement times described above). Figure 87 shows a $24 \mathrm{hr}$ simulation of this same item, which indicates that a $24 \mathrm{hr}$ measurement time would likely be required.

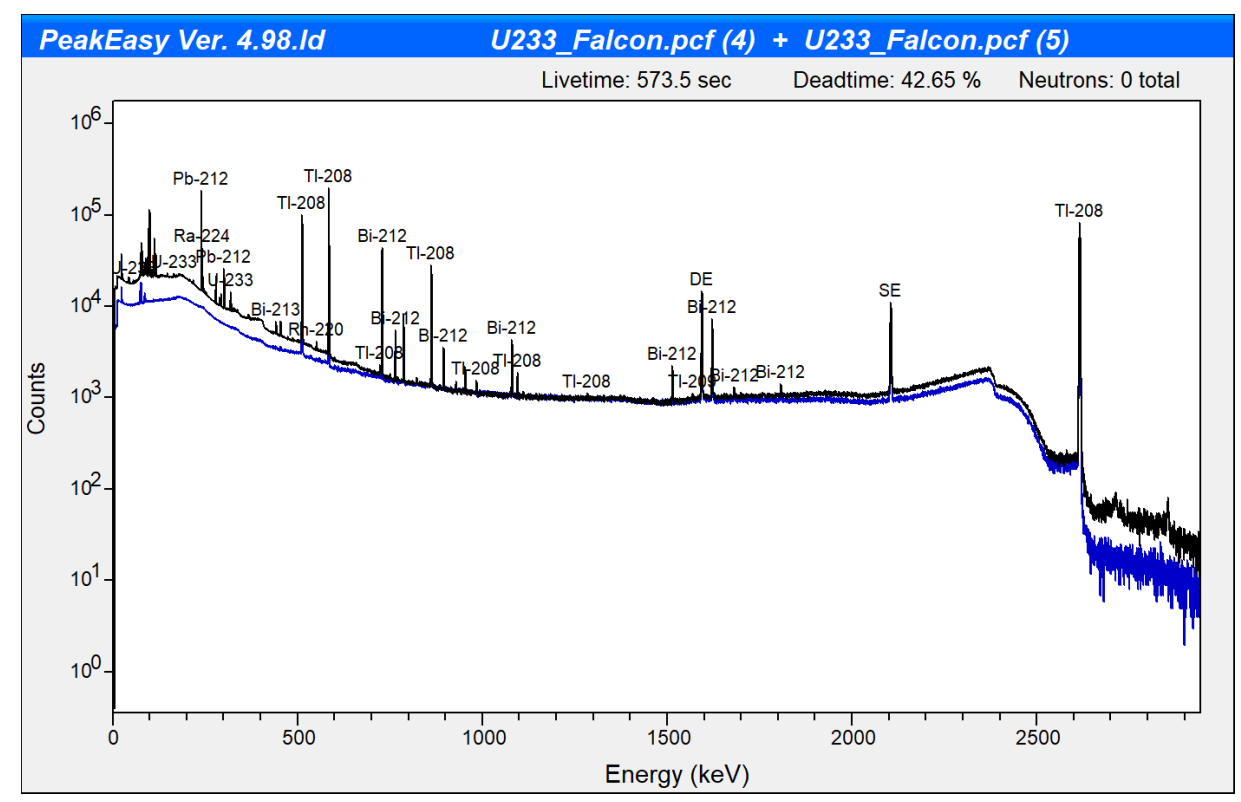

Figure 85. Simulated high-resolution spectra of a $1 \mathrm{~kg}{ }^{233} \mathrm{U}$ item with $10 \mathrm{ppm}{ }^{232} \mathrm{U}$ contamination (aged for 1 year) with $1 \mathrm{~cm}$ of lead shielding (blue) and without the shielding (black). Simulated for a Canberra Falcon 5000 detector $150 \mathrm{~cm}$ from the source.

Because the ${ }^{209} \mathrm{Tl}$ grows in slowly (Figure 88), this peak is more useful with aged material than with more freshly separated material (<10 years). This presents a challenge when measuring ${ }^{233} \mathrm{U}$ items in the first decade, as the activity of ${ }^{208} \mathrm{Tl}$ rapidly increases and obscures all the ${ }^{233} \mathrm{U}$ signatures in shielded items for years. 


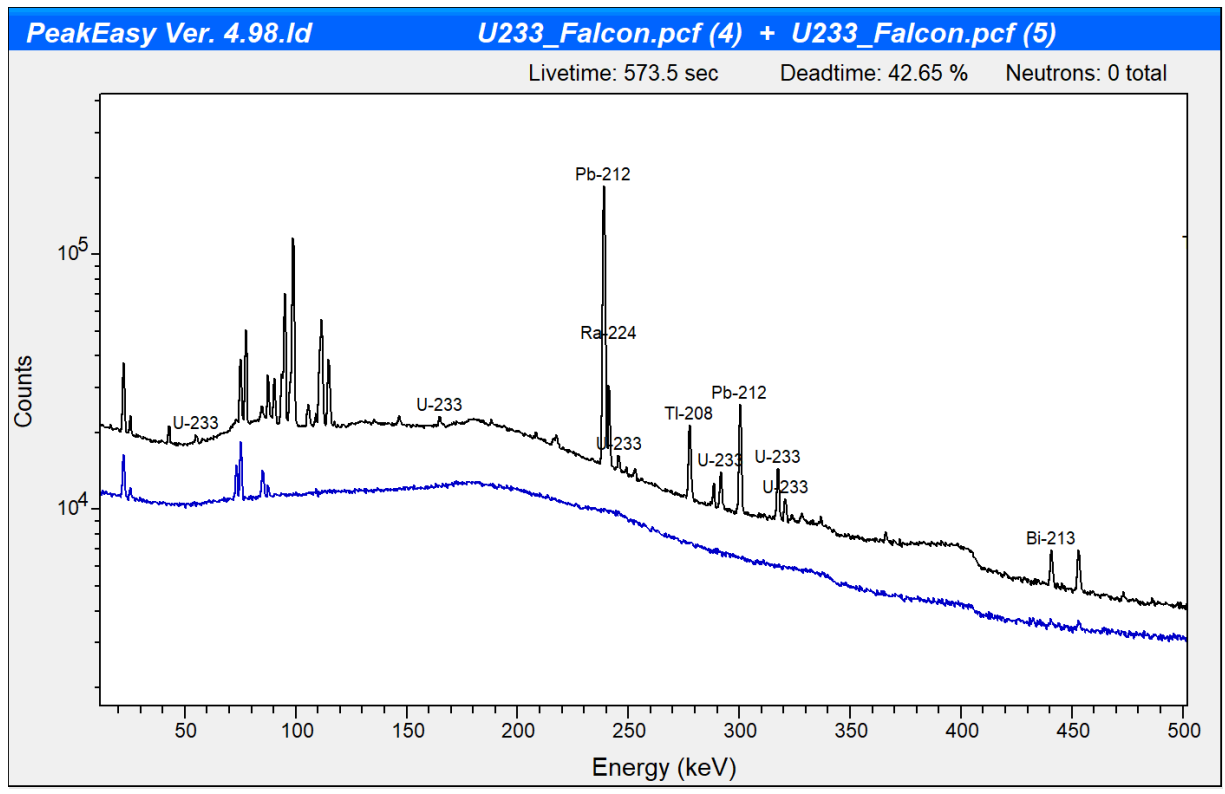

Figure 86. Simulated high-resolution spectra of a $1 \mathrm{~kg}{ }^{233} \mathrm{U}$ item (10 ppm ${ }^{233} \mathrm{U}, 1$ year old) with $1 \mathrm{~cm}$ of lead shielding (blue) and without the shielding (black). Simulated for a Canberra Falcon 5000 detector $150 \mathrm{~cm}$ from the source. Zoomed in for the $0-500 \mathrm{keV}$ region. Note that the ${ }^{233} \mathrm{U}$ item peaks are dominated by the ${ }^{232} \mathrm{U}$ progeny, and in the shielded measurement the peaks from ${ }^{233} \mathrm{U}$ itself are not visible at all.

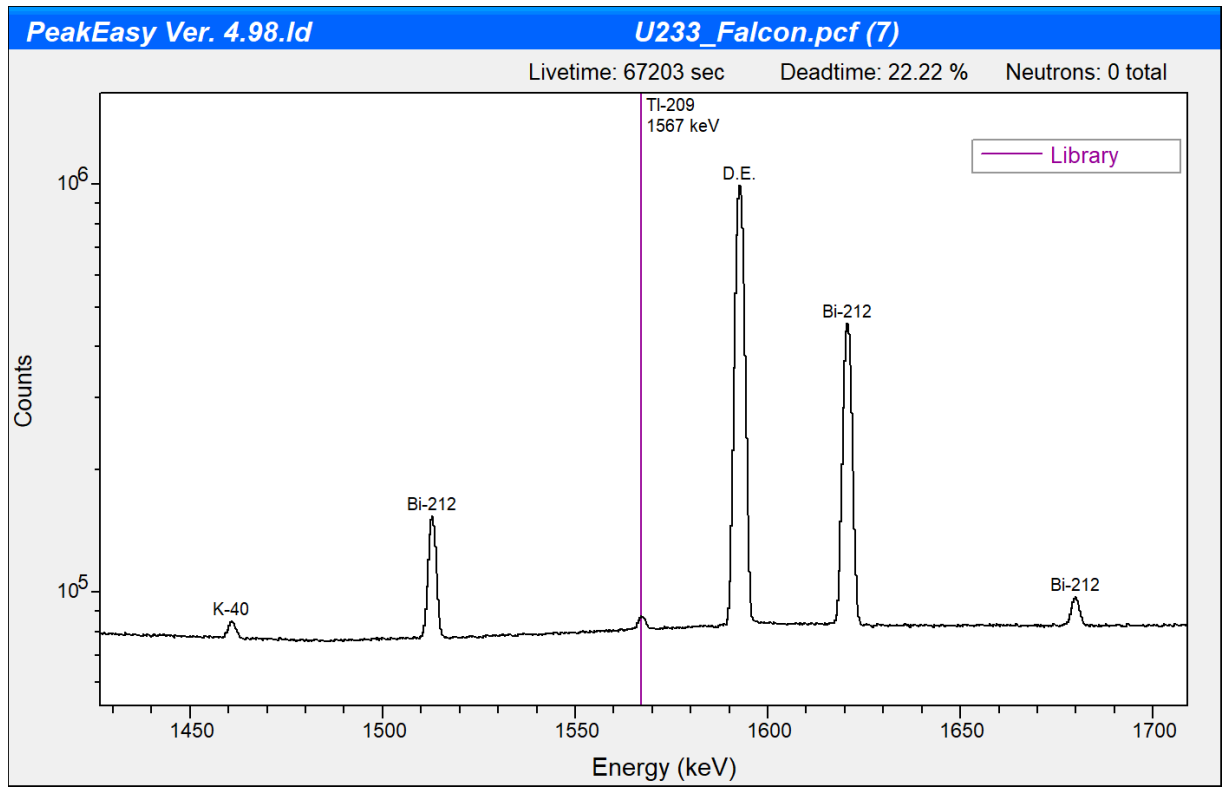

Figure 87. Simulated high-resolution spectra of a $1 \mathrm{~kg}{ }^{233} \mathrm{U}$ item ( $10 \mathrm{ppm}{ }^{232} \mathrm{U}, 1$ year old) with $1 \mathrm{~cm}$ of lead shielding. Simulated for a Canberra Falcon 5000 detector $150 \mathrm{~cm}$ from the source, zoomed in on the $1600 \mathrm{keV}$ region. ${ }^{209} \mathrm{Tl}$ is part of the ${ }^{233} \mathrm{U}$ progeny and is the main, if not at times the only, signature in the shielded spectra. 


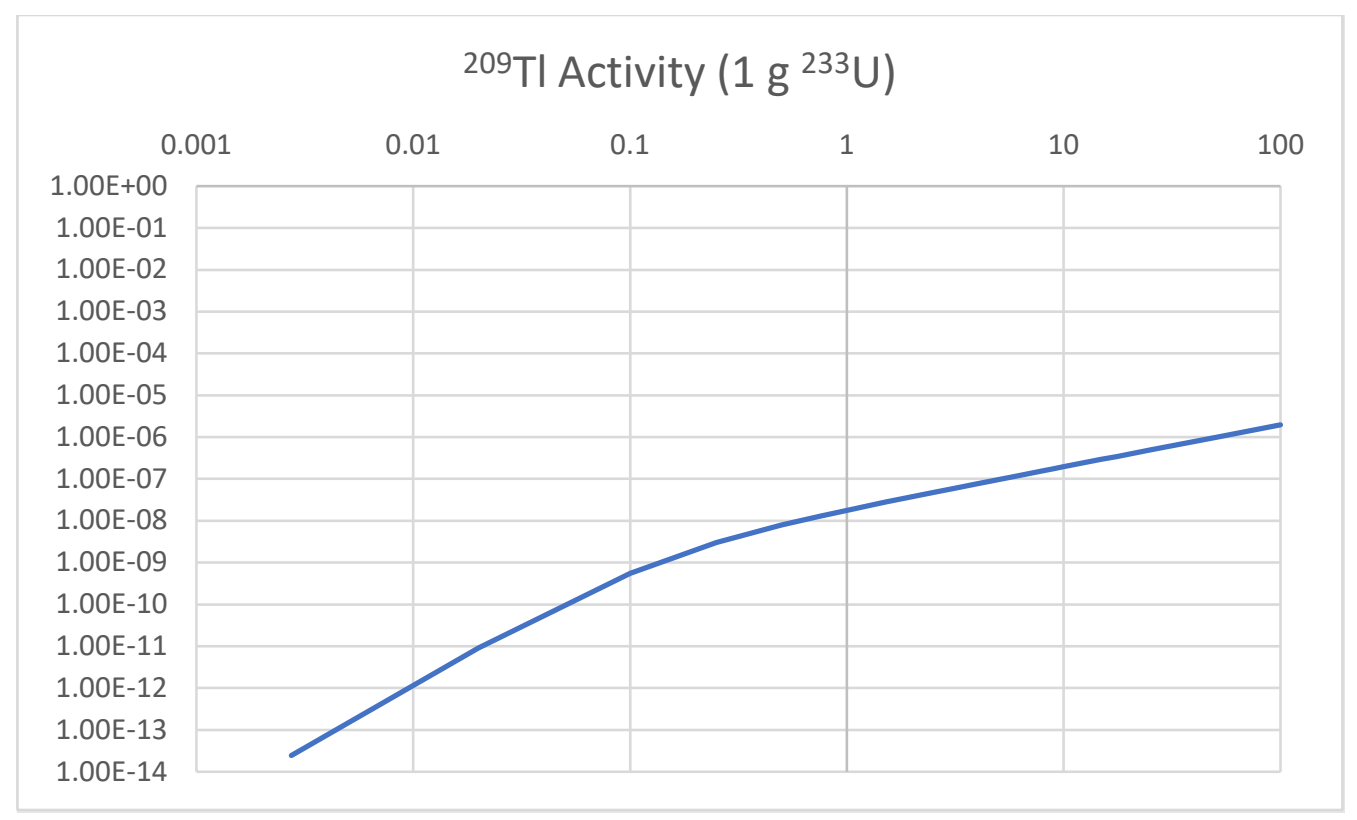

Figure 88. Activity of ${ }^{209} \mathbf{T l}$ in a $\mathbf{1} \mathbf{g}^{233} \mathbf{U}$ item. Because this is the main signature of ${ }^{233} \mathrm{U}$ at higher energies, it is more useful for older items than fresher items. In the United States, ${ }^{233} \mathrm{U}$ items are typically old, on the order of 50 years, and will have significant amounts of ${ }^{209} \mathrm{Tl}$. This simplifies the measurement of even these shielded items, so long as the age is known.

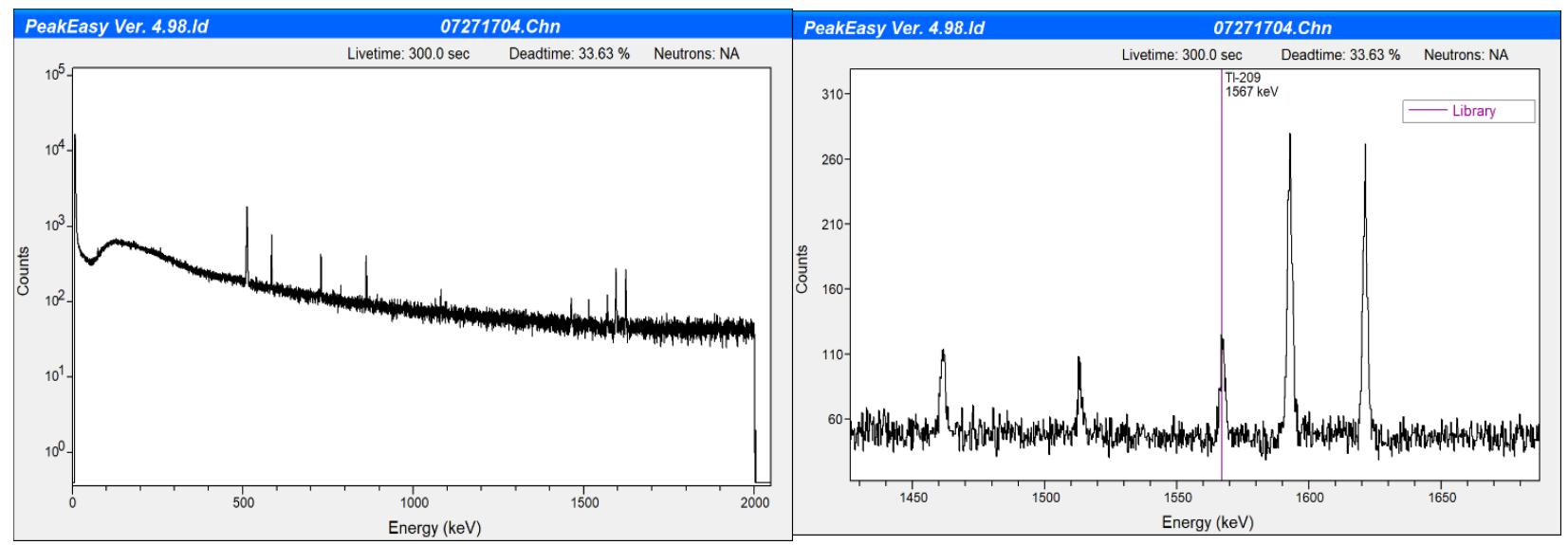

Figure 89. A measured spectrum of an $\sim 150$ gram ${ }^{233} \mathrm{U}$ source with only a few ppm ${ }^{232} \mathrm{U}$ contamination. This item is $~ 50$ years old. In older items such as this, the $1567 \mathrm{keV}$ peak is strong enough to be measured with excellent precision in HPGe spectra.

\subsubsection{Conflicts with Other Gamma Ray Signatures}

Another significant challenge for gamma ray NDA is that, in a thorium fuel cycle, there will be a prevalence of freshly processed thorium materials. When ${ }^{\text {nat }}$ Th is processed for milling and fuel fabrication, both ${ }^{232} \mathrm{Th}$ and ${ }^{228} \mathrm{Th}$ are removed from the rest of the ${ }^{\text {nat }} \mathrm{Th}$ progeny. Within this process, ${ }^{232} \mathrm{Th}$ from ${ }^{228} \mathrm{Th}$ cannot be separated from one another. Notably, however, all the ${ }^{228} \mathrm{Ac}$ is removed, which has significant consequences for the capabilities of many gamma-based NDA techniques and instruments. This is because the ${ }^{232} \mathrm{Th}$ daughter ${ }^{228} \mathrm{Ac}$ gives rise to the only distinguishing decay gamma rays between ${ }^{232} \mathrm{Th}$ and ${ }^{228} \mathrm{Th}$. This is evident from the overlapping decay chains of ${ }^{232} \mathrm{U}$ and the thorium series $\left({ }^{228} \mathrm{Th}\right.$ and ${ }^{232} \mathrm{Th}$ ) shown in Figure 81, which also reveals that the gamma ray signature of ${ }^{232} \mathrm{U}$ is 
essentially the same as for ${ }^{228} \mathrm{Th}$. As a result, freshly processed thorium does not exhibit the distinguishing ${ }^{228} \mathrm{Ac}$ peaks and is also difficult to distinguish from ${ }^{232} \mathrm{U}$ by low- and medium-resolution gamma ray detectors. Two examples are shown in Figure 90 where the lack of ${ }^{228} \mathrm{Ac}$ peaks is apparent, as in Figure 83. Therefore, the presence of ${ }^{228} \mathrm{Ac}$ provides a good indication that the assayed material originates from ${ }^{232} \mathrm{Th}$ and not ${ }^{232} \mathrm{U}$.

While detecting ${ }^{228} \mathrm{Ac}$ peaks is difficult with low-resolution and medium-resolution detectors, ${ }^{228} \mathrm{Ac}$ would be easily detectable if it was instead measured with a HPGe detector. For safeguards applications wherein both recently processed thorium and ${ }^{233} \mathrm{U}$ materials are present and combined within mixtures, high-resolution detectors are generally required. However, even though low- and mid-resolution gamma detectors may not be able to differentiate between ${ }^{232} \mathrm{Th}$ and ${ }^{232} \mathrm{U}$-the latter of which may be an indirect signature of ${ }^{233} \mathrm{U}$-the $2614 \mathrm{keV}$ peak of the ${ }^{232} \mathrm{U}$ daughter ${ }^{208} \mathrm{Tl}$ can still be registered with low-resolution detectors, should it be above natural background levels.
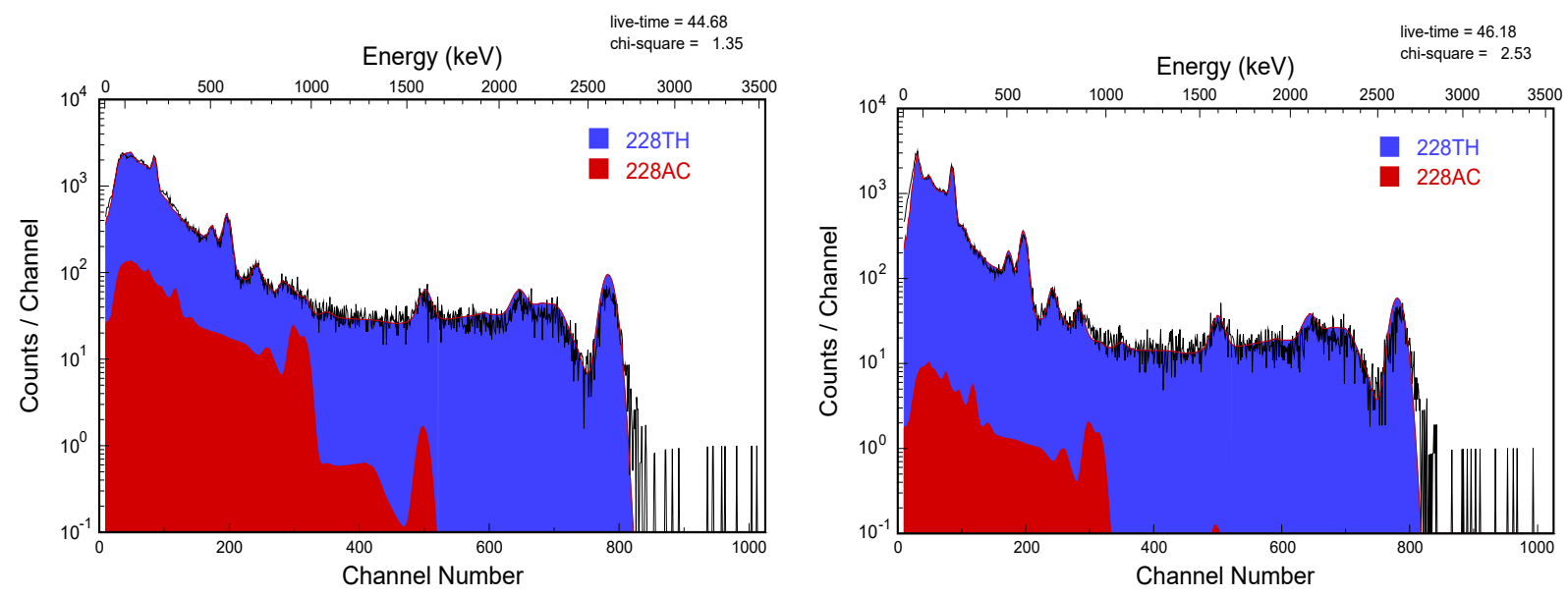

Figure 90. Spectra for freshly processed thorium. Freshly processed thorium does not have strong ${ }^{228}$ Ac peaks at 338,911 , and $969 \mathrm{keV}$. This can cause misidentification as ${ }^{232} \mathrm{U}$, which in turn would imply the presence of ${ }^{233} \mathrm{U}$.

(Left) A shipment of 800,000 thorium lantern mantles; ${ }^{228} \mathrm{Ac}$ at only $20 \%$ of equilibrium activity. (Right) A small container of fresher lantern mantles, with the ${ }^{228} \mathrm{Ac}$ at less than $1 \%$ of the equilibrium activity.

\subsubsection{Software Used}

GADRAS 18.8.1 [46] was used for simulating many of the spectra shown in this report. This software uses a detector characterization and a 1D model of a source as an input for these simulations. There are multiple published evaluations of this simulation capability (e.g., SAND2009-6550, "Benchmarks for GADRAS Performance Validation" by Mattingly et al. [77]). PeakEasy 4.98 .1 was used for plotting and labeling spectra and served as the primary reference of relevant nuclear data.

\subsection{Key Observations and Findings}

\subsubsection{Summary of Gamma NDA Feasibility}

In traditional nuclear safeguards as implemented in U/Pu fuel cycles, gamma-based NDA instruments and methods provide qualitative information that is complementary to the quantitative information provided by neutron-based instruments and methods. Considering the general properties of gamma 
and neutron signatures, it is natural to extend these methodologies to the thorium fuel cycles safeguards as well. However, as we demonstrated in previous sections, gamma-based NDA faces several unique challenges, which suggests the need for further R\&D prior to the adoption of gammabased NDA methods and instruments, which were developed for the U/Pu fuel cycle, within Th/ ${ }^{233} \mathrm{U}$ fuel cycle applications.

One of the greatest challenges stems from the need to take safety precautions against potentially high gamma radiation dose rates resulting from ${ }^{232} \mathrm{U}$ progeny, which in many instances is a natural contaminant of reprocessed ${ }^{233} \mathrm{U}$. This either results in the need to significantly shield the item under assay, hampering the ability to properly measure large parts of the unique signature, or adopt different conduct of operations, where unshielded items could be assayed presumably remotely.

The second most prominent challenge stems from the similarity of gamma signatures for ${ }^{232} \mathrm{Th}$ and ${ }^{232} \mathrm{U}$, as they share a large part of the decay chain. This challenge can, however, be technologically overcome by performing assay measurements with high-resolution spectrometers to identify distinguishing features. That is not infeasible in principle but would require significant modification of conduct of operations and investments into portable/handheld high-resolution technology. While portable, electrically cooled HPGe detectors are commercially available, their reliability and ease of operations cannot yet be compared to compact and rugged handheld detectors such as the identiFINDER HM-5 and other sodium iodide- or cadmium zinc telluride-based devices. Recommendations for future work are provided in Section 5.

\subsection{Destructive Assay in the Context of Thorium Fuel Cycles}

\subsubsection{Overview of destructive assay techniques at the IAEA}

DA techniques are most prominently used by the IAEA for environmental sampling. Note that other methods include uranium enrichment verification and plutonium verification during reprocessing. In conventional U/Pu-based nuclear fuel cycles, these methods are applied to the analysis of cotton swipes used by inspectors to gather environmental samples from nuclear facilities, such as gas enrichment centrifuge plants. DA aims to quantify the isotopic composition of nuclear material contained in the environmental sample. This quantification may confirm declared nuclear activities or provide evidence of past or undeclared nuclear activities. Essentially, the DA results assist the IAEA in evaluating whether a State is compliant with its safeguards obligations.

In keeping with current practice, DA requirements for facilities operating thorium fuel cycles are anticipated to be analyzed through the IAEA's Network of Analytical Laboratories (NWAL). Therefore, the physical nature of the samples is not anticipated to change. The analytes of interest will, however, likely be different for the thorium fuel cycle and require some analytical development work to provide satisfactory results without straining already-limited human and material resources at the IAEA and NWAL.

\subsubsection{Analytes for the Thorium Fuel Cycle}

To generate power from thorium, the nonfissile ${ }^{232} \mathrm{Th}$ isotope (typically combined with a certain amount of fissile uranium and/or plutonium) must be first exposed to a strong neutron flux. The 
resulting ${ }^{233} \mathrm{Th}$ then decays to its daughter isotope, ${ }^{233} \mathrm{~Pa}$, which subsequently decays to fissile ${ }^{233} \mathrm{U}$ (Figure 91). This production mechanism will be the same irrespective of the type of thorium fuel cycle.

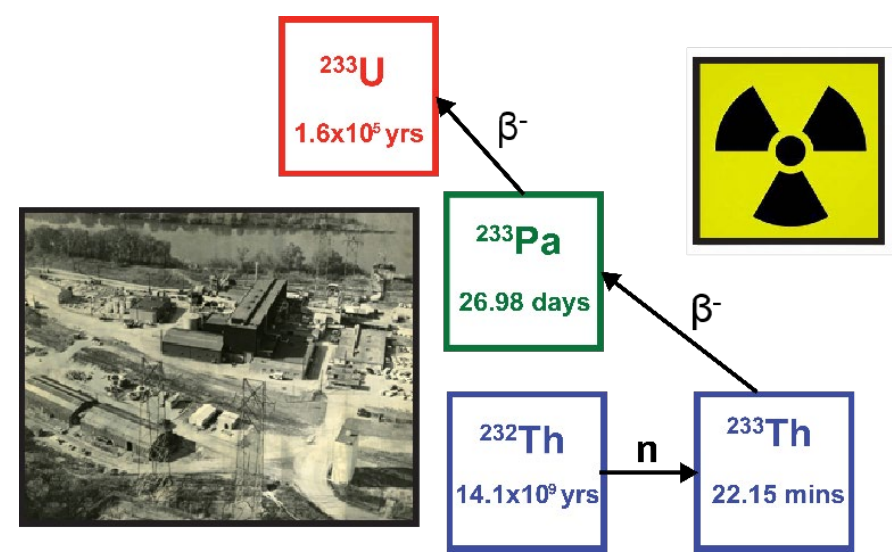

Figure 91. Nuclear transmutation, decay processes, and half-lives of isotopes involved in the thorium fuel cycle. The photo (bottom left) shows the Shippingport Atomic Power Station where the thorium fuel cycle was first demonstrated [78]. See also Figure 79.

The presence of ${ }^{232} \mathrm{U}$-and its intense, highly penetrating gamma-emitting progeny ${ }^{208} \mathrm{Tl}-$ formed by a combination of several different reactions on, and by decays of, ${ }^{232} \mathrm{Th},{ }^{233} \mathrm{Th},{ }^{233} \mathrm{~Pa},{ }^{231} \mathrm{~Pa}$, and ${ }^{233} \mathrm{U}$, results in fresh $\mathrm{Th} /{ }^{233} \mathrm{U}$ fuel being difficult to handle [79]. However, the separation of ${ }^{233} \mathrm{~Pa}$ from the fuel in MSRs could yield ${ }^{233} \mathrm{U}$ relatively free of ${ }^{232} \mathrm{U}$ contamination.

It is likely that the trace analytes of interest in DA of cotton swipes from thorium fuel cycle facilities will include isotopes from the conventional U/Pu nuclear fuel cycle as well as thorium fuel cyclespecific isotopes, including ${ }^{232} \mathrm{Th},{ }^{233} \mathrm{~Pa},{ }^{231} \mathrm{~Pa}$, and ${ }^{233} \mathrm{U}$. These can be measured using existing state-ofthe-art isotope dilution mass spectrometry techniques; however, some modifications will need to be performed for thorium fuel cycle-specific analytes.

As illustrated in Figure 92, Isotope dilution mass spectrometry enables the quantification of the isotopes of interest by:

- adding a solution enriched in an isotope (to almost pure concentrations) that is not to be measured,

- purifying the sample only for the element of interest, and

- measuring the isotopes of interest on a mass spectrometer. 

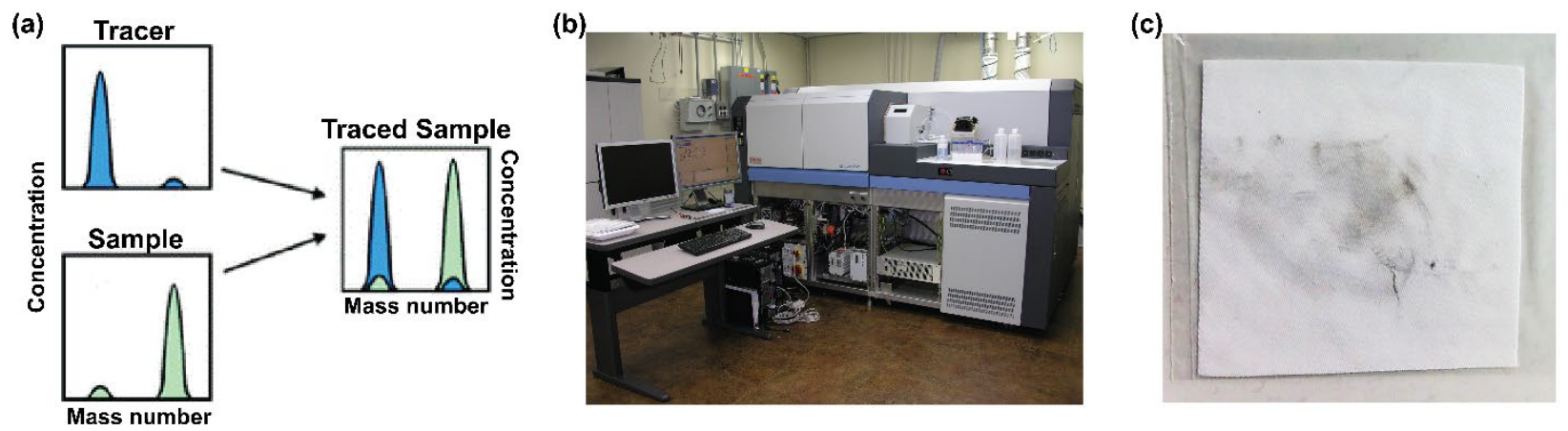

Figure 92. Analytical plan for (a) isotope dilution where blue represents the tracer and green represents sample isotope ratios. If the tracer concentration is accurately known, the sample concentration can be measured by (b) mass spectrometry. Sample testing could involve doping cotton swipes (c).

The tracer is a solution enriched in an isotope, preferably only the isotope being measured. Ideally, for quality assurance purposes, this will be a certified reference material (e.g., ${ }^{229}$ Th Nuclear Forensics Reference Material [80]); However, well-characterized in-house materials (e.g., LANL Delta-17 ${ }^{233} \mathrm{U}$ tracer) can suffice. The tracer is added and equilibrated with the sample prior to chemical separation. Because the tracer is the same element, but a different isotope from the analytes of interest, it will behave similarly during chemical separation; therefore, the resulting solution for mass spectrometry analysis will be the isotope(s) of interest plus the added tracer isotope. If the tracer solution contains significant quantities of the isotopes of interest, the measurement uncertainty will increase. Thus, it is important that the tracer solution is as pure as possible.

It may, however, become challenging to properly quantify isotopic composition of samples that already contain significant amounts of isotopes that would be otherwise used as tracers. For example, if the cotton swipe bears small but detectable levels of all uranium isotopes, from ${ }^{232} \mathrm{U}$ to ${ }^{238} \mathrm{U}$, the relative isotopic composition could be determined in a two-step process in which the sample solution is split into two subsamples and each is processed with a different tracer. Such a method would likely not increase the complexity of the task significantly, but simply would double the effort required for processing the original sample solution. In any case, obtaining the correct tracers at appropriate purity will likely be one of the greatest challenges to carrying out DA measurements of samples from a facility's thorium fuel cycle. Moreover, the radiation levels of some samples may require additional infrastructure to protect the analysts. The following sections provide a summary of the status and challenges for the isotope dilution measurement for each potential analyte of interest for the Th fuel cycle.

\subsubsection{1 ${ }^{233} \mathrm{U}$ analytes}

$\mathrm{A}^{233} \mathrm{U}$ tracer is commonly used for uranium measurements of samples from the U/Pu nuclear fuel cycle. This tracer would not be appropriate to use in the measurement of samples when ${ }^{233} \mathrm{U}$ is the isotope of interest. A new tracer would be required, most likely ${ }^{236} \mathrm{U}$, which is rarely found in nature. The ${ }^{236} \mathrm{U}$ tracer would need to be produced or characterized in house. In general, uranium purifications are not technically challenging, although, logistically, separate laboratories from those used to process U/Pu fuel cycle samples would be needed to avoid cross-contamination between the different tracers. In addition, the potential high radiation levels may require different analytical laboratory infrastructure from that required for conventional DA such as lead or tungsten shielding, which in turn 
may lead to contamination of the environment and samples with these heavy elements. We recommend that future work include assessing the impact of these infrastructure modifications on the chemical purifications and/or mass spectrometry.

\subsubsection{2 ${ }^{232}$ Th analytes}

Chemical separation and mass spectrometry methods are straightforward for the analysis of ${ }^{232} \mathrm{Th}$. The ${ }^{229} \mathrm{Th}$ tracer routinely used for thorium measurements would also be appropriate for samples from the thorium fuel cycle. Any samples with high radiation levels may require certain analytical laboratory infrastructure modifications such as shielding, which may potentially contaminate the samples. In addition, it can be challenging to maintain a low ${ }^{232} \mathrm{Th}$ blank due to the widespread occurrence of ${ }^{232} \mathrm{Th}$ in nature. An assessment of thorium blank levels prior to starting measurements would likely be required.

\subsubsection{3 ${ }^{231} \mathrm{~Pa}$ and ${ }^{233} \mathrm{~Pa}$ analytes}

It may be challenging to identify a tracer that can be used for both ${ }^{231} \mathrm{~Pa}$ and ${ }^{233} \mathrm{~Pa}$ measurements. Depending on the respective concentrations of both ${ }^{231} \mathrm{~Pa}$ and ${ }^{233} \mathrm{~Pa}$, it may be possible to assume negligible concentrations of ${ }^{231} \mathrm{~Pa}$ produced from $(n, 2 n)$ reactions on ${ }^{232} \mathrm{Th}$ and to use existing ${ }^{231} \mathrm{~Pa}$ standards or in-house ${ }^{231} \mathrm{~Pa}$ solutions as the tracer. Alternatively, ${ }^{231} \mathrm{~Pa}$ may be measurable by another NDA method. Chemical separation and mass spectrometry methods for Pa are well established. However, for mass spectrometry it will be important to consider the need for measurement immediately after the final chemical separation because ${ }^{233} \mathrm{~Pa}$ decays to ${ }^{233} \mathrm{U}$, which will interfere with the ${ }^{233} \mathrm{~Pa}$ measurement as it has the same mass. The short half-life of ${ }^{233} \mathrm{~Pa}$ may also make the logistics of DA measurements challenging. We recommend that future work include a feasibility study to ascertain whether the current distribution system of cotton swipes by the IAEA to NWAL is fast enough to allow accurate ${ }^{233} \mathrm{~Pa}$ measurements before significant radioactive decay has occurred. This feasibility study could be performed using a cotton swipe doped with ${ }^{233} \mathrm{~Pa}$ (e.g., Figure 92(c)).

\subsubsection{Summary of DA Feasibility}

While it appears that safeguards technology required for DA of samples from thorium fuel cycles is essentially the same as for samples from U/Pu fuel cycles, and therefore is readily available, the complexity of the analysis and associated logistics gives rise to some potential challenges. For example, should environmental samples contain high radiation levels complicating their safe handling, the need for shielding may lead to potential contamination and interferences by traces of the shielding material itself. The problem of contamination in general is compounded if multiple tracers are needed for characterization of the same sample. Use of multiple tracers may lead to increased demand on infrastructure to separate analytical processes. Furthermore, the logistics of environmental sample distribution, handling, and prompt processing to accommodate DA of fastdecaying protactinium isotopes is unprecedented in U/Pu fuel cycles. While this by itself is perhaps not an unsurmountable obstacle (pending confirmation by a dedicated feasibility study), backlogs have been encountered in the processing of current environmental samples, which raises the challenge of accommodating this new stream of thorium fuel cycle samples. Perhaps on-site laboratories will be required for DA methods for protactinium. 


\section{Overview of the Legal Framework for Implementation of International Nuclear Safeguards and Safeguards Technology in the Thorium Fuel Cycle}

This section describes the legal framework for international nuclear safeguards implementation through the IAEA. The existing legal framework includes fertile ${ }^{232} \mathrm{Th}$ and fissile ${ }^{233} \mathrm{U}$ within the definition of nuclear material that is subject to safeguards; however, most commercial nuclear facilities currently under safeguards rely on the $\mathrm{U} / \mathrm{Pu}$ (primarily ${ }^{235} \mathrm{U}$ and ${ }^{238} \mathrm{U}$ ) fuel cycle. As a result, international nuclear safeguards concepts and approaches, as well as technologies, have focused nearly exclusively on the U/Pu fuel cycle. This section should not be considered a fully comprehensive legal analysis of key safeguards-relevant documents and treaties, but rather as a high-level examination of whether and how the current legal safeguards framework might guide (or at times dictate) future implementation of international nuclear safeguards in the thorium fuel cycle. The main objective of this section is therefore to identify how this legal framework impacts the implementation of safeguards technology and thus the potential safeguards technology R\&D needs that are the focus of this report.

\subsection{History of the IAEA}

The IAEA resulted from President Dwight D. Eisenhower's "Atoms for Peace" speech, given to the United Nations General Assembly in 1953. Eisenhower proposed that the United Nations create an international organization that would support safe and peaceful use of nuclear energy while also verifying that nuclear technology is not misused for nuclear weapon development. As a result, the Statute of the IAEA entered into force in 1957, forming the legal basis for the establishment and implementation of international nuclear safeguards. Over time, this legal framework has evolved, such as with the entry into force of the NPT in 1970 [81].

\subsection{Legal Framework for International Nuclear Safeguards}

This section provides a summary ${ }^{3}$ of key legal instruments.

\subsubsection{Statute of the IAEA}

According to the Statute of the IAEA [82]:

\footnotetext{
${ }^{3}$ The legal framework for international nuclear safeguards is discussed in this report roughly in chronological order. Information relevant to this report is summarized mostly from Ref. [81], which is much more comprehensive than the discussion here; for a full overview of the legal framework, please see Ref. [81]. When information is derived from additional sources, it is indicated.
} 
- The IAEA is authorized to establish, administer, and apply safeguards to any of the nuclear activities of a State, at that State's request (Article III.A.5).

- The IAEA (and its staff of inspectors) has the right and responsibility to verify compliance by (Article XII):

○ examining the design of specialized equipment and facilities, ${ }^{4}$

- examining operating records and reports pertaining to material accounting and control, and

- conducting in-person inspections of places and data dealing with materials, equipment, or facilities subject to safeguards.

\subsubsection{Nonproliferation Treaty}

- The Statute of the IAEA requires each State party to the NPT to accept safeguards, via an agreement with the IAEA, on all source or special fissionable material ${ }^{5}$ in all peaceful nuclear activities within its territory, under its jurisdiction, or carried out under its control anywhere (Article III.1).

\subsubsection{Regional Treaties}

Several regional treaties, such as those creating nuclear-weapon-free zones (e.g., Tlatelolco Treaty) or bilateral inspectorates (e.g., the Brazilian-Argentine Agreement), have put in place several regional requirements. As they are not universal in nature and serve to reinforce the IAEA and the international nuclear safeguards framework, rather than contributing to the legal framework, they are not discussed further here.

\subsubsection{Safeguards Documents}

\subsubsection{INFCIRC/66/Rev.2}

INFCIRC/66 agreements (also known as item-specific agreements) are in place today in India, Pakistan, and Israel. These agreements obligate a State to legally binding safeguards on a specific nuclear facility (or facilities) but do not cover nuclear material and facilities in the State as a whole. INFCIRC/66-type agreements require the State to:

- Notify the IAEA of receipt of safeguarded items (i.e., nuclear material, nonnuclear material, facilities, equipment, or components), and

\footnotetext{
${ }^{4} \mathrm{~A}$ facility is defined in INFCIRC/66 as "a reactor, a reprocessing plant, an isotope separation plant, a conversion plant, a separate storage installation, and a fuel fabrication plant (not to include a mine or ore-processing plant)." Additional facilities are defined in INFCIRC/153 and INFCIRC/540, including a critical facility or "any location where nuclear material in amounts greater than one effective kilogram is customarily used" $[6,8,83$, 84].

${ }^{5}$ Nuclear material is defined to be source and special fissionable material (i.e., DU, natural uranium, thorium, ${ }^{239} \mathrm{Pu}$, ${ }^{233} \mathrm{U}$, uranium enriched in the isotopes 235 or 233 ) $[8,83]$.
} 
- Maintain accountancy of any nuclear material produced, processed, or used in safeguarded items.

\subsubsection{INFCIRC/153/Corr.}

INFCIRC/153 is the model safeguards agreement for what are commonly referred to as "full scope" or "comprehensive" safeguards agreements. As of 2018, 174 States have CSAs with the IAEA [6]. Upon entry into force, the State must:

- Submit an initial report to the IAEA of all nuclear material in the State, and

- Submit a list of all nuclear facilities in the State (including those without nuclear material currently present or those under construction), including design information.

This information allows the IAEA to verify declarations as well as conduct design information verification (DIV).

\subsubsection{INFCIRC/540/Corr.}

INFCIRC/540 is the "Model Protocol Additional to the Agreement(s) Between State(s) and the International Atomic Energy Agency for the Application of Safeguards," commonly referred to as the Additional Protocol or AP. As of 2018, APs have been concluded by 134 States with the IAEA in addition to a CSA [86]. These agreements provide the IAEA with additional rights and State information, including:

- Information about all aspects of a State's nuclear fuel cycle, from uranium mines to nuclear waste,

- Information about all buildings on a nuclear site,

- Information about fuel cycle-related R\&D,

- Information about the manufacture and export of sensitive nuclear-related technologies, and

- Collection of environmental samples beyond declared locations, when deemed necessary by the IAEA.

INFCIRC/540/Corr. allows for CA to locations for which the State has not declared the presence of nuclear material or nuclear facilities; $C A$ is in addition to on-site inspections and DIVs allowed under INFCIRC/153/Corr.

\subsubsection{Small Quantities Protocol (GOV/INF/276, Annex B)}

Like the AP, the Small Quantities Protocol is put into effect along with a CSA and is intended for States with little or no nuclear material and/or activities in their territories (e.g., a State with only nuclear cancer treatment technology). The SQP would likely not be relevant for a State with a thorium fuel cycle, however, and thus is not discussed further here. 


\subsubsection{Voluntary Offer Agreements}

The NPT defines five NWSs: the United States, the UK, France, China, and Russia (formerly, the USSR). All other States party to the NPT are NNWSs. As the five NWSs already have nuclear weapons, and military fuel cycles are not subject to international nuclear safeguards, these five States conclude voluntary offer agreements (VOAs) with the IAEA. These are provided within the framework of CSAs under INFCIRC/153/Corr. An NWS provides a list of commercial facilities from which the IAEA may select for inspections. The IAEA often selects novel or unique facilities on the list that allow the IAEA to test new safeguards measures, which they may then choose to employ more broadly. If an NWS places a thorium fuel cycle facility under their VOA, the IAEA may preferentially select it. All NWSs have an AP in force.

\subsection{Application to the Thorium Fuel Cycle}

Several legal instruments dating back to the 1950s form the framework for the application of international nuclear safeguards. Key takeaways for the thorium fuel cycle are as follows:

- $\quad{ }^{232} \mathrm{Th}$ and ${ }^{233} \mathrm{U}$ are defined as source material and special fissionable material, respectively, dating back to the IAEA Statute. As such, both are defined by the IAEA as nuclear material, and thorium fuel cycle materials are subject to international nuclear safeguards.

- A facility is defined, dating back to the 1960 s, to include a wide range of operations, including a nuclear reactor, fuel conversion/fabrication plants, and reprocessing plants. Thorium fuel cycle facilities clearly meet the existing definition of a facility and, thus, are subject to international nuclear safeguards.

- IAEA safeguards have been implemented in thorium fuel cycles in the past and on a continuing basis $[16,86]$. The IAEA Annual Report's "Additional Annex Information" provides a list of facilities under safeguards for each NNWS. In the 2018 Annual Report, thorium fuel cycle facilities under safeguards include:

- THTR-300 (Germany; thorium high-temperature gas reactor, operated 1985-1989),

- KWL-60 (Germany; BWR with test Th-Pu oxide fuel, operated until 1973), and

- NRU/NRX (Canada; research reactors with irradiation tests on Th- ${ }^{235} \mathrm{U}$ fuel materials, currently in operation).

However, it is unclear what types of safeguards measures were/are implemented by the IAEA in these facilities.

- An NWS (e.g., the United States, China) developing a commercial thorium fuel cycle may opt to list a thorium-based facility under their VOA with the IAEA. If so, the IAEA could select it for inspections, potentially for the purpose of developing new safeguards implementation procedures or measures. Additionally, an NWS could sell their thorium fuel cycle technology to an NNWS with a CSA, and those reactors and associated nuclear materials would be subject to IAEA safeguards in that NNWS, even if not originally placed under the originating NWS's VOA. 
- Thorium fuel cycle facilities can optionally be placed under safeguards through an item-specific agreement. For example, all civilian nuclear fuel cycle facilities in India are currently subject to IAEA safeguards. India's PFBR (a thorium-blanket breeder reactor) and KAMINI ( ${ }^{233} \mathrm{U}$-fueled) reactor have not been declared as commercial nuclear facilities and thus are not subject to IAEA safeguards; however, if these reactors move from the R\&D/pilot-scale phase to electricity production, they may become subject to IAEA safeguards if India opts to place them under safeguards. Additionally, as with China, if India chooses to commercialize its thorium-based reactors and sells them to an NNWS with a CSA, those reactors and associated nuclear materials would be subject to IAEA safeguards.

- If a State has a CSA (with or without an AP), it must declare a thorium fuel cycle facility as soon as plans for construction have been made, whether it is a domestic design or a design to be purchased from another State. For example, Indonesia is exploring the purchase of a thorium MSR from ThorCon, a US company; because Indonesia has a CSA in place with the IAEA, such a reactor (and all associated nuclear material) would be subject to safeguards in Indonesia.

- As with the U/Pu fuel cycle, the State must provide facility design drawings and material accountancy declarations for the thorium fuel cycle throughout its lifecycle.

- The IAEA must independently verify a State's declarations to confirm all material and facilities subject to safeguards remain in peaceful use. Most of the IAEA's verification technologies are developed with the U/Pu fuel cycle in mind. It is unclear whether existing IAEA verification technologies are effective in verifying the thorium fuel cycle. If they are found to be lacking, the IAEA may require modified or new technologies to mitigate gaps.

\subsection{Complementary Access in the Context of Thorium Fuel Cycles}

\subsubsection{Overview of Complementary Access under the Additional Protocol}

The "Model Protocol Additional to the Agreement(s) between State(s) and the IAEA for the Application of Safeguards," referred to as the AP, affords the IAEA additional rights to those provided in a CSA, VOA, or item-specific agreement. At a high level, the AP allows the IAEA access to additional types of nuclear fuel cycle facilities (e.g., uranium mining facilities, R\&D facilities), including those that do not normally store or use nuclear material, as well as undeclared locations within the State. ${ }^{6}$ The AP also requires a State to make expanded declarations, and it includes language to streamline access for inspectors. CA is applicable to all States for which the AP already entered into force [81].

For states with an AP in force, CA allows the IAEA to verify the absence of undeclared nuclear material and activities. (Design information verification and on-site inspection activities are intended to verify no diversion of declared nuclear material or facility misuse for the expanded State declarations.) This means that the CA inspection equipment used should be able to identify attributes of potential proliferation indicators (PIs).

According to Article 5 of the AP [83], CA can be conducted in three types of locations:

\footnotetext{
${ }^{6}$ India is an exception. It holds an item-specific agreement rather than a CSA.
} 
1. At facilities declared under a CSA, but at additional locations within the declared site that are specified by the AP (e.g., locations holding nuclear material for which safeguards has been terminated),

2. At additional facility types declared under an AP (e.g., uranium mining facilities), and

3. Any other location within the State specified by the IAEA.

It should be noted that IAEA rights are more limited with respect to CA: only environmental sampling is allowed, unless it does not resolve the discrepancy, in which case, visual observation, radiation detection, and other measurements devices could be allowed at a future CA visit.

\subsubsection{Complementary Access Toolkit}

Such unique objectives for CA, discussed in Section 4.4.1, inherently call for reduced numbers (or a subset) and specific types of instruments to be used during CA different from those used during onsite inspections under a CSA. Safeguards equipment for on-site inspections tends to be specific to the facility type (e.g., HPGe detectors at enrichment plants; Cherenkov Viewing Device at reactors). These pieces of equipment are bulky, so they either reside at the facility or the regional office or are shipped from Vienna to the facility, whereas the CA kit is hand-carried by the inspectors. As the safeguards activities and related tools for the various nuclear facilities differ, specialized kits are not available as for CA. In the case of CA, the activities are more standard (assuring the absence of undeclared nuclear material and activities, as opposed to verifying no diversion of declared nuclear material or facility misuse). The instruments employed for CA have been traditionally organized in a CA Toolkit, which until recently comprised:

- A digital camera,

- A laser distance meter,

- A GPS tool,

- A voice recorder,

- A flashlight,

- A general-purpose radiation measurement system (typically an identiFINDER HM-5 gamma detector), and

- An environmental sampling kit.

From 2013 until around 2016, significant effort was devoted to properly cataloguing nuclear or other material, activities, processes, and instrumentation that could correlate with undeclared fuel cycle activities [86] and to identifying COTS equipment that could measure or identify these materials [87]. These studies were primarily based on the IAEA's Physical Model, which exist in volumes, with each volume pertaining to a given facility type. One of the major findings was that the original CA Toolkit was not well-suited for identification or measurement of many potential PIs and needed to be expanded with additional, preferably handheld, instruments that allow elemental and chemical 
identification during nuclear material accounting activities [88]. Since then, the IAEA performed its own evaluations and has gradually introduced novel or upgraded instrumentation into the CA Toolkit. In Ref. [89], the new toolkit is referred to as the Multi Components Inspectors Kit (MCIK). In the same document, it is also claimed that MCIK has already fully superseded the original CA Toolkit. Although at the time this report was written, the IAEA was still pursuing authorization for use and inclusion of chemical spectrometers into the MCIK, the types of handheld instruments that will become available for CAs in the near-and midterm future are likely to include $x$-ray fluorescence spectrometers capable of metal alloy elemental traces identification, Fourier-transform infrared spectrometers, and Raman spectrometers that can detect various nonradioactive chemical species used within the nuclear fuel cycle; laser breakdown spectrometers, in principle capable of identifying various chemical forms of actinides; and gamma-imaging cameras. The conduct of operations, including individual instruments and potential in-field scenarios, will likely be a subject of continuous learning and implementation effort; however, the true potential for CA for thorium fuel cycles is governed by individual detector technology capabilities in their handheld form.

From the perspective of thorium fuel cycle safeguards under an AP (i.e., CA of a thorium fuel cycle facility), the objective remains the same: make a determination as to whether undeclared activities are occurring in the State to allow drawing a broader conclusion for the State for the calendar year. The CA is not intended to fully quantify or characterize "newly discovered" undeclared activities or material. That would likely be the goal of any follow-up inspections and other actions that the IAEA could take regarding potential noncompliance. Thus, evaluation of the suitability of the existing CA Toolkit-or the new (and still evolving) MCIK-starts with understanding what the potential PIs are.

\subsubsection{Physical Model and Proliferation Indicators}

Many of the potential PIs are known or can be safely assumed based on the IAEA Physical Model, which includes certain generic types of thorium-based fuel cycles. Moreover, nuclear material in its isotopic or chemical forms typical of any, or most relevant, thorium fuel cycle concepts are also known. Considering the nature of gamma and neutron signatures, in the majority of cases, it is the gamma signatures that reveal the attributes of the nuclear material because the $\mathrm{SF}$ of ${ }^{232} \mathrm{Th}$ or ${ }^{233} \mathrm{U}$ is too low for any practical neutron-based detection methods. Given the detector energy resolution of currently used handheld gamma instruments in the CA Toolkit (e.g., identiFINDER HM-5), this may still not be enough to unambiguously identify a potential PI (e.g., presence of undeclared fissile material) for ${ }^{233} \mathrm{U}$; however, it should be enough to prompt additional scrutiny. Recalling the findings on gamma signatures in Section 3.4, low- and mid-resolution gamma detectors may not be able to differentiate between ${ }^{232} \mathrm{Th}$ and ${ }^{232} \mathrm{U}$, the latter of which may be an indirect signature of ${ }^{233} \mathrm{U}$. However, even the lowest-resolution detectors can register the 2614 keV peak of ${ }^{208} \mathrm{Tl}$ should it be above natural background levels, pointing to the presence of thorium fuel cycle-related material. Overall, it appears signatures of nuclear material from thorium fuel cycles could be detected during CA with current instruments, or instruments deployed in the near future from the MCIK. Early detection by the IAEA of proliferation activities is desirable. Considering detection of traces of nuclear material that may indicate pilot phases of a more sophisticated program, CA will (even in the case of thorium fuel cycles) rely primarily on environmental sampling, currently performed with $100 \%$ natural cotton swipes.

Given the general efficacy of cotton swipes to capture a range of materials from different surfaces, it is anticipated that the general removal rates and conduct of operations for thorium fuel cycle facilities will remain similar to those currently performed in existing $\mathrm{U} / \mathrm{Pu}$ fuel cycle facilities. However, 
processing of environmental samples may bring its own challenges, as already discussed (see Section 3.5).

Additionally, the early detection of proliferation activities may be achieved through discovery of nonradiological PIs including nuclear material processing equipment and various chemicals or materials that may or may not be unique to thorium fuel cycles but may serve as surrogates for development of relevant chemical processes, such as uranium or thorium reprocessing. However, because many such PIs are dual-purpose materials or instruments, finding these may not lead directly to high-confidence conclusions. This is a general problem facing CA in conventional U/Pu fuel cycle facilities and is not limited to thorium fuel cycles, but it appears to be addressed by gradual improvement and expansion of the tools available for CA. Gains in the technological capabilities of the verification equipment in conventional U/Pu fuel cycles will likely translate into similar technological gains for thorium fuel cycles as well.

It should be noted that the ability to recognize PIs stems from knowledge of the relevant nuclear fuel cycle and chemical, radiological, or technological processes involved. From that perspective, the entire nuclear safeguards community has accumulated enormous experience with a wide range of facility types across conventional U/Pu fuel cycles. The experience base with thorium fuel cycles remains to be effectively established, and current safeguards considerations (including within this report) based on current concepts may subsequently evolve and significantly differ from actual industrial-scale solutions. While it may take decades for thorium fuel cycle facilities to become commonplace, the safeguards community must keep pace with this development in the industry and be prepared to carry out thorough evaluations of commercial thorium fuel cycle facilities. This will require a dedicated effort to ensure key technological documents reflect evolving technology, some of which may not yet have been fully envisioned. For example, the Physical Model may require revision or amendment of its thorium fuel cycle-related sections, just as Appendix II of the AP (INFCIRC540) [84] may need to be assessed for completeness with respect to emerging technologies and export control status. An export control discussion is provided in Ref. [48].

Overall, the legislative and technological framework of the CA as established and developed for U/Pu fuel cycles remains relevant for Th fuel cycles and provides an efficient and effective template for implementation. Its continued efficiency and effectiveness, however, will depend on our ability to stay in close contact with the progress of current nuclear technology and Th fuel cycle commercialization or further development.

\subsection{Summary}

The legal framework exists for international nuclear safeguards to be implemented for thorium fuel cycle facilities and materials. As advanced reactor designs near commercial deployment, existing IAEA safeguards approaches and verification technologies must be assessed for whether they can be effectively leveraged for novel fuel cycle facilities and nuclear material types the IAEA is less accustomed to verifying. 


\section{R\&D Needs for a Thorium Fuel Cycle Safeguards Technology Development Effort}

Thorium fuel cycles are complex and have unique safeguards challenges, which lead to unique safeguards technology needs. In this scoping study, three leading thorium fuel cycles have been chosen to identify nuclear material types and forms for future verification. They illustrate that thorium fuel cycles involve a greater diversity of nuclear material types and forms than conventional U/Pu fuel cycles. From a safeguards standpoint, it is important to highlight that ${ }^{232} \mathrm{Th}$ and ${ }^{233} \mathrm{U}$ are usually present with other nuclear material in items and bulk material in thorium fuel cycle facilities under safeguards. Therefore, accurately quantifying nuclear material of each type (e.g., mass of ${ }^{232} \mathrm{Th}$ vs. ${ }^{233} \mathrm{U}$ vs. ${ }^{235} \mathrm{U}$ ) usually requires distinguishing between at least two fertile and/or fissile isotopes of uranium and thorium. Thorium fuels utilize a fissile driver (e.g., ${ }^{235} \mathrm{U},{ }^{239} \mathrm{Pu}$ ); therefore, these initial fissile isotopes will be present within fuel cycle items in addition to the ${ }^{233} \mathrm{U}$ that may be present within these fuels, whether in solid or molten salt form. Thus, there is a need to develop verification techniques that can distinguish ${ }^{233} \mathrm{U}$ from other fissile isotopes as well as confirm the presence of fertile ${ }^{232} \mathrm{Th}$ accompanying the fissile driver. This is especially relevant at MSR facilities, where the quantities of each nuclear material type (i.e., ${ }^{233} \mathrm{U},{ }^{235} \mathrm{U}, \mathrm{Pu}$ ) could vary dramatically over the lifetime of a core, especially considering a design that is refueled online and where ${ }^{233} \mathrm{U}$ is continually recycled at the facility. Within the thorium MSR fuel cycle, these measurements may also be performed in the presence of protactinium and its fertile isotopes (e.g., ${ }^{232} \mathrm{~Pa}$ ).

Given the nuclear material types and forms anticipated to be under safeguards, this scoping study has then assessed the most prominent safeguards technology needs for these three leading thorium fuel cycles and posed potential solutions to meet these needs. Currently, safeguards are applied to conventional U/Pu fuel cycles, thus existing safeguards verification technologies are tailored toward ${ }^{235} \mathrm{U},{ }^{238} \mathrm{U}$, and ${ }^{239} \mathrm{Pu}$ detection, identification, characterization, quantification, and monitoring. In this scoping study, many of the traditional safeguards instruments and analysis methods have been shown to be ineffective as designed and implemented for the assay of thorium fuel cycle isotopes, and thus R\&D is needed before they can be effectively applied to thorium fuel cycles. Drawing analogies among neutron signatures and neutron NDA applications for isotopes based on their fundamental nuclear properties and use may spur method development. Specifically, nuclear physics analogies for detection exist between ${ }^{232} \mathrm{Th}$ and ${ }^{238} \mathrm{U}$ and between ${ }^{233} \mathrm{U}$ and ${ }^{235} \mathrm{U}$. Key verification issues include isotopic mixtures and complex items, the low-intensity gamma rays associated with ${ }^{233} \mathrm{U}$, the presence of ${ }^{232} \mathrm{U}$ progeny, shielded materials, a reduction in the current capabilities of passive neutron assay, and the need for active neutron interrogation and development of self-interrogation neutron techniques.

Based on these findings, R\&D needs and recommendations from this scoping study for a thorium fuel cycle safeguards technology development effort are summarized in the tables below. R\&D needs are organized by TRL, the capabilities of current instruments selected for evaluation in this scoping study, the environment in which the measurement would need to be made, and the R\&D needed to achieve the relevant technical safeguards objectives. Thorium fuel cycle safeguards are an emerging issue, but as scientists and safeguards professionals and practitioners, we have the obligation and opportunity to solve these verification challenges for the continued future peaceful use of nuclear material. 


\subsection{R\&D Needs-Lower TRL (1-4) Concept Development and Laboratory Demonstration}

The following recommendations are categorized as lower TRL (1-4), where technology R\&D activities are needed. These activities include the development of fundamental measurement concepts, radiation signature correlations, analysis methods, and prototype instruments and technologies. Other development activities include laboratory demonstration of the developed prototypes. This lower TRL also applies to specific recommendations for the significant modification of new or existing safeguards technologies (both instruments and methods) for application to thorium fuel cycle safeguards. Furthermore, infrastructure needs, such as assembling relevant thorium fuel cycle materials for experimental validation, have been categorized here as lower TRL.

\section{Materials and Infrastructure Needs for Experimental Validation}

- Assemble a representative set of sample materials for experimental evaluation. Materials relevant to the three leading thorium fuel cycles are identified in Sections 1.2.6 and 1.3.1. These supplies should be maintained across the DOE complex to support R\&D and technology development to strengthen verification capabilities.

- Leverage nuclear materials from other scientific communities within the DOE complex, including the nuclear forensics community and the DOE Isotope Program.

Concept Development and Laboratory Demonstration for Neutron NDA of ${ }^{232}$ Th and ${ }^{233} \mathrm{U}$

- Draw analogies between current and anticipated safeguards technologies and methods for concept development based on similarities among ${ }^{233} \mathrm{U}$ vs. ${ }^{239} \mathrm{Pu},{ }^{233} \mathrm{U}$ vs. ${ }^{235} \mathrm{U}$, and ${ }^{232} \mathrm{Th}$ vs. ${ }^{238} \mathrm{U}$.

- Verify that current neutron detectors can work with shielded and bare ${ }^{233} \mathrm{U}$ in different chemical forms (e.g., oxide, fluoride salt).

- Address the safeguards measurement challenge of the quantitative assay of ${ }^{23} \mathrm{U}$ total mass by modifying or developing active neutron NDA techniques because the passive neutron NDA of ${ }^{233} \mathrm{U}$ is not possible. This is not possible because ${ }^{233} \mathrm{U}$ does not have a high enough SF neutron yield and, therefore, does not have a practically usable passive neutron signature.

- Evaluate neutron NDA techniques used for ${ }^{235} \mathrm{U}$ for their applicability to ${ }^{233} \mathrm{U}$.

- Develop active neutron interrogation methods and corresponding analysis algorithms for the quantitative mass assay of ${ }^{233} \mathrm{U}$. Adapting the standard active interrogation technique using ${ }^{241} \mathrm{AmLi}(\alpha, n)$ neutron interrogation sources, currently used for the mass assay of LEU and HEU, provides a starting point.

- Develop and demonstrate a self-interrogation neutron NDA method for pure ${ }^{233} \mathrm{U}$ based on its high $(\alpha, n)$ neutron yield. 
Concept Development and Laboratory Demonstration for Neutron NDA of ${ }^{232} \mathrm{Th}$ and ${ }^{233} \mathrm{U}$

- Develop self-interrogation neutron NDA techniques for which ${ }^{233} \mathrm{U}$ is in the presence of other fissile isotopes and present in an oxide or fluoride compound (not metal). The driver will be ( $\alpha, n)$ reactions on surrounding light elements.

- Demonstrate that ${ }^{233} \mathrm{U}$ can be discriminated from ${ }^{235} \mathrm{U}$ by neutron methods based on selfinterrogation.

- Evaluate active neutron NDA methods for the quantitative assay of ${ }^{232}$ Th total mass, as passive neutron NDA is not possible. The SF rate of ${ }^{232} \mathrm{Th}$ is low (i.e., $1.02 \times 10^{-7}$ neutrons $/ \mathrm{g} / \mathrm{s}$ ). Selfinterrogation is also unlikely because ${ }^{232} \mathrm{Th}$ is a weak $\alpha$-particle emitter.

- Demonstrate the active neutron assay of ${ }^{232}$ Th with a neutron generator or high-energy isotopic source toward the goal of quantitative assay of ${ }^{232} \mathrm{Th}$ total mass.

- Evaluate the most appropriate fast neutron energy interrogation sources for the mass assay of ${ }^{232} \mathrm{Th} .{ }^{232} \mathrm{Th}$ is fissionable; therefore, thermal neutrons are not suitable for the assay of ${ }^{232} \mathrm{Th} .{ }^{232} \mathrm{Th}$ has a low IF cross-section at low energies. Only fast neutrons with energies above 1.5 MeV can yield a practically significant amount of IF on ${ }^{232} \mathrm{Th}$, although suppressed by a factor of three to four compared to ${ }^{238} \mathrm{U}$. Consider neutron sources $>1.5 \mathrm{MeV}$ for ${ }^{232} \mathrm{Th}$ active assay. However, ${ }^{238} \mathrm{U}$ has a similar fission threshold; therefore, methods to distinguish between these two isotopes must be developed.

- Evaluate neutron NDA techniques used for ${ }^{238} \mathrm{U}$ for their applicability to the assay of ${ }^{232} \mathrm{Th}$.

- Develop a concept for active neutron interrogation and perform mass assay of ${ }^{233} \mathrm{U}$ in the presence of other isotopes of uranium including fissile ${ }^{235} \mathrm{U}$ and fertile ${ }^{238} \mathrm{U}$, or fissile ${ }^{239} \mathrm{Pu}$, or all these isotopes.

- Evaluate the signature contributions from $(a, n)$ neutrons vs. correlated neutrons from fission.

- Evaluate the use of multiple interrogation sources (with different neutron energies) or the dual-energy interrogation method for applicability to the assay of ${ }^{232} \mathrm{Th}$ in the presence of ${ }^{238} \mathrm{U}$, as well as the assay of ${ }^{233} \mathrm{U}$ in the presence of ${ }^{235} \mathrm{U}$.

- Use at least two or three different neutron interrogation spectrum energies for composite materials. Possible neutron source options to be evaluated include AmLi, ${ }^{252} \mathrm{Cf}$, and neutron generators.

- Develop active neutron interrogation techniques for ${ }^{233} \mathrm{U}$ that are distinct from ${ }^{235} \mathrm{U}$-induced fission. This could potentially be achieved by algorithms including timing windows or exploration of a range of interrogating neutron spectrum energies.

- Explore a range of neutron interrogation sources for a range of neutron interrogation energies for the assay of ${ }^{233} \mathrm{U}$ in the presence of other fissile nuclides.

- Investigate the effect within the thorium MSR fuel cycle of performing neutron measurements of isotopes of safeguards interest in the presence of protactinium and its fissile and fertile isotopes (e.g., ${ }^{231,232} \mathrm{~Pa}$ ). For example, when circulating in the salt or when it is present in the decay tank, 
Concept Development and Laboratory Demonstration for Neutron NDA of ${ }^{232}$ Th and ${ }^{233} \mathrm{U}$

fissile ${ }^{232} \mathrm{~Pa}$ could be regarded as an "interference" to active neutron measurements. When using a thermal neutron interrogation source, only fissile isotopes have the potential to interfere. When using fast neutron interrogation sources (e.g. ${ }^{252} \mathrm{Cf}$ ), all actinide isotopes with a fast neutron cross-section have the potential to interfere.

\section{Concept Development for Gamma NDA of ${ }^{232}$ Th and ${ }^{233} \mathrm{U}$}

- Address the significant safeguards measurement challenge of promptly identifying ${ }^{233} \mathrm{U}$, especially if shielded.

- Address the safeguards measurement challenge of verifying ${ }^{232} U$ concentrations in ${ }^{233} U$. Develop methods to assay ${ }^{233} \mathrm{U}$ in the presence of ${ }^{232} \mathrm{U}$. The authors are not aware of any COTS analysis software for this application. We recommend that the FRAM code (or other gamma ray isotopic analysis codes) be modified for use with high-resolution gamma detectors to incorporate this analysis.

- Assay the isotopic composition of bulk ${ }^{233} \mathrm{U}$-bearing material in the presence of ${ }^{232} \mathrm{U}$ and in shielded configurations. Identify the dynamic range of feasibility of different gamma-based NDA methods considering different ${ }^{232} \mathrm{U} /{ }^{233} \mathrm{U}$ ratios and in the presence of shielding with different shielding configurations.

- Distinguish between ${ }^{232} \mathrm{Th}$ material and other materials bearing ${ }^{232} \mathrm{U}$. Due to the short half-life of ${ }^{232} \mathrm{U}$, its progeny grow in quickly and dominate the gamma ray signature of thorium fuel cycle materials. Once the ${ }^{232} \mathrm{U}$ progeny grows in, the ${ }^{232} \mathrm{U}$ gamma ray signature is similar to the ${ }^{232} \mathrm{Th}$ signature. Furthermore, the intense high-energy gamma rays of the ${ }^{232} \mathrm{U}$ daughters lead to a significant Compton continuum, especially in shielded configurations.

- Explore methods to distinguish between ${ }^{232} \mathrm{Th}$ and ${ }^{232} \mathrm{U}$ in freshly separated thorium, which is a significant challenge.

- Evaluate the use of high-resolution gamma detectors, including the DNN R\&D Safeguards Program-developed high-resolution microcalorimeter, for freshly separated thorium, considering the need to distinguish weak contributions from ${ }^{232} \mathrm{U}$ direct gamma rays.

- Utilize the ${ }^{228} \mathrm{Ac}$ gamma ray signatures in analysis method development. The ${ }^{232} \mathrm{U}$ decay chain does not include ${ }^{228} \mathrm{Ac}$, which contributes several significant gamma rays: $338 \mathrm{keV}, 911 \mathrm{keV}$, and $969 \mathrm{keV}$. Using the $911 / 969 \mathrm{keV}$ complex to distinguish between ${ }^{232} \mathrm{U}$-containing items and ${ }^{232} \mathrm{Th}$ shows potential.

- Develop concepts for inverse analysis to determine the amount of shielding present. Unlike other uranium-bearing materials, ${ }^{233} \mathrm{U}$-bearing items can have a large gamma ray dose, which is mostly due to the ingrowth of ${ }^{208} \mathrm{Tl}$ ( $2614 \mathrm{keV}$ gamma ray) in the ${ }^{232} \mathrm{U}$ decay chain. The high radiation drives shielded requirements, and the impact of shielding on the assay of ${ }^{233} \mathrm{U}$ needs to be quantified. 
Technology Development for Neutron NDA

- Develop detectors and electronics components for use in high-radiation environments.

Technology Development for Gamma NDA

- Develop detectors and electronics components for use in high-radiation environments.

Develop Safeguards Concepts and Approaches, and Policy Specific to ${ }^{232} \mathrm{Th} /{ }^{233} \mathrm{U}$

- Distinguish an analogy of LEU and HEU for ${ }^{233} \mathrm{U}$-bearing items.

- Develop protactinium safeguards concepts and approaches, including monitoring, as a precursor to pure ${ }^{233} \mathrm{U}$.

- Evaluate the potential benefits of monitoring thorium source material earlier in the fuel cycle for continuity of knowledge throughout other fuel cycle stages.

- Understand dose rates and shielding considerations as they relate to inspector access and measurement access (i.e., remote and unattended monitoring) for the development of safeguards concepts and approaches for monitoring thorium fuel cycle items.

\subsection{R\&D Needs-Higher TRL (5+) Technologies and Application- Specific Demonstration, and Safeguards Implementation}

The following recommendations are categorized as higher TRL (5+), where technology development activities are needed for application of existing safeguards technologies (both instruments and methods) to thorium fuel cycles. Further development of existing NDA systems and safeguards technologies for the assay of thorium fuel cycle items is needed. These activities include further development of instrumentation (e.g., detectors and associated instrumentation), analysis software, and embedded analysis algorithms and interpretation. Other development activities include testing and evaluation of existing technologies and methods for the assay of thorium fuel cycle material and items. This higher TRL also applies to understanding best practices for implementing existing safeguards activities within thorium fuel cycles.

\section{Materials and Infrastructure for Experimental Validation}

- Design and build a mock-up fuel assembly with thorium and uranium pins for experimental measurement campaigns and laboratory testing of developed NDA systems. This is important to testing and verification of fresh fuel attributes.

- A mock-up fuel assembly represents the highest fidelity for testing and verification of fresh fuel attributes while also representing some of the most important spent fuel characteristics (e.g., multiplication, source distribution, self-shielding). 


\section{Materials and Infrastructure for Experimental Validation}

- Stage composite items or materials (e.g., ${ }^{233} \mathrm{U}+\mathrm{Pu}, \mathrm{Th}+\mathrm{Pu}, \mathrm{Th}+\mathrm{U}$ ) for experimental measurement campaigns and laboratory testing of stand-alone detectors and developed NDA systems.

- First steps could be to measure groupings of individual sealed sources of these isotopes.

- Bulk materials are required for neutron NDA measurements.

- Use high-fidelity simulations to prescreen the significance of certain source arrangements and properties prior to procurement, and for experimental campaign design, in case of resourceintensive scenarios.

- Use high-fidelity simulations to benchmark any measurements, and for extrapolation into realistic or probable scenarios that are not achievable under laboratory conditions.

\section{Technology Development for Neutron NDA}

- Adapt INCC analysis software for the automatic evaluation of neutron-counting rates from thorium fuel cycle isotopes.

- Understand and overcome the practical constraints of applying existing neutron-counting systems to the mass assay of ${ }^{233} \mathrm{U}$ in the presence of ${ }^{232} \mathrm{U}$ gamma background (i.e., ${ }^{208} \mathrm{Tl}$ ). Understand the impact of neutron NDA system settings (i.e., high-voltage reduction needed to compensate for any background gamma dose), which, for example, might lead to longer counting times.

\section{Technology Development for Gamma NDA}

- Evaluate the concept of operations to move from high-resolution to low-resolution detectors in some cases. High-resolution detectors do not necessarily have the same automated analysis as FLIR's identiFINDER. Because the identiFINDER is currently the only gamma spectrometer in the IAEA's CA Toolkit, the concept of operations needs to be identified and the software adapted accordingly.

- Evaluate the performance of automated RIIDs, such as FLIR's identiFINDER HM-5, for isotopic identification using the $911 / 969 \mathrm{keV}$ complex to improve current analysis.

- Adapt isotopic composition analysis software and supporting nuclear data/analysis libraries for automatic evaluation of gamma ray spectra from thorium fuel cycle isotopes.

- Consider modifications to equipment and conduct of operations needed for CA Toolkits and MCIKs. For example, FLIR's identiFINDER is not capable of identifying shielded ${ }^{233} \mathrm{U}$ using current identiFINDER HM-5 analysis. 
- Evaluate effects of increasing inspection assay times (for the assay of low-intensity gamma rays associated with ${ }^{233} \mathrm{U}$ ) to improve sensitivity and optimize use of inspection resources. Evaluate concept of operations to move from low- and medium-resolution detectors to high-resolution detectors for thorium fuel cycle applications.

\section{Feasibility Study for DA}

- Perform a feasibility study to ascertain whether the current distribution system of cotton swipes by the IAEA to NWAL is fast enough to allow accurate ${ }^{233} \mathrm{~Pa}$ measurements before significant radioactive decay has occurred. This feasibility study could be performed using a cotton swipe doped with ${ }^{233} \mathrm{~Pa}$.

\section{Perform Nuclear Data Scoping and International Target Value (ITV) Definitions (Nuclear Data for Thorium} Fuel Cycle Safeguards)

- Undertake scoping effort on uncertainty implications of nuclear data for safeguards measurements.

- Begin compiling "virtual ITVs for thorium fuel cycles," which in turn could help us prioritize midto long-term R\&D efforts.

- Explore signatures feasibility and the significance of the nuclear data input.

- Perform sensitivity analysis and evaluate whether the uncertainty in the nuclear data is sufficient once methods are demonstrated or assumed feasible.

- Revisit prior studies citing the need to improve the relative uncertainties in the gamma ray absolute emission probabilities for ${ }^{233} \mathrm{U}[90]$.

\section{References}

\section{Executive Summary and Introduction References}

1. International Atomic Energy Agency, INFCIRC/140, "Treaty on the Non-Proliferation of Nuclear Weapons," April 22, 1970.

2. IAEA Department of Safeguards, Research and Development Plan: Enhancing Capabilities for Nuclear Verification, STR-385, Vienna, Austria: International Atomic Energy Agency, January 2018. https://www.iaea.org/sites/default/files/19/07/cn-267-symposium-report.pdf.

3. International Atomic Energy Agency, Report: Symposium on International Safeguards: Building Future Safeguards Capabilities, 5-8 November 2018, no. STR-392, Vienna, Austria: International Atomic Energy Agency, July 2019.

4. L. G. Worrall, et al., "Safeguards Challenges and Safeguards Technology Needs Assessment for Leading Thorium Fuel Cycles," presented at the Symposium on International Safeguards: Building 
Future Safeguards Capabilities, International Atomic Energy Agency, Vienna, Austria, November 2018.

5. E. Uribe, "Protactinium Presents a Challenge for Safeguarding Thorium Reactors," presented at the Symposium on International Safeguards: Building Future Safeguards Capabilities, International Atomic Energy Agency, Vienna, Austria, November 2018.

6. International Atomic Energy Agency, INFCIRC/153 (corrected), "The Structure and Content of Agreements Between the Agency and States Required in Connection with the Treaty on the NonProliferation of Nuclear Weapons," 1972.

https://www.iaea.org/sites/default/files/publications/documents/infcircs/1972/infcirc153.pdf.

7. IAEA Nuclear Energy Series, International Safeguards in Nuclear Facility Design and Construction, no. NP-T-2.8, Vienna, Austria: International Atomic Energy Agency, 2013.

https://www.iaea.org/publications/10361/international-safeguards-in-nuclear-facility-designand-construction.

8. International Atomic Energy Agency, IAEA Safeguards Glossary: 2001 Edition, International Nuclear Verification Series (no. 3), Vienna, Austria: International Atomic Energy Agency, 2002.

https://www.iaea.org/sites/default/files/iaea safeguards glossary.pdf.

9. W. A. Wieselquist, R. A. Lefebvre, and M. A. Jessee, Eds., SCALE Code System, Version 6.2.4, ORNL/TM-2005/39, Oak Ridge, TN: Oak Ridge National Laboratory, 2020. Available from Radiation Safety Information Computational Center as CCC-834.

10. D. Wiarda, M. E. Dunn, N. M. Greene, C. Celik, and L. M. Petrie, AMPX-6: A Modular Code System for Processing ENDF/B, ORNL/TM-2016/43, Oak Ridge, TN: Oak Ridge National Laboratory, 2016. Available from Radiation Safety Information Computational Center as CCC-834.

11. V. Fournier, "What's in An Inspector's Luggage? A Review of Safeguards Equipment," IAEA Bulletin, June 2016, 12-15.

12. A. Krichinsky, J. Giaquinto, and D. Canaan, "Workshop on Preserving High Purity Uranium-233," Proceedings of the Tenth International Conference on Methods and Applications of Radioanalytical Chemistry (MARC X), Kailua-Kona, Hawaii, April 12-17, 2015.

13. A. Krichinsky, C. Bayne, D. Bostick, J. Giaquinto, S. Goldberg, M. Humphrey, I. Hutcheon, and T. Sobolev, "The Next Generation Safeguards Initiative's Ultra-High-Purity Uranium-233 Preservation Effort," Proceedings of the Institute of Nuclear Materials Management (INMM) Annual Meeting, July 2012.

14. Nuclear Energy Agency, Uranium 2018: Resources, Production and Demand, NEA\#7413, December 13, 2018.

15. M. B. Schaffer, "Abundant Thorium as an Alternative Nuclear Fuel: Important Waste Disposal and Weapon Proliferation Advantages," Energy Policy 60 (2013): 4-12.

16. B. Ade, A. Worrall, J. Powers, S. Bowman, G. Flanagan, and J. Gehin, Safety and Regulatory Issues of the Thorium Fuel Cycle, NUREG/CR-7176, ORNL/TM-2013/543, Rockville, MD: Nuclear Regulatory Commission, February 2014.

17. International Atomic Energy Agency, Thorium Fuel Cycle-Potential Benefits and Challenges, IAEATECDOC-1450, May 2005. 
18. World Nuclear Association, Thorium, updated November 2020. https://www.worldnuclear.org/information-library/current-and-future-generation/thorium.aspx

19. D. Das and S.R. Bharadwaj (Eds.), Thoria-based Nuclear Fuels: Thermophysical and Thermodynamic Properties, Fabrication, Reprocessing, and Waste Management. Netherlands: Springer London, 2013. https://www.springer.com/us/book/9781447155881

\section{Section 1 References}

20. R. Wigeland, et al., Nuclear Fuel Cycle Evaluation and Screening-Final Report, INL/EXT-14-31465, October 2014.

21. P. K. Vijayan, V. Shivakumar, S. Basu, and R. K. Sinha, "Role of Thorium in the Indian Nuclear Power Programme,” Progress in Nuclear Energy 101 (2017): 43-52.

22. S. C. Chetal, V. Balasubramaniyan, P. Chellapandi, P. Mohanakrishnan, P. Puthiyavinayagam, C. P. Pillai, S. Raghupathy, T. K. Shanmugham, and C. S. Pillai, "The Design of the Prototype Fast Breeder Reactor," Nuclear Engineering and Design 236 (2006): 852-860.

23. K. Anantharaman, V. Shivakumar, and D. Saha, "Utilisation of Thorium in Reactors," Journal of Nuclear Materials 383 (2008): 119-121.

24. M. Allibert, E. Merle-Lucotte, V. Ghetta, T. Ault, S. Krahn, R. Wymer, A. Croff, P. Baron, N. Chauvin, R. Eschbach, et al. Introduction of Thorium in the Nuclear Fuel Cycle. Short- to long-term considerations, Paris, France: Organisation for Economic Co-Operation and Development, 2015.

25. K. I. Björk, V. Fhager, and C. Demazière, "Comparison of Thorium-Based Fuels with Different Fissile Components in Existing Boiling Water Reactors," Progress in Nuclear Energy 53 (2011): 618-625.

26. Electric Power Research Institute. Program on Technology Innovation: Technology Assessment of a Molten Salt Reactor Design, The Liquid Fluoride Thorium Reactor (LFTR), Technical Report, October 2015.

27. C. Y. Zou, C. Z. Cai, C. G. Yu, J. H. Wu, and J. G. Chen. Transition to thorium fuel cycle for TMSR. Nuclear Engineering and Design 330 (2018): 420-428.

28. Jorgensen, Lars. ThorCon reactor. In Molten Salt Reactors and Thorium Energy, 57-564pp. 5published by Woodhead Publishing, 2017.

\section{Section 2 References}

29. B. T. Rearden and M. A. Jessee, SCALE Code System version 6.2.3, ORNL/TM-2005/39, Oak Ridge, TN: Oak Ridge National Laboratory, 2018.

30. M. Todosow, A. Galperin, S. Herring, M. Kazimi, T. Downar, and A. Morozov, "Use of Thorium in Light Water Reactors," Nuclear Technology 151, no. 2 (2017): 168-176, published online April 10, 2017.

31. W. B. Arthur, Uranium Production in Thorium/Denatured Uranium Fueled PWRs, Technical Report K/OP-241, Oak Ridge, TN: Oak Ridge Gaseous Diffusion Plant, 1977.

32. M. S. Kazimi, K. R. Czerwinski, M. J. Driscoll, P. Heizlar, and J. E. Meyer, On the Use of Thorium in Light Water Reactors, MIT-NFC-TR-016, April 1999. 
33. International Atomic Energy Agency, Nuclear Power Reactors in the World, Reference Data Series no. 2, Vienna, Austria: International Atomic Energy Agency, 2020.

34. R. K. Sinha, P. Chellapandi, G. Srinivasan, I. V. Dulera, P. K. Vijayan, and S. K. Chande, "Generation IV Concepts: India," in Handbook of Generation IV Nuclear Reactors, published by Sawton and Cambridge, UK: Woodhead Publishing, 413-452.

35. World Nuclear Association, "Nuclear Power in India," updated January 2021, https://www.worldnuclear.org/information-library/country-profiles/countries-g-n/india.aspx.

36. S. S. Bajaj and A. R. Gore, “The Indian PHWR,” Nuclear Engineering and Design 236 (2006): 701-722.

37. University Network of Excellence in Nuclear Engineering. The Essential CANDU Wm. J. Garland, Editor-in-Chief, Professor Emeritus, McMaster University, Hamilton, Ontario. https://www.unene.ca/education/candu-textbook.

38. R. K. Sinha and A. Kakodkar, "Design and development of the AHWR-The Indian Thorium Fuelled Innovative Nuclear Reactor," Nuclear Engineering and Design 236 (2006): 683-700.

39. S. F. Ashley, B. A. Lindley, G. T. Parks, W. J. Nutall, R. Gregg, K. W. Hesketh, U. Kannan, P. D. Krishnani, B. Singh, A. Thakur, M. Cowper, and A. Talamo, "Fuel Cycle Modelling of Open Cycle Thorium-Fuelled Nuclear Energy Systems," Annals of Nuclear Energy 69 (2014): 314-330.

40. International Atomic Energy Agency, Fast Reactor Database, 2006 Update, IAEA TECDOC-1531, Vienna, Austria: International Atomic Energy Agency, 2006. https://wwwpub.iaea.org/MTCD/Publications/PDF/te_1531_web.pdf.

41. S. M. Lee, S. Govindarajan, R. Indira, T. M. John, P. Mohanakrishnan, R. Shankar Singh, and S. B. Bhoje, Conceptual design of PFBR Core, Kalpakkam, Tamil Nadu, India: Indira Gandhi Centre for Atomic Research, 1996.

42. P. Puthiyavinayagam, P. Selvaraj, V. Balasubramaniyan, S. Raghupathy, K. Velusamy, K. Devan, B. K. Nashine, G. P. Kumar, K. V. Suresh Kumar, S. Varatharajan, P. Mohanakrishnan, G. Srinivasan, and A. K. Bhaduri, "Development of Fast Breeder Reactor Technology in India," Progress in Nuclear Engineering 101 (2017): 19-42.

43. P. Vicente Valdez Jr., B. R. Betzler, W. A. Wieselquist, and M. Fratoni, Modeling Molten Salt Reactor Fission Product Removal with SCALE, ORNL/TM-2019/1418, February 2020.

44. B. R. Betzler, K. B. Bekar, W. Wieselquist, S. W. Hart, and S. G. Stimpson, "Molten Salt Reactor Fuel Depletion Tools in SCALE." In Proceedings of Global/Top Fuel 2019, Seattle, Washington, , September 22-26, 2019.

45. R. C. Robertson, Conceptual Design Study of a Single-Fluid Molten-Salt Breeder Reactor. Technical Report ORNL-4541, Oak Ridge, TN: Oak Ridge National Laboratory, 1971.

46. D. J. Mitchell, L. T. Harding, G. G. Thoreson, and S. M. Horne, GADRAS Detector Response Function, SAND2014-19465, Albuquerque, NM: Sandia National Laboratories, 2014.

47. J. E. Rushton, et al., Technical Handbook of ${ }^{233} U$ Material Properties, Processing, and Handling Guidelines, ORNL/TM-13600, Oak Ridge, TN: Oak Ridge National Laboratory, March 1999.

48. Swift, A., Hogue, K. K., Folk, T., and Cooley, J. Diversion Path Analysis of Thorium Molten Salt Reactors (Y/PM-20-038). Oak Ridge, TN: Y-12 National Security Complex, 2020. 


\section{Section 3 References}

49. ENDF/B-VIII.0 cross-section database, https://www.oecd-nea.org/janis/.

50. JENDL cross-section database, https://wwwndc.jaea.go.jp/jendl/j40/j40.html.

51. N. E. Holden and D. C. Hoffman, "Spontaneous Fission Half-Lives for Ground-State Nuclides," Pure and Applied Chemistry 72, no.8 (2000): 1525-1562.

52. D. Reilly, N. Ensslin, H. Smith Jr., S. Krelner, Passive Nondestructive Assay of Nuclear Materials, NUREG/CR-5550, Nuclear Regulatory Commission; ISBN: 0-16-032724-5.

53. National Physical Laboratory, Good Practice Guide No. 34, Issue 2, Radiometric Non-Destructive Assay, National Physical Laboratory, Teddington, United Kingdom, 2012.

54. IAEA Safety Standards, Safety of Radiation Generators and Sealed Radioactive Sources, Safety Guide No. RS-G-1.10, IAEA, Vienna, 2006.

55. M. A. Root, H. O. Menlove, R. C. Lanza, C. D. Rael, K. A. Miller, and J. B. Marlow, "Technical Basis for the Use of a Correlated Neutron Source in the Uranium Neutron Coincidence Collar," Nuclear Technology 197, no. 2 (2017): 180-190; DOI: 10.13182/NT16-50.

56. M. A. Root, H. O. Menlove, R. C. Lanza, C. D. Rael, K. A. Miller, J. B. Marlow, and J. G. Wendelberger "Using the Time-Correlated Induced Fission Method to Simultaneously Measure the ${ }^{235} \mathrm{U}$ Content and the Burnable Poison Content in LWR Fuel Assemblies," Nuclear Technology 203, no. 1 (2018): 34-47; DOI: $10.1080 / 00295450.2018 .1429112$.

57. J. Williams, "Development of a Neutron Generator Facility at Simon Fraser University," master's thesis, Simon Fraser University, 2013.

58. H. O. Menlove, S. H. Menlove, and S. J. Tobin, "Fissile and Fertile Nuclear Material Measurements Using a New Differential Die-Away Self-Interrogation Technique," Nuclear Instruments and Methods in Physics Research A 602 (2009): 588-593.

59. H. O. Menlove, S. H. Menlove, and S. J. Tobin, "Verification of Plutonium Content in Spent Fuel Assemblies Using Neutron Self-Interrogation," in the Proceedings of the $50^{\text {th }}$ Annual Meeting of the Institute of Nuclear Materials Management, Tucson, Arizona, July 12-16 2009.

60. A. C. Kaplan, V. Henzl, A. P. Belian, H. O. Menlove, M. T. Swinhoe, M. Flaska, and S. A. Pozzi, "Determination of Spent Nuclear Fuel Assembly Multiplication with the Differential Die-Away SelfInterrogation Instrument," Nuclear Instruments and Methods A 757, no. 1 (2014): 20-27.

61. H. O. Menlove, M. T. Swinhoe, and K. A. Miller. "A More Accurate and Penetrating Method to Measure the Enrichment and Mass of $\mathrm{UF}_{6}$ in Storage Cylinders Using Passive Neutron SelfInterrogation," In Proceedings of the $51^{\text {st }}$ INMM Annual Meeting, Baltimore, Maryland, July 11-15, 2010.

62. L. E. Smith, K. A. Miller, B. S. McDonald, J. B. Webster, M. A. Zalavadia, J. R. Garner, S. L. Stewart, S. J. Branney, L. C. Todd, N. S. Deshmukh, H. A. Nordquist, J. A. Kulisek, and M. T. Swinhoe, "An Unattended Verification Station for $\mathrm{UF}_{6}$ Cylinders: Field Trial Findings," Nuclear Instruments and Methods in Physics Research A: Accelerators, Spectrometers, Detectors and Associated Equipment 874 (2017): 127-136. 
63. H. O. Menlove, S. H. Menlove, and C. D. Rael. "The Development of a New, Neutron, Time Correlated, Interrogation Method for Measurement of ${ }^{235} \mathrm{U}$ Content in LWR Fuel Assemblies." Nuclear Instruments and Methods in Physics Research Section A: Accelerators, Spectrometers, Detectors and Associated Equipment 701 (2013): 72-79.

64. H.O. Menlove, Description and Operation Manual for the Active Well Coincidence Counter, LA-7823-M Manual (ISPO-66), Los Alamos, NM: Los Alamos Scientific Laboratory, , May 1979.

65. M. A. Humphrey, S. J. Tobin, and K. D. Veal. "The Next Generation Safeguards Initiative's Spent Fuel Nondestructive Assay Project," Journal of Nuclear Materials Management 40, no. 3 (2012): 1-6.

66. V. Henzl, Evaluation of Differential Die-Away Technique Potential in Context of Non-Destructive Assay of Spent Nuclear Fuel, LA-UR-29224, Los Alamos, NM: Los Alamos National Laboratory, 2014.

67. A. L. Lousteau, R. D. McElroy, S. Croft, and J. P. Hayward, "Determining 235 U Enrichment in Bulk Uranium Items Using Dual-Energy Interrogation with Delayed Neutron Measurement," Nuclear Instruments and Methods in Physics Research Section A: Accelerators, Spectrometers, Detectors and Associated Equipment, 904 (2018): 74-80; ISSN 0168-9002, https://doi.org/10.1016/j.nima.2018.07.030, http://www.sciencedirect.com/science/article/pii/S0168900218308659.

68. H. O. Menlove, C. D. Rael, K. E. Kroncke, and K. J. DeAguero, Manual for the Epithermal Neutron Multiplicity Detector (ENMC) for Measurement of Impure MOX and Plutonium Samples, Technical Report LA-14088, Los Alamos, NM: Los Alamos National Laboratory, 2004.

69. D. M. Cifarelli and W. Hage, "Models for a Three Parameter Analysis of Neutron Signal Correlation Measurements for Fissile Material Assay," Nuclear Instruments and Methods in Physics Research Section A: Accelerators, Spectrometers, Detectors and Associated Equipment A251 (1986): 550.

70. INCC Software Users Manual, Technical Report LA-UR-10-6277, Los Alamos, NM: Los Alamos National Laboratory, March 2009.

71. S. Croft, A. Favalli, M. T. Swinhoe, and C. D. Rael, "State of the Art Monte Carlo Modeling of Active Collar Measurements and Comparison with Experiment," in Proceedings of the 52nd Annual INMM Meeting, Palm Desert, CA, July 17-21, 2011.

72. R. Boll, K. Murphy, G. Groover, and S. Mirzadeh, "Production of Ac-225 for Alpha Particle Radioimmunotherapy," Applied Radiation and Isotopes 62, no. 5 (May 2008): 667-679.

73. J. Kang and F. N. von Hippel, "U-232 and the Proliferation Resistance of U-233 in Spent Fuel," Science and Global Security 9, no. 1 (2001): 1-32; DOI:10.1080/08929880108426485.

74. Decay Chain of Thorium. https://en.wikipedia.org/wiki/Decay chain

75. V. Henzl, R. E. Lakis, K. E. Koehler, and D. J. Desimone, "Safeguards Technology Development Complementary Access Tools Project (Final Report)“, Technical Report LA-CP-19-20839 (OUO only), Los Alamos, NM: Los Alamos National Laboratory.

76. D. T. Vo and T. E. Sampson, FRAM, Version 5 User's Manual, Technical Report LA-UR-11-03005, Los Alamos, NM: Los Alamos National Laboratory.

77. J. K. Mattingly, D. J. Mitchell, and C. L. Rhykerd Jr., Benchmarks for GADRAS Performance Validation, SAND2009-6550, Albuquerque, NM: Sandia National Laboratories, January 2009. 
78. National Nuclear Laboratory, Comparison of thorium and uranium fuel cycles, NNL (11) 11593 Issue 5, March 2012.

79. S. F. Ashley, G. T. Parks, W. J. Nuttall, C. Boxall, R. W. Grimes, “Thorium Fuel Has Risks.” Nature 492 (2012), 31-33.

80. R. M. Essex, J. L. Mann, R. W. Williams, W. S. Kinman, A. Hubert, M. E. Bennett, and A. Gourgiotis, "A New Thorium-229 Reference Material.” Applied Radiation and Isotopes 134 (2018): 23-31.

\section{Section 4 References}

81. L. Rockwood, "Legal Framework for IAEA Safeguards," International Atomic Energy Agency, 2013, https://www.iaea.org/publications/10388/legal-framework-for-iaea-safeguards.

82. Statute of the IAEA, https://www.iaea.org/sites/default/files/statute.pdf.

83. INFCIRC/540/Corr., "Model Protocol Additional to the Agreement(s) Between State(s) and the International Atomic Energy Agency for the Application of Safeguards," https://www.iaea.org/sites/default/files/infcirc540c.pdf

84. INFCIRC/66/Rev.2, "The Agency's Safeguards System," https://www.iaea.org/publications/documents/infcircs/agencys-safeguards-system-1965.

85. "2018 IAEA Annual Report: Additional Annex Information," GC(63)/5, https://www.iaea.org/publications/reports

86. Complementary Access Working Group Phase 1, Working Group on Complementary Access Tools, Phase 1 Final Report, March 2013 (OUO only).

87. Complementary Access Working Group Phase 2, Working Group on Complementary Access Tools, Phase 2 Final Report, October 2013 (OUO only).

88. Complementary Access Working Group Phase 3, Identification of Chemicals of Interest in the Nuclear Fuel Cycle for Safeguards through Laboratory and Field Testing: Complementary Access Working Group Phase 3 Report, INL/EXT-14-33072, Revision 1, November 2014 (OUO only).

89. International Atomic Energy Agency, Development and Implementation Support for Nuclear Verification 2020-2021, STR-393, Vienna, Austria: International Atomic Energy Agency, January 2020.

\section{Section 5 References}

90. L. G. Worrall, R. D. McElroy, A. M. Krichinsky, S. L. Cleveland, and S. Croft, "Uranium-233 Signatures," in Proceedings of the INMM 55 th Annual Meeting, Atlanta, Georgia, July 20-24, 2014. 


\section{Bibliography: General Safeguards}

International Atomic Energy Agency. IAEA Annual Report 2018: Additional Annex Information (GC(63)/5/Annex). Vienna, Austria: International Atomic Energy Agency, 2018.

International Atomic Energy Agency. IAEA Safeguards Glossary. Vienna, Austria: International Atomic Energy Agency, 2001.

International Atomic Energy Agency. International Target Values 2010 for Measurement Uncertainties in Safeguarding Nuclear Materials (STR-368). Vienna, Austria: International Atomic Energy Agency, 2010.

International Atomic Energy Agency. "List of Nuclear Fuel Cycle Facilities.” [Online]. Accessed January 2020. Available: https://infcis.iaea.org/NFCIS/Facilities

International Atomic Energy Agency, Model Protocol Additional to the Agreement(s) between State(s) and the International Atomic Energy Agency for the Application of Safeguards (INFCIRC/540). 1997.

International Atomic Energy Agency. Nuclear Material Accounting Handbook (Services Series 15). Vienna, Austria: International Atomic Energy Agency, 2008.

International Atomic Energy Agency. The Safeguards Implementation Report for 2019 (GOV/2020/9). Vienna, Austria: International Atomic Energy Agency, 2020.

International Atomic Energy Agency. The Structure and Content of Agreements between the Agency and States Required in Connection with the Treaty on the Non-proliferation of Nuclear Weapons (INFCIRC/153 (Corrected)). Vienna, Austria: International Atomic Energy Agency, 1972.

Rockwood, L. Legal Framework for IAEA Safeguards. Vienna, Austria: International Atomic Energy Agency, Vienna, 2013.

Sager, H., Reed, J., and Heine, P. IAEA Safeguards Implementation Report Visualizations: State Visualizations Atlas. Lemont, IL: Argonne National Laboratory, 2019. 


\section{Bibliography: Safeguards for Thorium Fuel Cycles}

Ashley, S. F., Parks, G. T., Nuttall, W. J., Boxall, C., and Grimes, R. W. "Thorium Fuel Has Risks.” Nature 492 (2012): 31-33.

Bathke, C., Sleaford, B., Ebbinghaus, B., Collins, B., Wallace, R., Hase, K., Bradley, K., Prichard, A., and Smith, B. "An Assessment of the Attractiveness of Material Associated with Thorium/Uranium and Uranium Closed Fuel Cycles from a Safeguards Perspective." Presented at the INMM 51st Annual Meeting, Baltimore, Maryland, July 11-15, 2010.

Boyer, B. D., Beddingfield, D. H., Durst, P., and Bean, R. "Pebble Bed Modular Reactor Safeguards: Developing New Approaches and Implementing Safeguards by Design," Presented at the Pacific Northwest International Conference on Global Nuclear Security-the Decade Ahead, Portland, Oregon, April 11-16, 2010.

Bueker, H. "Safeguards-Systems for Pebble Bed Reactors." Presented at the INMM 17th Annual Meeting, Seattle, Washington, June 1976.

Chirayath, S. S., Aoyagi, R., and Sagara, H. "Feasibility Study of Nuclear Material Accounting in a Molten Salt Fast Reactor (MSFR) To Develop a Safeguards Approach." Presented at the INMM 61st Annual Meeting, Baltimore, Maryland, July 12-16, 2020.

Coble, J., Skutnik, S., Gilliam, S. N., and Cooper, M. "Review of Candidate Techniques for Material Accountancy Measurements in Electrochemical Separations Facilities." Journal of Nuclear Technology 206, no. 12 (April 2020): 1803-1826. Published online April 10, 2020.

Durst, P. C., Beddingfield, D., Boyer, B., Bean, R., Collins, M., Ehinger, M., Hanks, D., Moses, D. L., and Refalo, L. Nuclear Safeguards Considerations for the Pebble Bed Modular Reactor. Idaho Falls, ID: Idaho National Laboratory 2009.

Evans, L. G., McElroy, R. D., Krichinsky, A. M., Croft, S., and Cleveland, S.L. "Determination of Accurate Gamma-Ray Signatures for ${ }^{233}$ U." Proceedings of the ESARDA Symposium, Bruges, Belgium, May 27-30, 2013.

Fane, B. M., Murphy, C. L., and Boyer, B. D. "Thorium Based Power Systems and Relevant Safeguards Considerations." Presented at the INMM 52nd Annual Meeting, Palm Desert, CA, July 17-21, 2011.

Gangotra, S., Grover, R., and Ramakumar, K. "Analysis of Measures to Enhance Safeguards, and Proliferation Resistance in Thorium Based Fuel Fabrication Plants." Progress in Nuclear Energy 77 (2014): 20-31.

Gangotra, S., Grover, R., and Ramakumar, K. "Comparison for Thorium Fuel Cycle Facilities of Two Different Capacities for Implementation of Safeguards." Nuclear Engineering and Design 262 (2013): 535-543.

Hakkila, E., Barnes, J., Dayem, H., Dietz, R., and Shipley, J. Preliminary Concepts: Coordinated Safeguards for Materials Management in a Thorium-Uranium Fuel Reprocessing Plant (LA-7411MS). Los Alamos, NM: Los Alamos National Laboratory, 1978.

International Atomic Energy Agency, International Safeguards in the Design of Nuclear Reactors. IAEA Nuclear Energy Series. Vols. NP-T-2.9. Vienna, Austria: International Atomic Energy Agency, 2014.

International Atomic Energy Agency, Thorium Fuel Cycle-Potential Benefits and Challenges (IAEATECDOC-1450). Vienna, Austria: International Atomic Energy Agency, 2005. 
Kang, J., and von Hippel, F. N. "U-232 and the Proliferation-Resistance of U-233 in Spent Fuel." Science \& Global Security 9 (2001): 1-32.

Krahn, S. L., and Worrall, A. "The Reemergence of the Thorium Fuel Cycle: A Special Issue of Nuclear Technology," Nuclear Technology vol. 194 (2016): iii-iv.

Launiere, C., Hoyt, N., Stricker, E., and Pereira, C. "Safeguards by Design: Multimodel In-Process Monitoring of Molten Salt Composition." Presented at the INMM 61st Annual Meeting, Baltimore, Maryland, July 12-16, 2020.

Micewski, L. H., and Palmer, C. "Safeguards Considerations for Thorium Reactors." Presented at the INMM 61st Annual Meeting, Baltimore, Maryland, July 12-16, 2020.

Murphy, C., Doyle, J., Boyer, B., and Fane, B. "International Safeguards Recommendations for The Indian Advanced Heavy Water Reactor (AHWR)." Presented at the INMM 52nd Annual Meeting, Palm Desert, CA, July 17-21, 2011, 2011.

National Nuclear Laboratory, Comparison of thorium and uranium fuel cycles, NNL (11) 11593 Issue 5, March 2012.

Shoman, N., and Cipiti, B. B. Development of Continuous Processes for a Molten Salt Reactor Safeguards Model, ANS Transactions, Volume 119, Number 1, November 2018, Pages 237-240.

Swift, A., Hogue, K. K., Folk, T., and Cooley, J. Diversion Path Analysis of Thorium Molten Salt Reactors (Y/PM-20-038). Oak Ridge, TN: Y-12 National Security Complex, 2020.

Swift, A., Hogue, K. K., Folk, T., and Cooley, J. "Safeguards Technical Objectives for Thorium Molten Salt Reactor Fuel Cycles." Presented at the INMM 51st Annual Meeting, Baltimore, Maryland, July 11-15, 2010.

Todosow, M., Aronson, A. , Cheng, L.-Y, Wigeland, R., Bathke, C., Murphy, C., Boyer, B., Doyle, J., Fane, B., and Ebbinghaus, B. The Indian Advanced Heavy Water Reactor(AHWR) and Non-Proliferation Attributes (BNL-98372-2012). Upton, NY: Brookhaven National Laboratory, August 2012.

Uribe, E. "Protactinium Presents a Challenge for Safeguarding Thorium Reactors." Presented at the Symposium on International Safeguards: Building Future Safeguards Capabilities, International Atomic Energy Agency, Vienna, Austria, November 2018.

Wigeland, R., Taiwo, T., Ludewig, H., Todosow, M., Halsey, W., Gehin, J., Jubin, R., Buelt, J., Stockinger, S., Jenni, K., and Oakley, B. Nuclear Fuel Cycle Evaluation and Screening-Final Report: Fuel Cycle Research \& Development. Idaho Falls, ID: Idaho National Laboratory, 2014.

Williams, A., and Galbreth, G. "Recent Developments in the Design of a Triple Bubbler Sensor." Presented at the INMM 61st Annual Meeting, Baltimore, Maryland, July 12-16, 2020.

Worrall, L. , Worrall, A. , Flanagan, G., Croft, S. , Krichinsky, A., Pickett, C., McElroy Jr., R. D., Cleveland, S., Kovacic, D. N., Whitaker, J. M. and White-Horton, J. "Safeguards Considerations for Thorium Fuel Cycles," Journal of Nuclear Technology 194, no. 2 (May 2016): 281-293.

Worrall, L. G., McElroy, R. D., Krichinsky, A. M., Cleveland, S. L., andCroft, S. "Uranium-233 Signatures." Proceedings of the INMM 55 ${ }^{\text {th }}$ Annual Meeting, Atlanta, Georgia, July 20-24, 2014.

Worrall, L. G., et al., "Safeguards Challenges and Safeguards Technology Needs Assessment for Leading Thorium Fuel Cycles." Presented at the Symposium on International Safeguards: Building Future Safeguards Capabilities, International Atomic Energy Agency, Vienna, Austria, November 2018. 
This page intentionally left blank. 


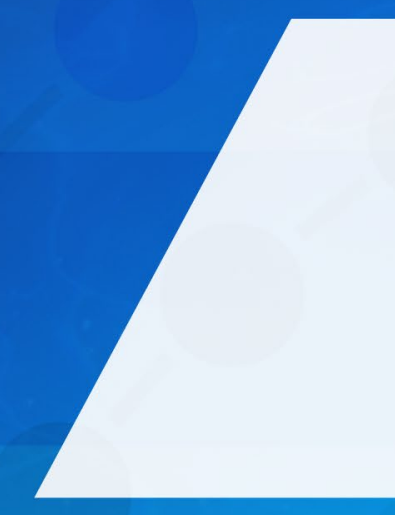

